\title{
Versuche über das Biegeverhalten von vorgespannten Platten ohne Verbund
}

\section{Working Paper}

\section{Author(s):}

Ritz, Peter; Thürlimann, Bruno; Marti, Peter

Publication date:

1975

Permanent link:

https://doi.org/10.3929/ethz-a-000396297

\section{Rights / license:}

In Copyright - Non-Commercial Use Permitted

Originally published in:

Bericht / Institut für Baustatik und Konstruktion ETH Zürich 7305(1) 
Versuche über das Biegeverhalten von vorgespannten Platten ohne Verbund

Peter Ritz

Peter Marti

Bruno Thürlimann 
(C) Birkhäuser Verlag Basel und Stuttgart, 1975

ISBN 3-7643-0796-X 


\title{
Versuche über das Biegeverhalten von vorgespannten Platten ohne Verbund
}

\author{
von \\ Peter Ritz, dipl. Ing. \\ Peter Marti, dipl. Ing. \\ Prof. Dr. Bruno Thürlimann \\ Institut für Baustatik und Konstruktion \\ Eidgenössische Technische Hochschule Zürich
}


Seite

1. EINLEITUNG

1.1 Problemstellung 1

1.2 Versuchsprogramm und Zielsetzung 1

2. VERSUCHSKOERPER 2

2.1 Beschreibung ' 2

2.2 Baustoffe 3

2.3 Rechnerische Werte 3

3. VERSUCHSDURCHFUEHRUNG

3.1 Versuchsanlage $\quad 5$

3.2 Versuchsablauf $\quad 6$

4. VERSUCHSRESULTATE 9

4.1 Platte P $1 \quad 9$

4.2 Plattenstreifen PS 1 - PS 5

$\begin{array}{lr}\text { ZUSAMMENFASSUNG } & 18\end{array}$

$\begin{array}{ll}\text { RESUME } & 20\end{array}$

$\begin{array}{ll}\text { SUMMARY } & 22\end{array}$

$\begin{array}{ll}\text { VERDANKUNGEN . } & 24\end{array}$

$\begin{array}{lr}\text { LITERATURVERZEICHNIS } & 25\end{array}$

$\begin{array}{ll}\text { BEZEICHNUNGEN } & 26\end{array}$

$\begin{array}{lllll}\text { TABELLN } 1 \text { BIS } 11 & 30\end{array}$

$\begin{array}{lllll}\text { BILER } 1 \text { BIS } 85 & 39\end{array}$

$\begin{array}{lr}\text { ANHANG A } & 104\end{array}$

$\begin{array}{lr}\text { ANHANG B } & 107\end{array}$ 


\subsection{Problemstellung}

Für die Berechnung von Stahlbetonplatten liegen heute verschiedene Methoden und Computerprogramme vor. Bei der theoretischen Untersuchung des Tragverhaltens auf Biegung von vorgespanten Platten zeigt es sich, dass ein wesentlicher Unterschied im Verhalten bei Vorspannung mit Verbund oder ohne Verbund besteht. Ist Verbund vorhanden, kann das Biegeverhalten mit den heute bekannten Methoden der Elastizitäts- und Plastizitätstheorie gut erfasst werden. Für vorgespannte Platten ohne Verbund fehlen aber allgemein gültige Berechnungsverfahren, und es sind nur wenige experimentelle Untersuchungen bekannt. Die Zugkraft im Vorspannstahl kann nicht mehr, wie üblich, aufgrund der Annahme über das Ebenbleiben des Querschnittes und mit Gleichgewichtsbetrachtungen in einem Querschnitt berechnet werden.

Das Tragverhalten von vorgespannten Platten ohne Verbund wird mit einem im Anhang $A$ dargestellten theoretischen Modell untersucht, welches eine eingehende rechnerische Behandlung ermöglicht. Durch einige gezielte Versuche sollte das theoretische Modell verifiziert und der Einfluss wesentlicher Parameter auf das Tragverhalten von vorgespanten, nichtinjizierten platten auf Biegung experimentell untersucht werden.

\subsection{Versuchsprogramm und Zielsetzung}

Das experimentelle Forschungsprogramm über das Biegeverhalten von vorgespannten, nichtinjizierten Platten ist in Tabelle 1 zusammengestellt. Es enthält die Prüfung einer umfanggelagerten Quadratplatte sowie von fünf Plattenstreifen. Bei der Quadratplatte $P 1$ wurde die ganze Belastungs-Verformungsgeschichte einer einfachen, nichtinjizierten Platte bis zum Bruch beobachtet. Die Plattenstreifen erlaubten es, den Einfluss einiger wichtiger Parameter zu studieren. Seitlich wurden die Plattenstreifen in ihrer Ebene durch eine verschieden steife feder gestützt. Damit wurde ein aus einer Platte herausgeschnittener Streifen mit frei wählbaren horizontalen Randbedingungen simuliert. Es war dadurch möglich, die Membranwirkung in den Versuch miteinzubeziehen.

Bei den Plattenstreifen PS 1 bis PS 3 wurde die Steifigkeit der horizontalen Feder variiert. Der Einfluss einer zusätzlichen schlaffen Armierung wurde im PS 4 untersucht. Schliesslich gab der Plattenstreifen PS 5 Auskunft über das Verhalten bei grossen Schlankheiten. Im speziellen wurden folgende Vorgänge und Grössen beabachtet resp. ermittelt:

- Elastisches Verhalten der Platte und Plattenstreifen

- Verhalten im Gebrauchszustand

- Ausbilden eines Zug- resp. Druckringes bei $P 1$

- Kollaps: Durch Reissen der Litzen oder Zerstören des Betons infolge grosser Stauchungen; Ausbilden von Bruchmechanismen

- Spannungszunahme im Vorspannstahl

- Verformungen

- Betonstauchungen, Rotationsfähigkeit

- Rissverhalten. 


\section{VERSUCHSKOERPER}

\subsection{Beschreibung}

Abmessungen und Armierung gehen aus den Bildern 3 bis 9 hervor. Die Plattenstärke von $18 \mathrm{~cm}$ erlaubte die Verwendung eines Betons mit üblichem Maximalkorndurchmesser und eine normale konstruktive Ausbildung. Die Platte $P 1$ und die fünf Plattenstreifen wurden in der Eidgenössischen Materialprüfungs- und Versuchsanstalt (EMPA) in Dübendorf $(Z H)$ betoniert und bis zum Versuch bei konstanter Raumtemperatur von ungefähr $20^{\circ}$ C gelagert.

Mit ihrem parabolischen Kabelverlauf und der verschwindenden Endexzentrizität werden die Versuchskörper in Anlehnung an das im Anhang A beschriebene Modell als Ausschnitte aus durchlaufenden Platten mit Spannweiten $\sqrt{2} \cdot 1$ betrachtet. In diesem Sinne können den effektiven Schlankheiten d/1 von 1/20 bzw. 1/33 (PS 5) die auf durchlaufende Platten bezogenen Werte von $1 / 28$ bzw. $1 / 47$ zugeordnet werden (vgl. Tabelle 1 ).

\section{Platte P 1}

In beiden Hauptrichtungen wurde die Platte mit neun 1/2" Litzen vorgespannt. Die Litzen hatten eine maximale mittlere Exzentrizität von $6.5 \mathrm{~cm}$, die geringste Betonüberdeckung betrug $1 \mathrm{~cm}$. An den Litzenenden wurden Presshülsen aufgestaucht, welche die Spannkraft über in die Schalung eingelassene Stahlplatten an den Beton abgaben. Auf der Festseite wurde bei acht Kabeln eine Druckmessdose zwischen Stahlplatte und Presshülse angeordnet. Auf der beweglichen Seite konnten die Litzen mit einem Gewinde bei der gewünschten Spannung fixiert werden.

Ausser einer kleinen Randarmierung auf einer Breite von $25 \mathrm{~cm}$ zur Verstärkung der Krafteinleitungsstellen wurde keine schlaffe Armierung eingelegt; insbesondere wurde auf eine Drillarmierung verzichtet.

Konische Aussparungen bei den 16 Lastpunkten ermöglichten auch bei sehr grossen Durchbiegungen eine saubere Krafteinleitung. Der kleine Querschnitt dieser Aussparungen in der Betondruckzone schwächte die Platte nur unbedeutend.

Das Vorspannen erfolgte nach 21 Tagen ( 1 Woche vor Versuchsbeginn), indem alternierend die Litzen beider Richtungen von innen bis zum Rand fortschreitend auf die erforderliche Kraft von 11.76 to $\left(0.70 \mathrm{~B}_{z}\right)$ gespannt wurden. Die Spannkraft wurde mit einem Manometer und bei acht Litzen zusätzlich mit Druckmessdosen kontrolliert. Sie betrug bei Versuchsbeginn im Mittel noch 11.25 to, was einer zentrischen Vorspannung von $14.8 \mathrm{~kg} / \mathrm{cm}^{2}$ in beiden Tragrichtungen entsprach.

\section{Plattenstreifen PS 1 bis PS 5}

Alle Plattenstreifen wurden mit einer 1/2" Litze vorgespannt. Der maximalen Exzentrizität von $7.25 \mathrm{~cm}$ entsprach die geringste Betonüberdeckung von $1 \mathrm{~cm}$.

Als Stirnseiten der Schalung dienten stählerne Endplatten, die später mit den entsprechenden Teilen der Versuchsanlage zusammengeschraubt werden konnten. Diese Endplatten übertrugen beim Versuch die Auflagerreaktionen, und auf sie stützten sich die Kabelverankerungen ab. Zur Verstärkung der Krafteinleitungszone wurden an jede End- 
platte vier Bügel angeschweisst. Bei den vier Lasteinleitungsstellen wurden unten offene Bügel aus Flachstahl angeordnet, die in der Schalung als Distanzhalter und beim Versuch zur Befestigung der Lastübertragungsplatten Verwendung fanden. Eine zusätzliche schwache Armierung aus zwei Montageeisen und unten offenen Bügeln $\emptyset 5 \mathrm{~mm}$ ergab eine weitere Verstärkung der Krafteinleitungszonen, ohne jedoch die Tragfähigkeit zu erhöhen.

Nur beim Plattenstreifen PS 4 wurde eine untere schlaffe Armierung eingelegt. Sie bestand aus drei Eisen $\square 8 \mathrm{~mm}$, welche wie die erwähnten Montageeisen nicht mit der Endplatte verschweisst wurden. Somit konnten Zugkräfte nur über Haftung beim Stoss mit den vier Bügeln an die Endplatte abgegeben werden.

Die jeweils eine woche vor dem Versuch aufgebrachte Spannkraft betrug bei Versuchsbeginn noch 11.25 to, was eine zentrische Vorspannung von $15.6 \mathrm{~kg} / \mathrm{cm}^{2}$ ergab.

\subsection{Baustoffe}

\section{Spannstahl}

Bild 1 zeigt ein aus einem Zugversuch gewonnenes Spannungs-Dehnungsdiagramm der verwendeten 1/2" Monolitzen. Da die dynamischen Festigkeitswerte von der Dehnungsgeschwindigkeit $\dot{\varepsilon}_{s}=d \varepsilon_{s} / d t$ abhängig sind, wurden die statischen werte $\left(\dot{\varepsilon}_{s}=0\right)$ der Auswertung zugrundegelegt, was dem Ablauf der Versuche entspricht. Alle Litzen waren gefettet und von einem eng anliegenden Plastikrohr $\emptyset 15 \mathrm{~mm}$ umhüllt.

\section{Schlaffe Armierung}

In Tabelle 3 sind die Festigkeitswerte des verwendeten naturharten Armierungsstahles zusammengestellt. Auch hier liegen der Auswertung die statischen Werte zugrunde.

\section{Beton}

Die Zusammensetzung des Betons war für alle Versuchskörper gleich und ist in Tabelle 2 angegeben. Die Sieblinie der Zuschlagstoffe entsprach ungefähr der EMPA-Kurve. Als Zement wurde schweizerischer Portlandzement verwendet.

Zur Ermittlung der Betonfestigkeit wurden gleichzeitig mit jedem Versuchskörper acht Prismen $12 \times 12 \times 36 \mathrm{~cm}$ hergestellt. Im Zeitpunkt des Versuches wurde jeweils an vier Prismen die Prismendruckfestigkeit und der Elastizitätsmodul und an den übrigen vier die Biegezug- und Würfeldruckfestigkeit ermittelt. Diese Werte wurden bei relativ hoher Dehnungsgeschwindigkeit gemessen und sind aus Tabelle 4 ersichtlich.

\subsection{Rechnerische Werte}

Die Ermittlung der rechnerischen Werte ist in Anhang $B$ im Detail dargelegt. Die Resultate sind in Tabelle 11 zusammengefasst.

\section{Risslasten}

Die Risslasten wurden unter Vernachlässigung des Membraneinflusses bestimmt, um dadurch allgemein geläufige Vergleichswerte zu erhalten. Die Risslast für den ersten 
Belastungszyklus wurde mit der aus den entsprechenden Biegezugversuchen ermittelten Betonzugfestigkeit berechriet, wobei dieser Wert erfahrungsgemäss eine grosse streuung aufweist. Für die Berechnung der Risslast bei den weiteren Belastungszyklen wurde der Versuchskörper als gerissen angenommen und die Betonzugfestigkeit gleich Null gesetzt.

\section{Theoretische Bruchlasten}

Die theoretischen Bruchlasten wurden unter der Annahme bestimmt, dass der Vorspannstahl als Hängewerk trägt und unmittelbar vor dem Bruch einen sinusförmigen Durchhang besitzt. Vergleichsrechnungen zeigten, dass eine parabolische oder eine trapezfärmige Annahme des Durchbiegungsverlaufes unwesentliche Abweichungen der Resultate ergeben. Dem Beton wurde keine Tragfunktion mehr zuerkannt. Um die schlaffe Armierung Fe beim Plattenstreifen PS 4 zu berücksichtigen, wurde die Annahme gemacht, dass sich das volle plastische Moment infolge $F_{e}$ ausbildet und dadurch ein zusätzlicher Biegewiderstand entsteht. Die Membranwirkung infolge $F_{e}$ wurde vernachlässigt. Die maximal mögliche Mittendurchbiegung wurde mit der aus zwei Zugversuchen ermittelten Bruchdehnung der Vorspannlitzen von 2.66\% berechnet. Beim Vergleich der theoretischen und experimentellen Bruchlasten ist zu beachten, dass sowohl die bei allen Plattenstreifen vorhandenen oberen Montageeisen als auch die untere schlaffe Armierung beim PS 4 teilweise als Hängewerk tragen konnten.

\section{Bezugswerte}

Als Vergleichswerte wurden die Bruchlasten quv unter der Annahme bestimmt, dass die Vorspannlitzen im Verbund wirken. Als weitere Werte wurden die mit den amerikanischen ACI-Empfehlungen [1] berechneten zulässigen Belastungen und Bruchlasten angegeben. 


\section{VERSUCHSDURCHFUEHRUNG}

Alle Versuche wurden auf dem Aufspannboden der Eidgenössischen Materialprüfungs- und Versuchsanstalt (EMPA) in Dübendorf $(Z H)$ durchgeführt.

\subsection{Versuchsanlage}

Für die Platte $P 1$ und die fünf Plattenstreifen mussten zwei Versuchsanlagen konstruiert werden. Dem Entwurf lag folgendes Konzept zugrunde. Einę einwandfreie Versuchsdurchführung und damit die Beobachtung des Tragverhaltens sollte auch bei grossen Verformungen möglich sein, nämlich bis zu einer der Bruchdehnung der Litzen entsprechenden Durchbiegung.

\section{Platte $P 1$}

Wie aus dem Schema des Bildes 10 und aus Bild 11 ersichtlich ist, wurde die Platte pro Seite durch drei horizontal allseitig verschiebliche Teflongleitlager gestützt. Zusammen mit einem als Auflagerschiene wirkenden ausgesteiften T-Profil ergab sich so eine horizontal allseitig bewegliche Linienkipplagerung. Die Lager stützten sich ihrerseits auf einen Unterbau aus vorfabrizierten Betonquadern ab.

Die Belastung wurde über 16 auf dem Aufspannboden verankerte Zugkalben aufgebracht, die an einem Federmanometer angeschlossen waren. Die Eckpunkte wurden mit vier Zugkolben auf konstanter Hähe gehalten.

\section{Plattenstreifen PS 1 bis PS 5}

Aus Bild 12 geht hervor, dass die vertikale Belastung analog zum versuch $p$ in vier Schnitten aufgebracht wurde. In jedem Schnitt war beidseits des Plattenstreifens je ein Zugkolben angeordnet, und die Belastung wurde über einen an seiner Unterseite mit einer Schneide versehenen Balken auf eine auf dem Versuchskörper festgeschraubte Stahlplatte übertragen. Die insgesamt acht Zugkolben waren an einem Pendelmanometer angeschlossen. Die vertikalen Auflagerkräfte wurden über die Endplatten der Plattenstreifen und seitlich geführte Teflongleitlager auf massive Stahlböcke abgegeben.

Wie in Abschnitt 1.2 erwähnt worden ist, sollten die Plattenstreifen Ausschnitte aus Platten mit frei wählbaren Randbedingungen darstellen. Die seitliche Haltung gemäss einer gewissen Federcharakteristik bedingte einerseits die Möglichkeit Horizontalkräfte auf die plattenstreifen aufzubringen, und andererseits musste die Längenänderung der plattenstreifen sehr genau gemessen werden können.

Die Horizontalkräfte wurden mit zwei nebeneinander angeordneten Druck- und Zugkolben auf die Endplatte der Versuchskörper übertragen (vgl. Bild 13). Die beiden Kolben stützten sich auf den erwähnten Stahlbock ab und wurden von einem Federmanometer (Hydro-Pacer) gesteuert. Am gegenüberliegenden Ende der Plattenstreifen übernahmen zwei gleich wie die Horizontalkolben gelagerte Pendelstangen die Horizontalreaktion (vgl. Bild 14).

Für die Versuchsdurchführung entscheidend war das genaue Erfüllen der gewünschten Randbedingungen. Für die Längenmessung der Plattenstreifen musste deshalb eine Genauigkeit von $\pm 0.01 \mathrm{~mm}$ verlangt werden. Dies entspricht bei einer Federsteifigkeit 
von z.B. $7 \cdot 10^{3}$ to/m (wie bei PS 2, PS 4, PS 5) einer Schwankung der Horizontalkraft $u m \pm 70 \mathrm{~kg}$. Um die Relativverschiebung der Endpunkte zu bestimmen, mussten Deformationen der Auflageteile aus der Messung ausgeschlossen werden. Deshalb wurde auf beiden Plattenstreifenseiten je eine Längenmesseinrichtung mit zwei Invardrähten angeordnet ( $v$ gl. Bild 14): Diese zwei Drähte waren an den Endplatten befestigt und führten über Umlenkrollen zu einem induktiven Wegaufnehmer hoher Genauigkeit. Der kürzere Draht trug das Gehäuse und den Kernkanal des Wegaufnehmers, der längere den Tauchanker. Probleme ergaben sich wegen den aus Lagerreibung der Umlenkrollen möglichen elastischen Dehnungen der Drähte. Die daraus resultierenden Ungenauigkeiten konnten mit dem Einsatz von Schrägkugellagern mit sehr geringer Rollreibung und kleinem Lagerradius (grosses Verhältnis Rollendurchmesser zu Lagerdurchmesser) sowie Beschränkung der Spannung in den Drähten in zulässigen Grenzen gehalten werden. Auch die in der Prüfhalle auftretenden Temperaturschwankungen von maximal $\pm 1^{\circ} \mathrm{C}$ bewirkten dank der Verwendung von Invar keine unzulässigen Fehler.

\subsection{Versuchsablauf}

Bei allen Versuchen wurde der in Bild 2 festgehaltene Belastungsablauf angewendet. Zuerst wurde die Last jeweils um ein bestimmtes Inkrement gesteigert. Beim Auftreten der ersten Risse wurde eine erste Entlastung bis auf das Eigengewicht vorgenommen. Bei der Wiederbelastung wurde die Last im Bereich des Gebrauchszustandes mit kleinen Inkrementen gesteigert, und es wurden insbesondere sorgfältige Rissmessungen durchgeführt. Bei grösseren Durchbiegungen musste von der lastgesteuerten zur deformationsgesteuerten Belastung übergegangen werden (Verformungsinkrement). Weitere Entlastungen waren jeweils über Nacht vom ersten auf den zweiten Versuchstag nötig.

Während den Messungen wurde auch bei lastgesteuertem Vorgehen die Mittendurchbiegung konstant gehalten (Phass II in Bild 2). Die dabei auftretende Relaxation zeigte sich im Abfallen der Last vom Anfangswert $q_{A}$ auf den Endwert $q_{E}$. Dieser Endwert $q_{E}$ wurde bei der ganzen Auswertung den übrigen Messungen zugeordnet. Wie schon im Abschnitt 2.2 angetönt worden ist, entsprechen den Endlasten die statischen Festigkeitswerte, da sie in ähnlicher Weise erhalten werden.

Da der Hub der Zugkolben für die maximalen Durchbiegungen nicht ausreichte, mussten jeweils zwischen etwa 16 und $18 \mathrm{~cm}$ Mittendurchbiegung die einzelnen Kolben durch Verkürzen der Zugstangen sukzessive nachgestellt werden. Beim Plattenstreifen PS 5 war sogar eine zweite Umstellung bei $26 \mathrm{~cm}$ Mittendurchbiegung erforderlich.

\section{Platte $P$ 1}

Die Anordnung der Messstellen ist in Bild 15 wiedergegeben. Folgende Messungen wurden durchgeführt:

a) Belastung

- Lastablesungen am Federmanometer

- Kontrolle mit Strain Gages auf zwei Zugstangen. 


\section{b) Eckkraft}

- Lastablesung am Pendelmanometer, Konstanthalten der Höhe der Eckpunkte

- Kontrolle mit Strain Gages auf einer Zugstange

- Durchbiegungen zweier diagonal gegenüberliegender Eckpunkte mit induktiven Wegaufnehmern (Anzeige in $0.005 \mathrm{~mm}$ ).

c) Kabelkraftzuwachs

- Messung mit acht Druckmessdosen (Anzeige in $10 \mathrm{~kg}$ ).

d) Verschiebungen

- Photogrammetrische Bestimmung der Lage und der Höhe von 201 Punkten auf der Plattenoberfläche mit zwei Kameras WILD P 31; Durchführung: Institut für Geodär. sie und Photogrammetrie der Eidgenössischen Technischen Hochschule in Zürich $(E T H Z)$.

- Mittendurchbiegung zusätzlich mit drei unabhängigen elektronischen Wegaufnehmern (Anzeige in $0.005 \mathrm{~mm}$ ) und einer mechanischen Uhr (Anzeige in $0.01 \mathrm{~mm}$ ).

e) Betonstauchungen

- Messung mit 24 auf die Plattenoberfläche geklebten Strain Gages, Messlänge $6 \mathrm{~cm}$, entlang einem Mittelschnitt und am Plattenrand (Anzeige in 0.001\% ).

- Die Genauigkeit der photogrammetrischen Messungen reichte nicht aus, um wie angestrebt aus den Relativverschiebungen benachbarter Punkte mit genügender Aussagekraft auf Betondehnungen zu schliessen.

f) Risse

- Rissbreiten an Plattenober- und unterseite mit Rissmassstab (Einschätzen auf $\pm 0.05 \mathrm{~mm}$ ).

\section{Plattenstreifen PS 1 bis PS 5}

Die Anordnung der Messstellen ist in den Bildern 16 und 17 angegeben.

Für die Simulation der seitlichen Feder kamen zwei Verfahren zur Anwendung. Beim Plattenstreifen PS 1 mit starrer seitlicher Haltung $(s=\infty)$ war der Hydro-Pacer so eingestellt, dass er über die Horizontalkolben automatisch die Plattenstreifenlänge konstant hielt. Bei den Versuchen PS 2, PS 4 und PS 5 wurde beim Belasten die Federcharakteristik gemäss der Beziehung

$$
H=s \cdot \Delta I
$$

durch sukzessives Korrigieren der Horizontalkraft approximiert; die Abweichungen von der theoretischen Geraden blieben zu jedem Belastungszeitpunkt unterhalb $5 \%$. Beim Uebergang von Druck- auf Zugkräfte musste jeweils ein kurzer belastungshalt eingeschaltet werden, um die Kolben auf Zug umzustellen.

Folgende Messungen wurden durchgeführt:

a) Belastung

- Lastablesungen am Pendelmanometer

- Kontrolle mit Strain Gages auf zwei Zugstangen. 
b) Horizontalkraft

- Lastablesungen am Federmanometer (Hydro-Pacer) und Vergleich mit Strain Gages auf beiden Pendelstangen erlaubten die Kontrolle der Lagerreibung. Diese betrug weniger als $100 \mathrm{~kg}$.

c) Kabelkraftzuwachs

- Messung mit Druckmessdose am Plattenstreifenende (Anzeige in $10 \mathrm{~kg}$ ).

d) Verschiebungen und Verdrehungen

- Durchbiegungen in 11 Messpunkten mit índuktiven Wegaufnehmern (Anzeige in $0.005 \mathrm{~mm}$ ).

- Mittendurchbiegung zusätzlich mit einer mechanischen Uhr (Anzeige in $0.01 \mathrm{~mm}$ ) und einem weiteren induktiven Wegaufnehmer (Anzeige in $0.005 \mathrm{~mm}$ ).

- Bei allen Durchbiegungsmessungen wurde die Auflagereinsenkung berücksichtigt: Messung mit mechanischen Uhren (Anzeige in $0.01 \mathrm{~mm}$ ).

- Auflagerdrehwinkel mit einem induktiven Neigungsmesser (Anzeige in 0.01 Grad).

8) Betonstauchungen

- Messung mit Strain Gages, Messlänge $6 \mathrm{~cm}$, auf Plattenstreifenoberfläche (Anzeige in $0.001 \%$ ) und

- induktiven Setzdehnungsmessern, Basis $10 \mathrm{~cm}$ (Anzeige in 0.01\%0). Die Setzdehnungsmesser wurden auf Messbolzen aufgesetzt, die mit einem Schnellklebestoff auf der freiliegenden Betonoberfläche aufgeklebt waren; damit konnten die Messinstrumente bis zum Bruch einwandfrei angesetzt werden.

f) Risse

- Rissbreiten mit Rissmassstab (Einschätzen auf $\pm 0.05 \mathrm{~mm}$ ). 


\section{VERSUCHSRESULTATE}

\subsection{Platte P 1}

\subsubsection{Trag- und Bruchverhalten}

Der Belastungsablauf dieses Versuches ist in Tabelle 5 und in den Bildern 18 und 19 wiedergegeben. Nach einem ersten elastischen Teil wurde die Belastungs-Durchbiegungskurve ab Mittendurchbiegungen von etwa $0.6 \mathrm{~cm}$ (Belastung um 6 to $/ \mathrm{m}^{2}$ ) immer flacher und erreichte bei $\theta \mathrm{cm}$ sin erstes relatives Lastmaximum von $9.55 \mathrm{to} / \mathrm{m}^{2}$. Bei $11 \mathrm{~cm}$ Mittendurchbiegung trat ein geringer Lastabfall ein - ein Durchschlagen konnte nicht so ausgeprägt beobachtet werden. Die Platte trug bei $16 \mathrm{~cm}$ die maximale Last von $9.67 \mathrm{to} / \mathrm{m}^{2}$ und kam bei über $26 \mathrm{~cm}$ durch Reissen der Litzen zu Bruch.

Bis zum Auftreten der ersten Risse bei Laststufe 4 verhielt sich die Platte wie eine homogene, elastische Platte, nachher fiel ihre Steifigkeit ab. Bei den folgenden Lastzyklen öffneten und schlossen sich die Risse kontinuierlich. Die Zahl der Risse war beschränkt. An der Plattenunterseite ergaben sich Risse entlang den Plattendiagonalen und einem quadratischen Netz innerhalb der vier mittleren Lasteinleitungspunkte, an der Plattenoberseite senkrecht zu den Diagonalen verlaufende Risse wegen dem Festhalten der Ecken. Mit zunehmender Belastung stellte sich eine leichte Fächerausbildung in den Ecken ein. Aus der anfänglich stetig gekrümmten Durchbiegungsfläche entwickelte sich immer ausgeprägter ein Diagonalmechanismus mit quadratischem Mittelteil, also ein pyramidenstumpfartiges Durchbiegungsfeld. Dementsprechend wuchs die maximale Rissweite in den Diagonalen proportional zur Mittendurchbiegung an. Die Kabelkräfte nahmen gemäss der aus der zunehmenden Plattenverformung resultierenden Litzenverlängerung kontinuierlich zu.

Von'Laststufe 30 an ( $f=6.14 \mathrm{~cm}$ ) konnten entlang den Diagonalen zunehmend Stauchungen an der Plattenoberfläche beobachtet werden. Besonders stark traten diese in der Nähe der äussersten vier Belastungspunkte auf, was bei der entlang den Diagonalen ungefähr konstanten gegenseitigen Rotation der Plattenteile auf grössere Betondruckkräfte in diesem Bereich hinwies. Bei Laststufe 35 ( $f=16.14 \mathrm{~cm}$ ) entstand an der Plattenunterseite ein neuer fast kreisförmig verlaufender Riss durch die innersten Belastungspunkte. Erst zu diesem Zeitpunkt konnten am Rand des quadratischen Mittelteils erste Stauchungen an der Plattenoberfläche festgestellt werden. Beim Versuch, die Eckkraft zu steigern, wurde bei Laststufe 34 eine Plattenecke abgerissen, da die schwache Randarmierung dieser Beanspruchung nicht mehr standhielt. In der Folge konnten die Ecken nicht mehr festgehalten werden, was sich jedoch auf das weitere Tragverhalten unwesentlich auswirkte.

Die gemachten Beobachtungen zeigen, dass eine allmähliche Umlagerung auf ein im Anhang $A$ beschriebenes Tragsystem mit äusserem Betondruckring und aus den Stahllitzen bestehendem Hängwerk stattfand. Freilich ging bis zum Kollaps ein Teil der Betondruckkraft über den inneren Plattenbereich. Es bedarf in diesem Zusammenhang noch einiger Bemerkungen $z u$ der im Anhang $\theta$ berechneten und in Tabelle 11 aufgeführten theoretischen Bruchlast von 11.90 to $/ \mathrm{m}^{2}$. Einerseits traf die Annahme, dass alle Litzen bis zu ihrer Bruchkraft beansprucht waren, in Wirklichkeit nicht zu, und andererseits war wie oben ausgeführt der äussere Druckring nicht voll ausgebildet. Den 
im Platteninnern wirksamen Betondruckkräften entsprachen beim Bruch insgesamt geringe nach unten gerichtete Umlenkkräfte, auf ähnliche Weise, wie dies ausgeprägt beim einfachen Balken PS 3 der Fall war.

Wie im Anhang $\theta$ gezeigt wird, beträgt die Traglast einer Platte mit gleichen Abmessungen und gleicher Armierung - jedoch mit Kabeln im Verbund - $9.60 \mathrm{to} / \mathrm{m}^{2}$. Aus der Tatsache, dess dieser Wert mit der hier experimentell festgestellten Bruchlast vergleichbar ist, sollte kein voreiliger Schluss gezogen werden, da sich ein anderes Tragsystem ausbildete.

\subsubsection{Stahlspannungen}

Bild 20 zeigt ein kontinuierliches, aber unterschiedlich starkes Anwachsen aller gemessener Kabelkräfte mit zunehmender Durchbiegung. Der Zuwachs war erwartungsgemäss bei den mittleren Kabeln am grössten und bei den Randkabeln am kleinsten. Nach dem unmittelbar aufeinanderfolgenden Reissen dreier mittlerer Litzen wurde der Versuch bei $26.75 \mathrm{~cm}$ Mittendurchbiegung abgebrochen. Der bei den Laststufen $37 \mathrm{bzw}$. 38 beobachtete Kraftabfall der Litzen 4 und 14 um etwa 2.5 to ist möglicherweise auf den Bruch eines der sieben Litzendrähte oder auf einen Schlupf in der am Litzenende aufgestauchten Presshülse zurückzuführen.

Das im Anhang B berechnete Mittel der Umlenkkräfte von 11.90 to $/ \mathrm{m}^{2}$ bei einer der Bruchdehnung der Litzen entsprechenden Mittendurchbiegung von $27 \mathrm{~cm}$ basiert auf der Annahme, dess alle Litzen ihre statische Bruchkraft erreichen. Die Bruchkraft wäre bei einer Platte mit Verbund zwischen Stahl und Beton schon bei kleinen Durchbiegungen erreicht. Beim Versuch reichten die aus der Verformung resultierenden Litzendehnungen nur gerade für die mittleren Kabel dazu aus, die Randkabel blieben noch unter der Streckgrenze. Mit dem oben angegebenen Wert werden also die vorhandenen Umlenkkräfte überschätzt, denn er wurde aus einer dem tatsächlichen Spannungs-Dehnungsdiagramm des Stahles umschriebenen Kurve gewonnen.

\section{1 .3 Durchbiegungen}

In Bild 22 ist der Durchbiegungsverlauf in zwei charakteristischen Schnitten entlang den Kabeln 2 und 5 dargestellt. Aus der anfänglich stetig gekrümmten Durchbiegungsform entwickelte sich immer ausgeprägter ein symmetrischer Mechanismus in Form eines Pyramidenstumpfes mit einem quadratischen Mittelteil innerhalb der innersten Lasteinleitungspunkte. Das Aufgehen eines kreisförmigen Risses an der Plattenunterseite bei Laststufe 35 ( $v g l$. Bild 28 ) hatte an der Oberseite eine geringe Vergrässerung des quadratischen Mittelteiles zur Folge und führte zu sichtbaren Betonstauchungen beim Uebergang von diesem zu den geneigten äusseren Plattenteilen (vgl. Bild 29). Bei den Laststufen 37 und 38 traten neue Risse knapp innerhalb der äusseren Belastungspunkte auf (vgl. Bild 26). Damit ergab sich noch ein leichter knick in den vorher ebenen seitlichen Plattenteilen.

Bei der letzten Laststufe konnte eine durch Strecken der Kabel bewirkte schwache Gegenkrümmung des mittleren Plattenteiles beobachtet werden. Besonders schön trat dieselbe Erscheinung zwischen den Lasteinleitungsstellen des PS 5 auf (vgl. Bild 84). 


\subsubsection{Betonstauchungen}

Bild 23 zeigt die Entwicklung der Betonstauchungen an einzelnen Messstellen mit zunehmender Durchbiegung. Aus Bild 24 ist der Stauchungsverlauf über einen Mittelschnitt ersichtlich.

Die Messstellen 413 und 415 lagen über den an der Plattenunterseite ein Quadrat von ca. $60 \mathrm{~cm}$ Seitenlänge bildenden Rissen (vgl. Bild 25). Die Stauchungen an diesen beiden Messstellen wuchsen bis zur Laststufe 33 ( $f=9.14 \mathrm{~cm}$ ) stetig an, während alle anderen Messungen im Mittelschnitt praktisch auf einem bald ( $f=1.53 \mathrm{~cm}$ ) erreichten Wert verharrten. Mit dem Auftreten des wiederholt erwähnten Kreisrisses bei Laststufe 35 verlagerte sich das Rotationszentrum nach aussen, was zu grösseren Stauchungen an den Messstellen 412 und 416 sowie zu sichtbaren Aufstauchungen längs den Seiten eines Quadrates von ca. $105 \mathrm{~cm}$ Seitenlänge Anlass gab (vgl. Bild $27 \mathrm{c}$ ). Der bei den Laststufen 37 und 38 sich ausbildende leichte Knick entlang den äusseren 12 Belastungspunkten schlug sich im Anwachsen der entsprechenden Betonstauchungen nieder (Messstellen 410 und 418 ).

Bei einem Pyramidenstumpfmechanismus einer Quadratplatte verhalten sich die Rotationen entlang den Diagonalen zu denjenigen entlang den Nahtstellen zwischen inneren und äusseren Plattenteilen wie $\sqrt{2}: 1$. Wären die Betondruckkräfte in beiden Hauptrichtungen gleichmässig über die Plattenbreite verteilt, so müssten sich die dem Stauchungsbeginn an diesen Stellen entsprechenden Mittendurchbiggungen wie 1: $\sqrt{2}$ verhalten. Beim Versuch konnten erste Stauchungen in den Diagonalen deutlich bei Laststufe 30 und am Rand des quadratischen Mittelteiles bei Laststufe 35 beobachtet werden. Das zugehörige Durchbiegungsverhältnis von 1:2.5 dürfte somit auf grössere Betondruckkräfte im äusseren Teil der Diagonalen zurückzuführen sein. Diese Bemerkung wird weiter belegt durch einen Vergleich der zu den Laststufen 34 und 35 (Durchbiegungsverhältnis ca. $1: \sqrt{2}$ ) gehörigen Bilder 27 b und 27 c. Die Diagonalenstauchungen bei Laststufe 34 sind viel ausgeprägter als die Stauchungen entlang den Quadratseiten bei Laststufe 35. Schliesslich zeigen die Bilder 27 d und 29 sehr grosse Stauchungen bei Laststufe 37 , vor allem im äusseren Bereich der Diagonalen.

Wie aus Bild 23 hervorgeht, erfuhren die sechs in der Mitte des Plattenrandes angeordneten Strain Gages zunächst eine geringfügige Zugdehnung, wurden dann aber bei grösseren Durchbiegungen deutlich gestaucht.

Die vorhandenen Beobachtungen lassen eine Umlagerung auf ein Tragsystem erkennen, das dem im Anhang A beschriebenen Modell mit äusserem Betondruckring und Stahlhängewerk ähnlich ist. Ein Teil der Betondruckkraft ging bis zum Bruch über den inneren plattenbereich.

\subsubsection{Rissverhalten}

Die Bilder 25 und 26 geben einen Ueberblick über den Verlauf und die Grösse der Risse.

Die ersten Risse an der Plattenunterseite öffneten sich entlang den Diagonalen und einem quadratischen Netz innerhalb der vier mittleren Lasteinleitungspunkte. An der Oberseite ergaben sich bei den Ecken senkrecht zu den Plattendiagonalen verlaufende Risse. Die Differenz zwischen der in Tabelle 11 aufgeführten theoretischen Risslast und dem bei Laststufe 4 experimentell festgesteliten wert ist einerseits mit dem in 
der Rechnung vernachlässigten Einfluss der Löcher bei den Lasteinleitungspunkten zu erklären und andererseits mit dem vergleichsweise hohen und stark streuenden Wert für die Biegezugfestigkeit (vgl. Tabelle 4).

Bei den nachfolgenden Lastzyklen öffneten und schlossen sich die Risse kontinuierlich, und die experimentelle Risslast stimmte mit der theoretischen sehr gut überein ( $v g l$. Bild 21 und Tabelle 11).

Mit grösseren Durchbiegungen ergab sich eine leichte Fächerausbildung in den Ecken, und die maximale Rissweite in den Diagonalen nahm proportional zur Mittendurchbiegung zu. Der bei Laststufe 35 entstandene Kreisriss und die bei den Laststufen 37 und 38 sich entwickelnden Risse entlang den äusseren 12 Belastungspunkten wurden bereits weiter oben beschrieben ( $v g l$. Bild 28 ).

Bild 21 dokumentiert das Rissverhalten der Platte $P 1$ bei kleinen Durchbiegungen (Gebrauchszustand). Zum Vergleich ist noch die aufgebrachte Belastung angegeben. Interessant ist der Vergleich der maximalen Rissweite an plattenunterseite mit den entsprechenden Kurven der Plattenstreifen, wie er in den Bildern 78 und 79 durchgeführt worden ist. Die Kurven der Platte P 1 liegen in beiden Darstellungen zwischen denen der Plattenstreifen PS 2 (mittlere seitliche Haltung) und PS 3 (einfacher Balken).

\subsection{Plattenstreifen PS 1 - PS 5}

\subsubsection{Trag- und Bruchverhalten}

Bei allen fünf Plattenstreifenversuchen konnte das ganze Spektrum der Traggeschichte beobachtet werden: Gebrauchszustand, Grenzzustand bezüglich Verformungen und Risse sowie Grenzzustand Bruch. In den Tabellen 6 bis 10 sind für alle Laststufen die Mittendurchbiegungen, die Anfangs- und Endlasten, die Horizontalkräfte resp. beim PS 3 die Horizontalverschiebungen zusammengestellt. Die Bilder 30 bis 39 zeigen die Endlasten in Funktion der Mittendurchbiegung. Für jeden Versuch wurden der ganze Verlauf mit dem letzten Lastanstieg, d.h. nach ein- oder mehrmaligem Entlasten (Bilder 30, 32, 34, 36, 38) und der Anfangsbereich mit sämtlichen Laststufen (Bilder 31, 33, 35, 37 , 39) getrennt aufgetragen. Die Last-Durchbiegungskurven aller Versuche sind in $\theta i l d 40$ zusammengestellt.

Wie aus Bild 31 ersichtlich ist, wurde der Plattenstreifen PS 1 zweimal bis über die Risslast hinaus belastet und entlastet. Man sieht, dass sich die Biegesteifigkeit vor allem vom ersten Lastanstieg, bei dem der Plattenstreifen ungerissen war, zum zweiten Anstieg bei gerissenem Plattenstreifen am stärksten verringerte. Beim zweiten und dritten Lastanstieg änderte sich die Biegesteifigkeit nur mehr unmerklich. Darum wurden die Plattenstreifen PS 2 bis PS 5 nur mehr einmal über die Risslast hinaus belastet und entlastet.

Bis zur Risslast war das Verhalten aller Plattenstreifen elastisch und entsprach im wesentlichen demjenigen eines einfachen Balkens. Mit Ausnahme von PS 3 , der seitlich nicht gehalten war, zeigten alle Plattenstreifen nach Erreichen eines ersten relativen Lastmaximums ein mehr oder weniger ausgeprägtes Durchschlagen. Nach einem Abfall der Last auf ein relatives Lastminimum folgte anschliessend bei den Plattenstreifen 
PS 1, PS 2, PS 4 und PS 5 ein stark ansteigender, fast linearer Belastungsanstieg. Dieser Anstieg ist bedingt durch die Durchbiegungen (Theorie 2. Ordnung) und entspricht ungefähr dem Verhalten eines Seilwerkes, wenn der Stahl fliesst.

Der Bruch trat bei allen Versuchen durch Reissen der Litzen ein. Die maximal auftretende Mittendurchbiegung $f_{\text {max }}$ variierte bei den Plattenstreifen PS 1 bis PS 4 zwischen $24.6 \mathrm{~cm}$ und $26 \mathrm{~cm}$, was gut mit dem theoretischen Wert übereinstimmte. Beim PS 5 hingegen trat der Bruch schon bei $35 \mathrm{~cm}$ ein. Da der theoretische Wert etwa $47 \mathrm{~cm}$ betrug, wurde beim PS 5 die im Vorversuch gemessene Bruchdehnung der Litzen nicht erreicht.

In die Belastungs-Durchbiegungskurven wurden folgende numerische Werte eingetragen: Eigengewicht $g$, Ausgleichslast $u$, Vergleichsbruchlast $q_{u v}$, wenn Litzen im Verbund wären, relatives Lastmaximum $q_{1}$, relatives Lastminimum $q_{2}$ und Bruchlast $q_{u}$. Es sei hier nochmals darauf hingewiesen, dass der $q-f-V e r l a u f$ bis gegen $q_{1}$ kraftgesteuert und dann deformationsgesteuert bestimmt wurde. Bei einer Kraftsteuerung über den ganzen Bereich würde der Durchschlag schlagartig vor sich gehen. Im Nachfolgenden soll die Last-Durchbiegungskurve der einzelnen Versuche kurz diskutiert werden.

\section{Plattenstreifen PS 1}

Als charakteristischer. Parameter wurde bei diesem Versuch die seitlich starre Haltung $(s=\infty)$ gewählt. Das erste relative Lastmaximum $q_{1}$ wurde bei einer Durchbiegung von $2.17 \mathrm{~cm}$ erreicht. Das Verhältnis $q_{1} / q_{u v}$ betrug 0.95 und $q_{1} / q_{2}$ war 1.51 . Der Durchschlageffekt, sichtbar in der grossen Lastabnahme von $q_{1} z u q_{2}$, war ausgeprägt. Die Horizontalkraft H. (vgl. Bild 47) betrug im Maximum 27.2 to auf Druck und 20.9 to auf Zug. Dass der im Zugbereich erreichte Wert grösser als die Bruchlast der Litze war, kann dadurch erklärt werden, dass die beiden oben eingelegten Längseisen ebenfalls Zugkräfte aufnahmen. Diese Tatsache ist auch aus dem Vergleich $q_{u}^{T h}$ und $q_{u}^{E x}$ in Tabelle 11 ersichtlich.

\section{Plattenstreifen PS 2}

Der Plattenstreifen PS 2 stellte den eigentlichen Standardversuch dar. Die Steifigkeit der seitlichen Feder betrug $7 \cdot 10^{3}$ to/m. Das erste relative Lastmaximum wurde bei einer Durchbiegung von $3.2 \mathrm{~cm}$ erreicht. Das Verhältnis $q_{1} / q_{u v}$ betrug 0.93 . Wie aus dem Verhältnis $q_{1} / q_{2}$ von $1.35 \mathrm{zu}$ sehen ist, schwächte die elastische seitliche Haltung den Durchschlagseffekt ab. Die Horizontalkraft H ( $v g l$. Bild 48) betrug auf Druck im Maximum 13 to, was etwa die Hälfte vom Wert $H$ beim PS 1 darstellte, und 20.7 to auf Zug. Auch hier kamen die Montageeisen in der Schlussphase zum Tragen.

\section{Plattenstreifen PS 3}

Dieser Plattenstreifen mit einer seitlichen Feder von $s=0$ war statisch gesehen ein einfacher Balken. Beim ersten Belastungsanstieg fiel beim Auftreten des ersten Risses die Last leicht ab. Beim zweiten Belastungsast jedoch konnte ein kontinuierliches Anwachsen der Last bis zu einem Fliessplateau festgestellt werden. Das Verhälnis $q_{1} / q_{u v}$ betrug 0.9 , wobei dieses Verhältnis nicht mit denjenigen der anderen Versuche verglichen werden kann. Der Bruch trat auch hier erst durch Reissen der Litze bei einer Mittendurchbiegung von $26 \mathrm{~cm}$ auf. Erstaunlich war die sehr grosse Rotations- 
fähigkeit, die bei diesem Versuch beobachtet werden konnte. Nach der Modellvorstellung hätte die Last $q_{u v}$ erreicht werden sollen. Dass dies nicht ganz der fall war, kann dadurch erklärt werden, dass bei einer Durchbiegung von 5 bis $10 \mathrm{~cm}$ die Stahlspannung gemäss $\sigma-\varepsilon-D i a g r a m m$ oder auch nach gemessener Kabelkraftzunahme $\Delta V$ die Bruchspannung noch nicht erreichte. Bei den grösseren Durchbiegungen wurde der Hebelarm der inneren Kräfte durch Abblättern der äussersten Betonfaser verkleinert. Die theoretische Tragfähigkeit wurde darum nicht ganz erreicht, trotzdem die maximale Bruchkraft im Stahl vorhanden war. Aus Bild 49, in dem die Längenänderung des Plattenstreifens aufgetragen wurde, kann man deutlich sehen, wie das Betongewölbe in der Anfangsphase eine Verlängerung und ab einer gewissen Durchbiegung das Stahlhängewerk eine Verkürzung des Plattenstreifens bewirkte.

\section{Plattenstreifen PS 4}

Der Plattenstreifen PS 4 entsprach mit Ausnahme einer zusätzlichen schlaffen Armierung von $\mu=0.21 \%$ dem PS 2. Das Verhältnis $q_{1} / q_{u v}$ betrug 0.87 und $q_{1} / q_{2}$ war 1.07 . Aus dem Verhältnis $q_{1} / q_{2}$ kann man ersehen, dass sich der Durchschlagseffekt gegenüber PS 2 verringerte. Ab einer Mittendurchbiegung von $6 \mathrm{~cm}$ war die Tragfähigkeit gegenüber PS 2 um einen Anteil grösser, der dem plastischen Moment infolge schlaffer Armierung entsprach. Bei einer Durchbiegung von $23 \mathrm{~cm}$ wurde das absolute Lastmaximum von $q=5.25$ to/m erreicht. Zu diesem Zeitpunkt trugen Vorspannstahl und schlaffe Armierung gemeinsam als Hängewerk. Der brüske Lastabfall ergab sich durch einen Verankerungsbruch der schlaffen Armierung beim Stoss mit den an der Endplatte angeschweissten Bügeln. Die Bruchlast $q_{u}$ von 4.4 to/m ergab sich wiederum aus dem Anteil Hängewerk des Vorspannstahles und dem Biegeanteil, der dem plastischen Moment infolge schlaffer Armierung entspricht. Die Horizontalkraft betrug auf Druck im Maximum 9.2 to und auf Zug 27.7 to, resp. 18.7 to nach dem Verankerungsbruch der schlaffen Armierung.

Bei einem Vergleich der Plattenstreifen PS 2 und PS 4 kann gesagt werden, dass durch die schlaffe Armierung der PS 4 stärker den Charakter eines Biegebalkens annahm. Es ist zu beachten, dass beim ersten relativen Lastmaximum $q_{1}$ die Last $q_{E}$ beim PS 4 gegenüber dem PS 2 nicht um den vollen Biegeanteil vergrössert war, der dem plastischen Moment infolge $F_{e}$ entsprechen würde, obwohl die schlaffe Armierung bereits ins Fliessen kam. Das heisst aber, dass das Betongewölbe in dieser Phase beim PS 4 weniger zum Wirken kam als beim PS 2, was auch aus der Verringerung der Horizontalkraft $H$ ersichtlich ist.

\section{Plattenstreifen PS 5}

Der charakteristische Parameter beim Plattenstreifen PS 5 war die Schlankheit, die gegenüber den anderen Versuchen auf 1/33, resp. 1/47 vergrössert wurde. Bei der Laststufe 4 wurde versehentlich eine zu grosse Mittendurchbiegung aufgebracht. Die Risse konnten sich bei der Entlastung nicht mehr ganz schliessen, was eine bleibende Durchbiegung von etwa $0.5 \mathrm{~cm}$ zur Folge hatte. Das Verhältnis $q_{1} / q_{u v}$ betrug 0.76 . Aus dem Verhältnis $q_{1} / q_{2}$ von 1.02 sieht man, dass die grössere Schlankheit bewirkte, dass der Durchschlagseffekt sehr schwach auftrat. Das erste relative Lastmaximum lag beim PS 5 im Vergleich zu den anderen Plattenstreifen am weitesten unter der mit Verbund gerechneten Vergleichslast $q_{u v}$ (vgl. Tab. 11). Bei einer Durchbiegung von $26 \mathrm{~cm}$ bewirkte das Umstellen der Zugstangen einen Lastabfall. Der Lastabfall bei einer Mittendurchbiegung von $30 \mathrm{~cm}$ entstand beim schlagartigen Deffnen eines Risses in der Nähe des Auflagers. 


\subsubsection{Stahlspannungen}

Ein wesentliches Ziel der durchgeführten Versuche war es, genauere experimentelle Resultate über den Verlauf der Stahlspannungen in den Vorspannlitzen zu gewinnen. In den Bildern 41 bis 45 ist der Zuwachs der Kabelkraft in Funktion der Mittendurchbiegung einzeln dargestellt. Bild 46 zeigt einen Vergleich der fünf Plattenstreifen, wobei die Durchbiegung auf die Spannweite 1 normiert wurde.

Als wichtigstes Ergebnis kann festgehalten werden, dass bei allen Versuchen, auch beim einfachen Balken PS 3 , die Kabelkraft von $V_{0}$ kontinuierlich bis zum Bruch angewachsen ist. Die leichten Knicke bei einer Mittendurchbiegung um $18 \mathrm{~cm}$ traten jeweils beim Nachstellen der Zugkolben auf, waren also versuchstechnisch bedingt. Der Bruch kam immer durch Reissen der Litzen bei den Verankerungen zustande. Die aufgepressten Hülsen hatten dort eine ganz leichte Schwächung des Drahtmaterials zur Folge. Die erreichten Bruchdehnungen stimmten bei. den Plattenstreifen PS 1 bis PS 4 genau mit der im Vorversuch gemessenen überein. Beim Plattenstreifen PS 5 hingegen wurde die erwartete Bruchdehnung nicht erreicht. Anstelle von $2.66 \%$ betrug die stahlbruchdehnung ca. $2 \%$. Die Bruchkraft der Litzen variierte zwischen 16.7 und 17.82 to (im Vorversuch bestimmte statische Bruchlast: 16.77 tol.

Da die bei der Verankerung gemessene Kabelkraft genau mit der zu erwartenden Bruchkraft übereinstimmte, kann gesagt werden, dass praktisch keine Reibung vorhanden war, denn bei vorhandener Reibung müsste die Kabelkraft in Feldmitte am grössten sein und damit über der Bruchlast liegen. Dies wurde auch dadurch bestätigt, dass jeweils nach dem Versuch die eingefetteten Litzen von Hand aus dem immer noch verformten Plattenstreifen herausgezogen werden konnten.

Ein Vergleich der fünf Kurven ( $v g l$. Bild 46) zeigt, dass im Anfangsbereich ( 0 bis $2.5 \mathrm{~cm}$ Mittendurchbiegung) der Kabelkraftzuwachs in der Reihenfolge PS 5, PS 4, PS 1, PS 2 und PS 3 stärker zunimmt. Dies stimmt mit der Ueberlegung überein, dass das Betongewälbe je nach seitlicher Haltung das Spannglied mehr oder weniger verlängern konnte.

\section{$\underline{4.2 .3 \text { Durchbiegungen }}$}

Der Verlauf der Durchbiegungen ist für eine Auswahl von Laststufen in den Bildern 53 bis 57 für alle Versuche dargestellt. Für die Laststufeñ mit kleinen Durchbiegungen zeigten alle Plattenstreifen eine fast stetige Krümmung. Von einer gewissen Durehbiegung an begann sich ein Gelenk, meistens unter einem Lasteinleitungspunkt, auszubilden. Im Verlauf der weiteren Belastung ergab sich ein regelrechtes Hin- und Herpendeln zwischen zwei oder drei Rotationspunkten bis zum Bruch. Beim einfachen Balken PS 3 bildete sich gegen Schluss nur noch ein Rotationspunkt aus. Die zwischen den einzelnen Gelenken liegenden Teile verhielten sich in dieser Phase, mit Ausnahme von PS 4, fast starr. Die anfängliche Krümmung bildete sich sogar zurück. Zufolge der schlaffen Armierung blieb beim Plattenstreifen PS 4 bis zum Schluss eine gewisse Krümmung über die ganze Spannweite erhalten. Der Hauptteil der Verformung resultierte aber auch beim PS 4 aus Rotationen in einigen Gelenken. 


\subsubsection{Betonstauchungen}

In den Bildern 58 bis 62 wurde der Verlauf der Betonstauchungen einzelner Messstellen in Funktion der Durchbiegungen dargestellt. Für verschiedene Laststufen wurden die Betonstauchungen über die ganze Balkenlänge aufgetragen (vgl. Bilder 63 bis 67). Die unter 4.2.3 beschriebenen Beobachtungen zeigten sich auch bei der Entwicklung der Betonstauchungen. Bei den letzten Laststufen wurden die Betonstauchungen nicht mehr gemessen, da zu diesem Zeitpunkt, mit Ausnahme von PS 3, die Last bei allen Plattenstreifen praktisch voll über die Hängewirkung des Spannstahles abgetragen wurde.

Bei den Einzelmessstellen, unter denen sich nicht direkt ein Gelenk ausbildete, konnte man zuerst ein Anwachsen der Betonstauchungen beobachten. Dies entsprach dem Ausbilden einer kontinuierlichen Krümmung. Bei grösseren Durchbiegungen nahmen die Stauchungen wieder ab. Dies zeigte sich am ausgeprägtesten bei den Plattenstreifen PS 1 , PS 2 und PS 5, was wiederum auf ein Zurückbilden der anfänglichen Krümmung und dadurch auf ein starr-plastisches Vertialten der Plattenstreifen hinwies. Beim einfachen Balken PS 3 war dies darum nicht der Fall, weil die Druckkraft, die hier der Zugkraft im Spannstahl entsprechen musste, voll im Beton verblieb.

Bei den grössten Betonstauchungen über den sich ausbildenden Betongelenken löste sich bei Stauchungen von ca. $4 \%$ die äusserste Betonschicht ab, und es begann sich ein richtiges Betongelenk, etwa vergleichbar mit zwei aufeinander abrollenden knochen, auszubilden, wie dies in Bild $83 \mathrm{zu}$ sehen ist. Diesen beobachteten Vorgang kann man nicht mehr als übliches Betonstauchen bezeichnen, sondern es ist ein gegenseitiges Abrollen zweier starrer Teile. Dadurch entsteht eine fast unbeschränkte Rotationsfähigkeit, wie sich dies speziell bei dem als einfachen Balken gelagerten Plattenstreifen PS 3 gezeigt hatte. Selbstverständlich hängt diese Rotationsfähigkeit auch mit der erforderlichen Grösse der Betondruckzone zusammen, die beim PS 3 etwa $2 \mathrm{~cm}$ betrug, also vergleichbar mit der Abmessung des grössten Korndurchmessers des Betons war. Bei Platten aber bewegt sich die Betondruckzone immer etwa in dieser Grössenordnung.

Wenn man die über die Balkenlänge aufgetragenen Betonstauchungen der einzelnen Versuche vergleicht, so sieht man, dass beim PS 1 und PS 4 eine ähnlich gute Verteilung der Stauchungen über die Balkenlänge aufgetreten ist. Die bessere seitliche Haltung beim PS 1 bewirkte eine ähnliche Verteilung der Betonstauchungen und somit auch eine ähnliche Verteilung der Krümmung wie das Zulegen einer schlaffen Armierung beim PS 4. Die gleiche Feststellung werden wir wieder beim Besprechen der Risse antreffen. Beim PS 3 und PS 5 konzentrierte sich die Betonstauchung bereits von kleinen Durchbiegungen an hauptsächlich über den einzelnen Gelenken. Eine etwa dazwischenliegende Verteilung zeigten die Stauchungen des Plattenstreifens PS 2.

Die beobachteten Betondehringen und entsprechenden negativen Krümmungen hingen mit der seitlichen Haltung, den Umlenkkräften aus Vorspannung und der Anordnung der Belastung zusammen. 


\subsubsection{Rissverhalten}

Die Rissausbildung und die Rissgrössen der fünf Versuche sind in den Bildern 68 bis 72 und 80 bis 84 dargestellt. In den Bildern 73 bis 77 sind die maximalen Rissweiten und die Belastung für kleine Durchbiegungen in Funktion der Mittendurchbiegung aufgetragen. Es sei nochmals darauf hingewiesen, dass beim Plattenstreifen PS 5 ( $v g$ l. Bild 77) beim ersten Belastungszyklus versehentlich eine zu grosse Durchbiegung aufgebracht wurde. Der maximale Riss konnte sich wegen der gegenseitigen Verschiebung der muscheligen Betonoberflächen bei der Entlastung nur noch bis auf $0.2 \mathrm{~mm}$ schliessen. In Bild 77 wurde zur gemessenen maximalen Rissöffnung eine strichlierte Kurve eingetragen, die bei der theoretischen Risslast unter der Annahme, dass die Betonbiegezugfestigkeit null sei, resp. der entsprechenden Durchbiegung, beginnt. In den weiteren Vergleichsbildern wurde nur mehr die korrigierte Kurve verwendet.

Die Entwicklung der Risse wurde bis zum ersten Lastmaximum und dadurch im Bereich mäglicher Gebrauchslasten sehr detailliert beobachtet und gemessen. Darum wurde in diesem Bereich beim letzten Lastanstieg mit sehr kleinen Lastschritten vorgegangen.

Vergleicht man die Anzahl Risse der verschiedenen Plattenstreifen, so sieht man, dass beim PS 1 zwei bis sieben, beim PS 2 zwei bis fünf, beim PS 3 ein bis drei, beim PS 4 zwei bis zwanzig und beim PS 5 zwei resp. erst sehr spät fünf Risse aufgetreten sind. Die Stärke der Betondruckzone, von der Risswurzel bis zur äusseren Betonschicht gemessen, betrug 2 bis $4 \mathrm{~cm}$.

In Bild 78 sind die maximalen Rissweiten in Funktion der auf die Spannweite normierten Durchbiegungen aufgetragen. Diese interessante Darstellung zeigt gut den Einfluss der seitlichen Feder, der schlaffen Armierung und der Schlankheit auf das Rissverhalten. Man sieht, dass sich die Plattenstreifen PS 4 und PS 1 bezüglich Risse am besten verhielten. Die bessere seitliche Haltung beim PS 1 zeigte bei kleinen Durchbiegungen etwa die gleiche Wirkung auf das Rissverhalten wie das Zulegen einer schlaffen Armierung beim PS 4. Der als einfacher Balken gelagerte Plattenstreifen PS 3 zeigte das schlechteste Verhalten. Die Plattenstreifen PS 2 und PS 5 liegen dazwischen, wabei sich aber der PS 2 wesentlich besser verhielt.

In Bild 79 wurde die maximale Rissweite in Funktion der auf $q_{u v}$ normierten Last aufgetragen.

Als wesentlichstes Resultat der Rissbeobachtung kann festgestellt werden, dass sich die Risse bei allen Versuchen und bei allen Lastzyklen kontinuierlich und nicht schlagartig öffneten. Durch entsprechende seitliche Haltung oder Zulegen einer schlaffen Armierung kann das Rissverhalten wirkungsvoll beeinflusst werden. 
Im Rahmen des Forschungsprojektes "Vorgespannte Platten" wurden am Institut für Baustatik und Konstruktion der Eidgenössischen Technischen Hochschule in Zürich Versuche an einer Quadratplatte und fünf Plattenstreifen durchgeführt. Die Versuche hatten zum Ziel, das Biegeverhalten von vorgespannten, nichtinjizierten Platten im Gebrauchsund Bruchzustand experimentell zu untersuchen. Hauptparameter für die Serie der Plattenstreifen waren die seitliche Haltung, zusätzliche schlaffe Armierung und die Plattenschlankheit (vgl. Tabelle 1). Nebst der aufgebrachten Last wurden Membrankräfte, Spannkraftzunahme, Durchbiegungen, Betonstauchungen, Rissverlauf und Rissbreiten gemessen.

Die Ergebnisse dieser Versuche lassen sich wie folgt zusammenfassen:

- Bei der Quadratplatte zeigte sich eine allmähliche Umlagerung auf ein Tragsystem mit äusserem Betondruckring und Hängewerk der Vorspannlitzen, ähnlich dem im Anhang $A$ beschriebenen Modell, wobei ein Teil der Betondruckkräfte bis zum Bruch über den inneren Plattenbereich übertragen wurde. Es bildete sich ein ausgeprägter Diagonalmechanismus mit quadratischem Mittelteil entsprechend der Belastungsanordnung und leichten Eckfächern aus.

- Bei allen Plattenstreifen, mit Ausnahme des einfachen Balkens PS 3 , stieg die Last mit zunehmender Durchbiegung bis zu einem ersten relativen Maximum an, fiel dann auf ein Minimum zurück, um erneut, entsprechend dem Verhalten eines Seilwerkes, bis zum Bruch anzuwachsen. Dieses für Durchschlagsprobleme charakteristische Verhalten bestätigte im wesentlichen des im Anhang A beschriebene Modell. Beim Plattenstreifen PS 3 (einfacher Balken) ging die Belastungs-Durchbiegungskurve nach einem elastischen Anstieg in ein ausgeprägtes Fliessplateau über.

- Der Bruch trat bei allen Versuchen, auch beim einfachen Balken PS 3, durch Reissen der Litzen ein und nicht durch Versagen der Betondruckzone. Ausser beim PS 5 entsprachen die maximalen Durchbiegungen der in Zugversuchen ermittelten Bruchdehnung der Vorspannlitzen. Als Konsequenz der grossen Verformungen war bei allen Versuchskörpern die geleistete Qissipationsarbeit beachtlich.

- Ein Vergleich der experimentellen Bruchlast mit der theoretischen Traglast von sonst gleichen Versuchskörpern, aber mit Litzen im Verbund, zeigt folgendes Bild: Bei der Platte P 1 und beim Plattenstreifen PS 3 war die experimentelle Bruchlast etwa gleich gross wie dieser Bezugswert, bei den übrigen Plattenstreifen hingegen zwei bis dreieinhalb mal so gross.

- Die Zugkräfte in den Vorspannlitzen wuchsen bei allen Versuchen kontinuierlich bis zum Bruch an.

- Die Verteilung der Betonstauchungen war stark von der Steifigkeit der seitlichen Haltung und der zusätzlichen schlaffen Armierung abhängig. Beim einfachen Balken PS 3 ergab sich nach Abblättern der äussersten Betonschicht eine gegenseitige Rotation der Balkenteile ohne merklichen Verlust der Tragfähigkeit bis zum Litzenbruch. Diesen Vorgang kann man sich etwa mit dem Bild zweier aufeinander abrollender knochenenden veranschaulichen. 
- Die Risse öffneten sich stets kontinuierlich und nicht schlagartig. Durch die seitliche Haltung und das Zulegen einer schlaffen Armierung konnte das Rissverhalten bezüglich Rissbreite und -verteilung wirkungsvoll beeinflusst werden.

- Bei einer Bemessung müssen zwei Grenzzustände betrachtet werden: Beeinträchtigung der Nutzung durch Verformungen und Risse sowie Verlust der Tragfähigkeit beim Bruch. Während bei systemen in Verbund normalerweise eine genügende Bruchsicherheit auch ein ausreichend gutes Verhalten im Gebrauchszustand gewährleistet, wird bei den hier untersuchten Systemen ohne Verbund der Grenzzustand der Nutzung oft massgebend. 
Dans le cadre d'un programme de recherche sur le comportement des dalles précontraintes, on a procéde, a l'institut de Statique et de Construction de l'Ecole Polytechnique Fédérale de Zurich (EPFZ), à des essais sur une dalle carrée, simplement appuyée et sur cinq bandes d'une plaque allongée. Le but de ces essais était l'étude expérimentale du comportement à la flexion des dalles précontraintes avec des câbles non injectés dans l'état d'utilisation et dans celui de rupture. Pour les bandes, les paramètres principaux comprenaient les conditions d'appuis horizontaux, l'armature conventionnelle et l'élancement (voir tableau 1). En plus de la charge appliquée on a mesuré les actions de membrane de compression et de traction, l'augmentation de la force de précontrainte, la flèche, la répartition et la largeur des fissures, ainsi que les déformations du béton.

Les résultats des essais peuvent se résumer de la façon suivante:

- Pour la dalle carrée on a pu constater une redistribution successive des efforts sur un système statique, formé d'un anneau extérieur comprimé constitué par le béton et d'une membrane de traction due aux câbles précontraints. Ce comportement du système peut s'expliquer par le modèle decrit dans l'annexe A. Une partie de la force de compression du béton s'est transmise, jusqu' au moment de la rupture, vers la partie centrale de la dalle. Le méchanisme général de rupture avait la forme de tronc de pyramide et était complété, dans les angles, par des méchanismes locaux en éventail.

- Pour toutes les bandes, à l'exception de celle portant le numéro PS 3, la charge et la flèche augmentèrent jusqu' à un premier maximum, puis diminuèrent jusqu' à un minimum pour recommencer à augmenter jusqu' à la rupture, d'une façon analogue à celle d’un ouvrage suspendu. Ce comportement caractéristique est semblable au problème de stabilité où la déformation finale est inverse à la déformation initiale et correspond pour l'essentiel au modèle décrit dans l'annexe A. Pour la bande PS 3 , considérée comme poutre simple, le diagramme charge-flèche augmenta proportionellement pour atteindre le plateau d'écoulement.

- L'état limite est atteint pour tous les essais y compris la poutre simple PS 3 , par rupture des torons précontraints et non par écrasement du beton. A I'exclusion de la bande PS 5, les flèches maximales correspondèrent à l'allongement de l'acier mesuré lors des essais de traction. Une conséquence des grandes déformations est que le travail de dissipation fut important.

- La comparaison des charges de rupture déterminées expérimentalement avec celles établies par le calcul pour des bandes précontraintes avec injection nous donne les résultats suivants: Pour la plaque carrée et la bande PS 3 les charges expérimentales avaient le même ordre de grandeur que celles etablies par le calcul. Pour les autres bandes, par contre, le rapport entre la charge expérimentale de rupture et la charge théorique variait entre 2 et 3,5 .

- Les efforts de traction dans les torons augementèrent continuellement jusqu' à la rupture. 
- La répartion des déformations du béton dépendit fortement de la rigidite des appuis latéraux ainsi que du pourcentage d'armature conventionelle. Pour la poutre simple PS 3, il se forma, après l'écrasement des fibres exterieures, une rotule autaur de laquelle les portions de la poutre pivotèrent, sans pour autant diminuer la capacité portante, jusqu' à la rupture des torons. Ce phénomène est semblable à celui que subissent deux os tournant autour de leur articulation.

- Louverture des fissures s'effectua d'une maniere continue et non pas brutale. La répartion et l'ouverture des fissures fut fortement influencé par les appuis latéraux et par l'armature conventionelle.

- Pour effectuer un dimensionnement d'une structure on doit considerer deux états limites: celui de l'etat d'utilisation et celui de la rupture. Pour les systèmes précontraints avec injection on obtient habituellement, avec une sécurité à la rupture suffisante, aussi un comportement admissible de l'ouvrage par rapport aux déformations et à la fissuration. Tel n'est pas le cas pour les systèmes précontraints sans injection pour lesquels le critère d'utilisation de l'ouvrage est souvent déterminant. 
A square plate and five platestrips were tested at the Institute of Structural Engineering, Swiss Federal Institute of Technology (ETH), Zurich, as part of a research program to study the behavior of prestressed concrete slabs. The primary objective of these tests was to investigate the behavior in flexure of unbonded prestressed concrete slabs under working and failure load. The main parameters for the platestrip specimens (restraint against lateral movements, supplementary bonded reinforcement, span-depth ratio) are given in Table 1 . At each stage of the tests membrane forces, forces in the prestressing tendons, deflections, concrete strains, crack patterns, crack widths and applied loads were recorded.

The results of these tests may be summarized as follows:

- For the simply supported square slab tensile membrane action of the network consisting of the tendons and the surrounding concrete compression ring (compare Appendix A) became more and more important with increasing deflections. The failure mechanism had the shape of a truncated pyramid with a square top within the innermost loading points remaining horizontal and small fans at the corners.

- The load-deflection curves of all platestrips except the simply supported beam PS 3 showed a typical snap-through behavior. The applied load increased up to a relative maximum with increasing deflection, decreased then to a minimum and increased again according to the behavior of a network. This confirmed the usefulness of the theoretical model taken as a basis for the present investigation. The applied load on specimen PS 3 reached a typical yield plateau after the elastic range.

- All specimens failed by rupture of the tendons and not by crushing of the concrete compression zone. The maximum deflections of all specimens with the exception of PS 5 corresponded to the ultimate strain of the tendons. All specimens dissipated considerable energy as a consequence of the large deflections.

- The ultimate loads of the square plate $P 1$ and the simply supported beam PS 3 were roughly equal to the theoretical ultimate loads of similar specimens with bonded prestressed reinforcement. The ultimate loads of the other platestrips were about two to three and a half times higher than the theoretical ultimate loads of bonded simply supported strips.

- In all tests the tendon forces increased continuously up to the tensile strength.

- The concrete strain distribution depended much on the stiffness of the restraint against lateral movements and on the supplementary bonded reinforcement. After crushing of the outermost concrete layer of the simply supported beam PS 3 the individual parts of the platestrip rotated without noticeable loss of strength until the tendon ruptured somewhat similar to the rotation of two bone ends.

- Cracks always opened continuously, never abruptly. Lateral restraint and additional bonded reinforcement had a marked influence upon the crack pattern and the crack width. 
- In design two limit states must be observed: limit state of serviceability and limit state of failure. Sufficient safety against failure guarantees generally a normal behavior relative to cracks and deformations for systems with bonded reinforcement under working loads. For systems without bond however the limit state of serviceability often governs. 
Der vorliegende Bericht wurde im Rahmen des Forschungsprojektes "Vorgespannte Platten" des Institutes für Baustatik und Konstruktion an der Eidgenässischen Technischen Hochschule in Zürich (ETHZ) ausgearbeitet. Für die grosszügige finanzielle Unterstützung dieses Projektes möchten die Verfasser folgenden Stellen aufrichtig danken:

- Stiftung für wissenschaftliche, systematische Forschungen auf dem Gebiete des Beton- und Eisenbetonbaues, Zürich

- Kommission zur Förderung der wissenschaftlichen Forschung, Bern

- Firma Losinger AG, Bern

- Firma Stahlton.AG, Zürich.

Das Forschungsprojekt wird von einer beratenden Kommission begleitet, die wie folgt zusammengesetzt ist: Dr. M. Birkenmaier, Prof. R. Favre, Dr. M. Ladner, P. Matt, dipl. Ing., K. Messerli, dipl. Ing., R. Weiss, dipl. Ing., Dr. R. Wolfensberger. Diesen Herren sei ihr Mitwirken auch an dieser Stelle bestens verdankt. Der experimentelle Teil der Untersuchungen wurde an der Eidgenössischen Materialprüfungs- und Versuchsanstalt (EMPA) in Dübendorf durchgeführt. Die Verfasser möchten Herrn Prof. Dr. Th. Erismann, Direktionspräsident der EMPA, Herrn Dr. M. Ladner, Vorsteher der Abteilung Massivbau sowie den übrigen an der Versuchsdurchführung beteiligten Mitarbeitern der EMPA für ihr Entgegenkommen bestens danken. Die photogrammetrischen Messungen beim Versuch $P 1$ wurden von Mitarbeitern des Institutes für Geodäsie und Photogrammetrie der Eidgenössischen Technischen Hochschule, Zürich, vorgenommen und ausgewertet. Für die wertvolle und stets angenehme Zusammenarbeit danken wir den Herren Prof. Or. H. Schmid, S. Heggli, dipl. Ing. und J. Rady, dipl. Ing.

Bei der Versuchsdurchführung und der Gestaltung dieses Berichtes haben mitgearbeitet: Frl. S. Burki, die Herren K. Bucher, G. Göseli, M. Rossi, L. Sieger und C. Tiniç. Herr Baumann, dipl.Ing., hat die messtechnischen Probleme bearbeitet. Für ihre Mitarbeit sei den Genannten bestens gedankt. 
[1] ACI-ASCE Committee 423, "Tentative Recommendations for Prestressed Concrete Flat Plates", ACI Journal, February 1974.

[2] Knöpfel, Hans, "Berechnung starr-plastischer Platten mittels finiter Elemente", Institut für Baustatik ETH Zürich, Bericht Nr. 47. August 1973. 


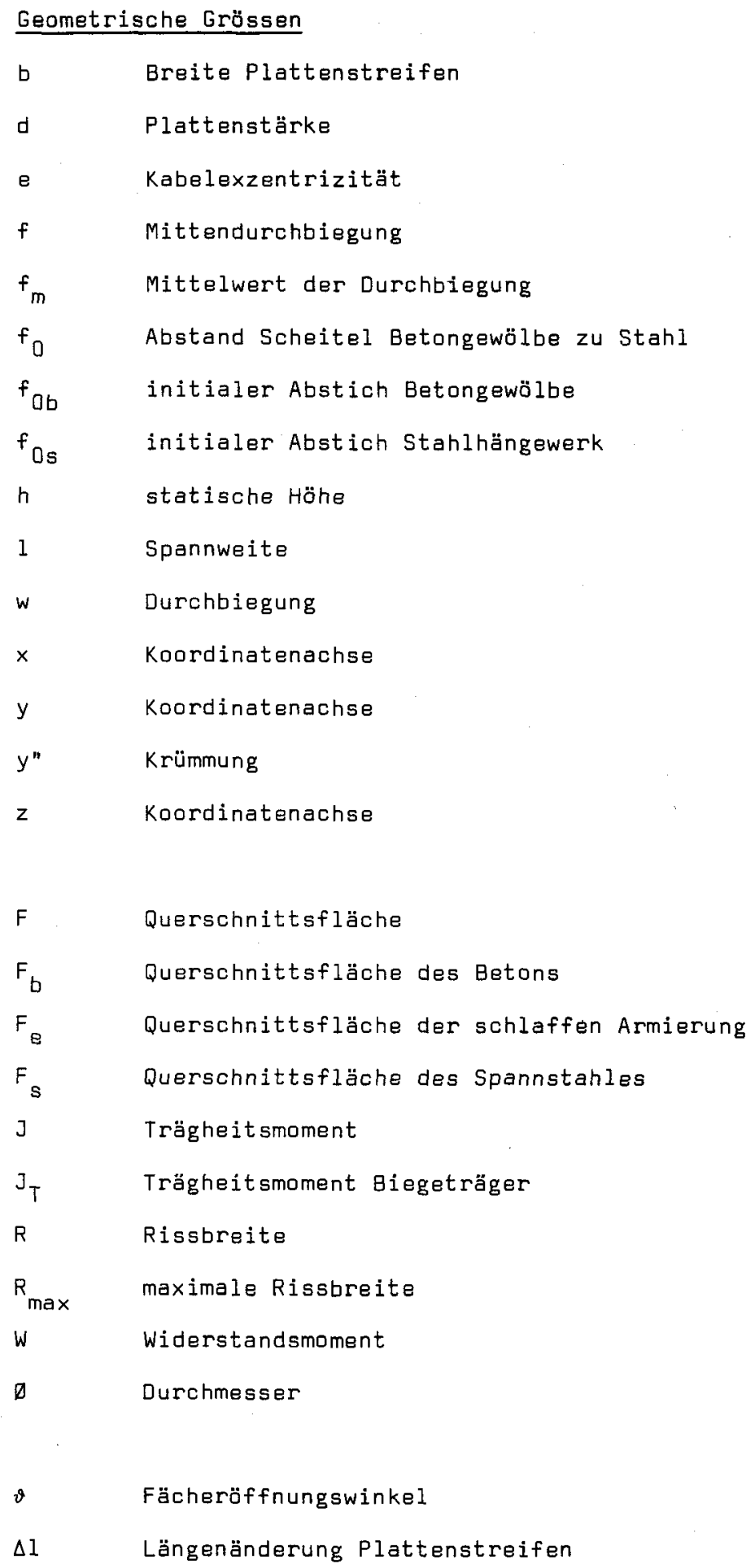




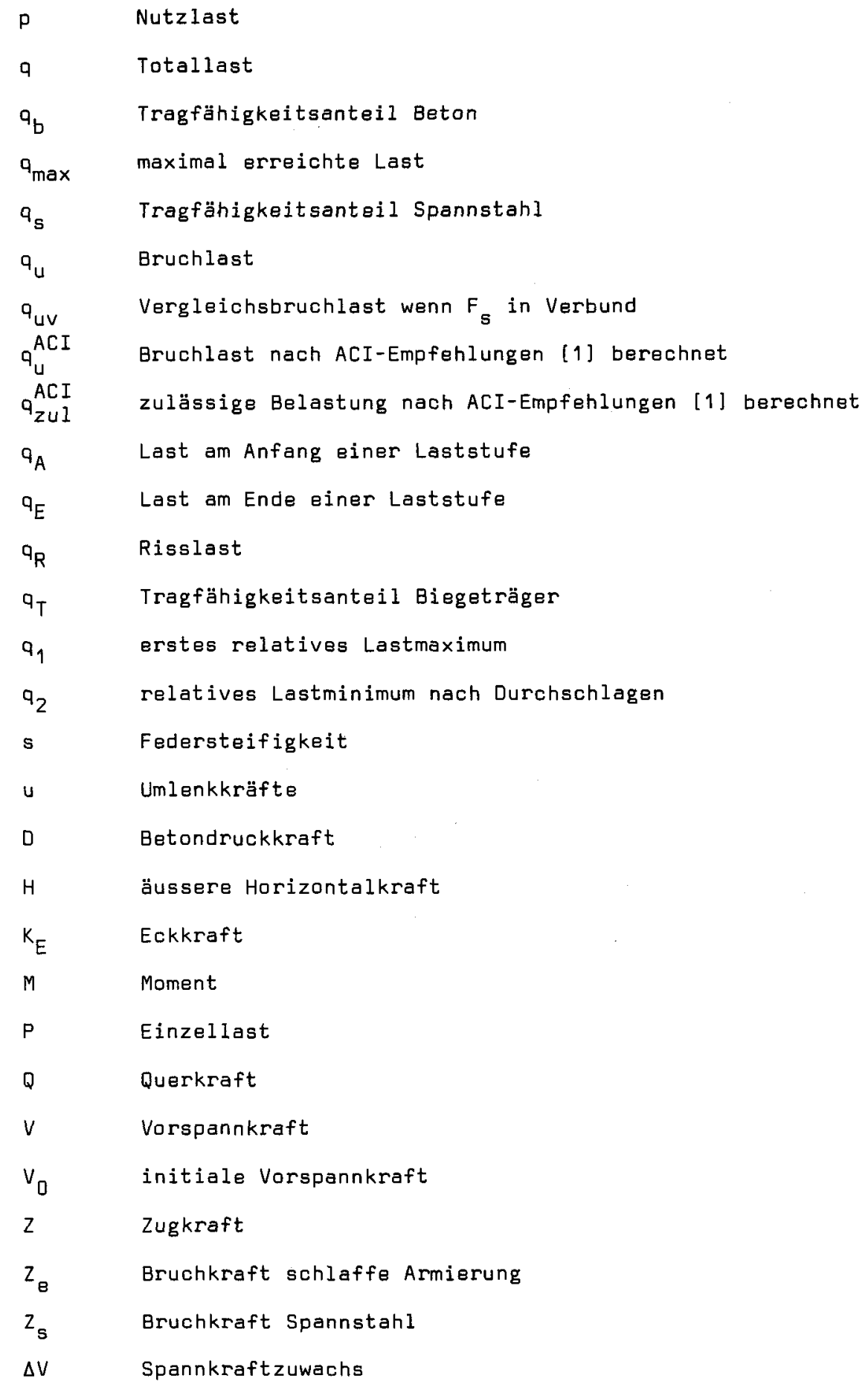

\section{Festigkeitswerte, Spannungen}

$\begin{array}{ll}E_{b} & \text { Elastizitätsmodul Beton } \\ E_{b} 5 \div 50 & \text { Elastizitätsmodul Beton für } \sigma_{b}=5 \div 50 \mathrm{~kg} / \mathrm{cm}^{2} \\ E_{b} 5 \div 100 & \text { Elastizitätsmodul Beton für } \sigma_{b}=5 \div 100 \mathrm{~kg} / \mathrm{cm}^{2} \\ E_{B} & \text { Elastizitätsmodul schlaffe Armierung } \\ E_{s} & \text { Elastizitätsmodul Spannstahl } \\ E_{T} & \text { Elastizitätsmodul Biegeträger } \\ B_{b z} & \text { Biegezugfestigkeit }\end{array}$




$\begin{array}{ll}\beta_{p} & \text { Prismendruckfestigkeit } \\ \beta_{w} & \text { Würfeldruckfestigkeit } \\ \beta_{w 28} & \text { Würfeldruckfestigkeit nach } 28 \text { Tagen } \\ \beta_{z e} & \text { Bruchspannung schlaffe Armierung } \\ \beta_{z s} & \text { Bruchspannung Spannstahl } \\ \sigma_{b} & \text { Betonspannung } \\ \sigma_{b u} & \text { Betonspannung am unteren Rand } \\ \sigma_{b V} & \text { zentrische Vorspannung } \\ \sigma_{e} & \text { Spannung in schlaffer Armierung } \\ \sigma_{e} 0.2 & \text { Streckgrenze schlaffe Armierung } \\ \sigma_{s} & \text { Spannung im Spannstahl } \\ \sigma_{s 0} & \text { Spannung im Spannstahl bei V } \\ \sigma_{s} 0.2 & \text { Streckgrenze Spannstahl }\end{array}$

\section{Verformungen, Zeitgrössen}

$\begin{array}{ll}\varepsilon & \text { Dehnung } \\ \varepsilon_{b} & \text { Betonstauchung } \\ \varepsilon_{e} & \text { Dehnung in schlaffer Armierung } \\ \varepsilon_{s} & \text { Dehnung im Spannstahl } \\ \varepsilon_{0} & \text { Dehnung im Spannstahl bei } V_{0} \\ \dot{\varepsilon} & =d \varepsilon / d t, \text { Dehnungsgeschwindigkeit } \\ \lambda_{G l} & \text { Bruchdehnung über ganze Litzenlänge } \\ t & \text { Zeit }\end{array}$

\section{Dimensionslose Parameter}

$\begin{array}{ll}\gamma & =1-\left(m_{p 1} / m_{p 0}\right) \\ \xi & =x / 1 \\ \mu_{e} & \text { Armierungsgehalt schlaffe Armierung } \\ \mu_{s} & \text { Armierungsgehalt Spannstahl } \\ \nu & \text { Querdehnungszahl }\end{array}$

\section{Allgemeine Bezeichnungen}

$\begin{array}{ll}\text { LS } & \text { Laststufe } \\ \text { P } & \text { Platte } \\ \text { PS } & \text { Plattenstreifen } \\ \text { " } & \text { Zoll (inch) }\end{array}$


Indices

$\begin{array}{ll}\text { b } & \text { Beton } \\ \text { e } & \text { schlaffe Armierung } \\ \text { o } & \text { oben } \\ \text { p } & \text { plastisch } \\ \text { s } & \text { Spannstahl } \\ \text { U } & \text { ultimate, unten } \\ V & \text { Verbund } \\ \text { A } & \text { Anfang einer Laststufe } \\ \text { E } & \text { Ende einer Laststufe } \\ \text { Ex } & \text { experimentell } \\ R & \text { Riss } \\ T & \text { Biegeträger } \\ \text { Th } & \text { theoretisch } \\ V & \text { Vorspannung } \\ \text { D } & \text { initialer Zustand }\end{array}$




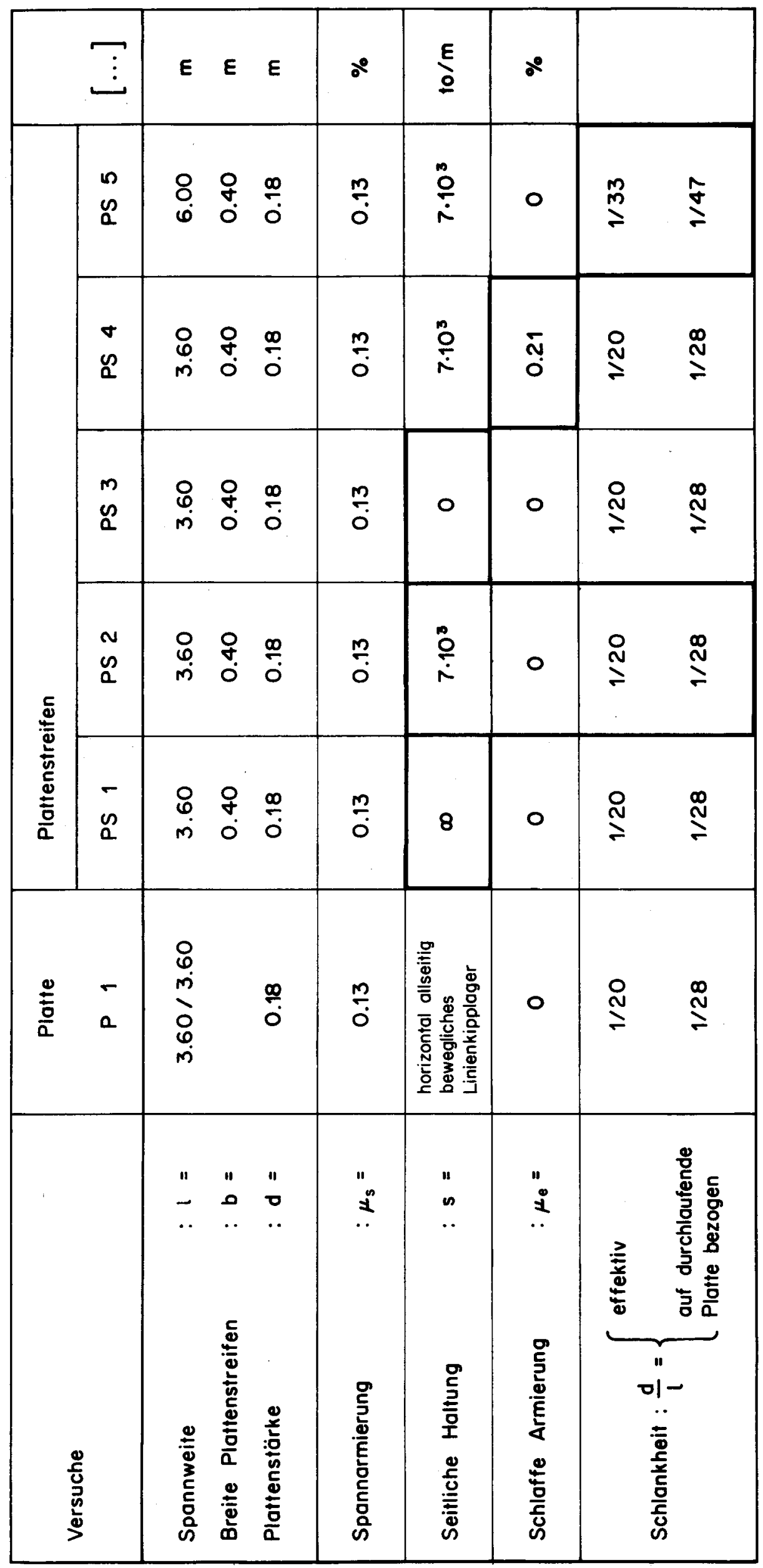

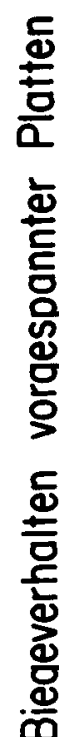

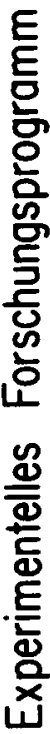

$\because$
$\stackrel{0}{ \pm}$
$\stackrel{0}{\circ}$ 


\begin{tabular}{|c|c|c|c|c|c|c|c|c|}
\hline \multirow{5}{*}{ 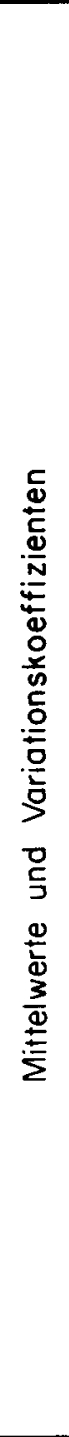 } & 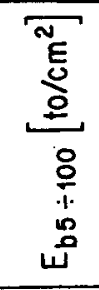 & 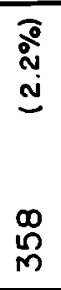 & 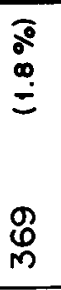 & 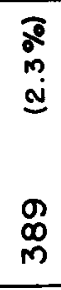 & 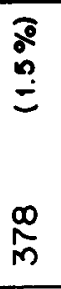 & 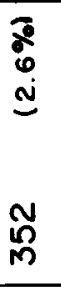 & $\begin{array}{c}\overline{0} \\
\stackrel{0}{+} \\
\bar{\infty} \\
m\end{array}$ & \multirow{3}{*}{ 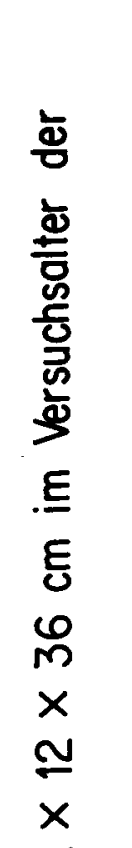 } \\
\hline & 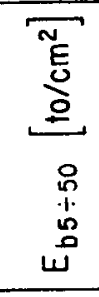 & 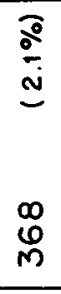 & 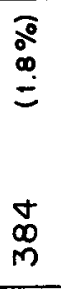 & 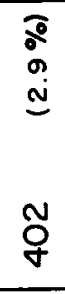 & $\begin{array}{l}\stackrel{0}{0} \\
\infty \\
\stackrel{\infty}{=} \\
0 \\
\infty \\
0 \\
m\end{array}$ & $\begin{array}{l}\text { o. } \\
m \\
m \\
0 \\
0 \\
0 \\
0 \\
m\end{array}$ & \begin{tabular}{l}
$\overline{0}$ \\
$\vdots$ \\
\pm \\
\multirow{్}{*}{}
\end{tabular} & \\
\hline & 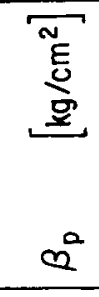 & $\begin{array}{l}\bar{o} \\
\stackrel{0}{ \pm} \\
\stackrel{0}{m} \\
m\end{array}$ & $\begin{array}{l}\frac{\bar{o}}{\bar{m}} \\
\stackrel{\dot{m}}{m}\end{array}$ & 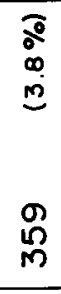 & $\begin{array}{l}\bar{o} \\
\dot{0} \\
\dot{0} \\
\hat{N} \\
\hat{m}\end{array}$ & 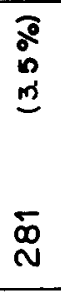 & $\begin{array}{l}\text { o } \\
0 \\
\dot{0} \\
\vdots \\
8\end{array}$ & \\
\hline & 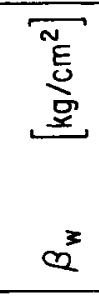 & $\begin{array}{l}\bar{o} \\
\stackrel{0}{m} \\
\dot{0} \\
\stackrel{\sigma}{\sigma}\end{array}$ & 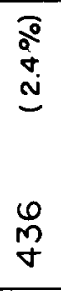 & $\begin{array}{l}\dot{q} \\
\dot{0} \\
\dot{0} \\
\dot{0} \\
\dot{0}\end{array}$ & 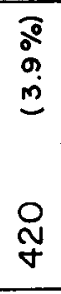 & $\begin{array}{l}\frac{8}{\vdots} \\
\vdots \\
\stackrel{2}{m}\end{array}$ & $\begin{array}{l}\widehat{\Im} \\
0 \\
0 \\
\text { త్}\end{array}$ & 등 \\
\hline & 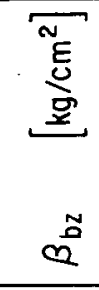 & $\begin{array}{l}\overline{0} \\
i n \\
0 \\
0\end{array}$ & $\begin{array}{l}\frac{0}{\circ} \\
\stackrel{0}{\circ} \\
\stackrel{m}{+}\end{array}$ & $\begin{array}{l}\text { o. } \\
\dot{0} \\
\dot{0}\end{array}$ & $\begin{array}{l}5 \\
0 \\
0 \\
0 \\
\end{array}$ & $\begin{array}{l}\text { o } \\
\circ \\
0\end{array}$ & 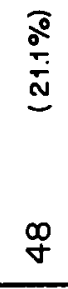 & 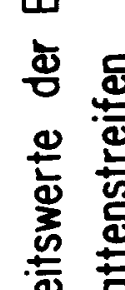 \\
\hline 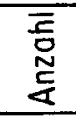 & $\begin{array}{l}\bar{\Phi} \\
\text { 옹 } \\
\end{array}$ & $\sigma$ & $\nabla$ & $\theta$ & $\sigma$ & $\sigma$ & $\sigma$ & 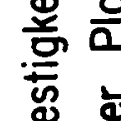 \\
\hline$\frac{\grave{\Phi}}{\frac{\Phi}{4}}$ & $\stackrel{\stackrel{\mathscr{g}}{\circ}}{\stackrel{0}{0}}$ & $\stackrel{m}{m}$ & $\stackrel{\infty}{\sim}$ & $\mathscr{m}$ & $\stackrel{\mathscr{n}}{m}$ & $\stackrel{n}{m}$ & $\stackrel{m}{m}$ & $\begin{array}{l}\Psi \\
\ddot{\sigma} \\
\dot{\sigma}\end{array}$ \\
\hline & & - & $\begin{array}{l}\sigma \\
\tilde{a}\end{array}$ & $\begin{array}{l}N \\
\infty \\
a\end{array}$ & $\begin{array}{l}m \\
n \\
0\end{array}$ & $\begin{array}{l}\sigma \\
a \\
a\end{array}$ & $\begin{array}{l}n \\
0 \\
a\end{array}$ & 응 \\
\hline
\end{tabular}
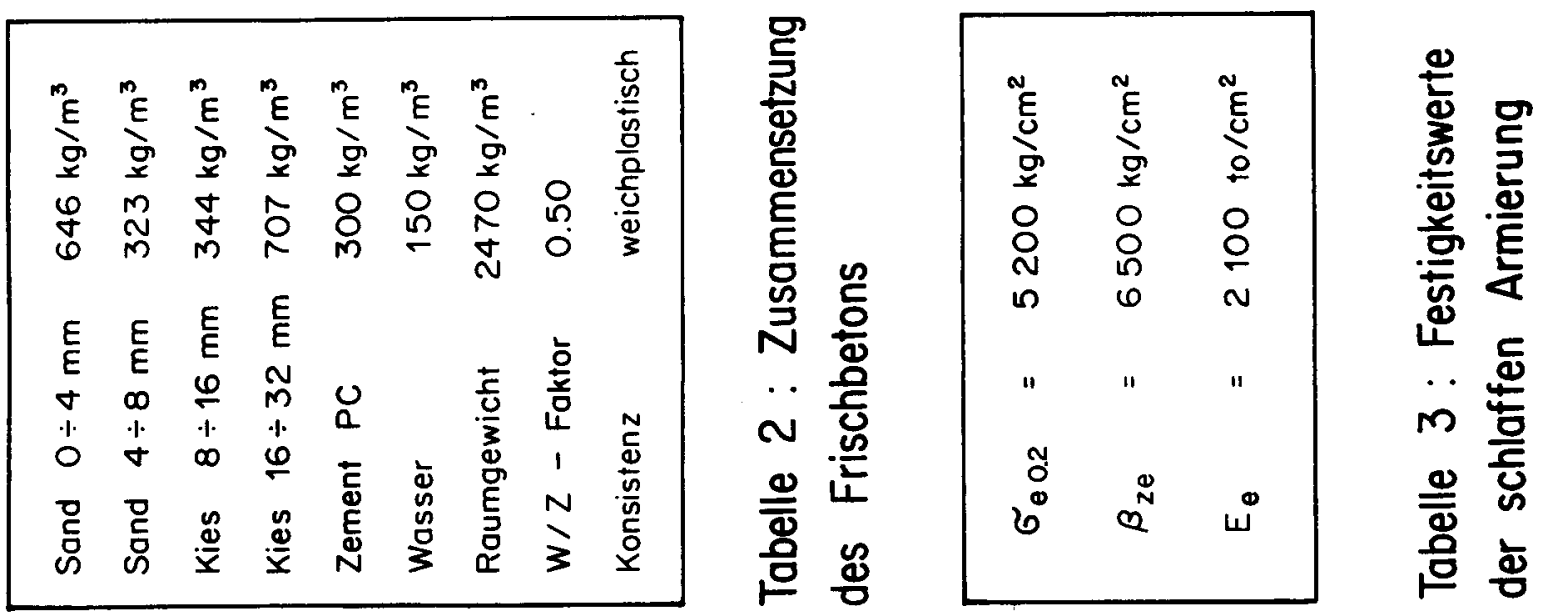


\begin{tabular}{|c|c|c|c|c|}
\hline \multirow{2}{*}{ LS } & $f$ & $q_{A}$ & $q_{E}$ & $K_{E}$ \\
\hline & {$[\mathrm{cm}]$} & {$\left[10 / m^{2}\right]$} & {$\left[10 / \mathrm{m}^{2}\right]$} & [10] \\
\hline 1 & 0.06 & 2.12 & 2.02 & 1.0 \\
\hline 2 & 0.17 & 4.01 & 3.80 & 1.0 \\
\hline 3 & 0.30 & 6.01 & 5.79 & 2.8 \\
\hline 4 & 0.94 & 7.79 & 7.48 & 2.8 \\
\hline 5 & 0.02 & 0.45 & 0.45 & 0 \\
\hline 6 & 0.13 & 2.12 & 2.06 & 1.0 \\
\hline 7 & 0.27 & 4.01 & 3.91 & 1.0 \\
\hline 8 & 0.53 & 6.01 & 5.91 & 28 \\
\hline 9 & 1.32 & 8.00 & 7.71 & 2.8 \\
\hline 10 & 0.05 & 0.45 & 0.45 & 0 \\
\hline 11 & 0.14 & 2.12 & 2.07 & 1.0 \\
\hline 12 & 0.31 & 4.01 & 3.93 & 1.0 \\
\hline 13 & 0.35 & 4.49 & 4.38 & 2.8 \\
\hline 14 & 0.41 & 4.99 & 4.87 & 2.8 \\
\hline 15 & 0.50 & 5.50 & 5.44 & 2.8 \\
\hline 16 & 0.62 & 6.00 & 5.89 & 2.8 \\
\hline 17 & 0.05 & 0.45 & 0.45 & 0 \\
\hline 18 & 0.05 & 0.45 & 0.45 & 0 \\
\hline 19 & 0.15 & 2.12 & 2.09 & 1.0 \\
\hline 20 & 0.31 & 4.01 & 3.97 & 1.0 \\
\hline 21 & 0.64 & 6.00 & 5.93 & 2.8 \\
\hline 22 & 0.81 & 6.50 & 6.36 & 2.8 \\
\hline 23 & 1.01 & 6.99 & 6.87 & 2.8 \\
\hline 24 & 1.25 & 7.49 & 7.38 & 3.3 \\
\hline 25 & 1.53 & 8.00 & 7.85 & 3.3 \\
\hline 26 & 2.10 & 8.50 & 8.16 & 4.8 \\
\hline 27 & 3.10 & 8.98 & 8.63 & 4.8 \\
\hline 28 & 4.10 & 9.34 & 9.01 & 4.8 \\
\hline 29 & 5.14 & 9.67 & 9.30 & 4.8 \\
\hline 30 & 6.14 & 9.92 & 9.49 & 5.3 \\
\hline 31 & 7.14 & 10.06 & 9.53 & 5.3 \\
\hline 32 & 8.14 & 10.20 & 9.55 & 5.3 \\
\hline 33 & 9.14 & 10.20 & 9.51 & 5.3 \\
\hline 34 & 11.14 & 10.16 & 9.18 & 5.9 \\
\hline 35 & 16.14 & 10.16 & 9.67 & - \\
\hline 36 & 16.14 & 9.61 & 9.40 & - \\
\hline 37 & 21.14 & 10.43 & 9.67 & - \\
\hline 38 & 26.14 & 10.40 & 9.30 & - \\
\hline 39 & 26.75 & 9.26 & $\longrightarrow$ & - \\
\hline
\end{tabular}

Tabelle 5: Belastungsablauf Platte P1 


\begin{tabular}{|c|c|c|c|c|c|}
\hline \multirow{2}{*}{ LS } & $f$ & $q_{A}$ & $a_{E}$ & H & $\Delta V$ \\
\hline & {$[\mathrm{cm}]$} & {$[\mathrm{to} / \mathrm{m}]$} & {$[10 / m]$} & {$[10]$} & {$[.10]$} \\
\hline 1 & 0 & 0.18 & 0.18 & 0 & 0 \\
\hline 2 & 0.10 & 0.53 & 0.49 & -1.20 & 0 \\
\hline 3 & 0.29 & 1.02 & 0.94 & -1.20 & 0.02 \\
\hline 4 & 0.77 & 1.37 & 1.25 & -9.50 & 0.33 \\
\hline 5 & 1.36 & 1.57 & 1.45 & -15.00 & 0.71 \\
\hline 6 & 0.12 & 0.18 & 0.18 & -0.86 & 0.12 \\
\hline 7 & 0.38 & 0.74 & 0.69 & -1.30 & 0.12 \\
\hline 8 & 1.22 & 1.37 & 1.31 & -12.05 & 0.51 \\
\hline 9 & 1.69 & 1.59 & 1.49 & -17.70 & 0.97 \\
\hline 10 & 0.15 & 0.18 & 0.18 & 0 & 0.13 \\
\hline 11 & 0.44 & 0.67 & 0.64 & -1.30 & 0.22 \\
\hline 12 & 0.66 & 0.89 & 0.87 & -3.85 & 0.24 \\
\hline 13 & 0.72 & 0.94 & 0.90 & -4.80 & 0.26 \\
\hline 14 & 0.83 & 1.01 & 0.98 & -5.95 & 0.31 \\
\hline 15 & 1.01 & 1.14 & 1.11 & -8.45 & 0.42 \\
\hline 16 & 1.24 & 1.28 & 1.25 & -11.00 & 0.57 \\
\hline 17 & 0.14 & 0.18 & 0.18 & 0 & 0.12 \\
\hline 18 & 0.11 & 0.18 & 0.18 & 0 & 0.10 \\
\hline 19 & 1.37 & 1.37 & 1.31 & -13.40 & 0.65 \\
\hline 20 & 1.59 & 1.48 & 1.44 & -16.10 & 0.86 \\
\hline 21 & 2.15 & 1.57 & 1.54 & -21.50 & 1.33 \\
\hline 22 & 2.85 & 1.62 & 1.50 & -23.70 & 1.87 \\
\hline 23 & 3.51 & 1.55 & 1.47 & -26.00 & 2.33 \\
\hline 24 & 4.53 & 1.45 & 1.40 & -27.20 & 2.89 \\
\hline 25 & 5.54 & 1.25 & 1.18 & -24.70 & 3.36 \\
\hline 26 & 0.55 & 0.18 & 0.18 & -1.90 & 0.28 \\
\hline 260 & 5.51 & 1.20 & 1.16 & -23.50 & 3.22 \\
\hline 27 & 6.51 & 1.09 & 1.02 & -19.90 & 3.78 \\
\hline 28 & 9.01 & 1.21 & 1.15 & -4.40 & 4.64 \\
\hline 29 & 13.94 & 2.25 & 2.15 & +15.50 & 5.73 \\
\hline 30 & 18.94 & 3.25 & 3.14 & +20.90 & 6.13 \\
\hline 31 & 18.54 & 2.67 & 2.62 & +16.30 & 5.11 \\
\hline 32 & 23.50 & 3.94 & 3.60 & +19.40 & 6.33 \\
\hline 33 & 24.59 & 4.05 & - & $\longrightarrow$ & 6.57 \\
\hline
\end{tabular}

Tabelle 6: Belastungsablauf Plattenstreifen PS 1 


\begin{tabular}{|c|c|c|c|c|c|}
\hline \multirow{2}{*}{ LS } & $f$ & $q_{A}$ & $q_{E}$ & $\mathrm{H}$ & $\Delta V$ \\
\hline & {$[\mathrm{cm}]$} & {$[10 / \mathrm{m}]$} & {$[10 / \mathrm{m}]$} & [10] & [to] \\
\hline 1 & 0 & 0.18 & 0.18 & 0 & 0 \\
\hline 2 & 0.12 & 0.53 & 0.49 & -2.00 & 0.02 \\
\hline 3 & 0.74 & 1.09 & 1.12 & -2.40 & 0.50 \\
\hline 4 & 1.23 & 1.31 & 1.29 & -4.30 & 0.82 \\
\hline 5 & 1.72 & 1.40 & 1.35 & -6.30 & 1.13 \\
\hline 6 & 0.11 & 0.18 & 0.18 & 0 & 0.16 \\
\hline 7 & 0.44 & 0.82 & 0.77 & -1.90 & 0.19 \\
\hline 8 & 0.54 & 0.91 & 0.87 & -2.10 & 0.23 \\
\hline 9 & 0.71 & 1.01 & 1.02 & -2.20 & 0.33 \\
\hline 10 & 0.93 & 1.14 & 1.11 & -3.40 & 0.49 \\
\hline 11 & 1.22 & 1.24 & 1.20 & -4.50 & 0.73 \\
\hline 12 & 1.72 & 1.40 & 1.37 & -6.30 & 1.13 \\
\hline 13 & 2.21 & 1.47 & 1.45 & -7.75 & 1.42 \\
\hline 14 & 3.20 & 1.55 & 1.51 & -10.50 & 1.99 \\
\hline 15 & 4.20 & 1.51 & 1.49 & -12.20 & 2.47 \\
\hline 16 & 5.20 & 1.38 & 1.34 & -13.00 & 2.96 \\
\hline 17 & 6.20 & 1.29 & 1.25 & -12.60 & 3.31 \\
\hline 18 & 7.70 & 1.20 & 1.17 & -9.40 & 3.75 \\
\hline 19 & 9.19 & 1.18 & 1.16 & -6.50 & 4.07 \\
\hline 20 & 0.97 & 0.18 & 0.18 & 0 & -2.22 \\
\hline 21 & 0.82 & 0.18 & 0.18 & 0 & -2.29 \\
\hline 22 & 9.18 & 1.18 & 1.12 & -4.00 & 3.84 \\
\hline 23 & 10.15 & 1.31 & 1.29 & -1.00 & 4.31 \\
\hline 24 & 12.12 & 1.58 & 1.51 & +3.80 & 4.50 \\
\hline 25 & 15.09 & 2.05 & 1.97 & +11.00 & 4.72 \\
\hline 26 & 18.14 & 2.79 & 2.75 & +18.30 & 4.82 \\
\hline 27 & 18.14 & 2.51 & 2.48 & +14.80 & 4.77 \\
\hline 28 & 22.13 & 3.62 & 3.44 & +20.70 & 5.13 \\
\hline 29 & 25.26 & 3.89 & - & +19.52 & 5.45 \\
\hline
\end{tabular}

Tabelle 7: Belastungsablauf Plattenstreifen PS 2 


\begin{tabular}{|c|c|c|c|c|c|}
\hline \multirow{2}{*}{ LS } & $f$ & $q_{A}$ & $q_{E}$ & $\Delta \mathrm{l}$ & $\Delta V$ \\
\hline & {$[\mathrm{cm}]$} & {$[\mathrm{to} / \mathrm{m}]$} & {$[\mathrm{to} / \mathrm{m}]$} & {$[\mathrm{mm}]$} & {$[10]$} \\
\hline 1 & 0 & 0.18 & 0.18 & 0 & 0 \\
\hline 2 & 0.20 & 0.74 & 0.71 & 0 & 0 \\
\hline 3 & 0.39 & 1.14 & 1.08 & 0 & 0.08 \\
\hline 4 & 0.48 & 1.20 & 1.05 & 0.03 & 0.20 \\
\hline 5 & 1.06 & 1.19 & 1.11 & 0.33 & 0.69 \\
\hline 6 & 1.64 & 1.25 & 1.16 & 0.59 & 1.17 \\
\hline 7 & 0.11 & 0.18 & 0.18 & 0.06 & 0.08 \\
\hline 8 & 0.32 & 0.74 & 0.71 & 0.04 & 0.09 \\
\hline 9 & 0.40 & 0.86 & 0.85 & 0.05 & 0.11 \\
\hline 10 & 0.50 & 0.94 & 0.91 & 0.08 & 0.16 \\
\hline 11 & 0.68 & 1.01 & 0.98 & 0.15 & 0.29 \\
\hline 12 & 0.93 & 1.08 & 1.02 & 0.26 & 0.50 \\
\hline 13 & 1.22 & 1.16 & 1.08 & 0.39 & 0.77 \\
\hline 14 & 1.64 & 1.22 & 1.16 & 0.58 & 1.14 \\
\hline 15 & 2.03 & 1.29 & 1.19 & 0.73 & 1.48 \\
\hline 16 & 2.51 & 1.31 & 1.25 & 0.89 & 1.80 \\
\hline 17 & 3.01 & 1.36 & 1.27 & 1.06 & 2.15 \\
\hline 18 & 3.49 & 1.39 & 1.29 & 1.19 & 2.47 \\
\hline 19 & 4.48 & 1.42 & 1.35 & 1.44 & 2.98 \\
\hline 20 & 5.48 & 1.48 & 1.38 & 1.62 & 3.46 \\
\hline 21 & 6.97 & 1.52 & 1.40 & 1.70 & 3.99 \\
\hline 22 & 8.95 & 1.58 & 1.45 & 1.58 & 4.52 \\
\hline 23 & 11.38 & 1.58 & 1.44 & 0.64 & 4.88 \\
\hline 24 & 16.43 & 1.54 & 1.45 & -4.67 & 5.28 \\
\hline 25 & 17.75 & 1.48 & 1.40 & -7.88 & 5.11 \\
\hline 26 & 21.74 & 1.51 & 1.38 & $\longrightarrow$ & 5.42 \\
\hline 27 & 26.00 & 1.49 & - & - & 5.60 \\
\hline
\end{tabular}

Tabelle 8: Belastungsablouf Plattenstreifen PS 3 


\begin{tabular}{|c|c|c|c|c|c|}
\hline \multirow{2}{*}{ LS } & $f$ & $q_{A}$ & $q_{E}$ & H & $\Delta V$ \\
\hline & {$[\mathrm{cm}]$} & {$[10 / \mathrm{m}]$} & {$[10 / \mathrm{m}]$} & [10] & [10] \\
\hline 1 & 0 & 0.18 & 0.18 & 0 & 0 \\
\hline 2 & 0.11 & 0.53 & 0.51 & -2.00 & 0 \\
\hline 3 & 0.36 & 1.09 & 1.04 & -2.00 & 0.03 \\
\hline 4 & 0.53 & 1.31 & 1.22 & -2.00 & 0.11 \\
\hline 5 & 1.64 & 1.74 & 1.65 & -3.20 & 0.82 \\
\hline 6 & 0.15 & 0.18 & 0.18 & 0 & 0.07 \\
\hline 7 & 0.44 & 0.82 & 0.80 & -0.80 & 0.09 \\
\hline 8 & 0.54 & 0.91 & 0.88 & -0.50 & 0.14 \\
\hline 9 & 0.67 & 1.01 & 0.98 & -0.80 & 0.20 \\
\hline 10 & 0.86 & 1.14 & 1.09 & -1.30 & 0.32 \\
\hline 11 & 1.07 & 1.28 & 1.21 & -1.76 & 0.44 \\
\hline 12 & 1.48 & 1.55 & 1.47 & -2.80 & 0.69 \\
\hline 13 & 1.99 & 1.82 & 1.75 & -3.90 & 1.00 \\
\hline 14 & 3.35 & 2.09 & 1.98 & -7.00 & 1.78 \\
\hline 15 & 4.86 & 2.17 & 2.00 & -9.00 & 2.50 \\
\hline 16 & 5.86 & 2.18 & 1.98 & -9.20 & 2.85 \\
\hline 17 & 7.35 & 2.11 & 1.87 & -8.20 & 3.31 \\
\hline 18 & 9.30 & 2.05 & 1.89 & -3.80 & 3.80 \\
\hline 19 & 10.32 & 2.11 & 1.98 & -0.40 & 3.96 \\
\hline 20 & 2.76 & 0.18 & 0.18 & 0 & -1.02 \\
\hline 21 & 10.29 & 2.11 & 1.98 & 0.33 & 3.76 \\
\hline 22 & 13.23 & 2.62 & 2.45 & 8.53 & 4.45 \\
\hline 23 & 18.16 & 3.90 & 3.69 & 21.65 & 4.53 \\
\hline $23 a$ & 22.93 & 5.25 & - & 27.72 & 5.25 \\
\hline 24 & 23.12 & 3.31 & 3.36 & 15.58 & 4.22 \\
\hline 25 & 24.93 & 4.40 & - & 18.70 & 5.65 \\
\hline
\end{tabular}

Tabelle 9: Belastungsablauf Plattenstreifen PS 4 


\begin{tabular}{|c|c|c|c|c|c|}
\hline \multirow{2}{*}{ LS } & $f$ & $\mathbf{q}_{A}$ & $q_{E}$ & $H$ & $\Delta V$ \\
\hline & {$[\mathrm{cm}]$} & {$[10 / m]$} & {$[10 / m]$} & [10] & [10] \\
\hline 1 & 0 & 0.18 & 0.18 & 0 & 0 \\
\hline 2 & 0.18 & 0.27 & 0.27 & -0.20 & 0 \\
\hline 3 & 0.42 & 0.36 & 0.34 & -0.20 & 0 \\
\hline 4 & 3.31 & 0.49 & 0.43 & -4.24 & 0.72 \\
\hline 5 & 0.50 & 0.18 & 0.18 & -0.20 & 0.22 \\
\hline 6 & 0.65 & 0.27 & 0.26 & -0.66 & 0.21 \\
\hline 7 & 1.60 & 0.39 & 0.37 & -1.60 & 0.27 \\
\hline 8 & 2.58 & 0.44 & 0.41 & -3.00 & 0.50 \\
\hline 9 & 3.54 & 0.47 & 0.44 & -4.20 & 0.76 \\
\hline 10 & 5.03 & 0.49 & 0.43 & -5.60 & 1.22 \\
\hline 11 & 6.54 & 0.49 & 0.44 & -5.86 & 1.63 \\
\hline 12 & 8.04 & 0.47 & 0.43 & -5.00 & 2.06 \\
\hline 13 & 9.51 & 0.46 & 0.44 & -2.90 & 2.46 \\
\hline 14 & 10.85 & 0.49 & 0.47 & 0 & 2.82 \\
\hline 15 & 12.84 & 0.61 & 0.57 & 5.00 & 3.31 \\
\hline 16 & 1.58 & 0.18 & 0.18 & 0 & -0.22 \\
\hline 17 & 12.83 & 0.61 & 0.58 & 5.41 & 3.23 \\
\hline 18 & 17.35 & 0.89 & 0.85 & 13.53 & 4.32 \\
\hline 19 & 22.30 & 1.36 & 1.31 & 20.17 & 5.16 \\
\hline $19 a$ & 26.27 & 1.60 & $\longrightarrow$ & 20.99 & 5.46 \\
\hline 20 & 26.26 & 1.44 & 1.22 & 15.74 & 4.90 \\
\hline 21 & 26.26 & 1.31 & 1.27 & 15.74 & 4.97 \\
\hline $21 a$ & 30.27 & 1.81 & $\longrightarrow$ & 20.99 & 5.84 \\
\hline $21 b$ & 30.77 & 1.58 & - & 17.55 & 5.45 \\
\hline $21 \mathrm{c}$ & 31.27 & 1.77 & - & 20.00 & 5.91 \\
\hline $21 d$ & 34.77 & 2.01 & - & 21.32 & 6.19 \\
\hline
\end{tabular}

Tabelle 10: Belastungsablauf Plattenstreifen PS5 


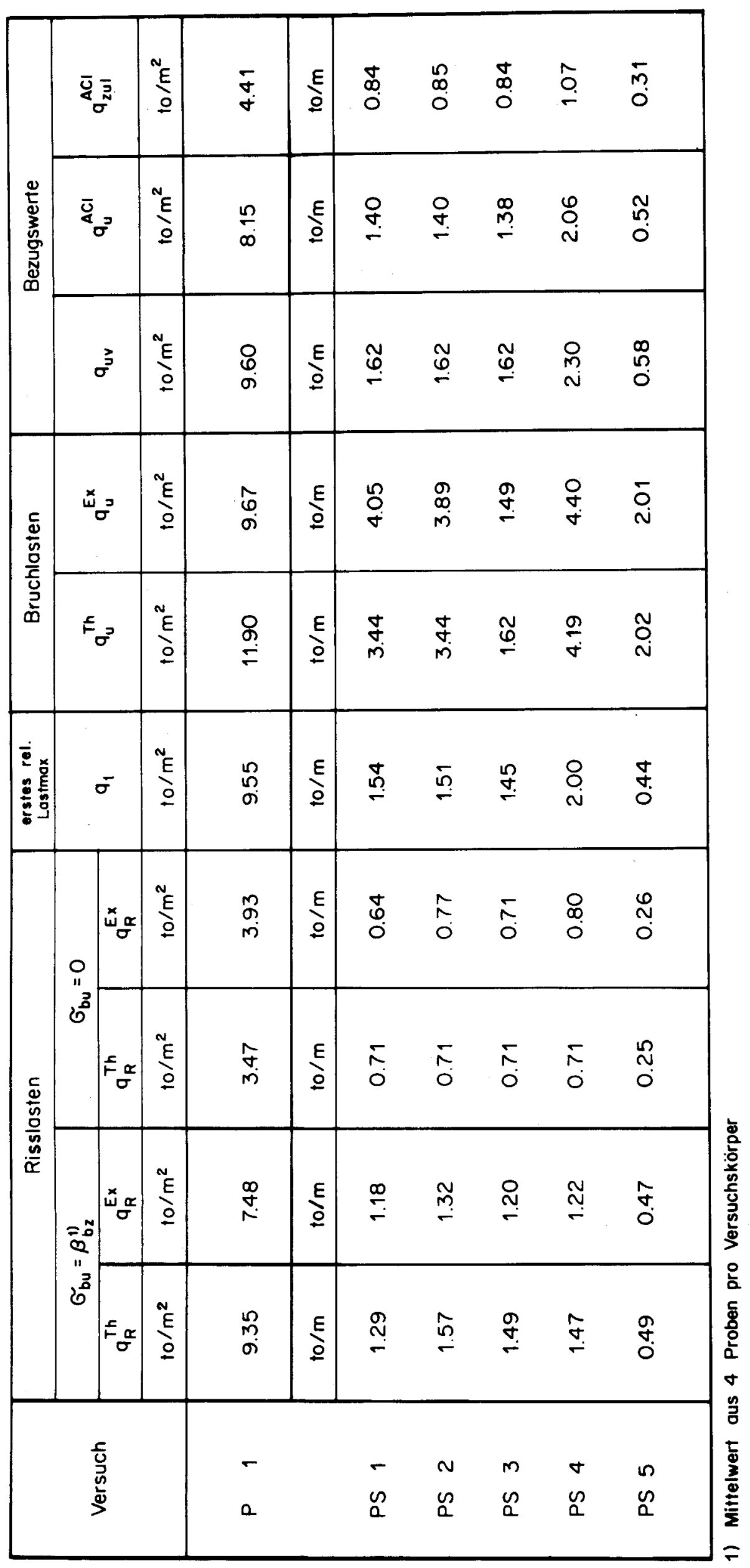

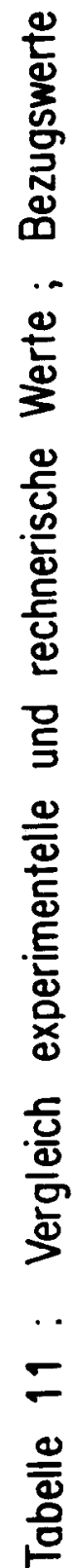




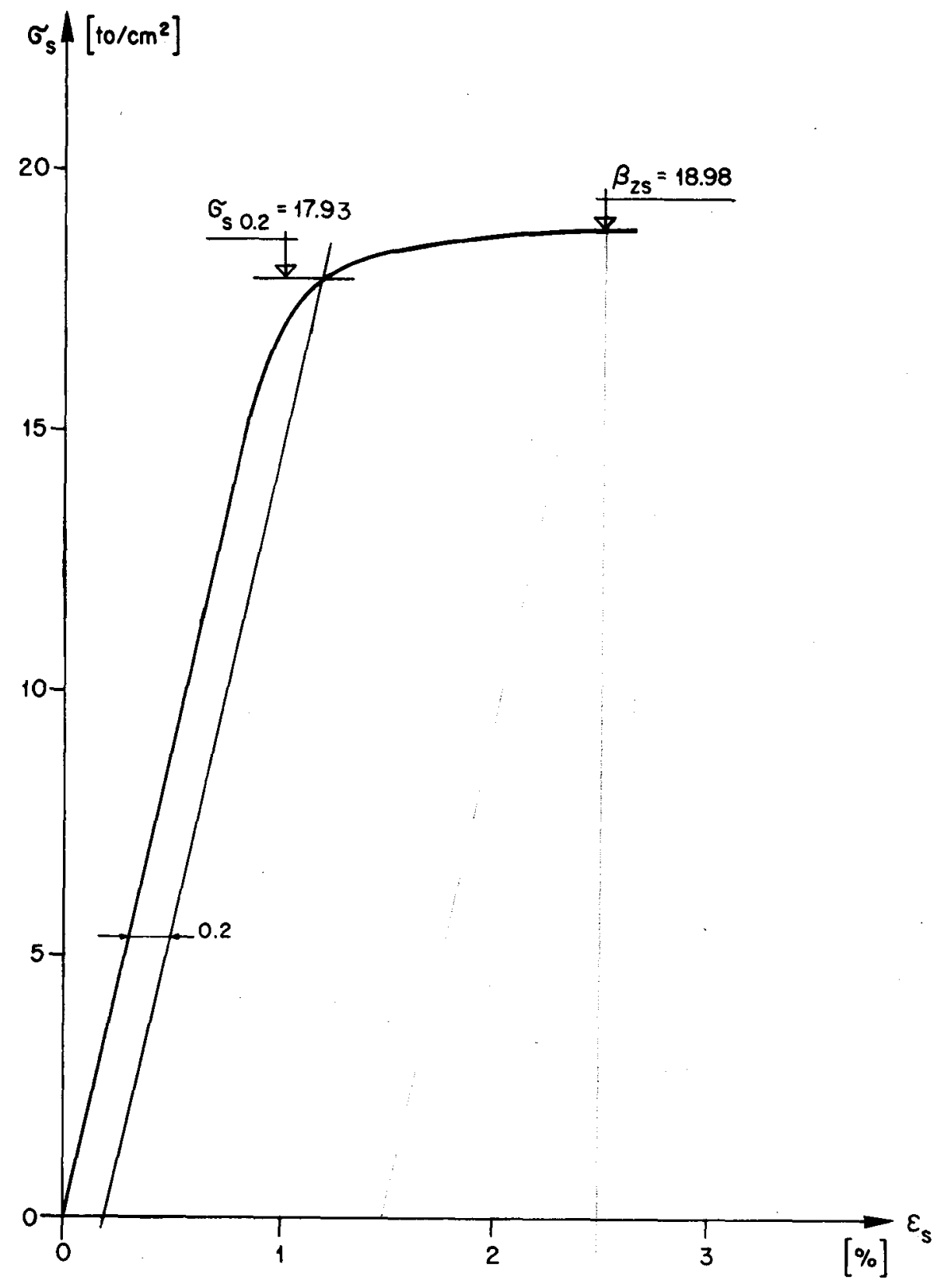

\begin{tabular}{|c|c|c|c|c|c|c|c|c|}
\hline \multirow{2}{*}{$\begin{array}{l}\text { Länge } \\
\text { Litzen }\end{array}$} & \multirow{3}{*}{$\begin{array}{l}\text { Anzahl } \\
\text { Proben }\end{array}$} & \multirow{2}{*}{ Fs } & \multicolumn{2}{|c|}{$\sigma_{s} 0.2$} & \multicolumn{2}{|c|}{$\beta_{\text {zS }}$} & \multirow{2}{*}{$\lambda_{G 1}$} & \multirow{2}{*}{$E$} \\
\hline & & & stotisch & dynomisch & statisch & dynamisch & & \\
\hline $\mathrm{cm}$ & & $\mathrm{cm}^{2}$ & to $/ \mathrm{cm}^{2}$ & $10 / \mathrm{cm}^{2}$ & $10 / \mathrm{cm}^{2}$ & to $/ \mathrm{cm}^{2}$ & $\%$ & to $/ \mathrm{cm}^{2}$ \\
\hline 394 & 2 & 0.93 & 17.03 & 17.93 & 18.03 & 18.98 & 2.66 & 1900 \\
\hline
\end{tabular}

Bild 1: Physikalische Eigenschaften der Vorspannlitzen (Proben) 

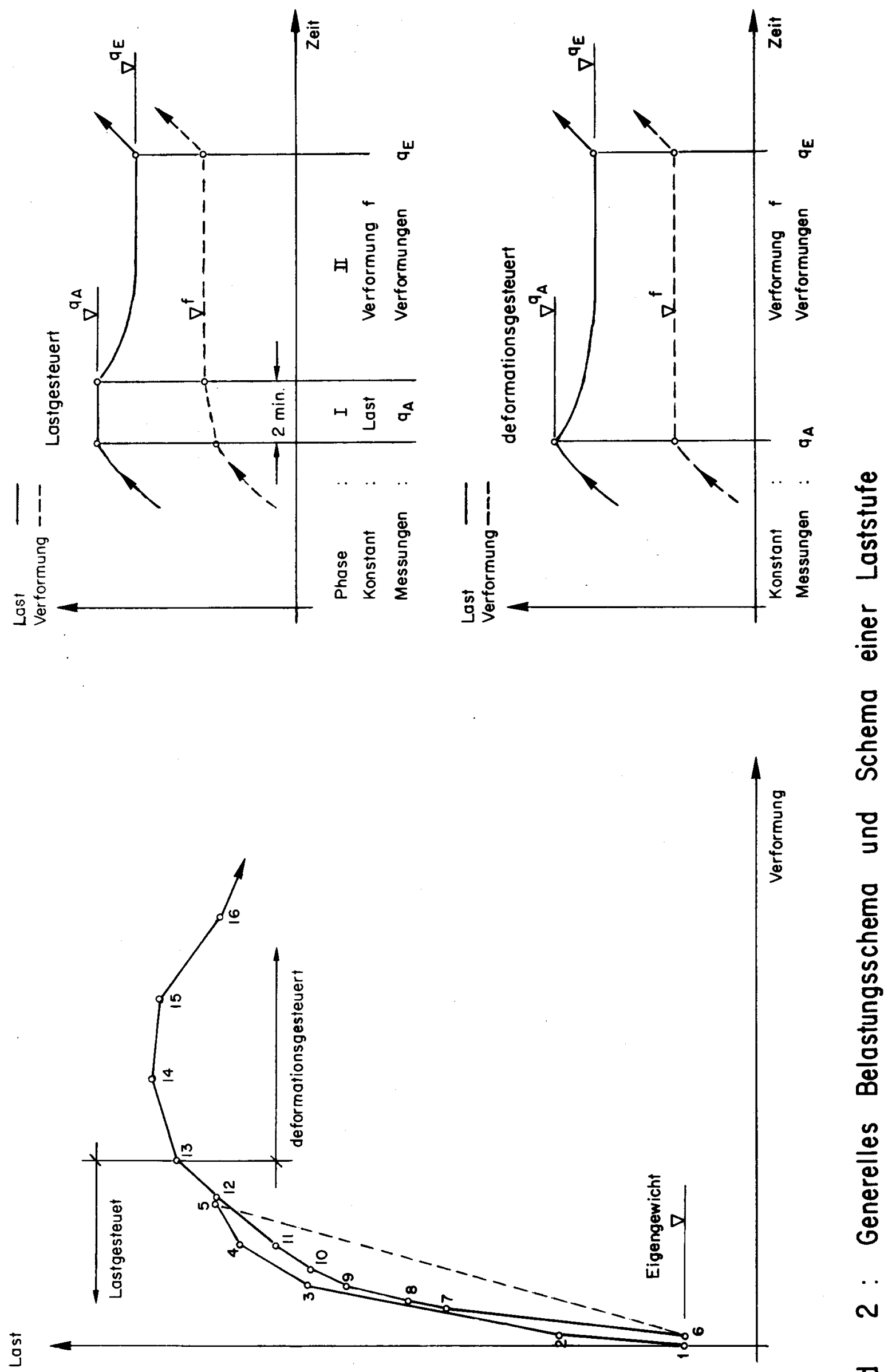

音

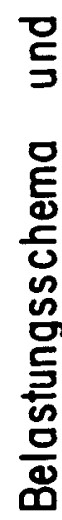

ڤ્)

$\cdots$

흠 

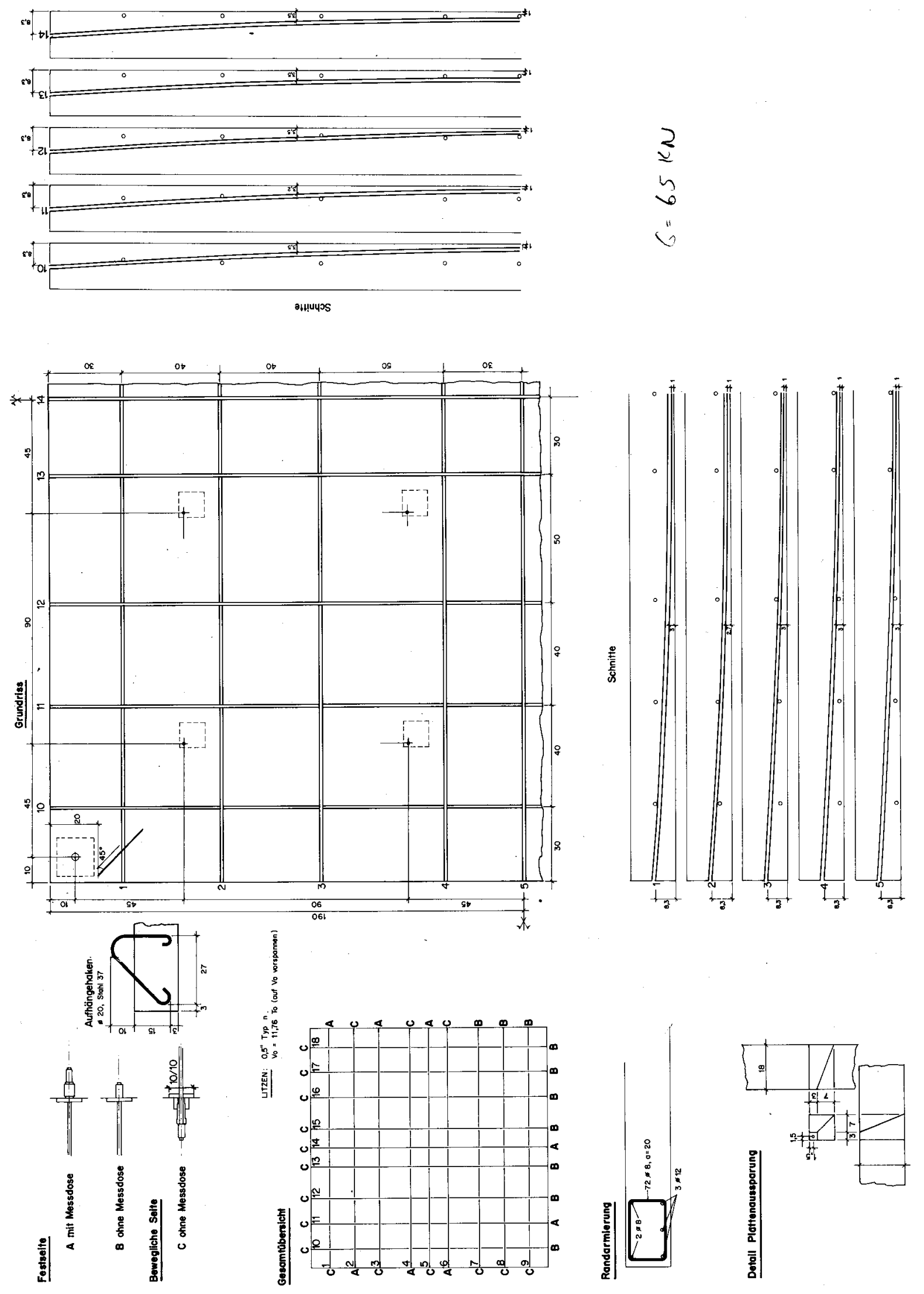

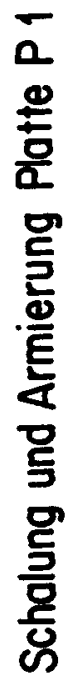
$\dot{m}$ 


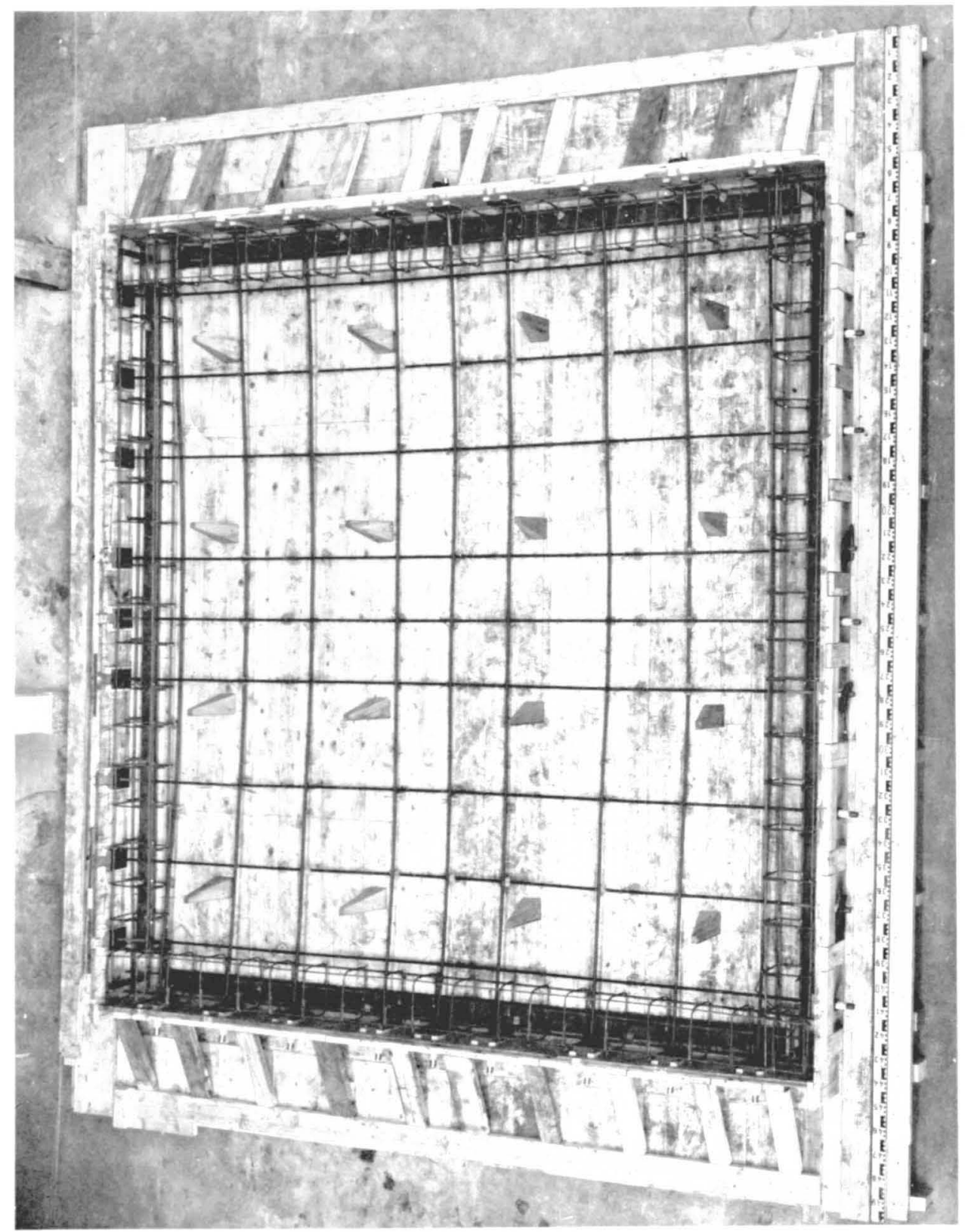

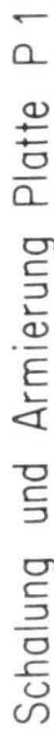



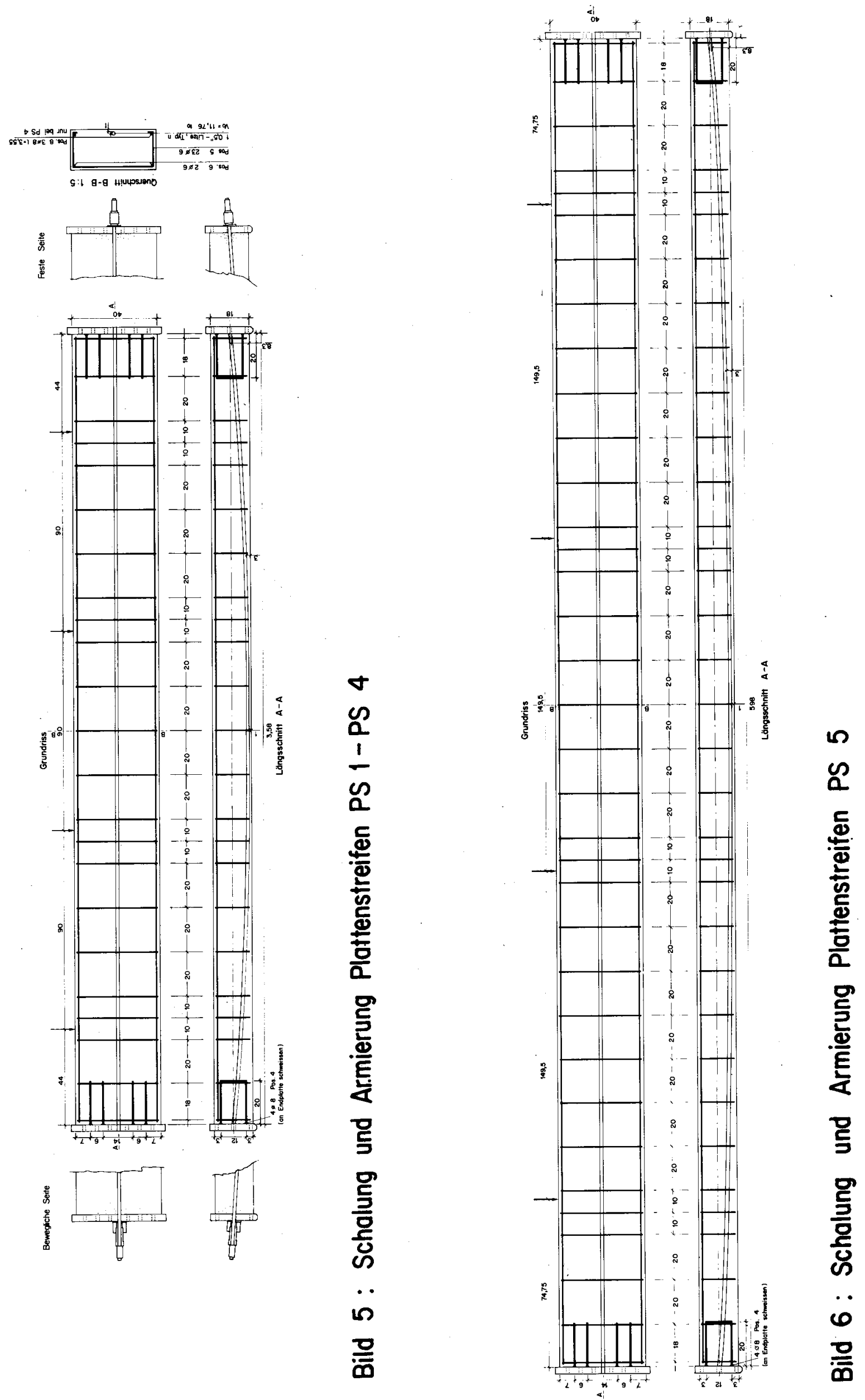


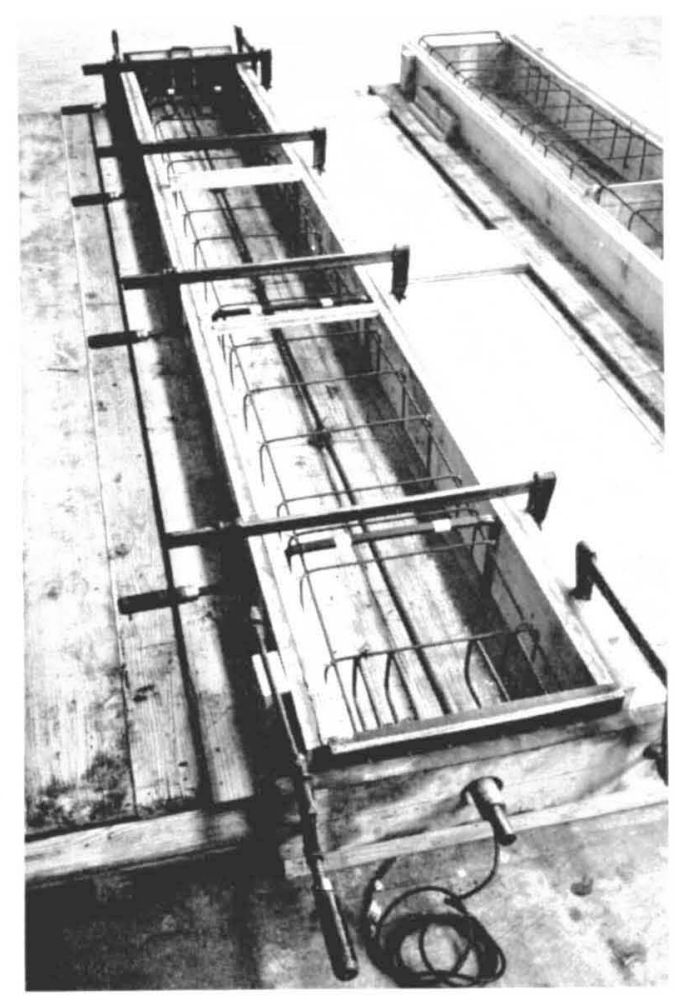

Bild 7: Schalung u. Armierung Plattenstreifen PS 1-3

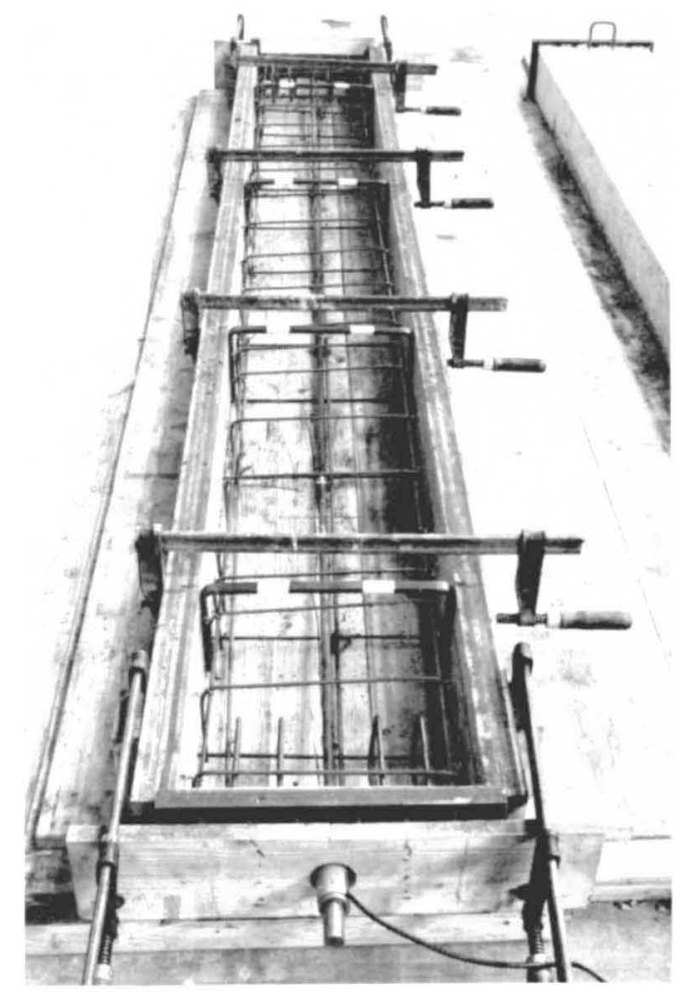

Bild 8: Schalung u. Armierung Plattenstreifen PS 4

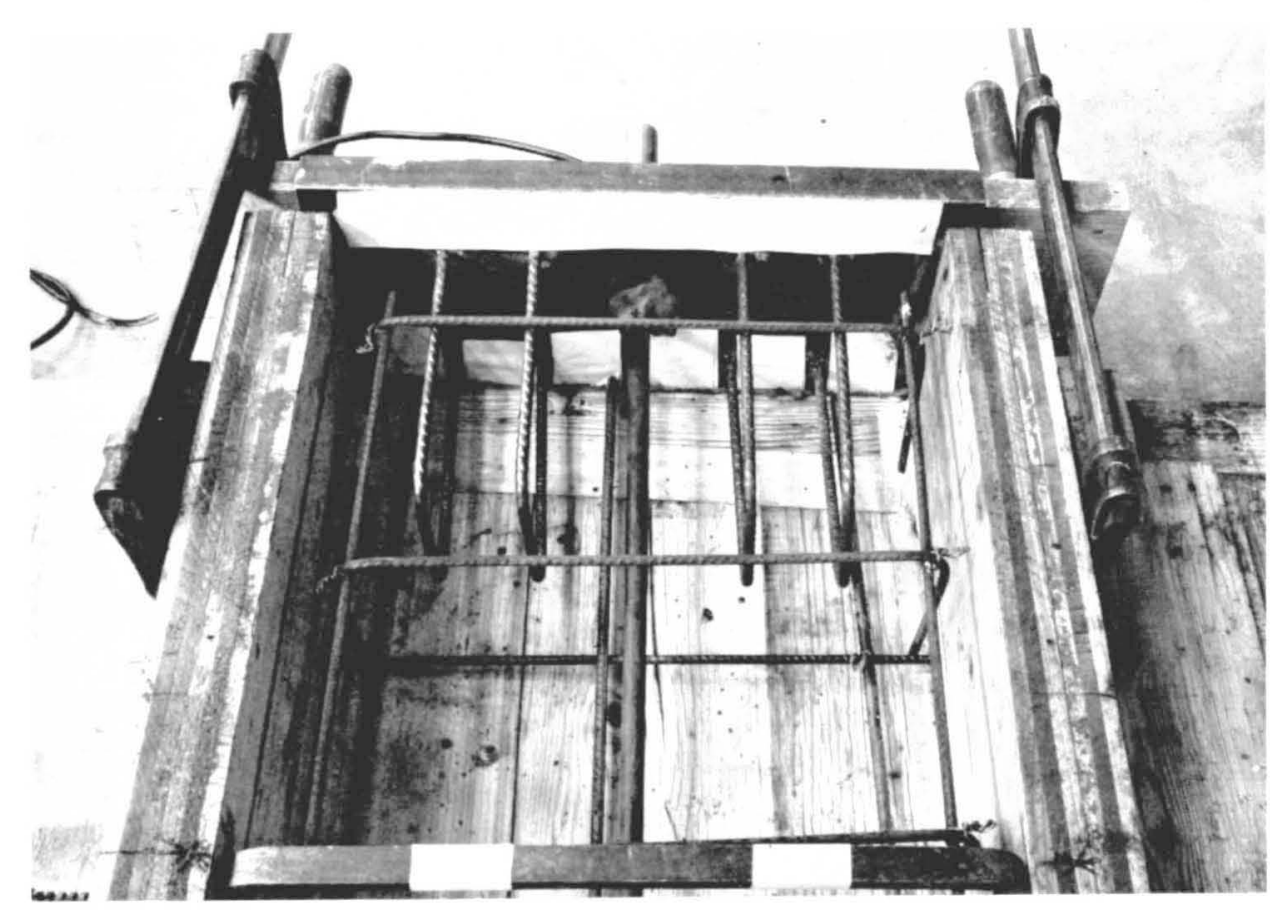

Bild 9: Detail Endplatte 

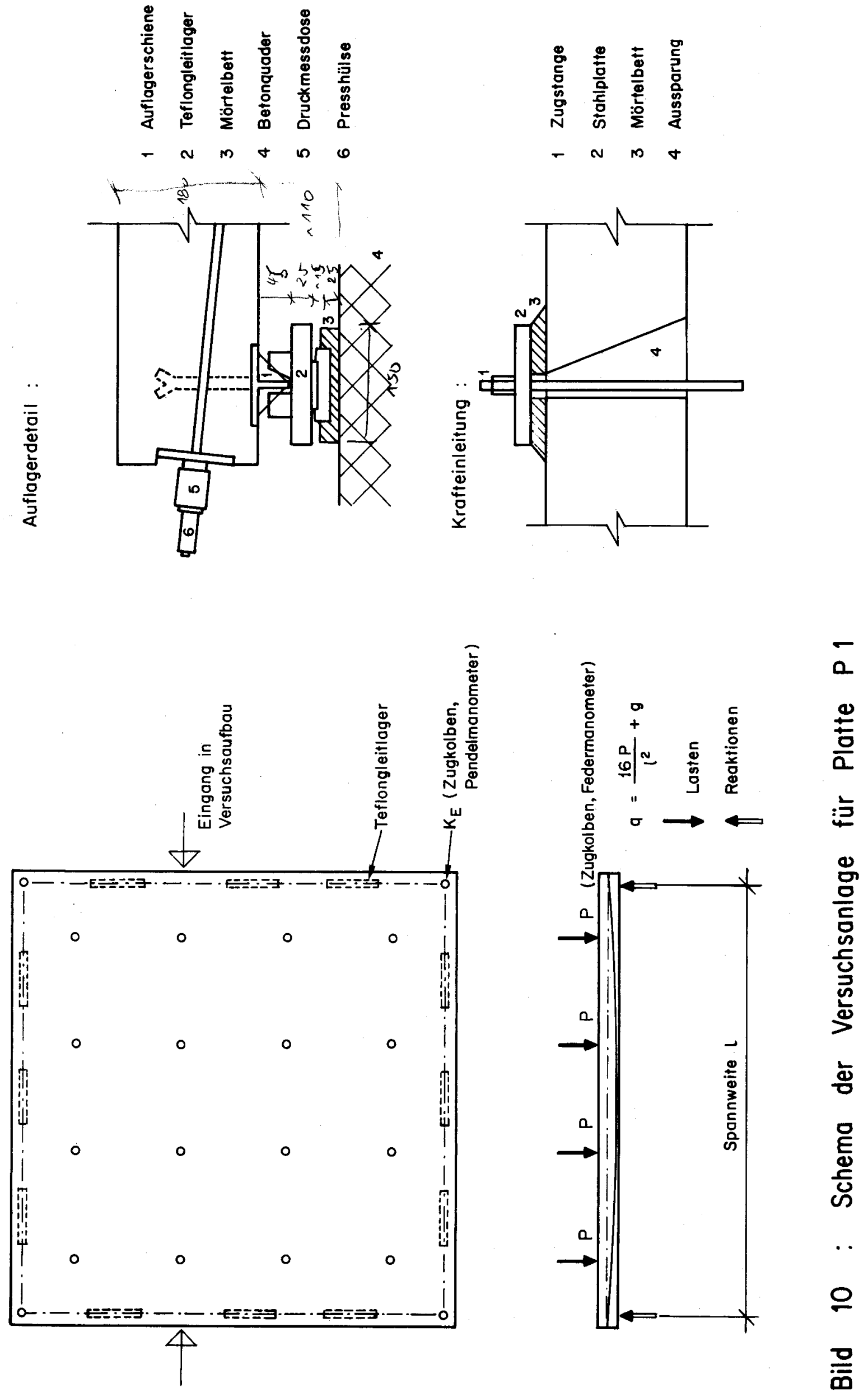

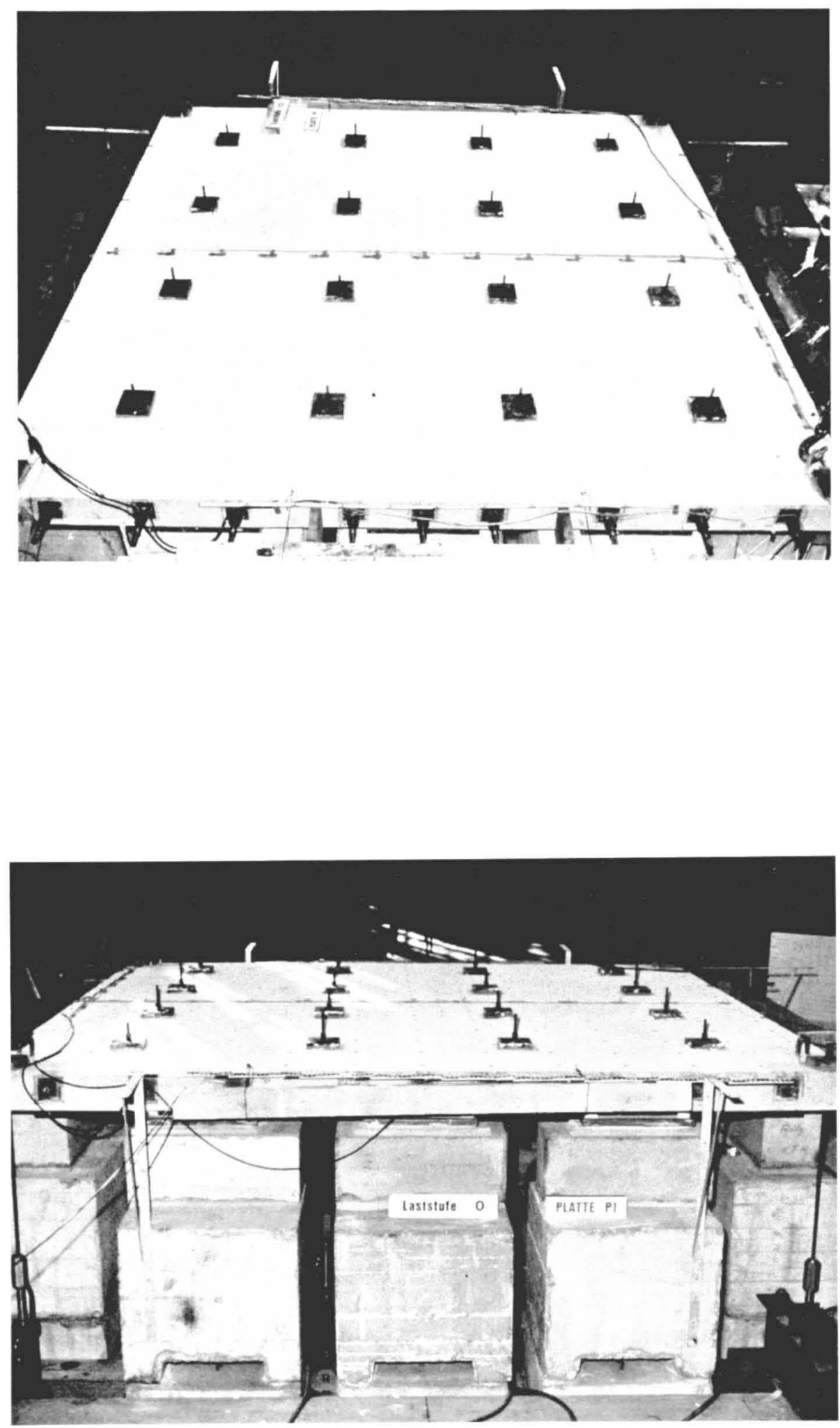

Bild 11: Versuchsanlage Platte P 1 

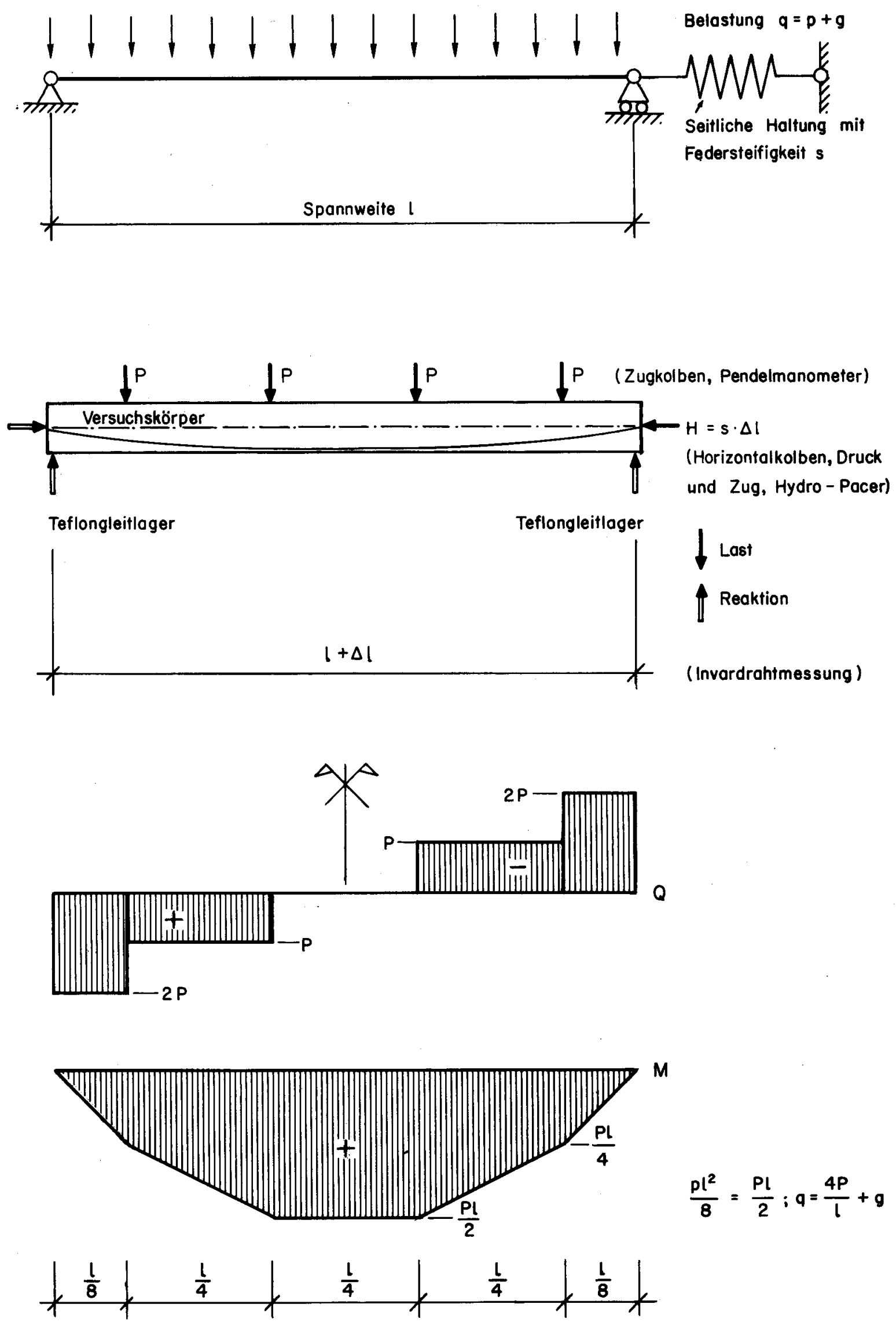

Bild 12 : Schema der Versuchsanlage für Plattenstreifen 

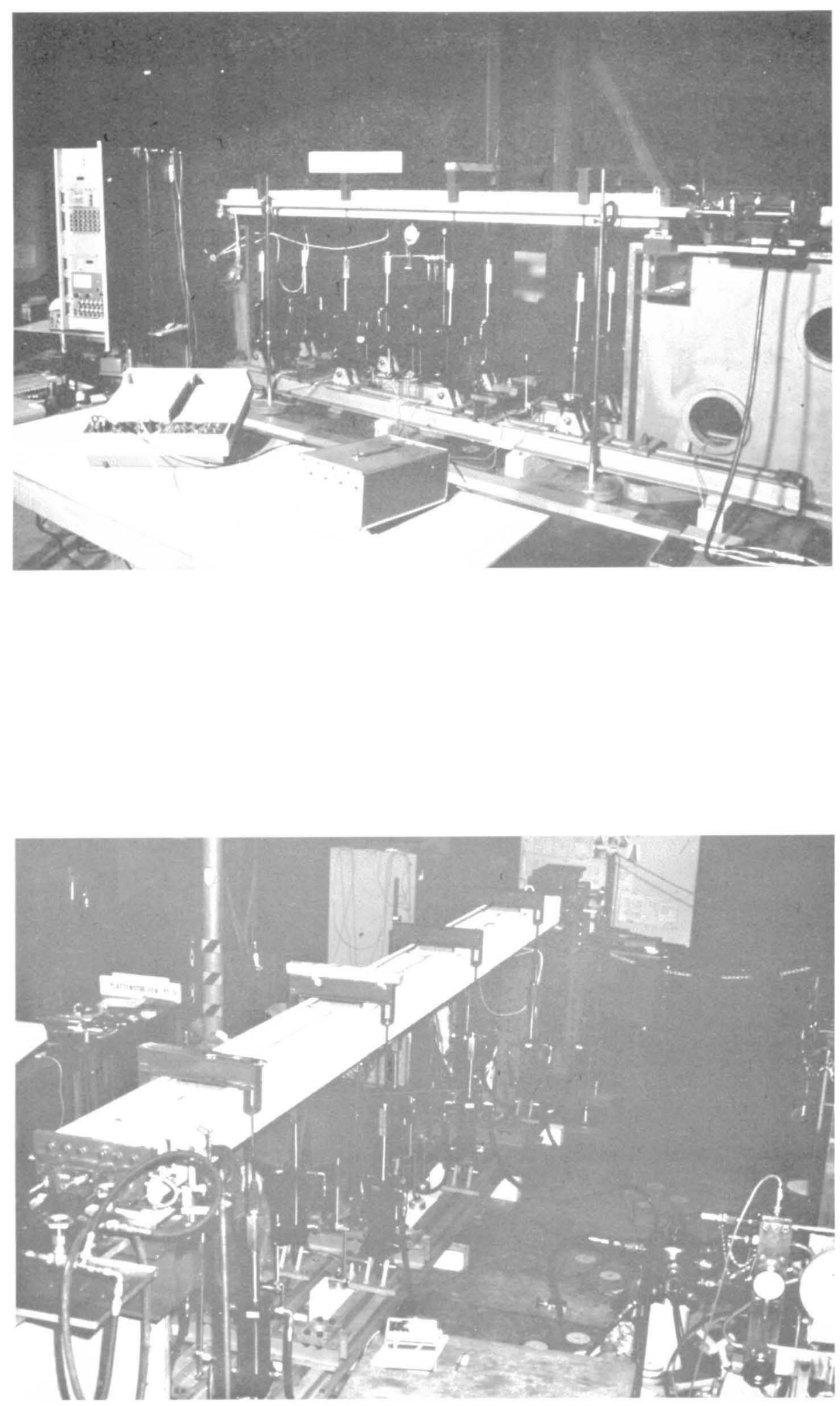

Bild 13: Versuchsanlage Plattenstreifen 

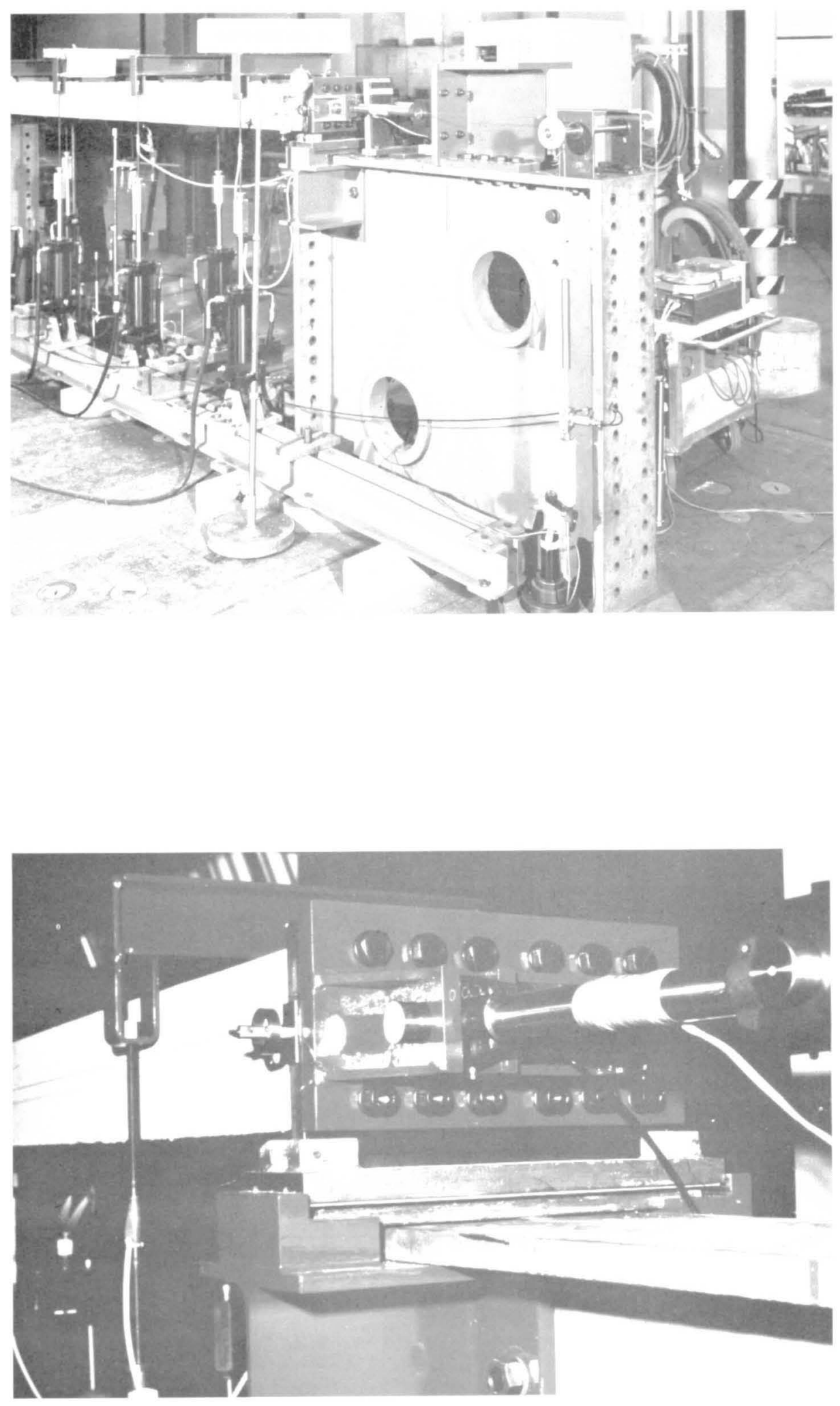

Bild 14: Versuchsanlage Plattenstreifen 


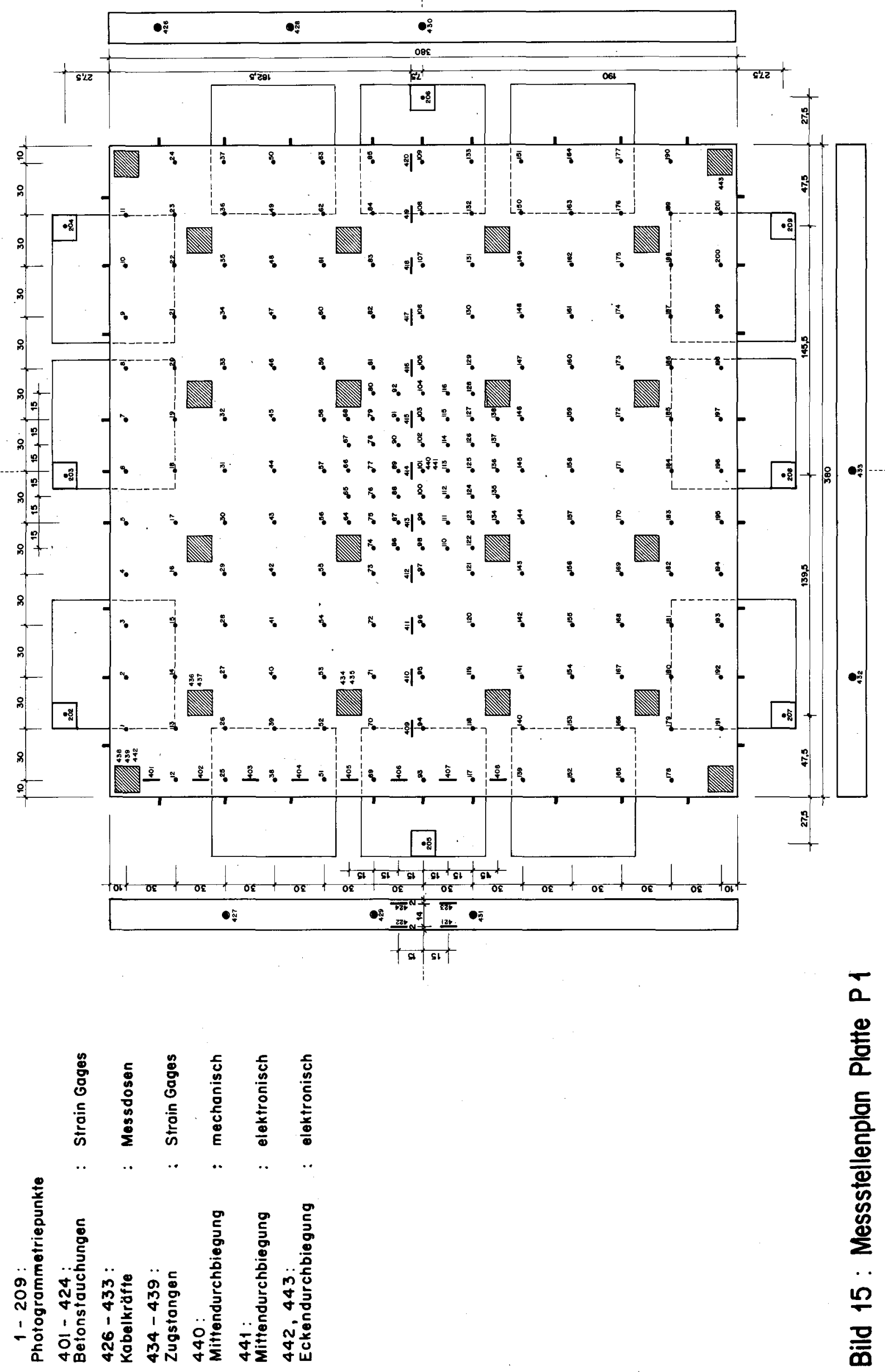




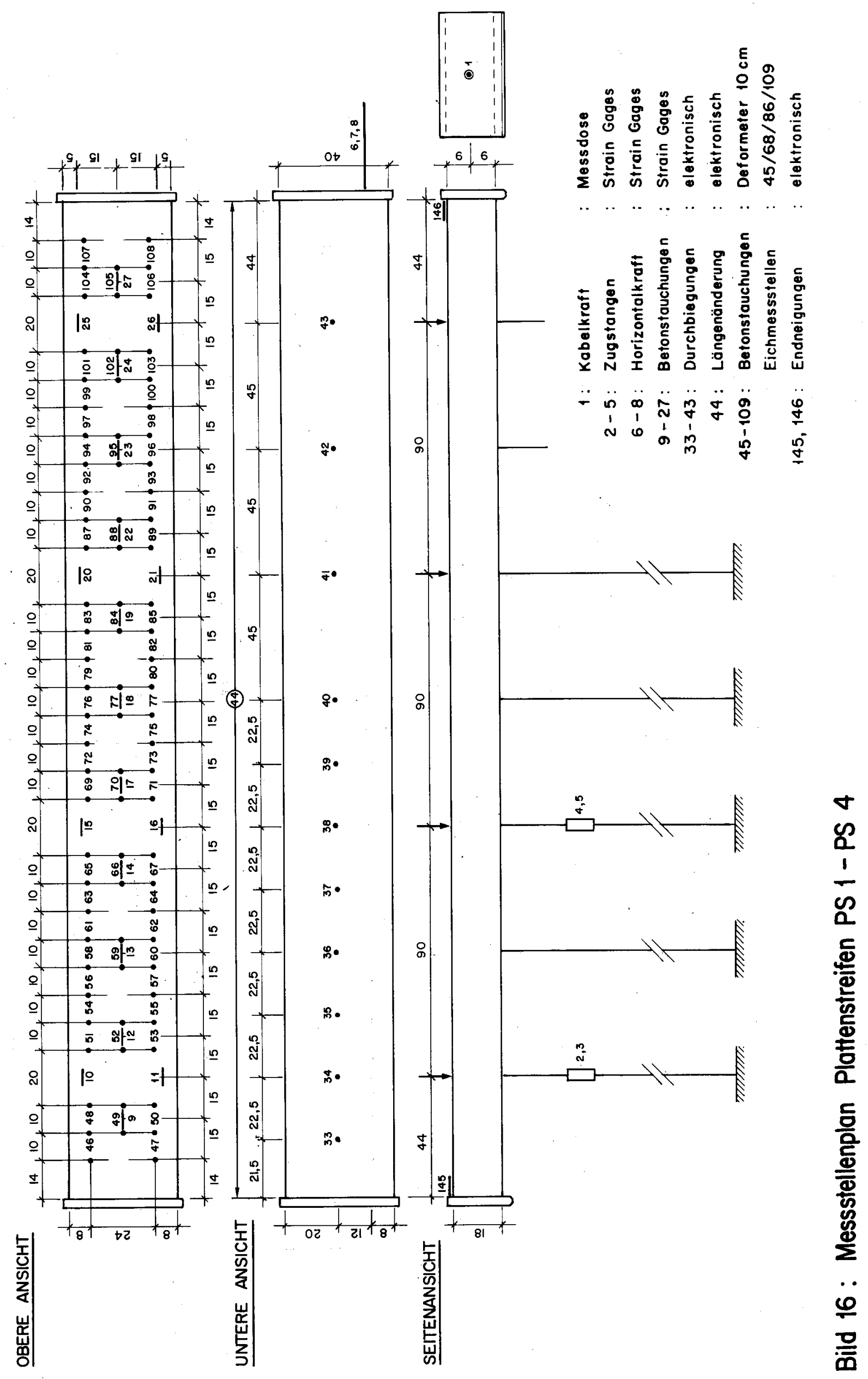




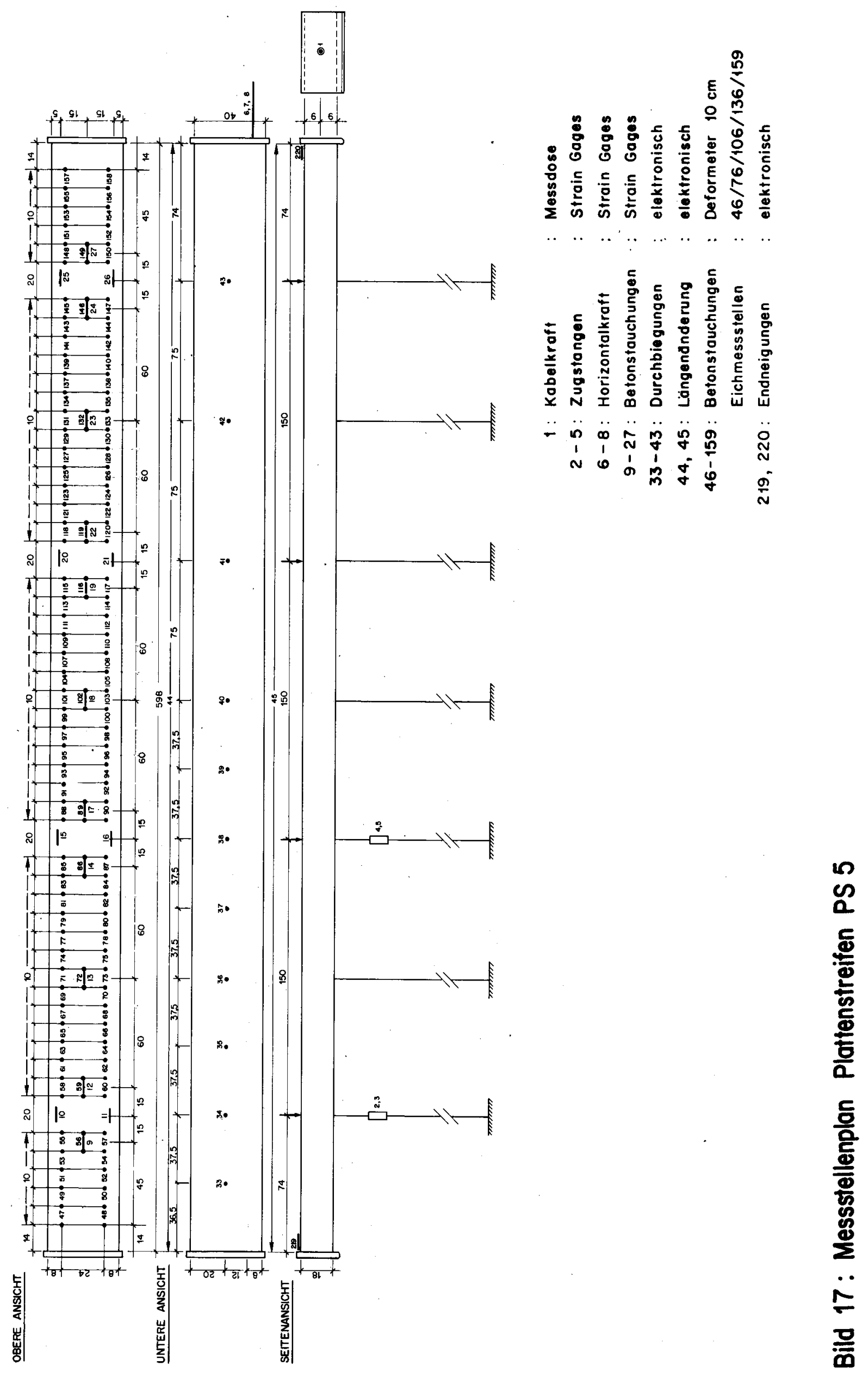




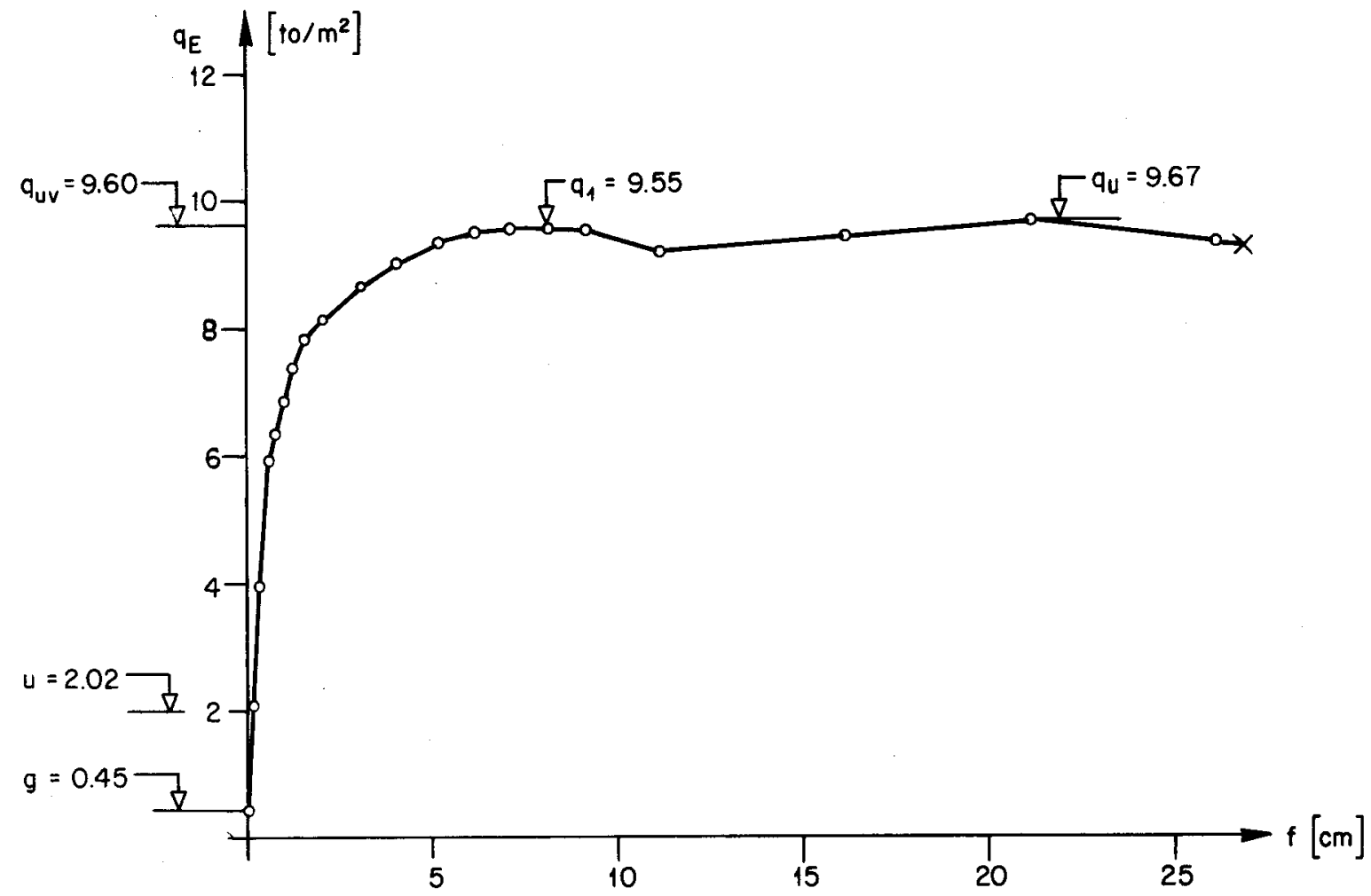

Bild 18: Belastung Platte P1 in Funktion der. Mittendurchbiegung

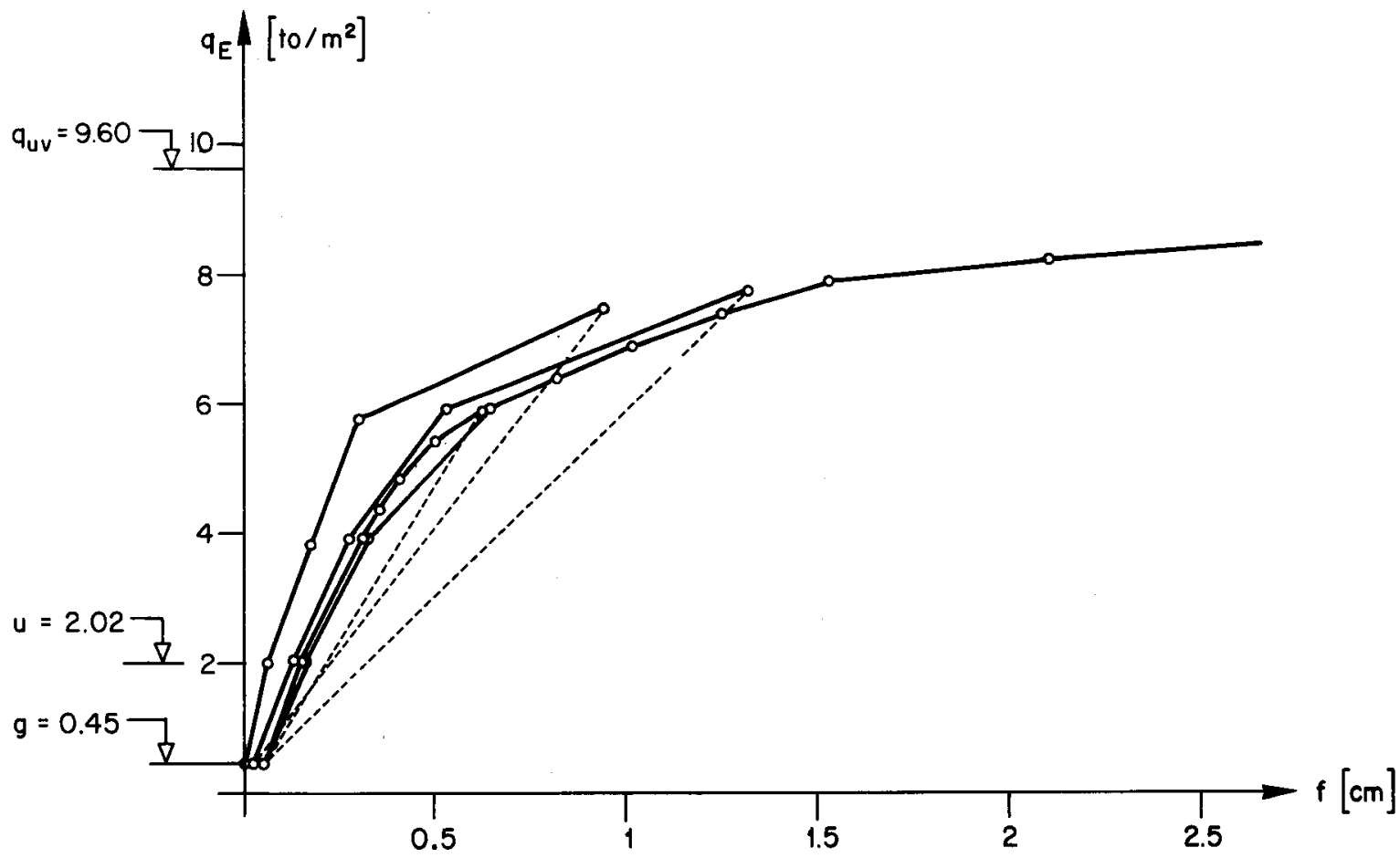

Bild 19: Belastung Platte P1 in Funktion der Mittendurchbiegung (Anfangsbereich) 


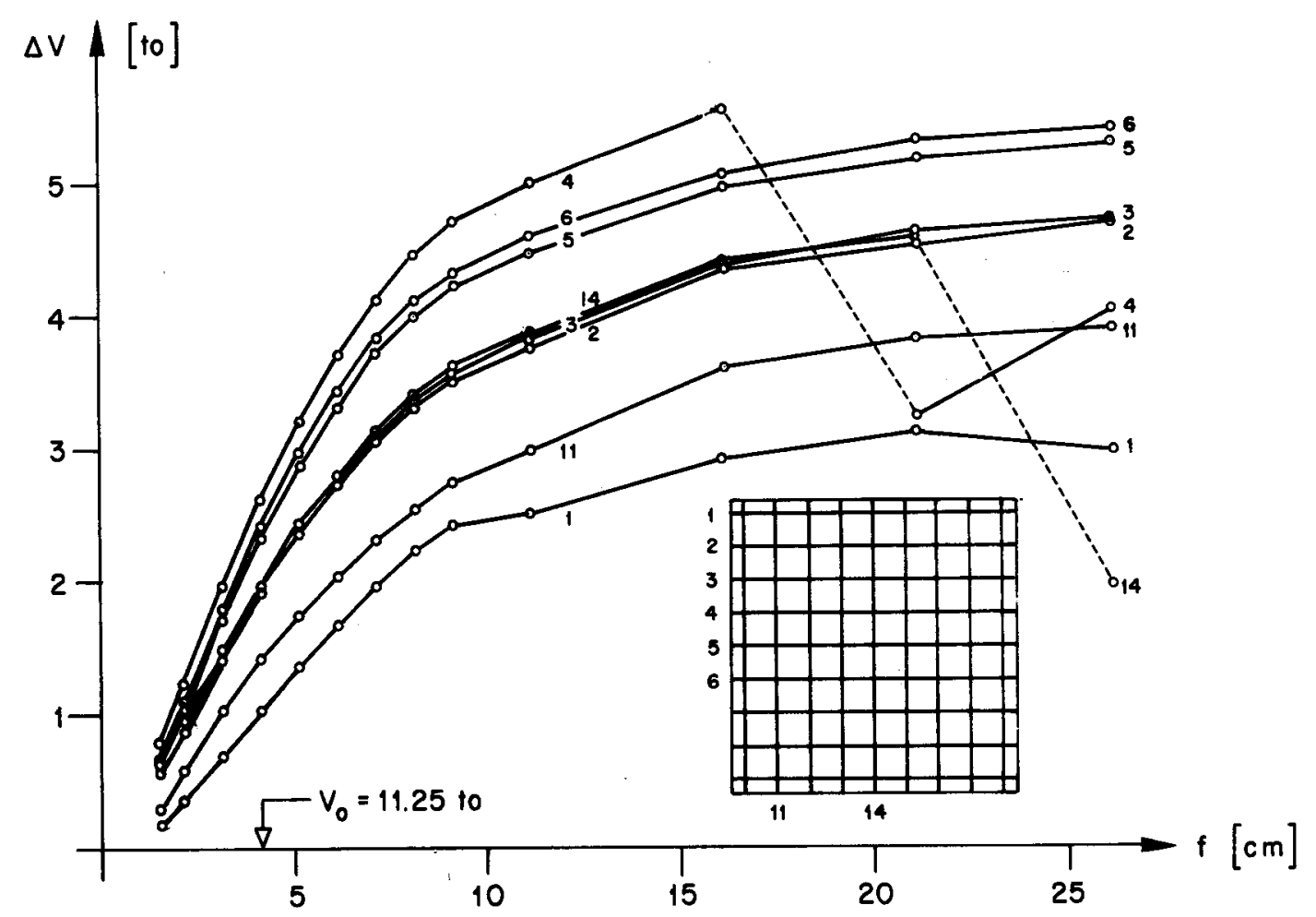

Bild 20: Kabelkraftzuwachs Platte $P 1$ in Funktion der Mittendurchbiegung

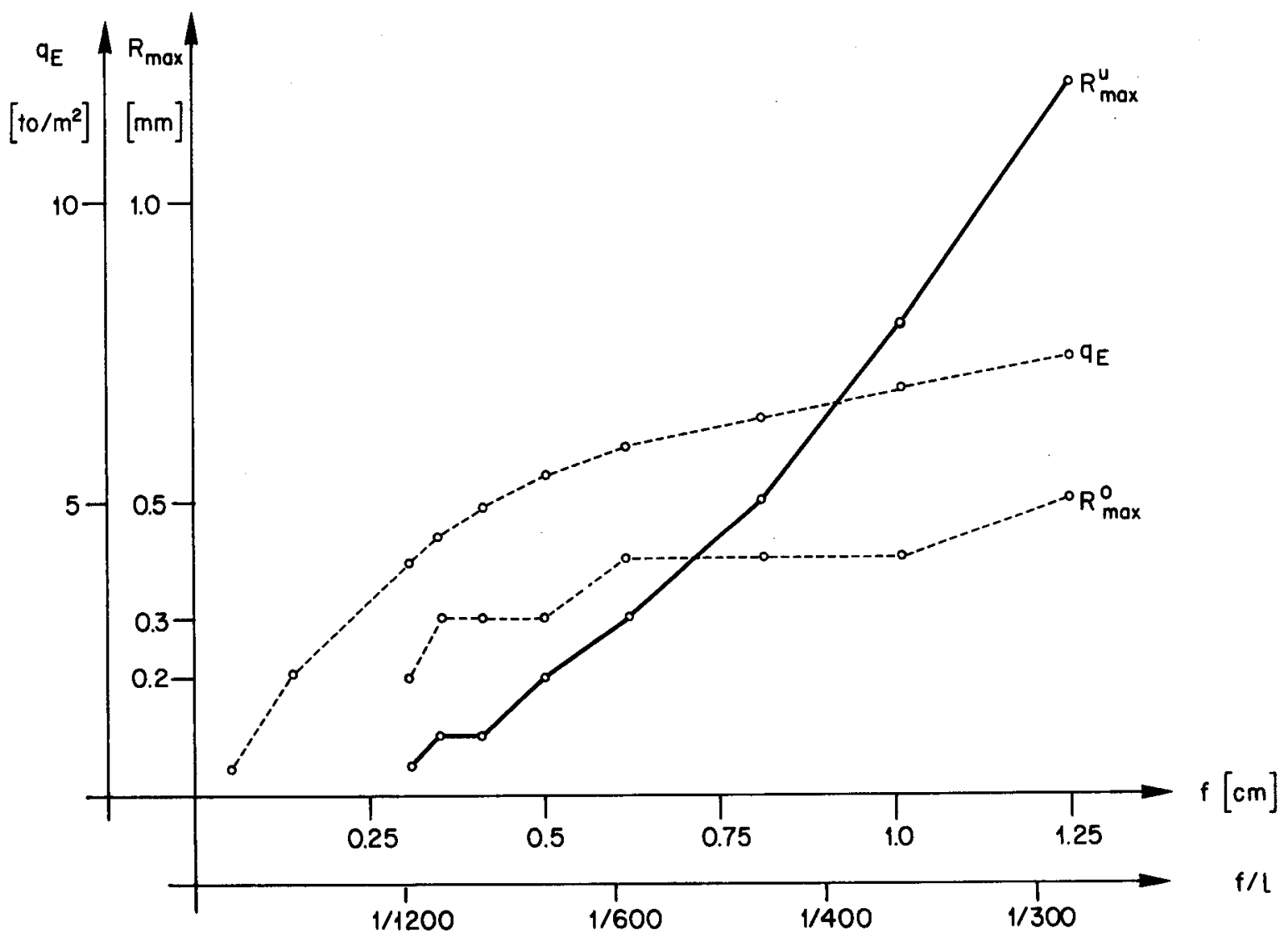

Bild 21: Maximale Rissweiten und Belastung Platte $\mathrm{P} 1$ in Funktion der Mittendurchbiegung 


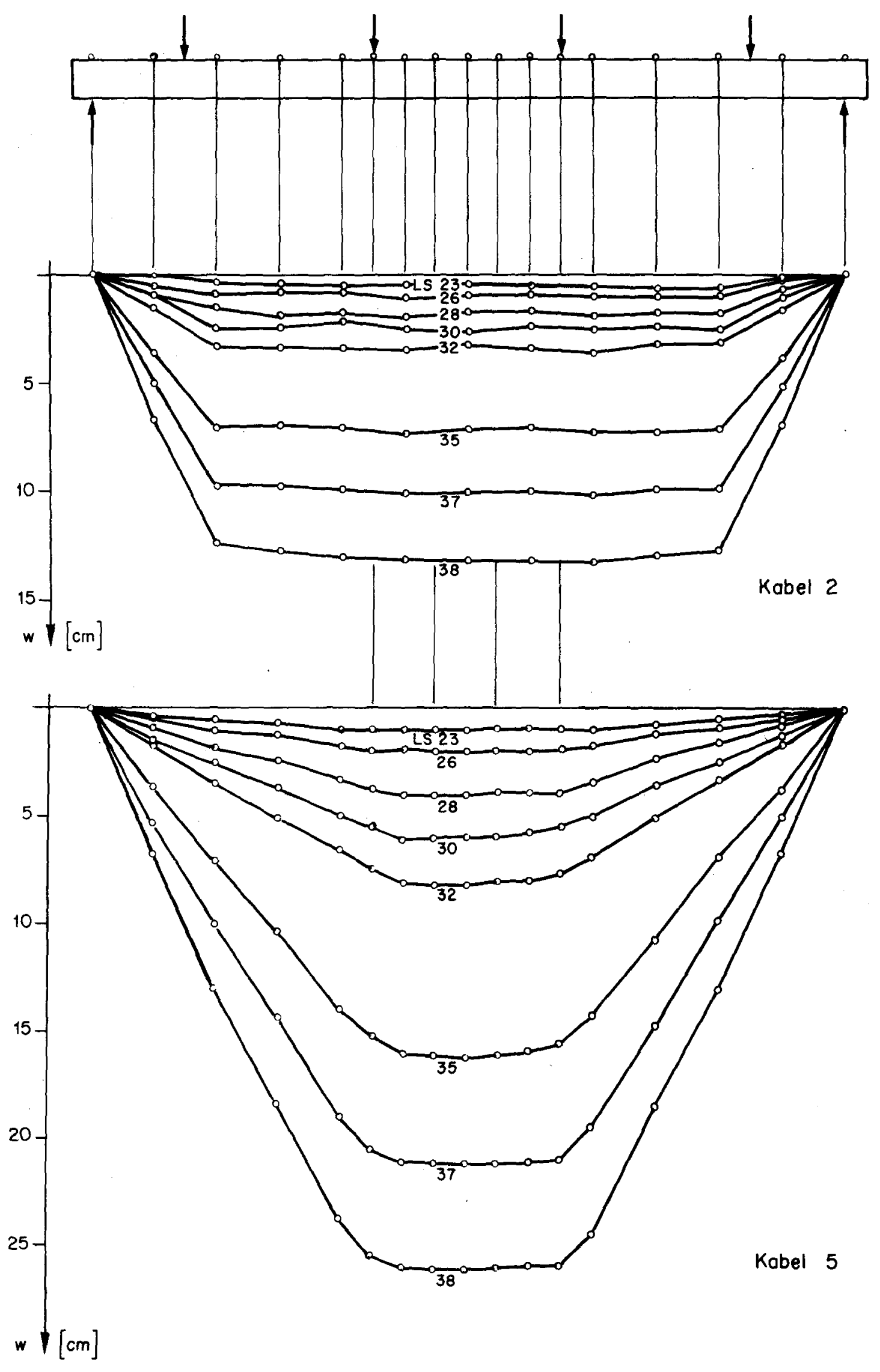

Bild 22: Verlauf der Durchbiegungen Platte $P 1$ entlang Kabel 2 und 5 

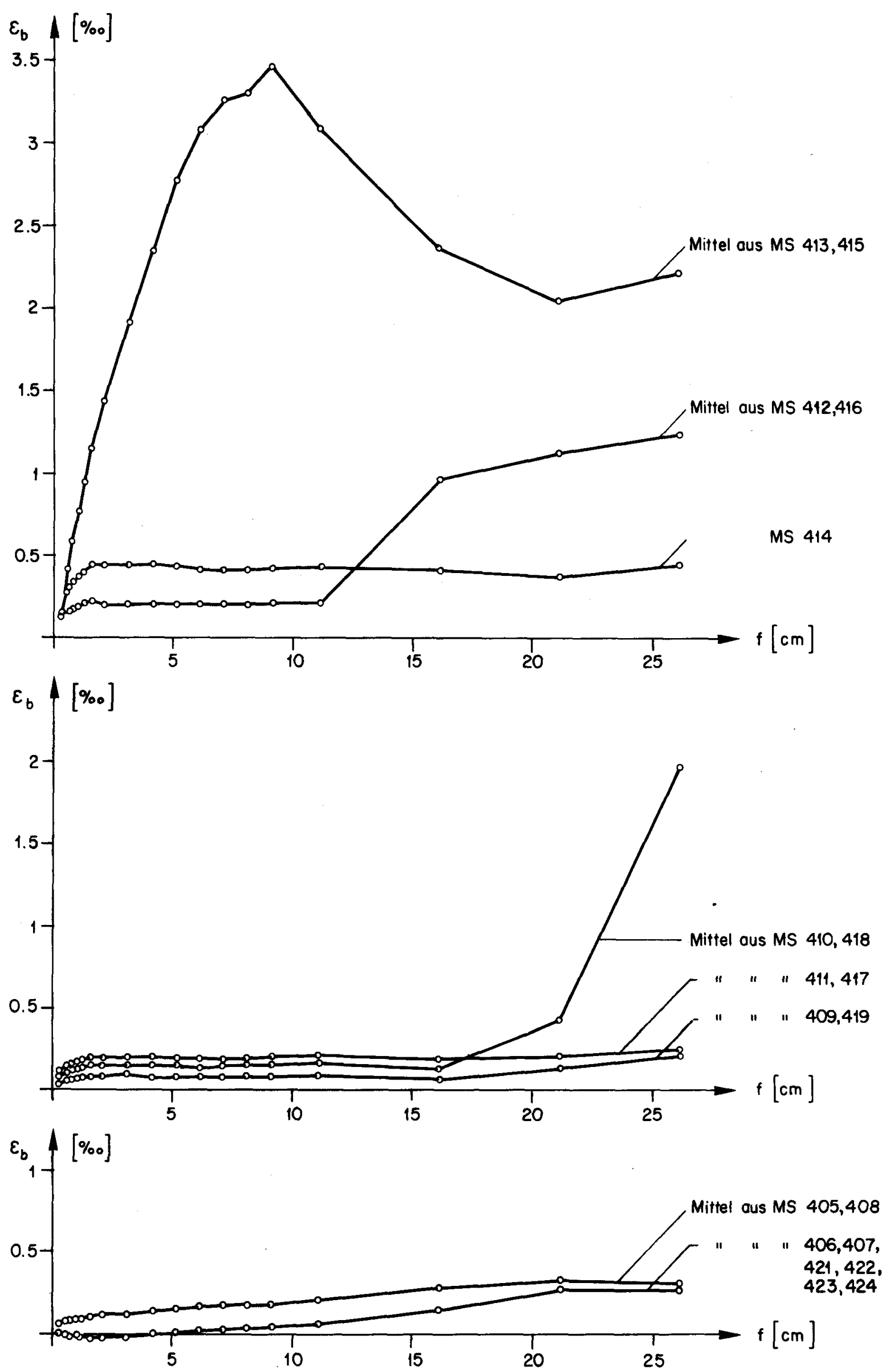

Bild 23: Betonstauchungen Platte $P 1$ in Funktion der Mittendurchbiegung 


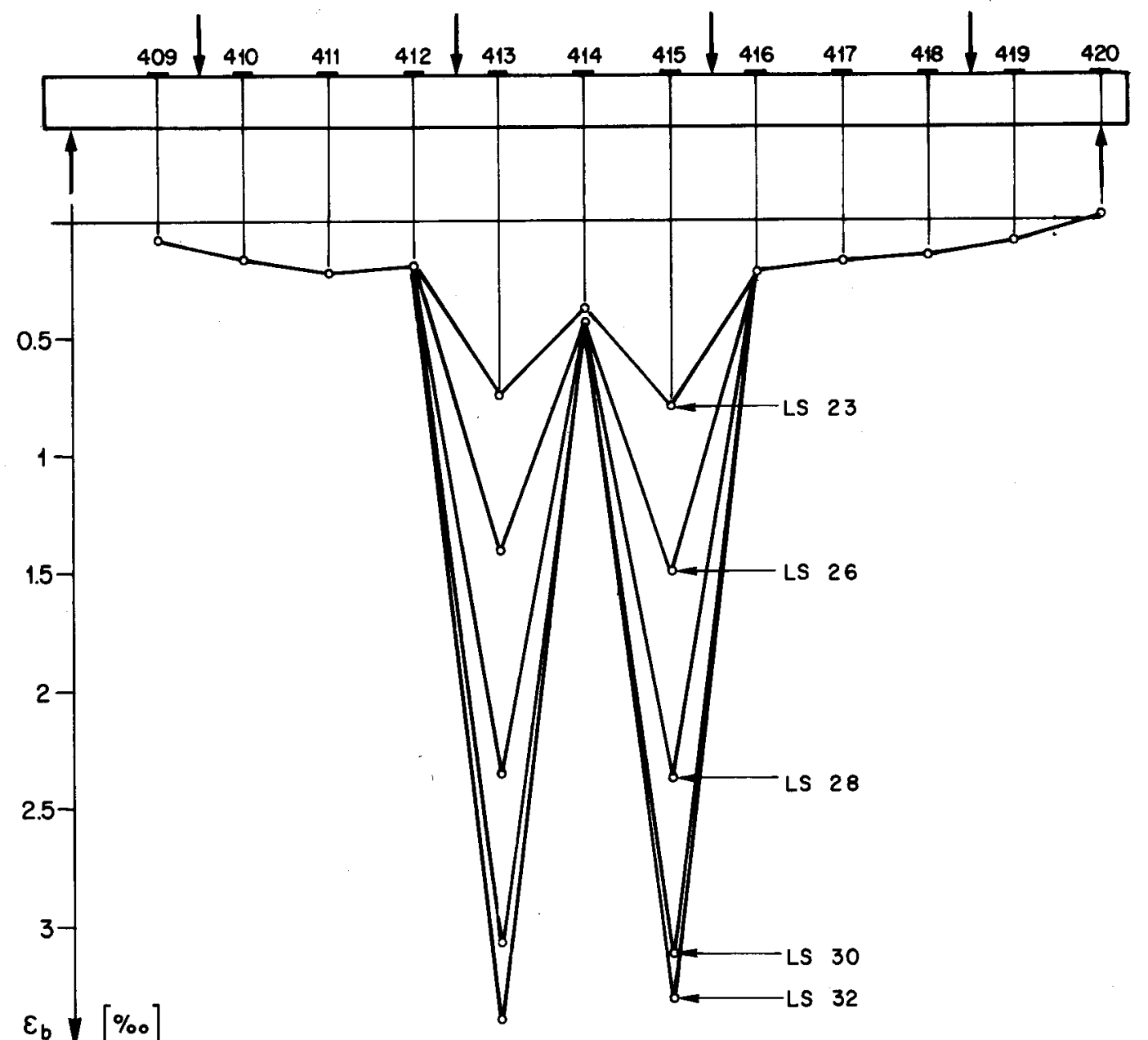

$\varepsilon_{\mathrm{b}} \bigvee[\% \circ]$

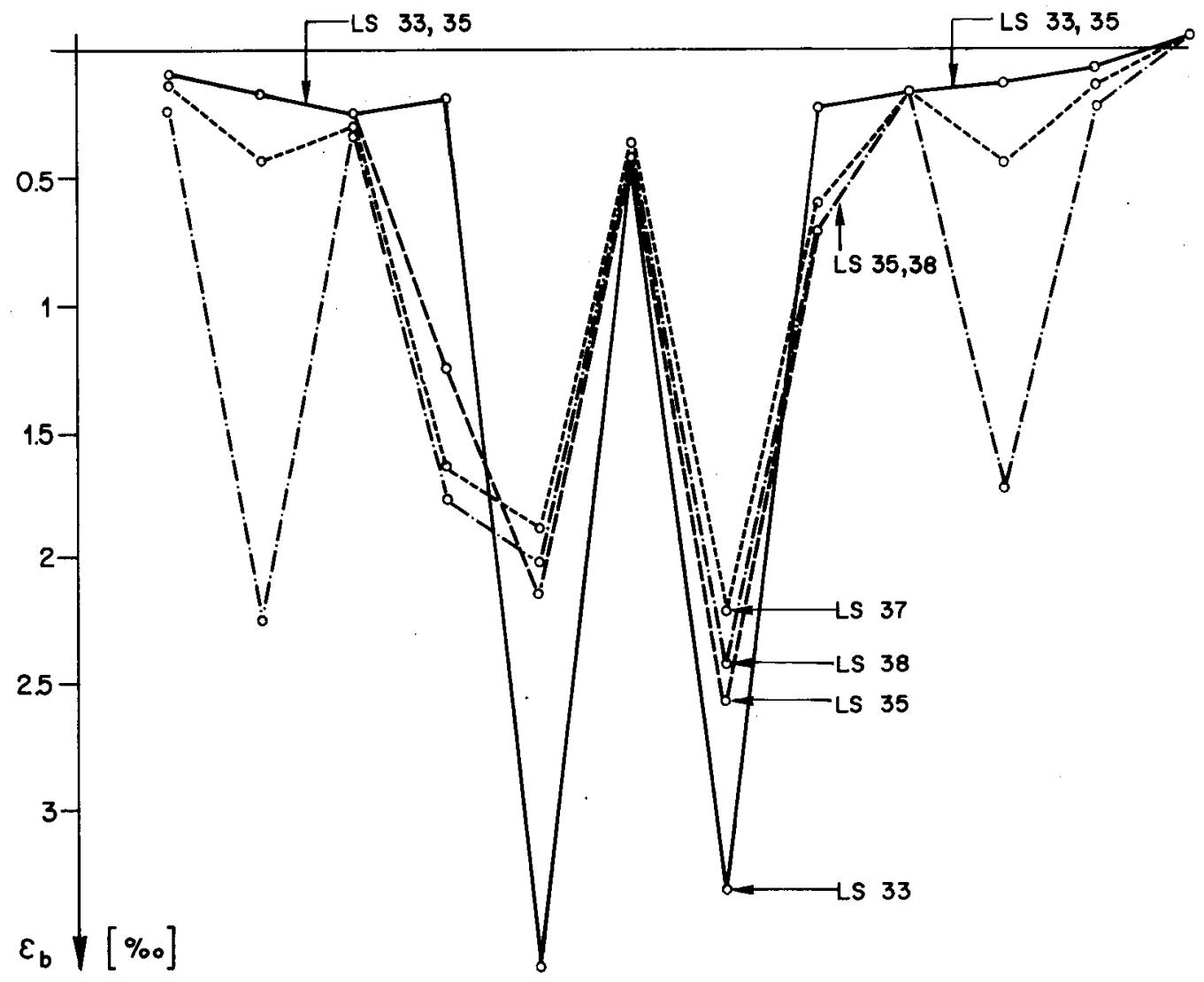

Bild 24 : Verlauf der Betonstauchungen Platte P 1 


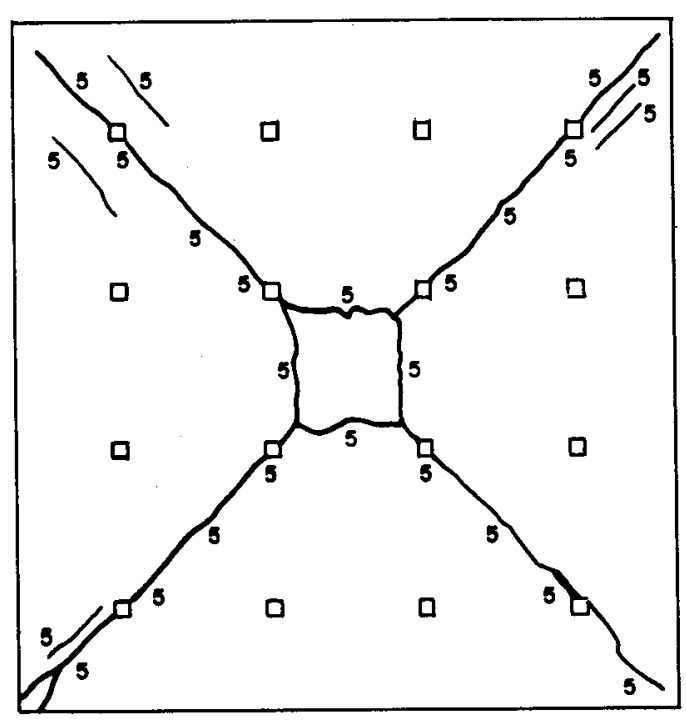

LS 12 Unterseite

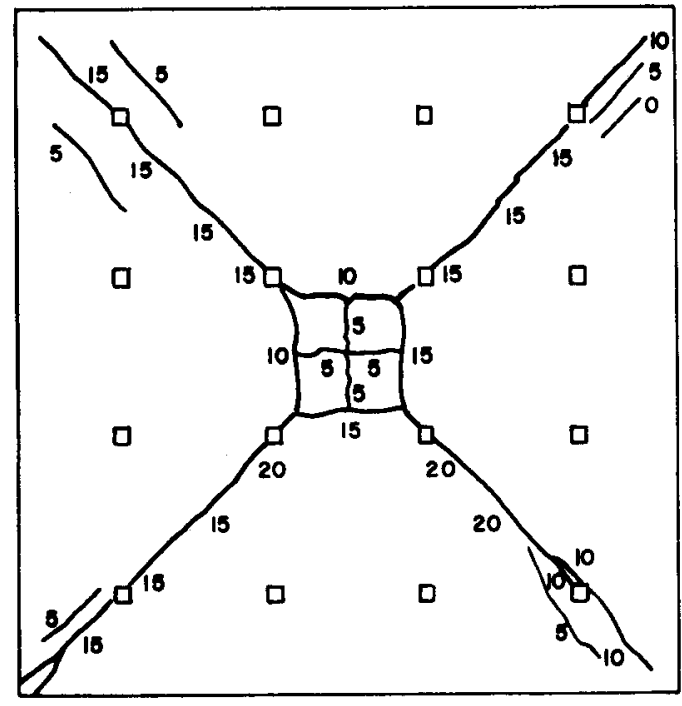

LS 15 Unterseite

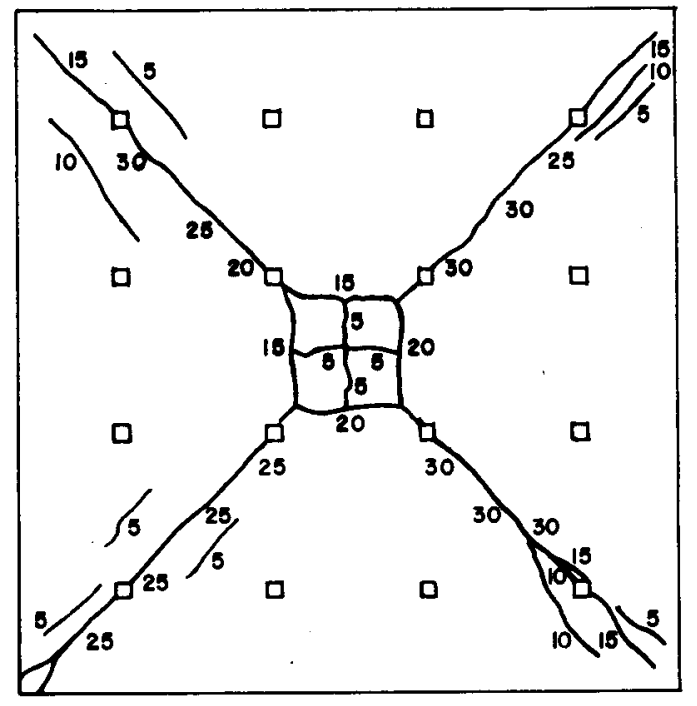

LS 16 Unterseite

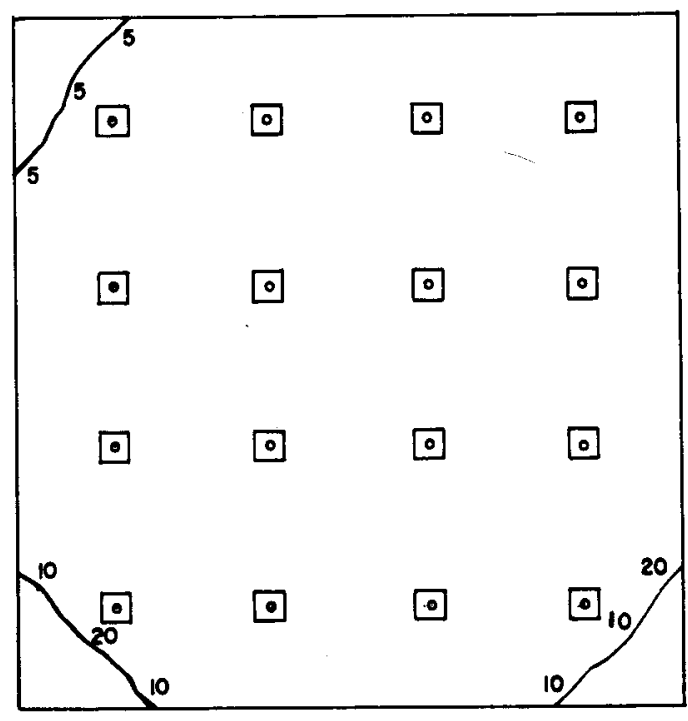

LS 12 Oberseite

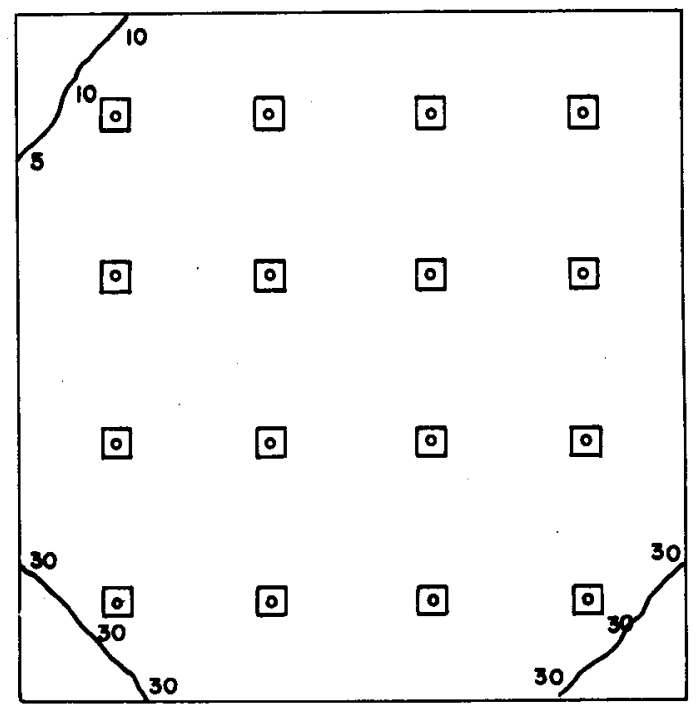

LS 15 Oberseite

$\begin{array}{|cccc|}\int_{3}^{10} 0 & 0 & 0 & 0 \\ 0 & 0 & 0 & 0 \\ 0 & 0 & 0 & 0 \\ \underbrace{0}_{30} & 0 & 0 & 0\end{array}$

LS 16 Oberseite

Bild 25: Rissbilder Platte P 1 (Rissweiten in $1 / 100 \mathrm{~mm}$ ) 


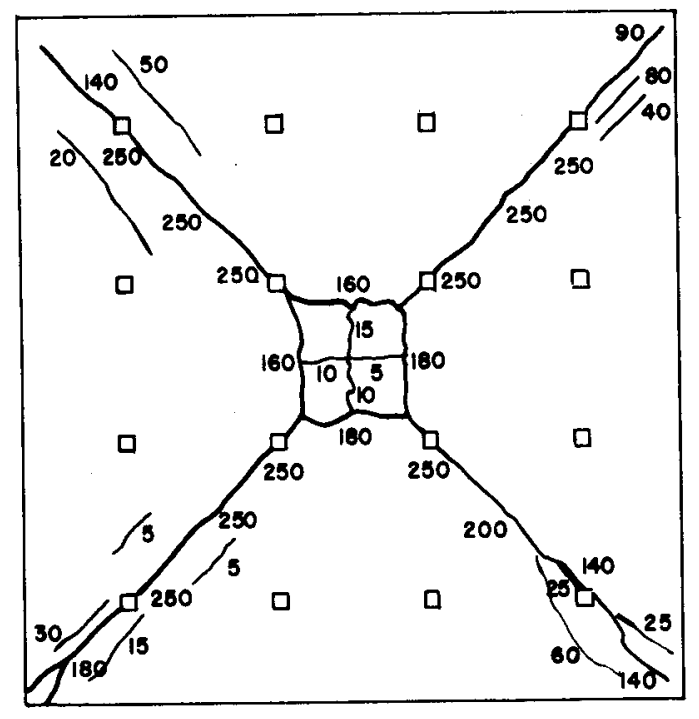

LS 26 Unterseite

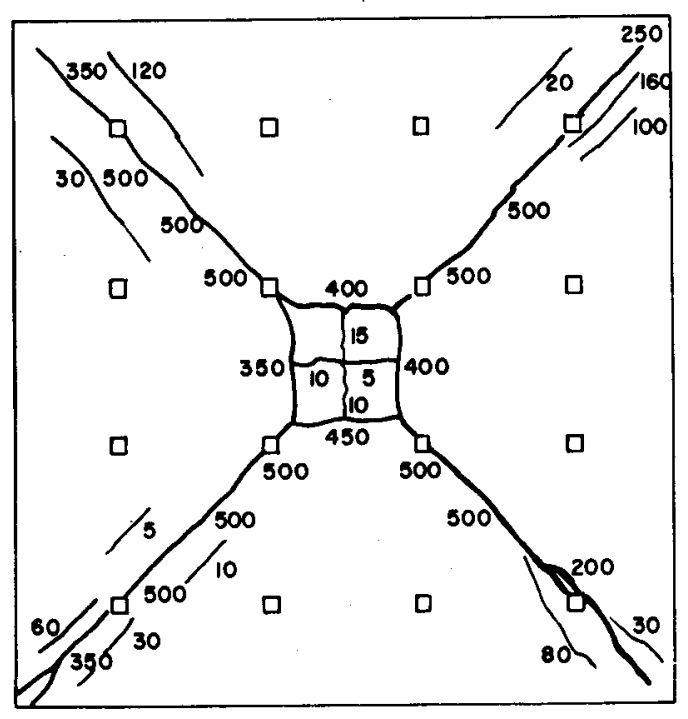

LS 28 Unterseite

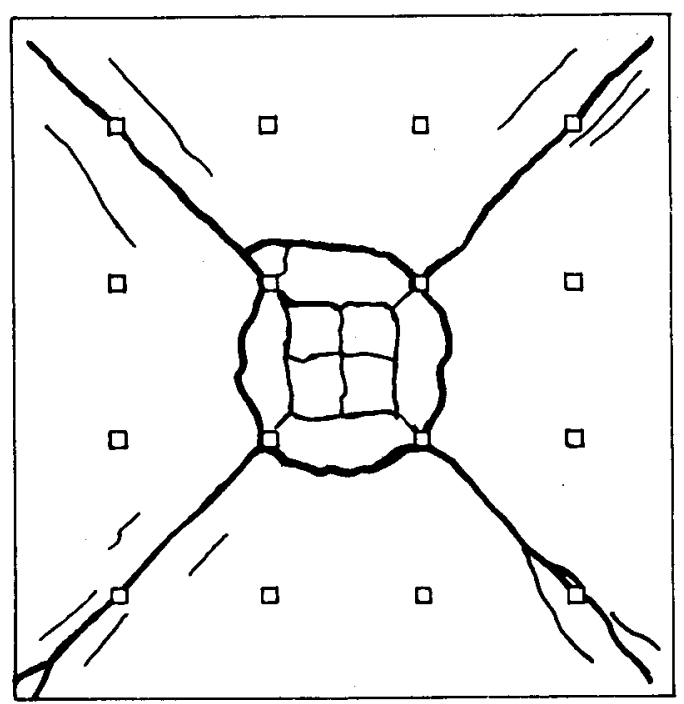

LS 35 Unterseite

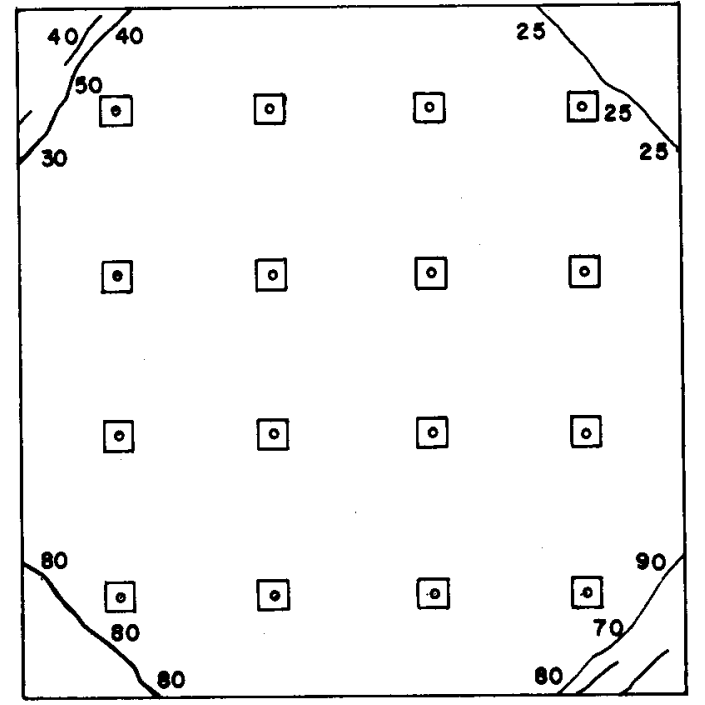

LS 26 Oberseite

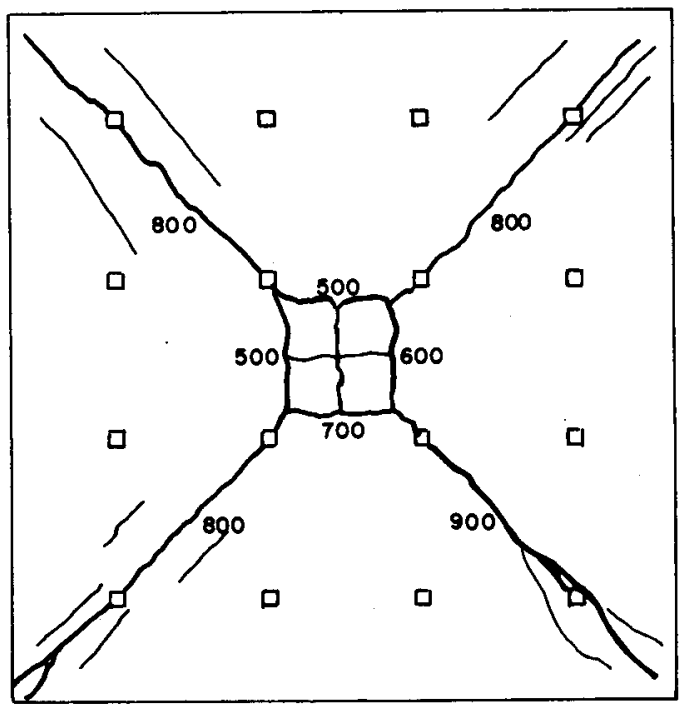

LS 30 Unterseite

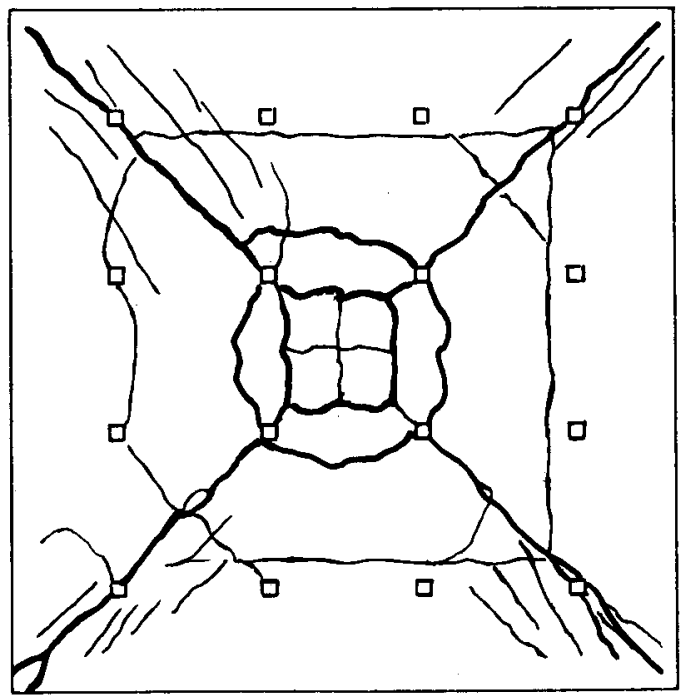

Bruchbild Unterseite

Bild 26: Rissbilder Platte P 1 (Rissweiten in $1 / 100 \mathrm{~mm}$ ) 


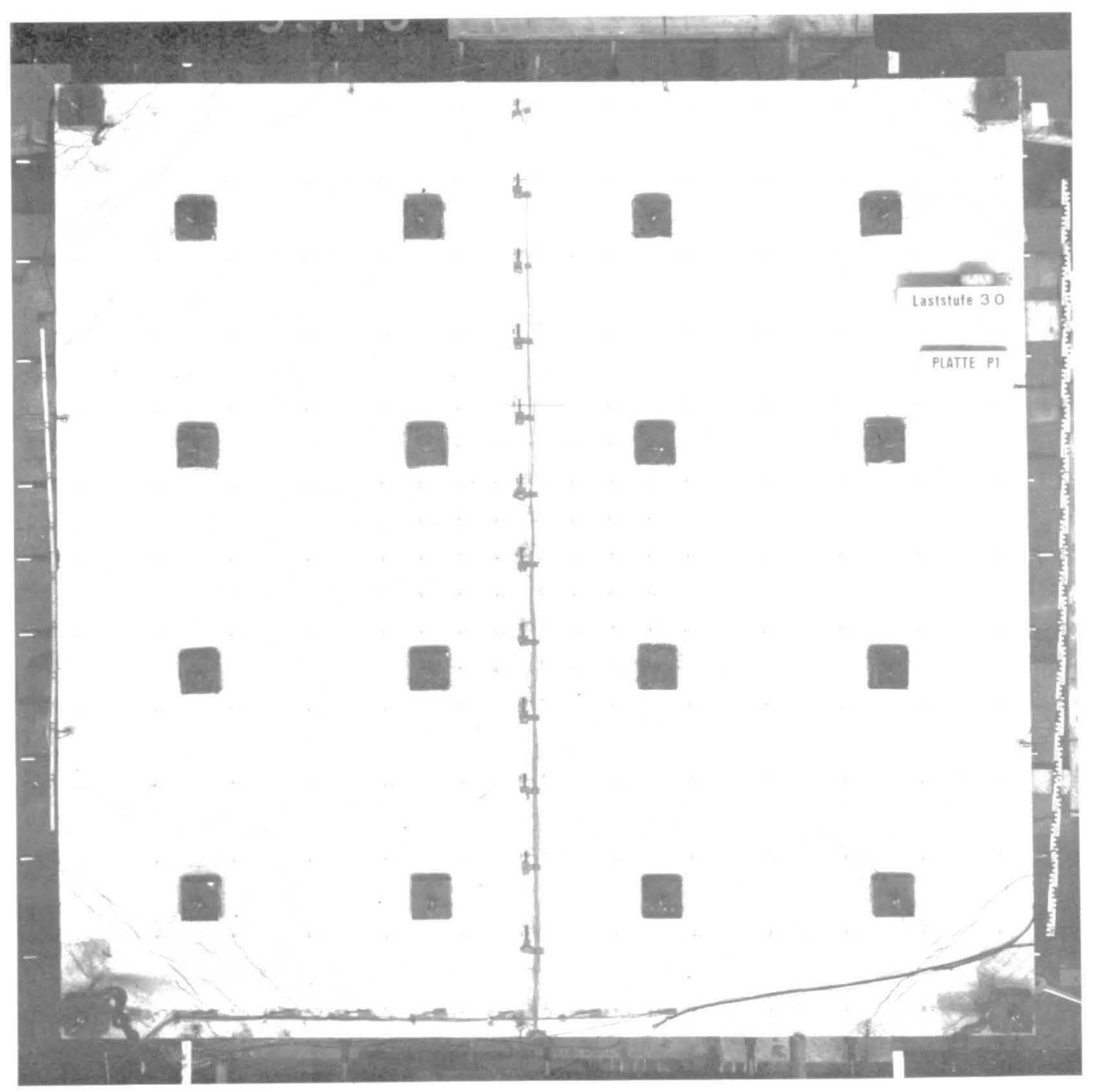

Bild 27 a: Entwicklung Bruchmechanismus Platte P 1 


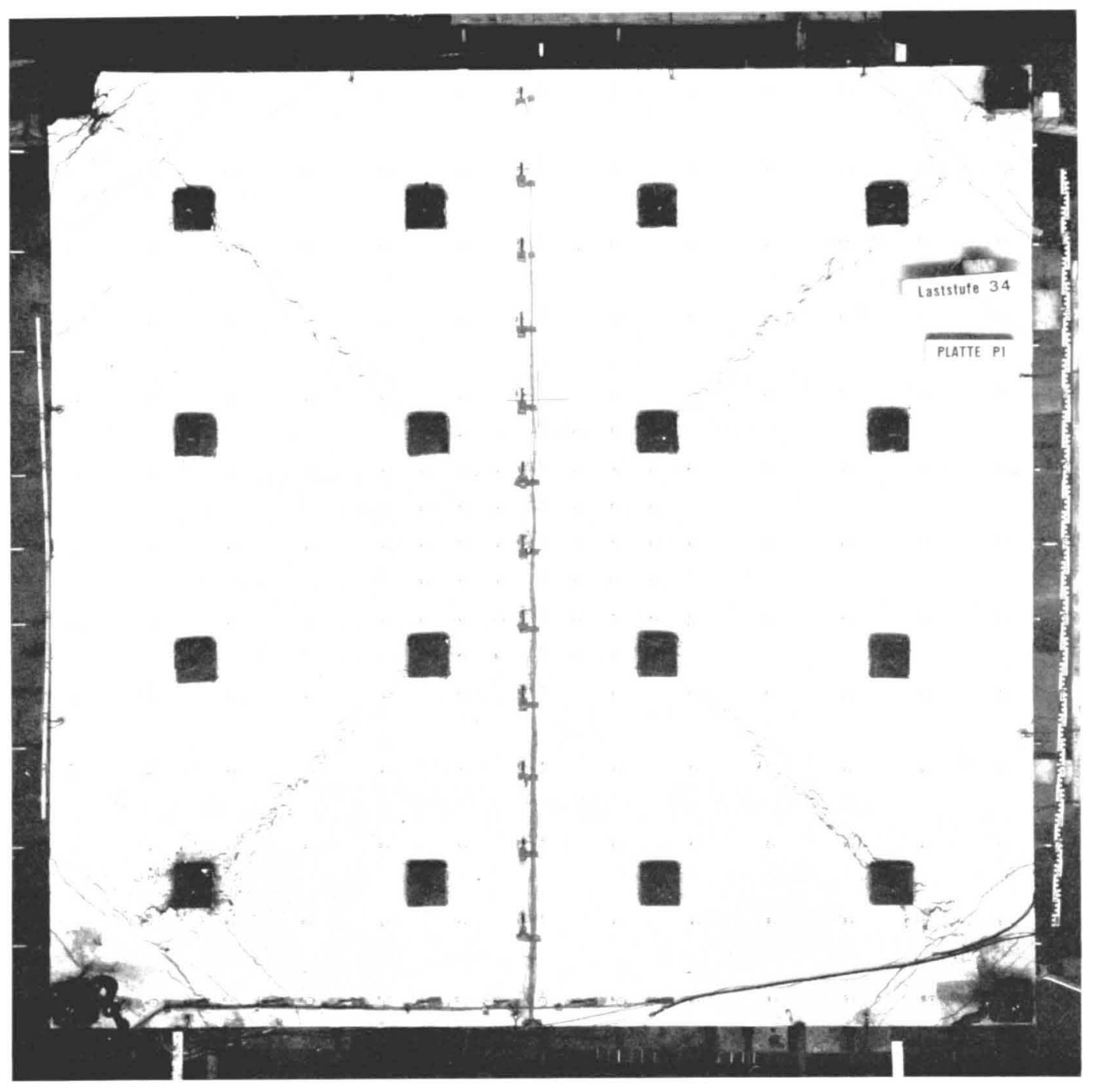

Bild 27 b: Entwicklung Bruchmechanismus Platte P 1 


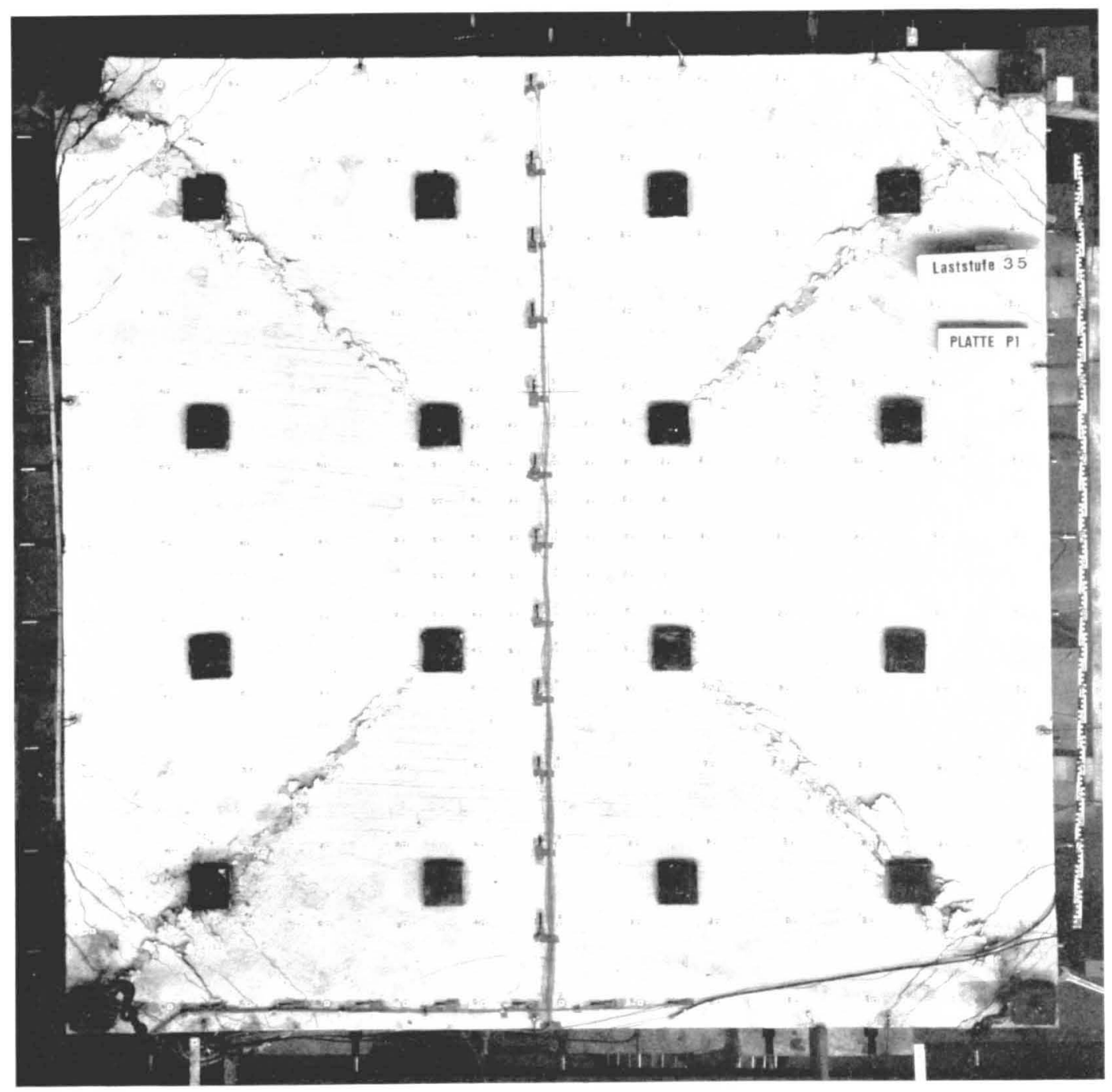

2

Bild $27 \mathrm{c}$ : Entwicklung Bruchmechanismus Platte P 1 


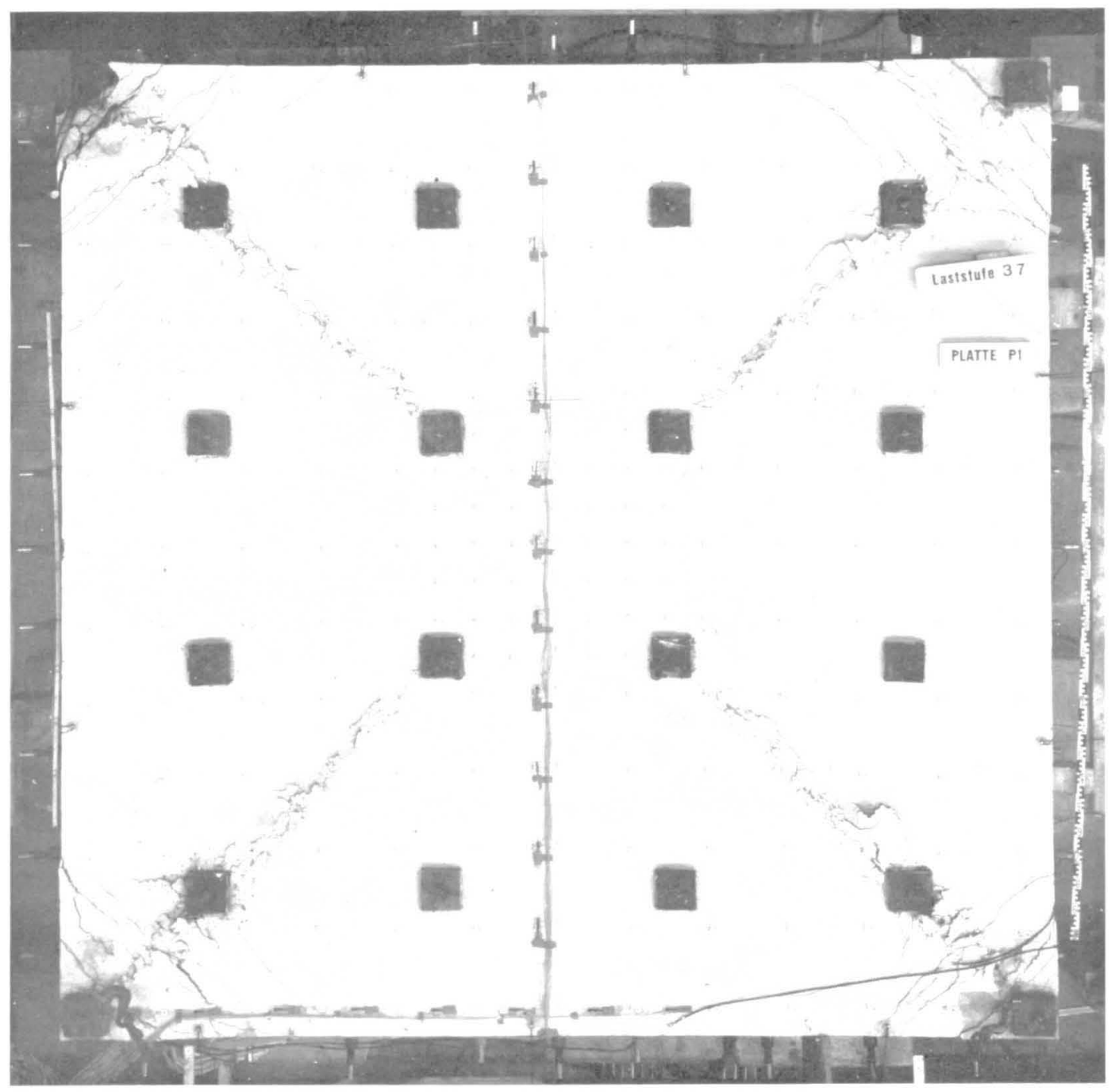

Bild $27 \mathrm{~d}$ : Entwicklung Bruchmechanismus Platte P 1 


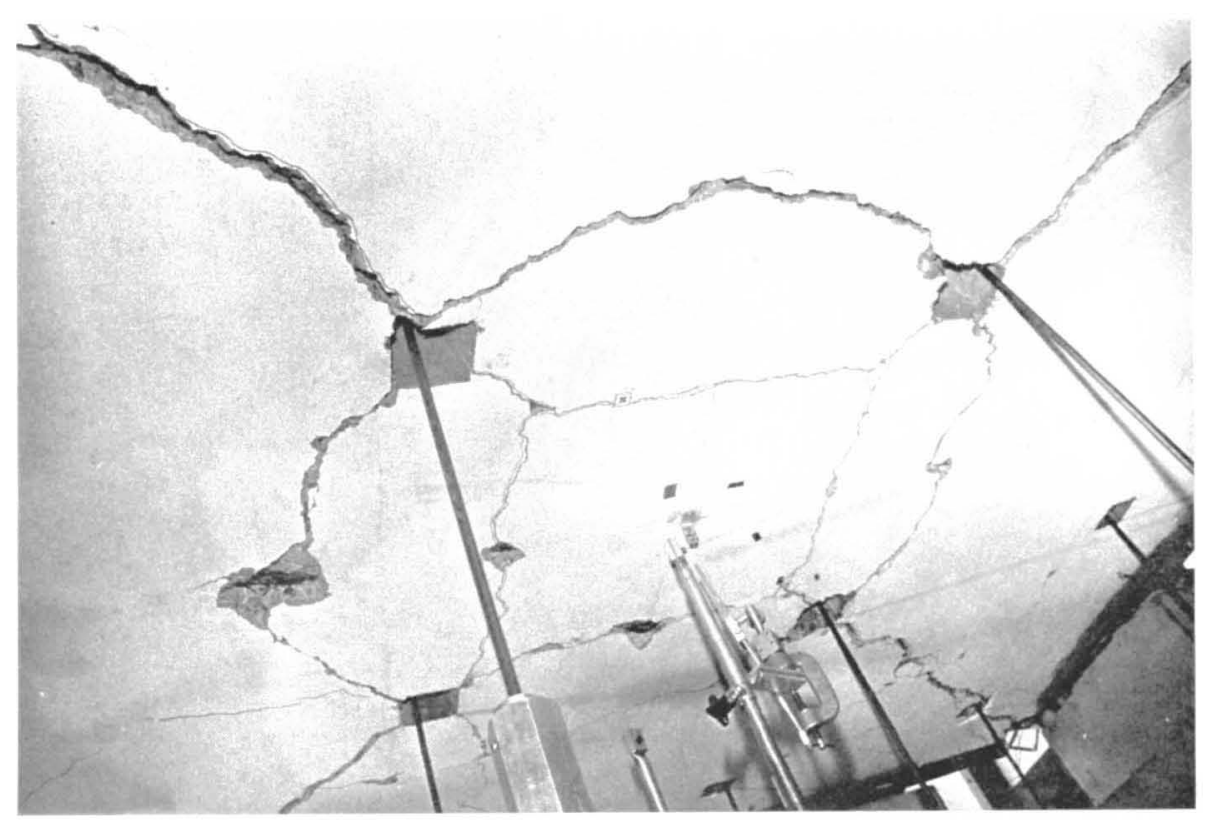

Bild 28: Bruchmechanismus Platte P1 (Unterseite)
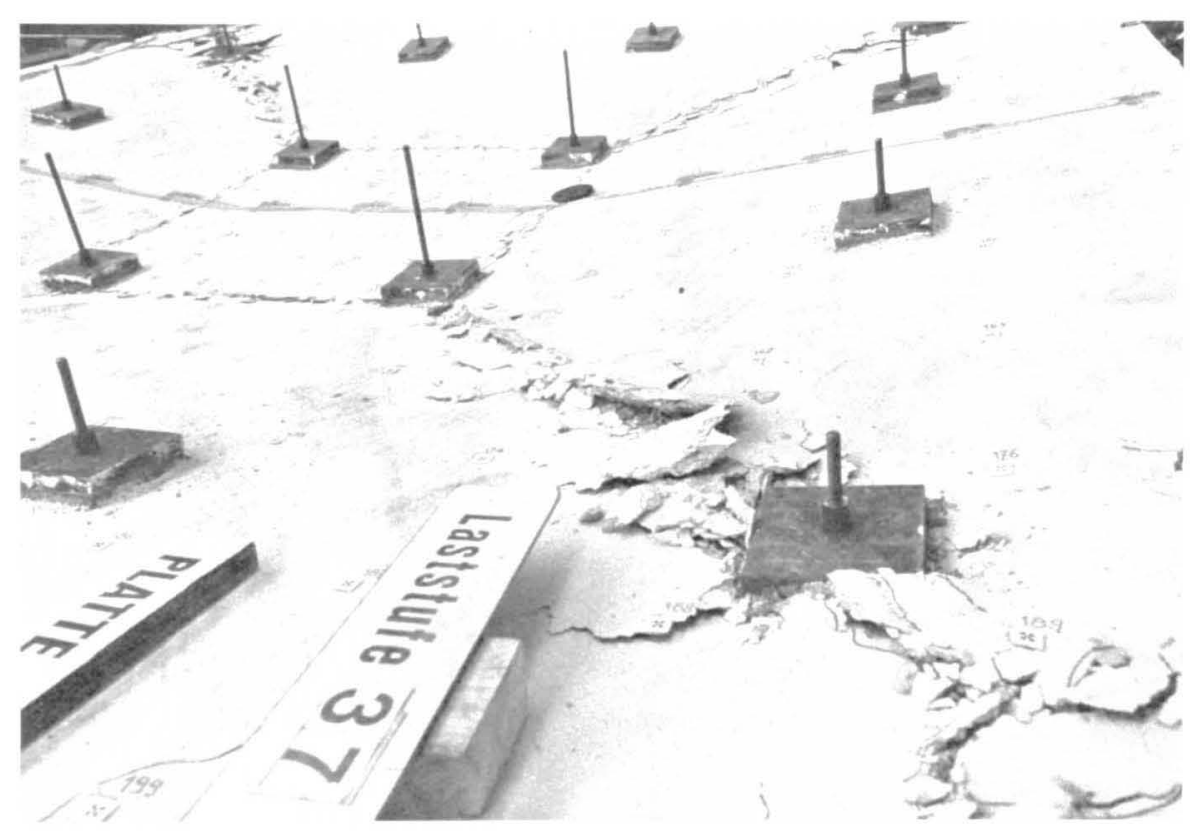

Bild 29: Bruchmechanismus Platte P1 (Oberseite) 


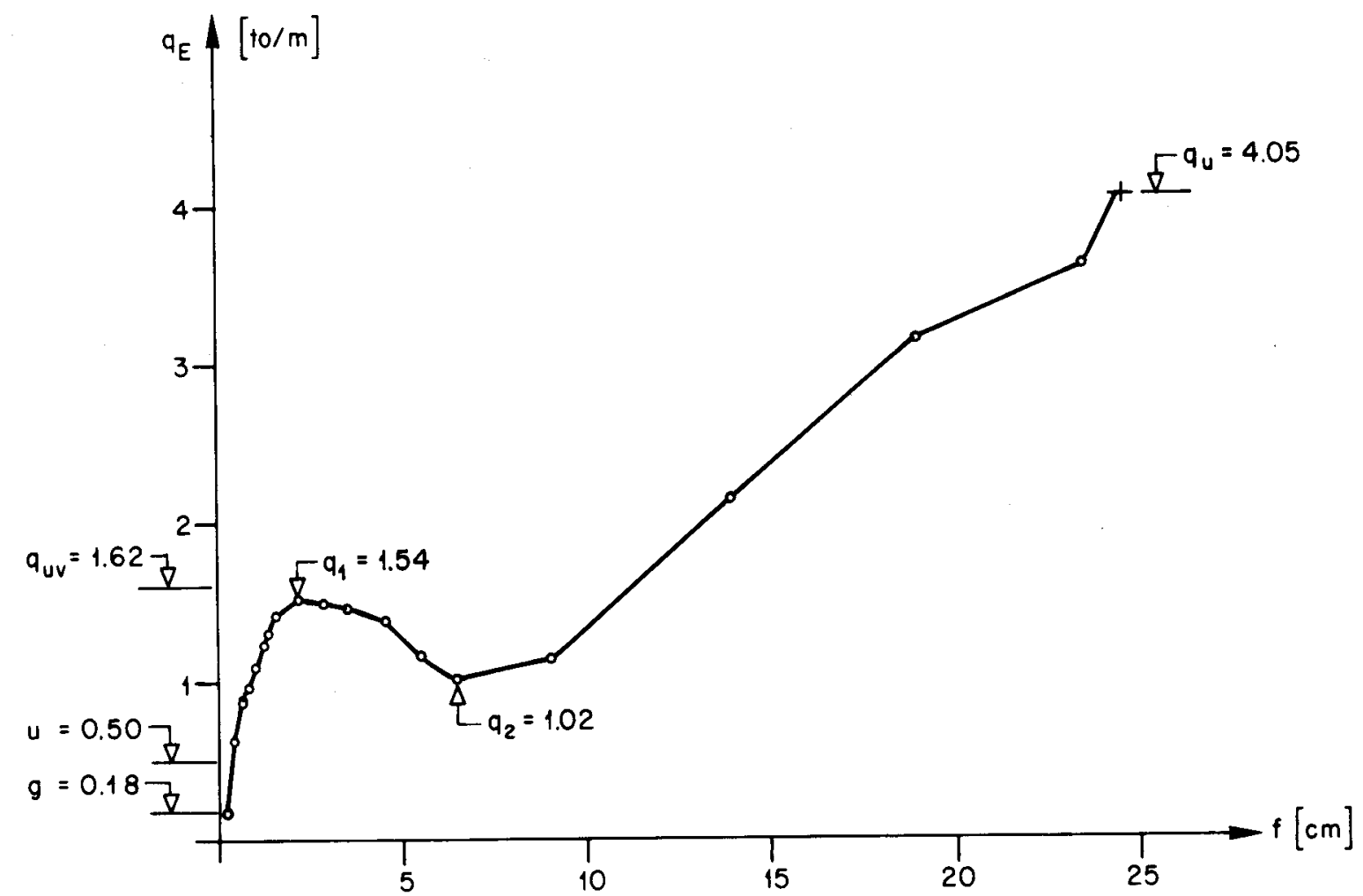

Bild 30 : Belastung Plattenstreifen PS 1 in Funktion der Mitten = durchbiegung

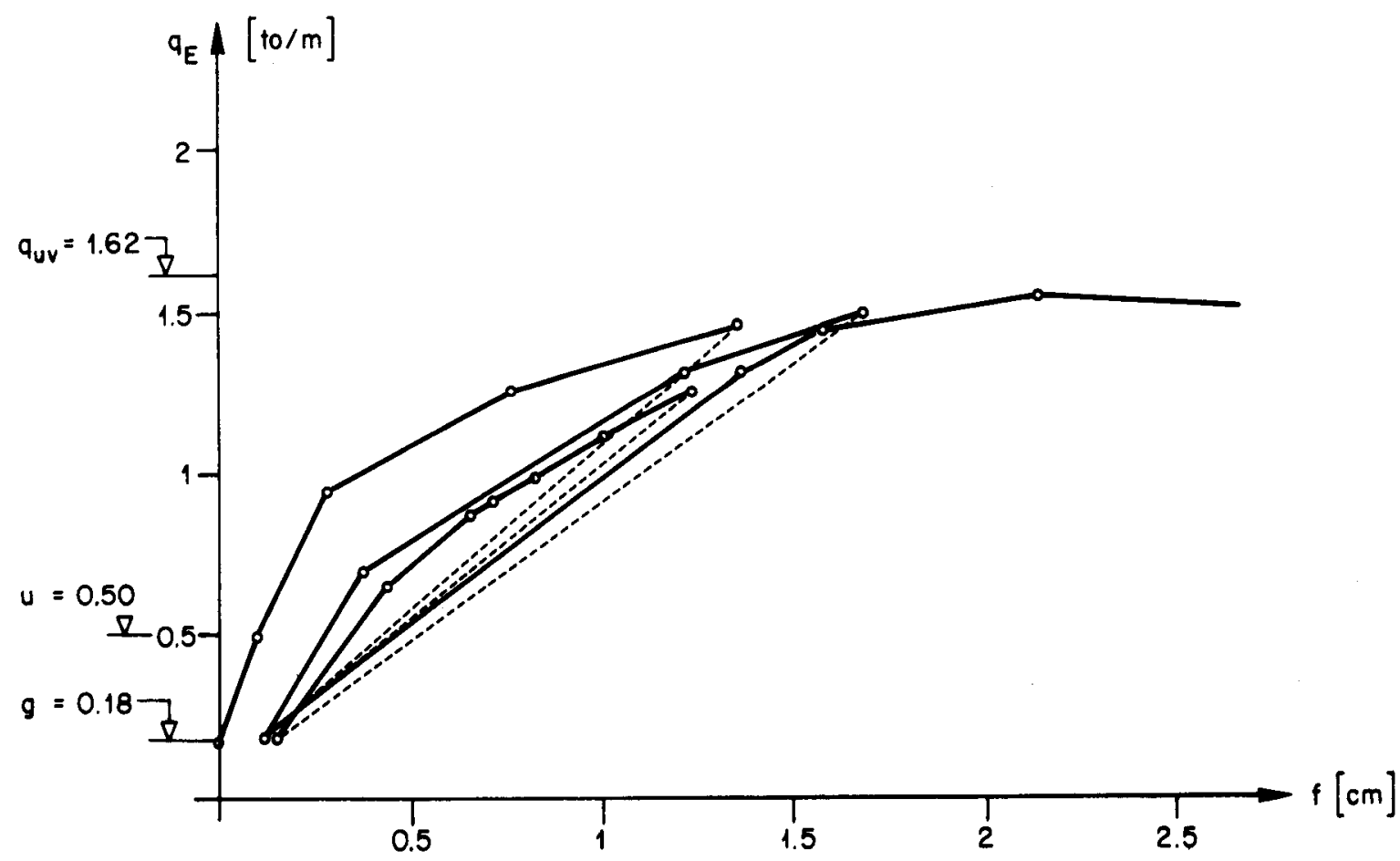

Bild 31 : Belastung Plattenstreifen PS 1 in Funktion der Mittendurch = biegung (Anfongsbereich) 


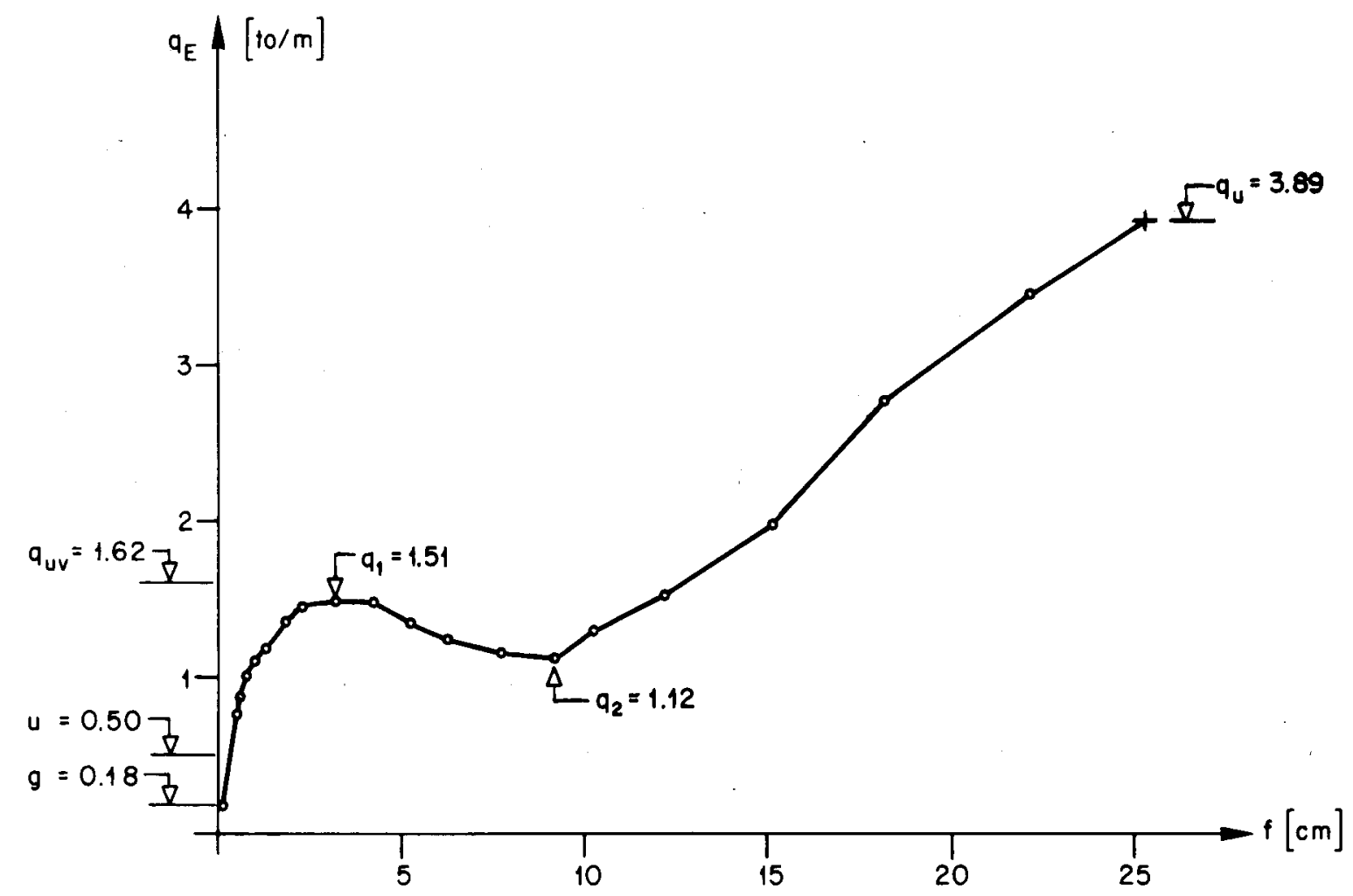

Bild 32 : Belastung Plattenstreifen PS 2 in. Funktion der Mitten= durchbiegung

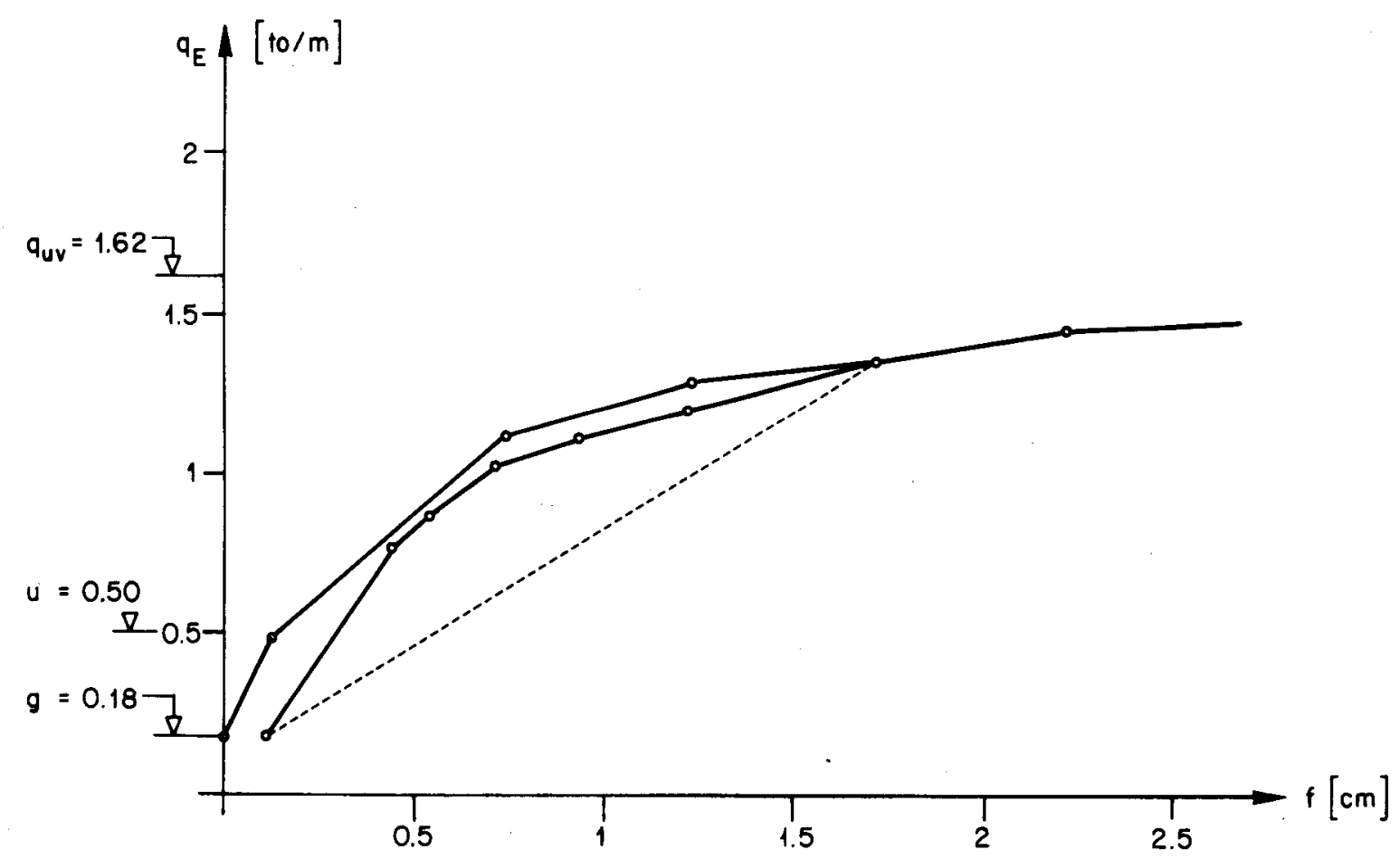

Bild 33 : Belastung Plattenstreifen PS 2 in Funktion der Mittendurch = biegung (Anfangsbereich) 


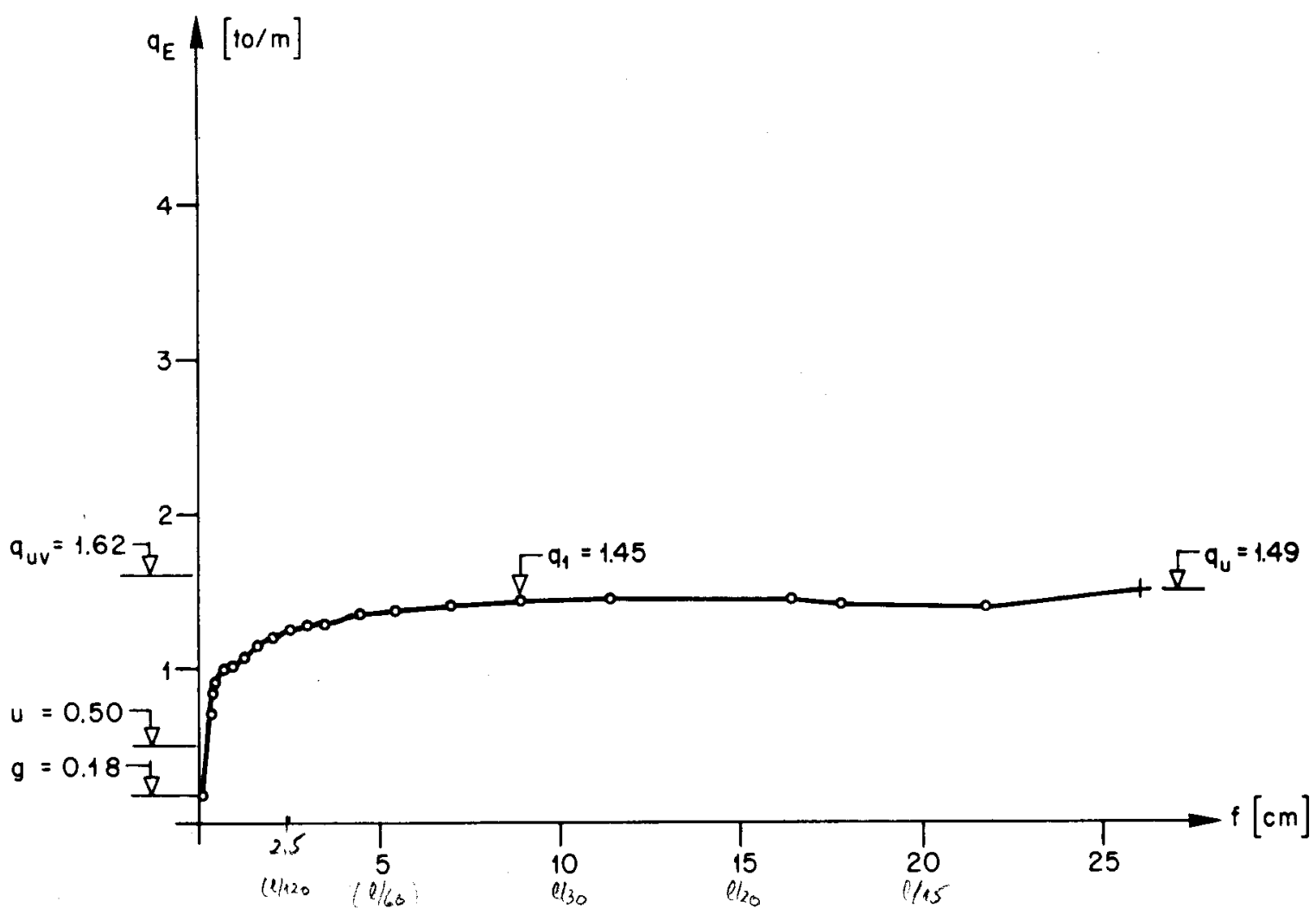

Bild 34 : Belastung Plattenstreifen PS 3 in Funktion der Mitten = durchbiegung

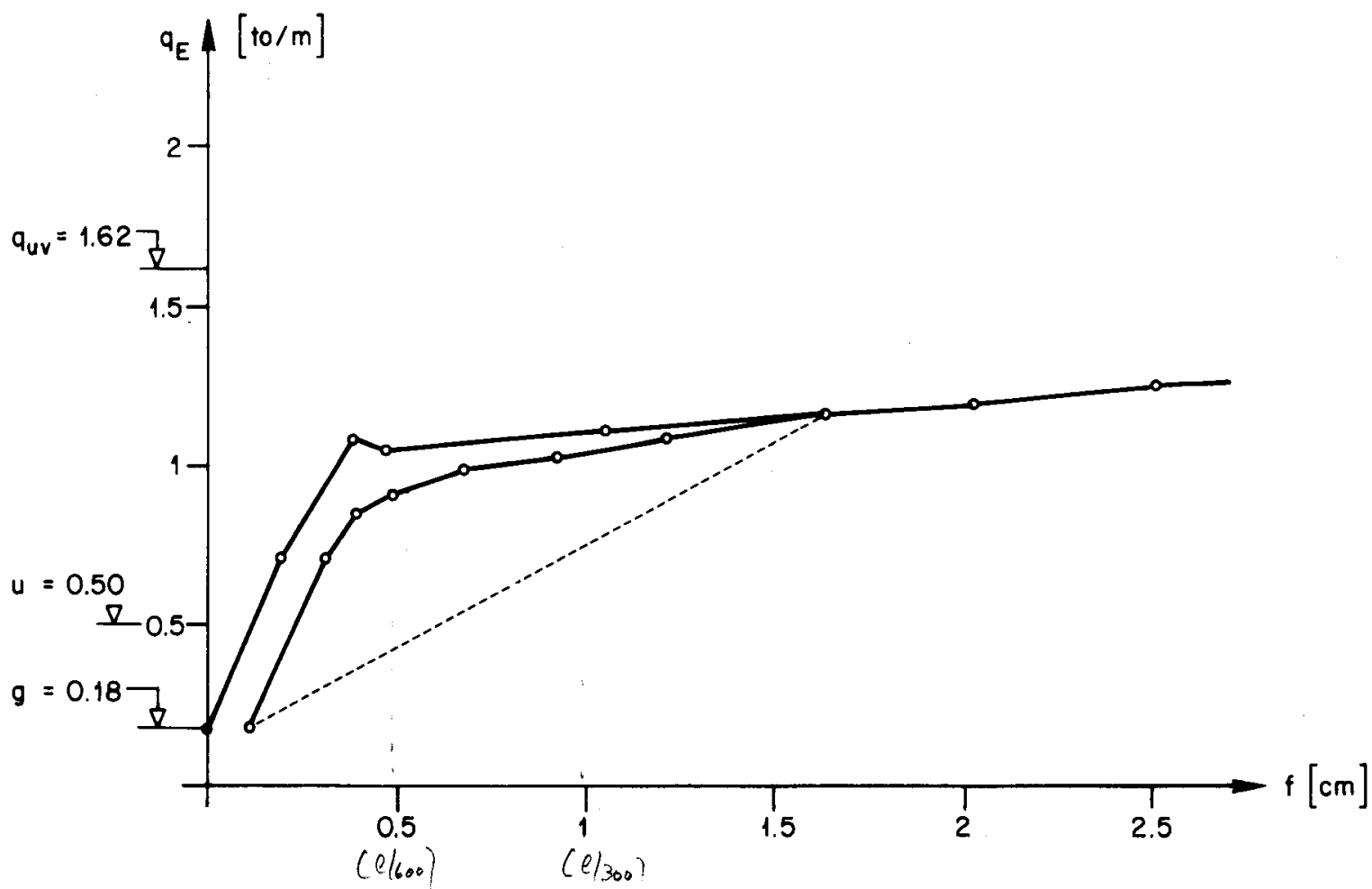

Bild 35 : Belastung Plattenstreifen PS 3 in Funktion der Mittendurch = biegung (Anfongsbereich) 


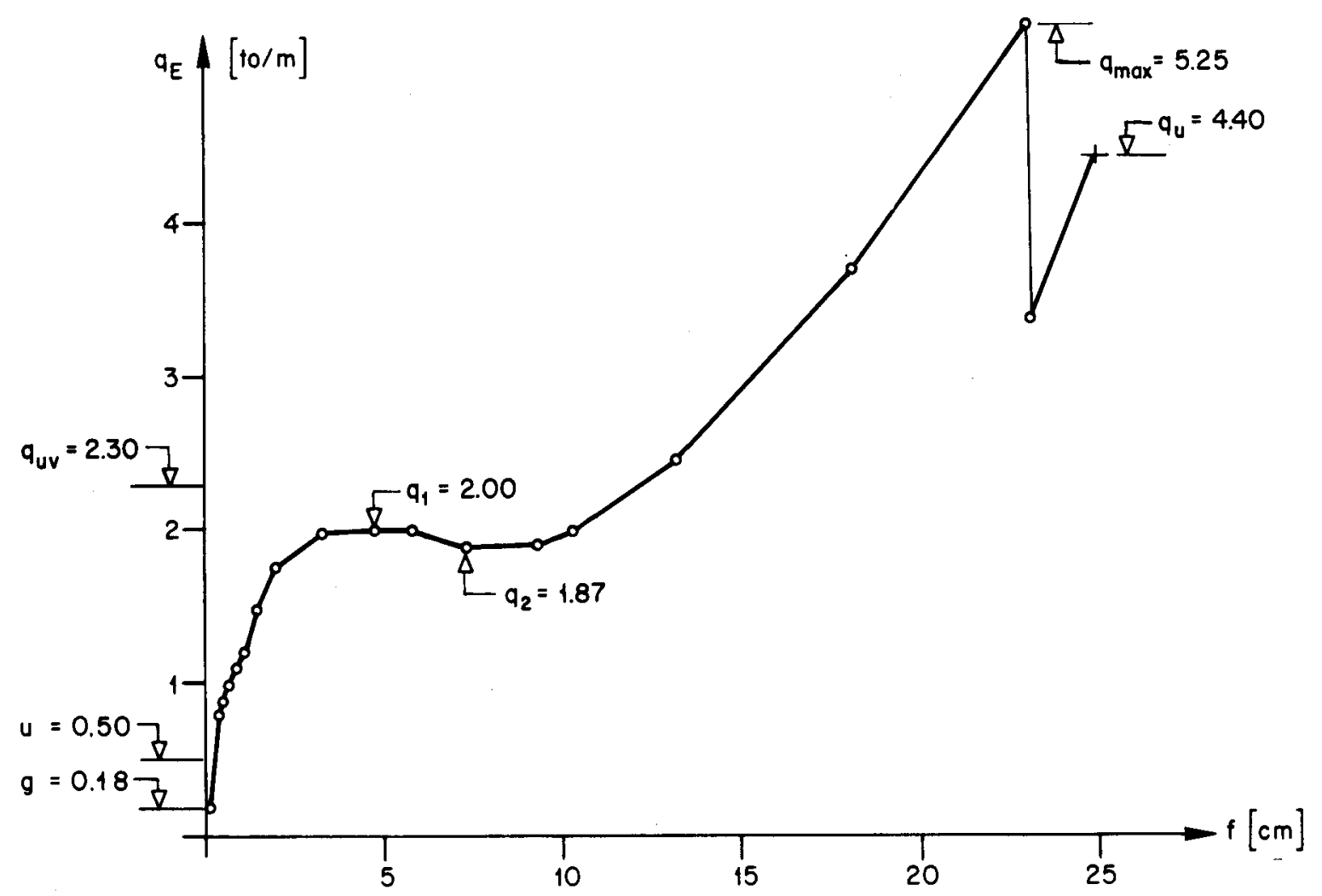

Bild 36 : Belastung Plattenstreifen PS 4 in Funktion der Mitten = durchbiegung

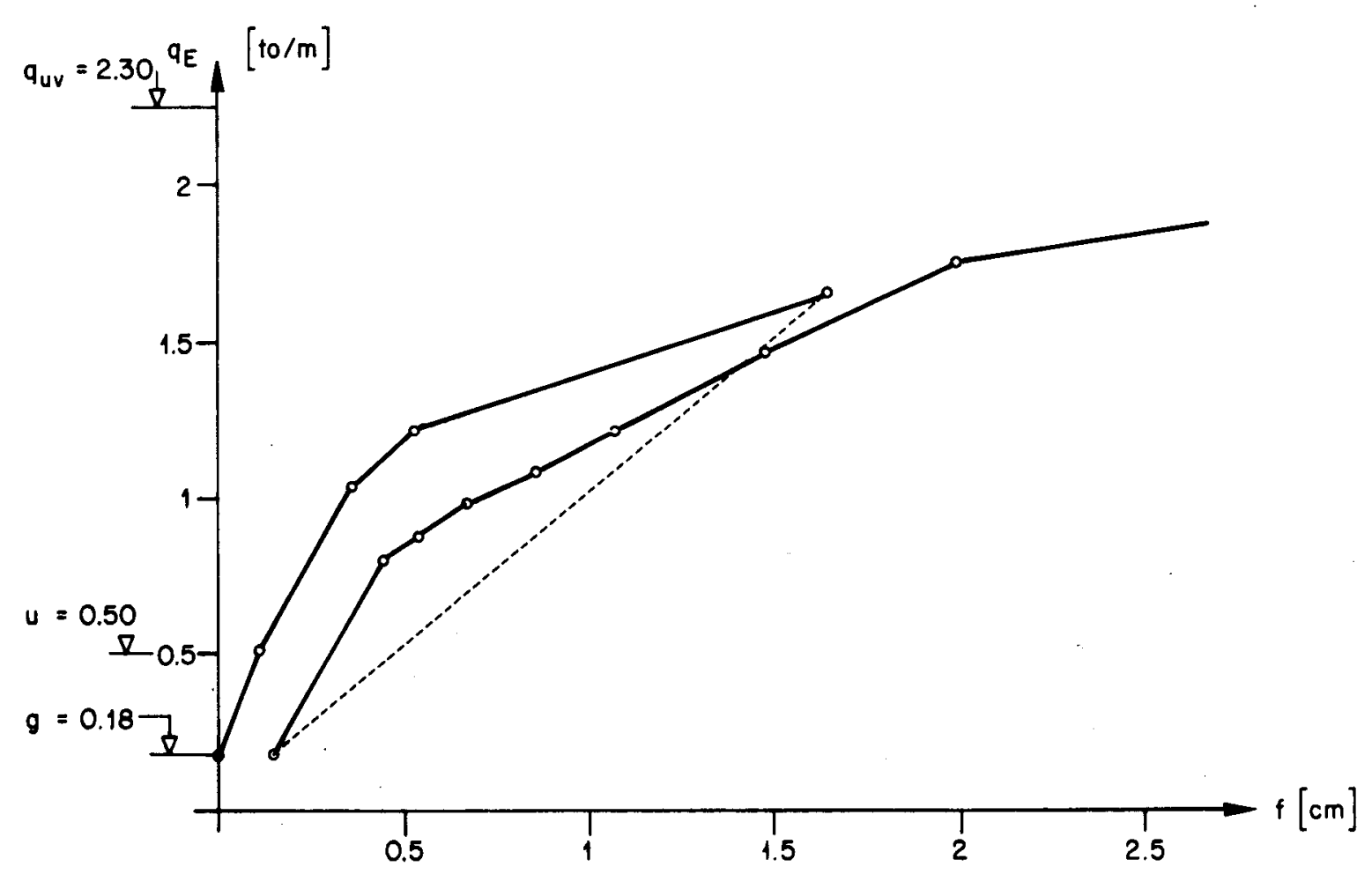

Bild 37 : Belastung Plattenstreifen PS 4 in Funktion der Mittendurch = biegung (Anfangsbereich) 


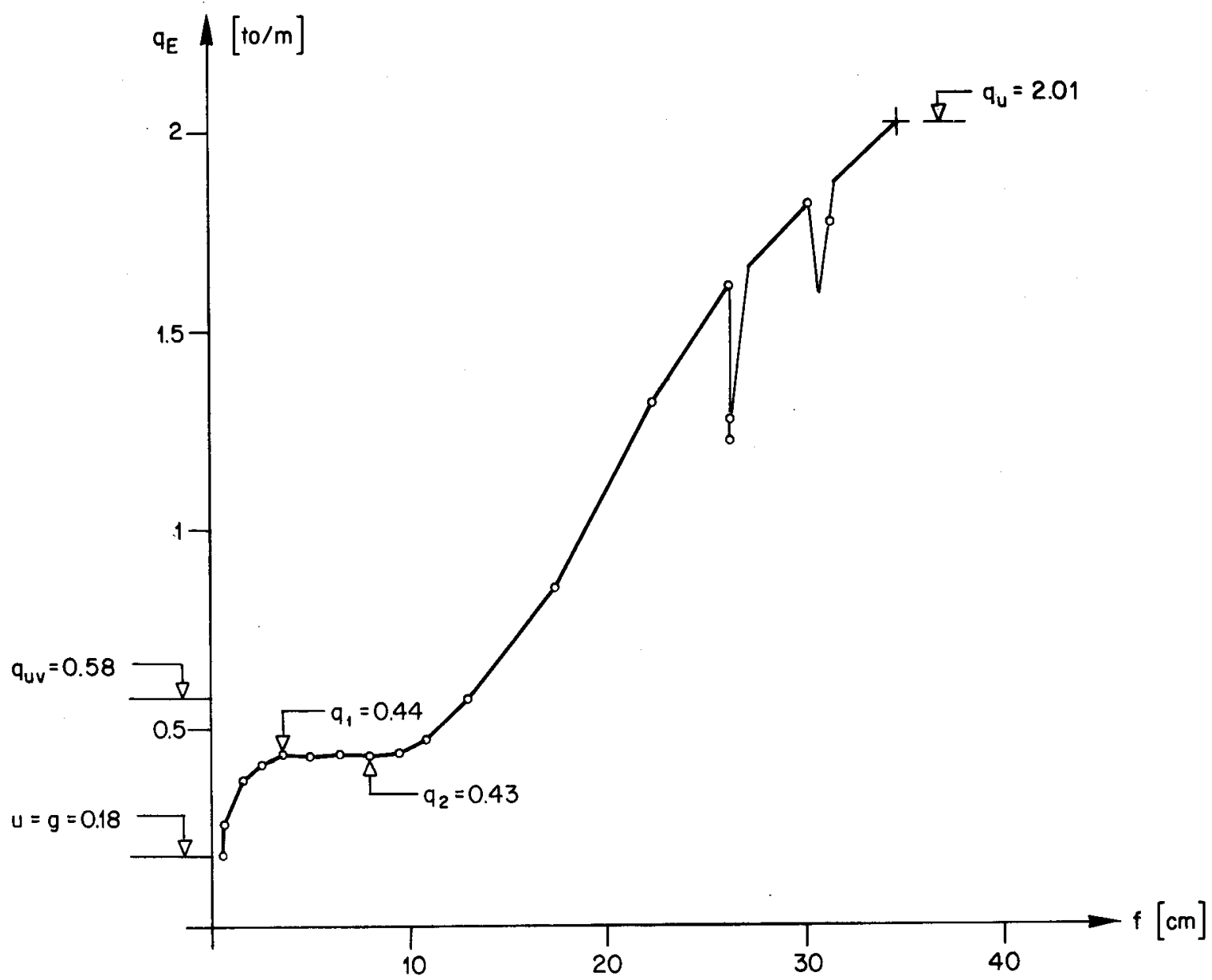

Bild 38: Belastung Plattenstreifen PS 5 in Funktion der Mittendurch = biegung

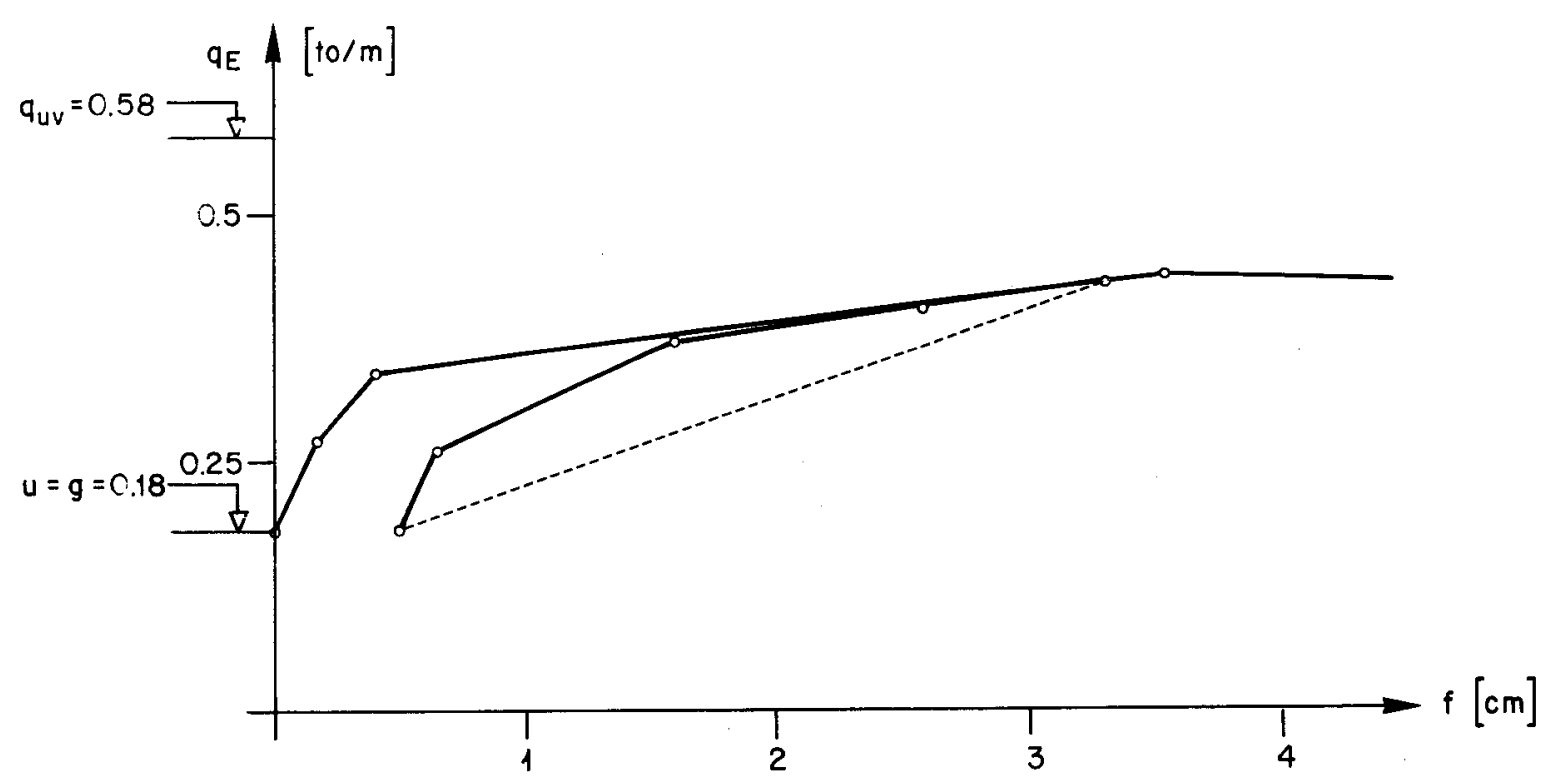

Bild 39: Belastung Plattenstreifen PS 5 in Funktion der Mittendurch= biegung (Anfangsbereich) 


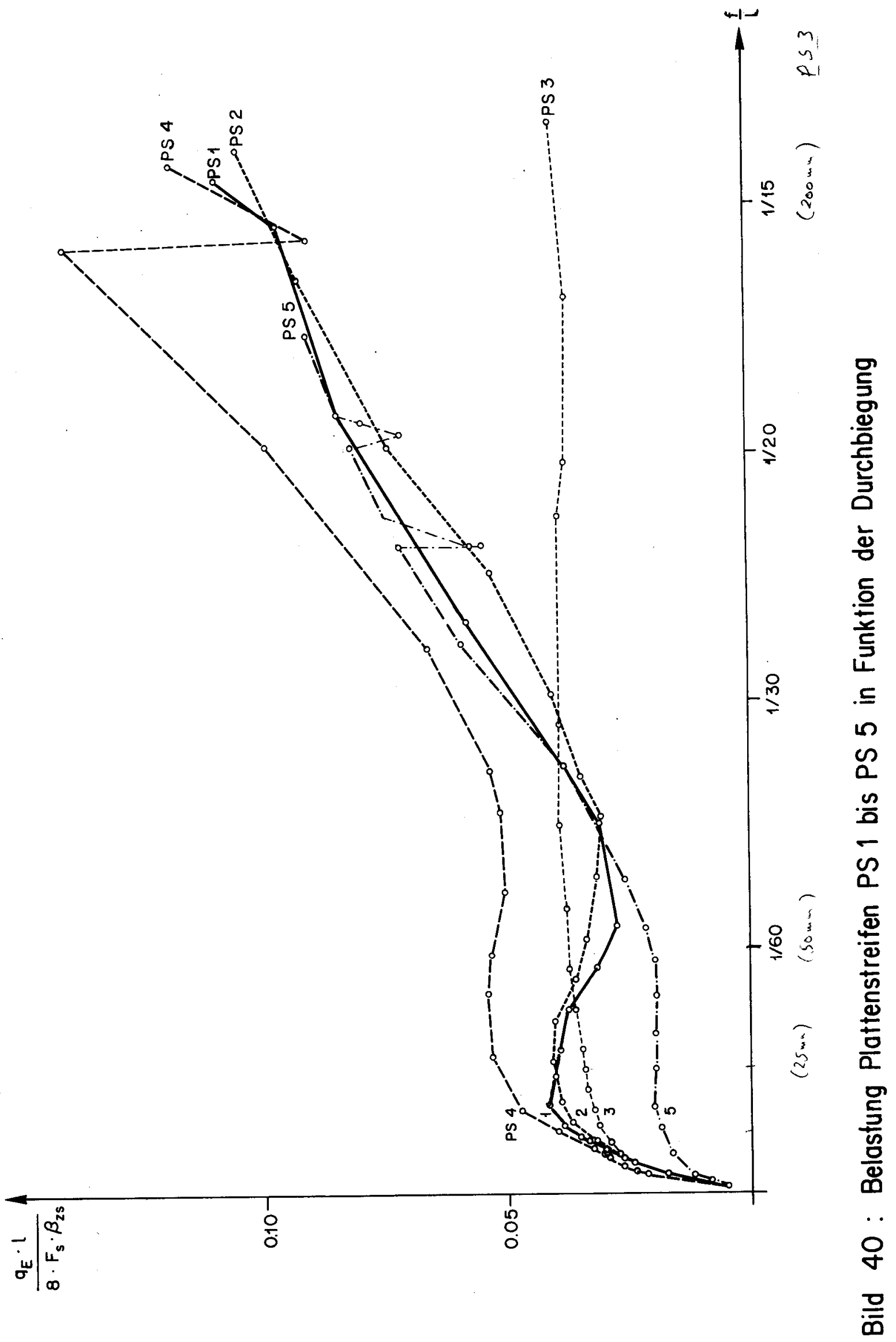




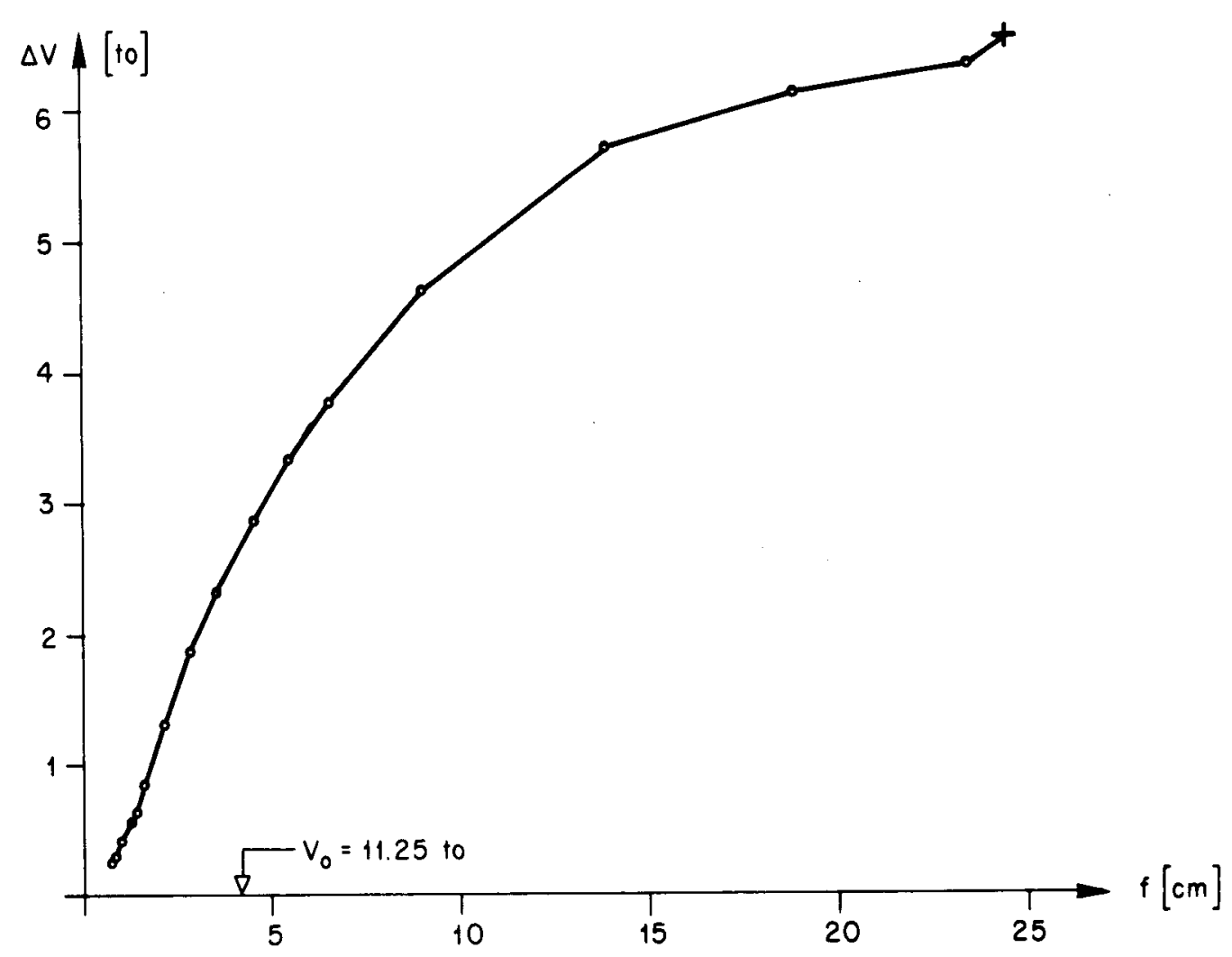

Bild 41: Kabelkraftzuwachs Plattenstreifen PS 1 in Funktion der Mitten = durchbiegung

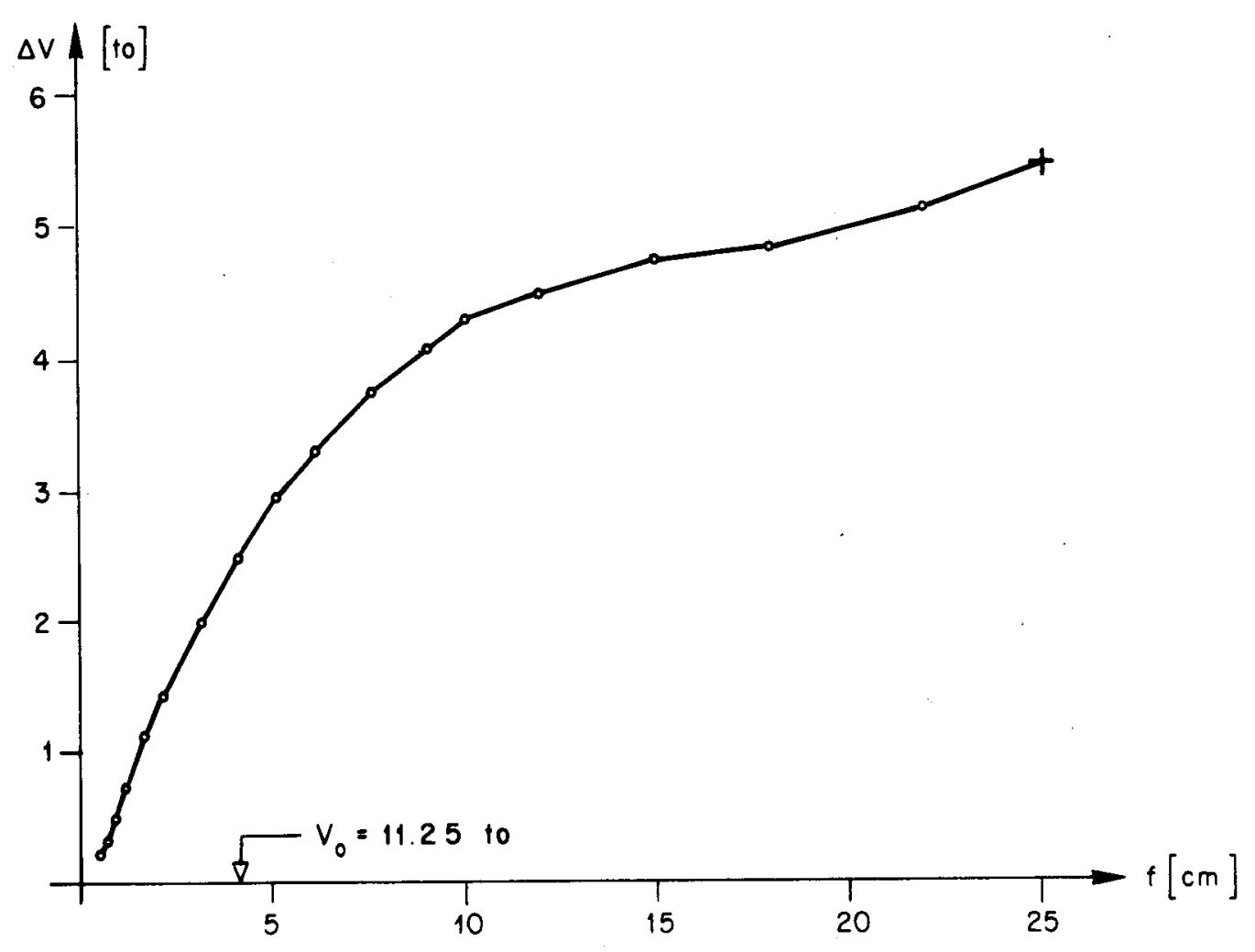

Bild 42: Kabelkraftzuwachs Plattenstreifen PS 2 in Funktion der Mitten = durchbiegung 


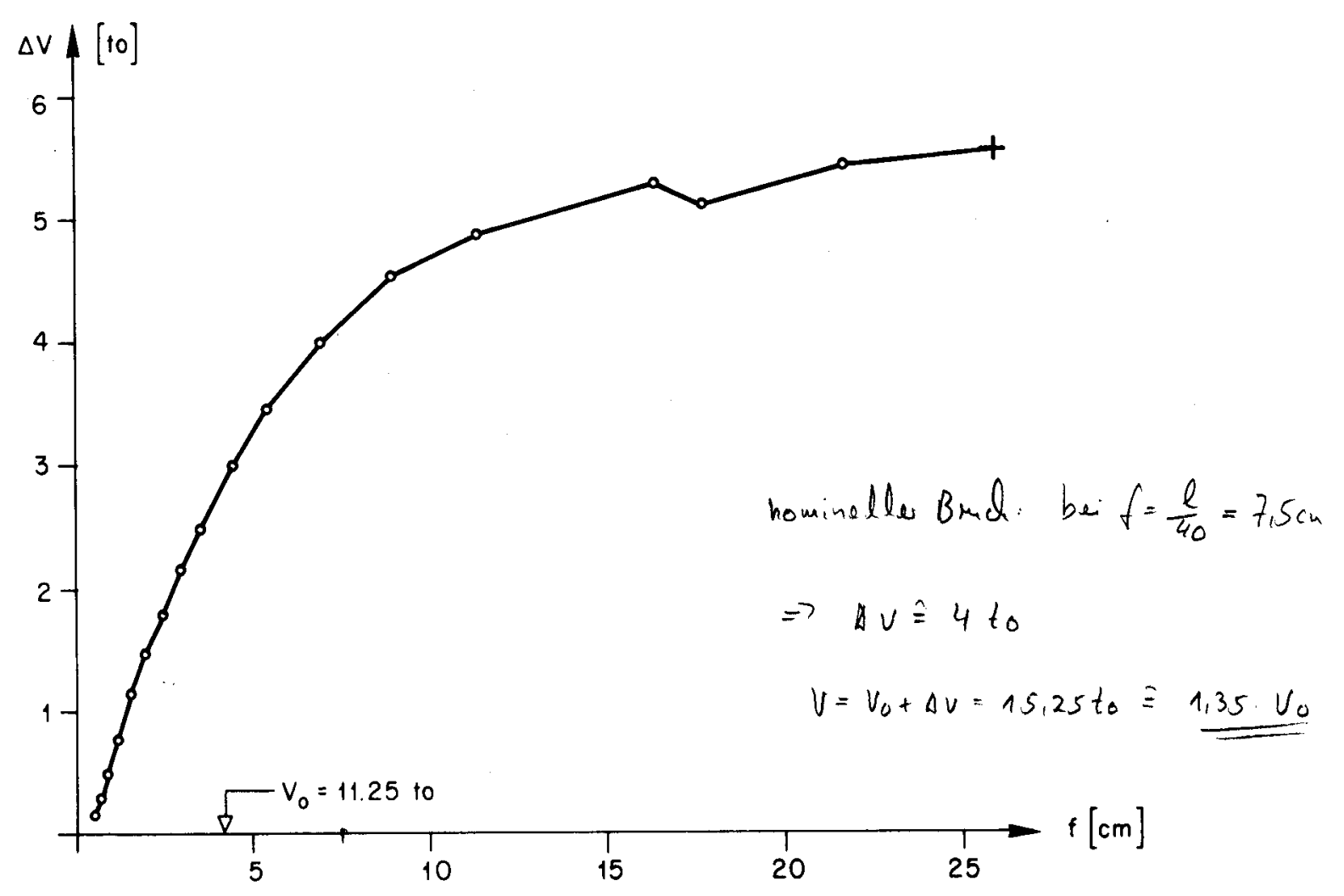

Bild 43: Kabelkraftzuwachs Plattenstreifen PS 3 in Funktion der Mitten $=$ durchbiegung (kine honzoulale stiking)

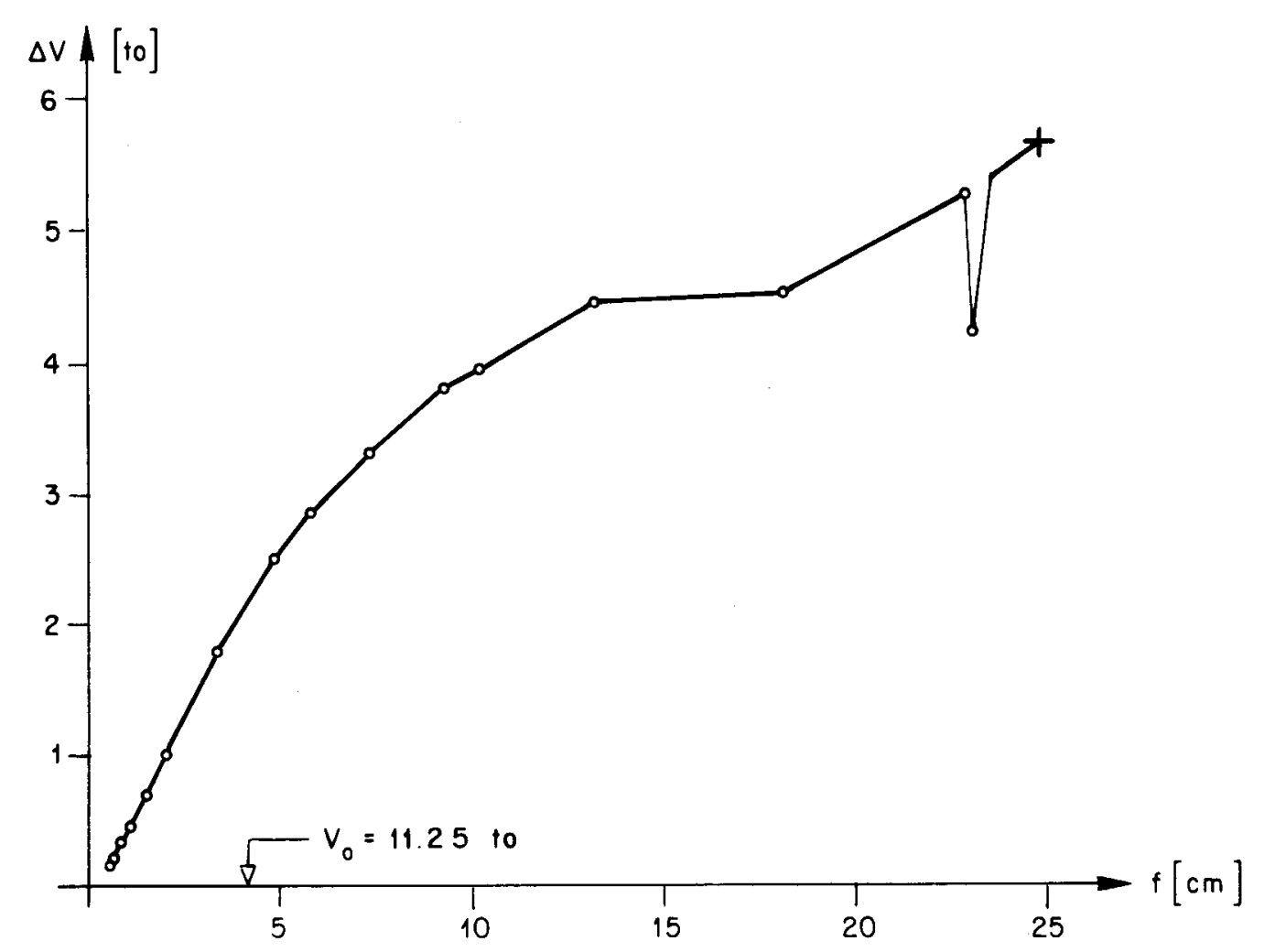

Bild 44: Kabelkraftzuwachs Plattenstreifen PS 4 in Funktion der Mitten = durchbiegung 


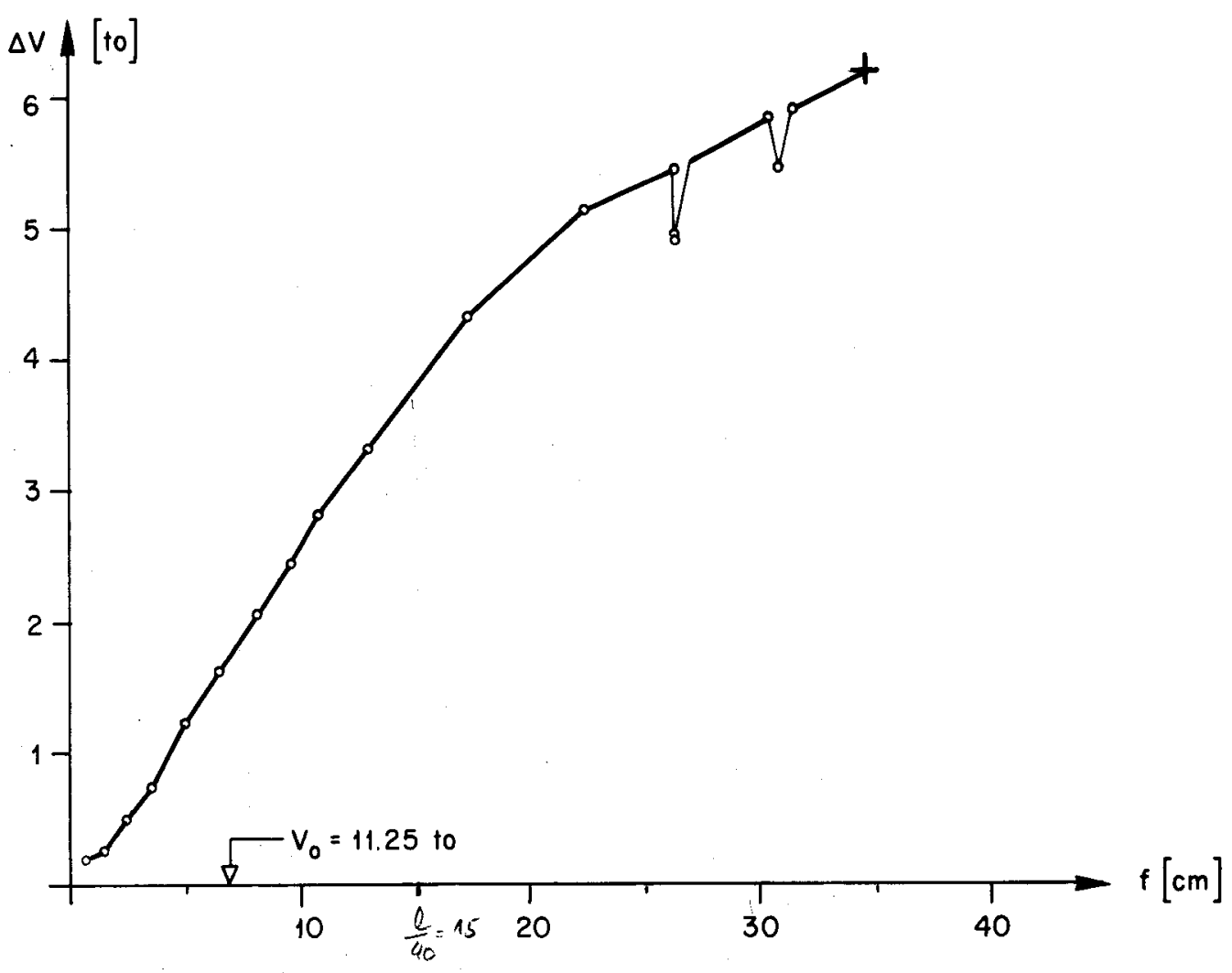

Bild 45: Kabelkraftzuwachs Plattenstreifen PS 5 in Funktion der Mitten = durchbiegung

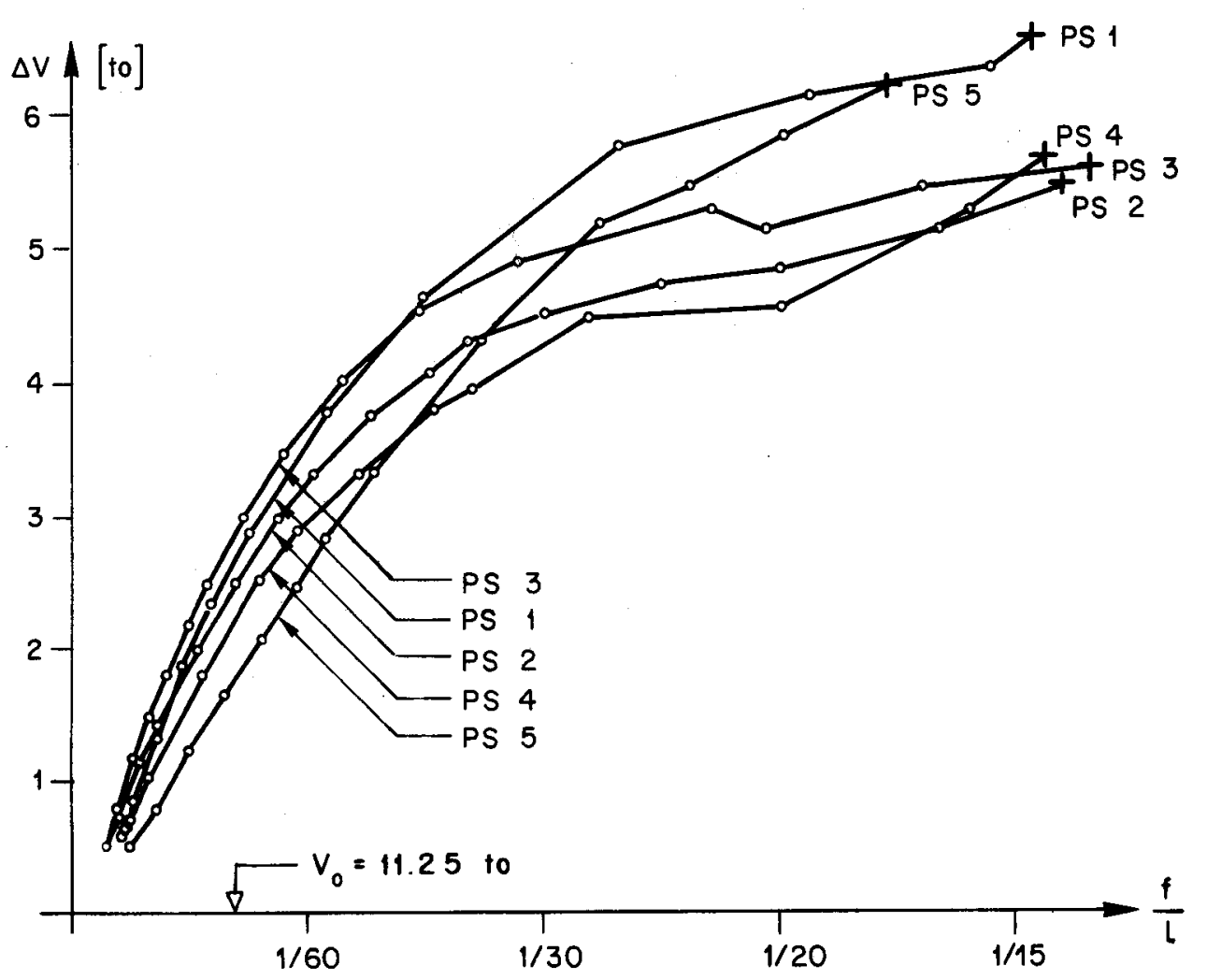

Bild 46: Kabelkraftzuwachs Plattenstreifen PS 1 bis PS 5 in Funktion der Mittendurchbiegung 


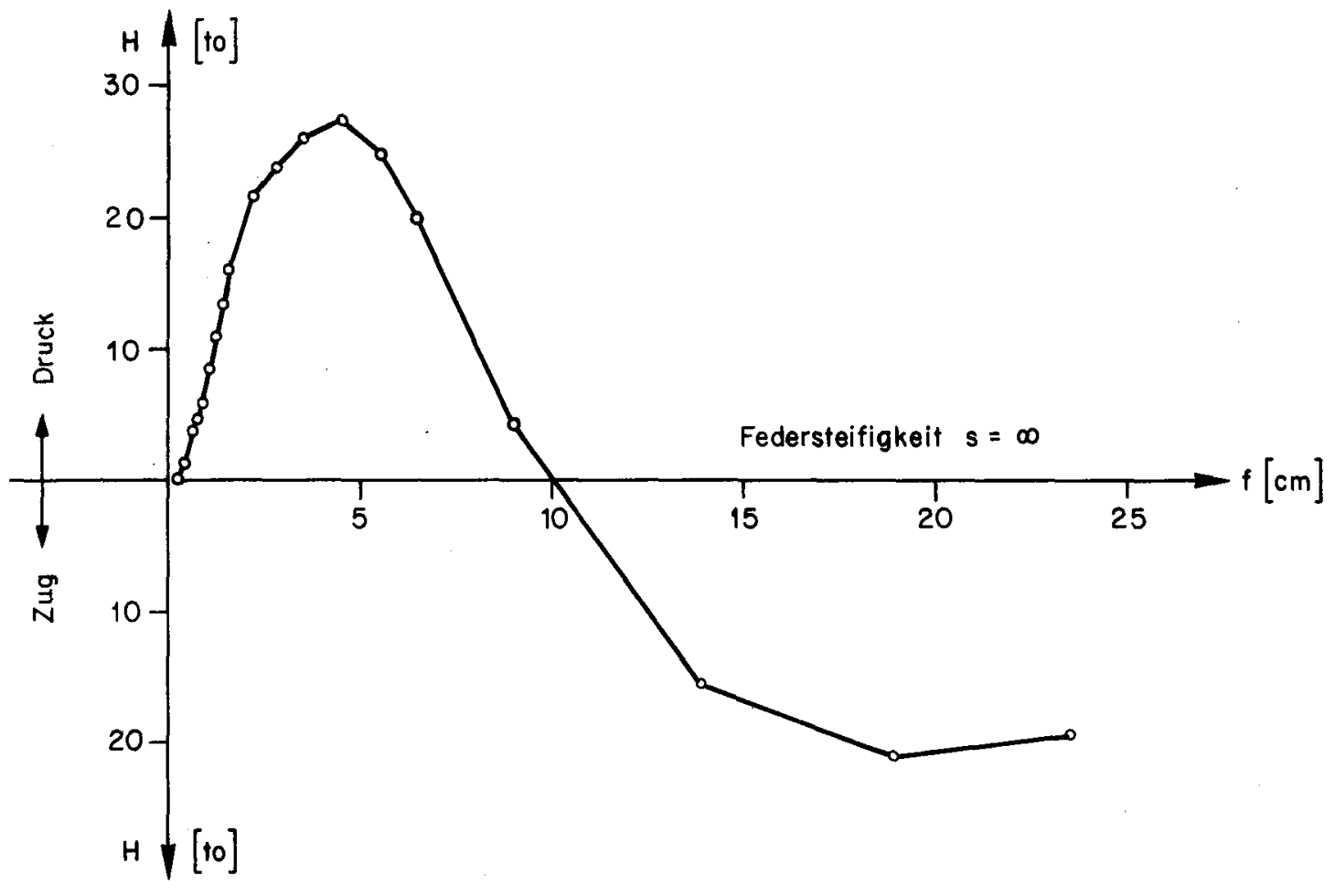

Bild 47: Horizontalkraft Plattenstreifen PS 1 in Funktion der Mittendurchbiegung

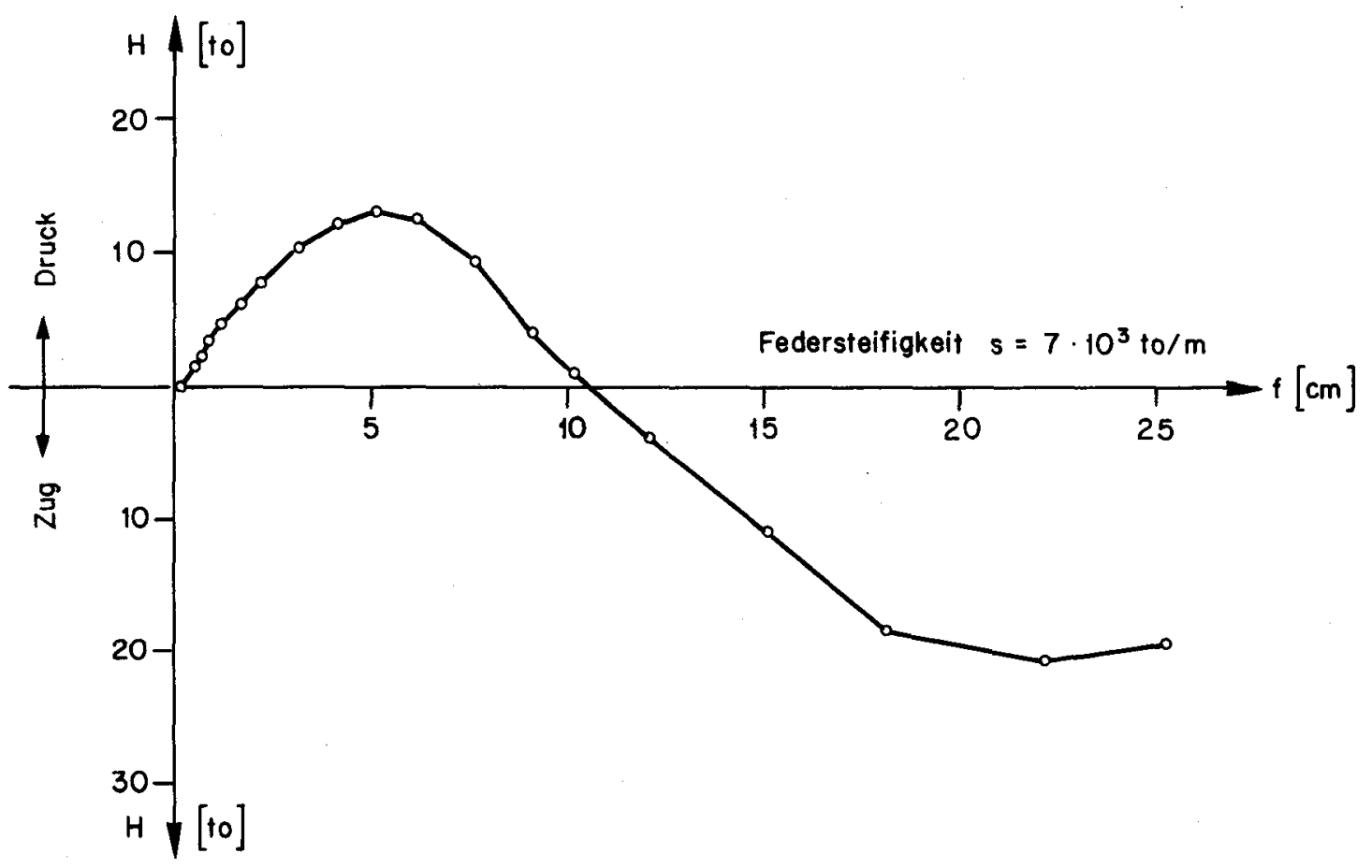

Bild 48: Horizontalkraft Plattenstreifen PS 2 in Funktion der Mittendurchbiegung 


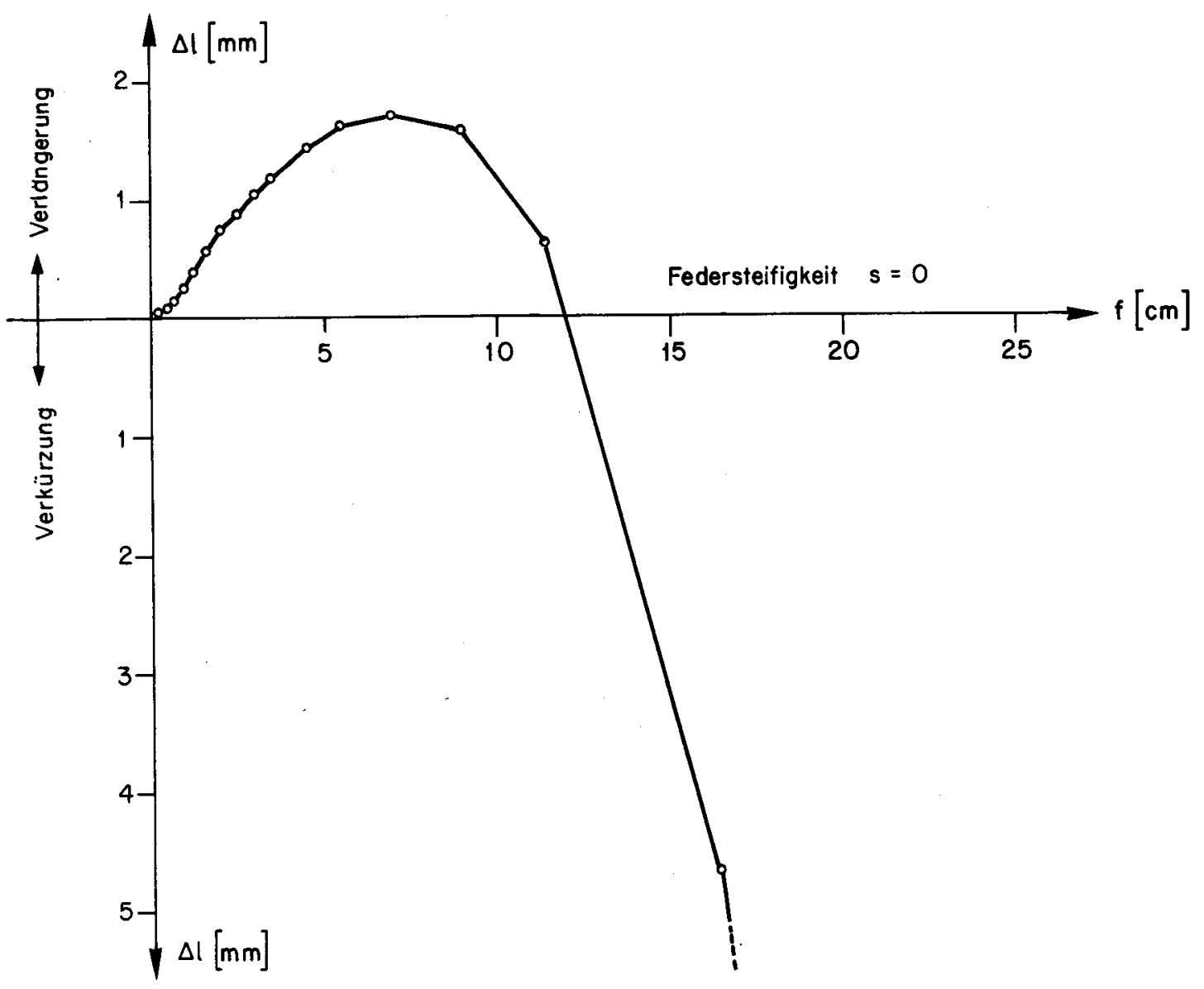

Bild 49: Längenänderung Plattenstreifen PS 3 in Funktion der Mittendurchbiegung

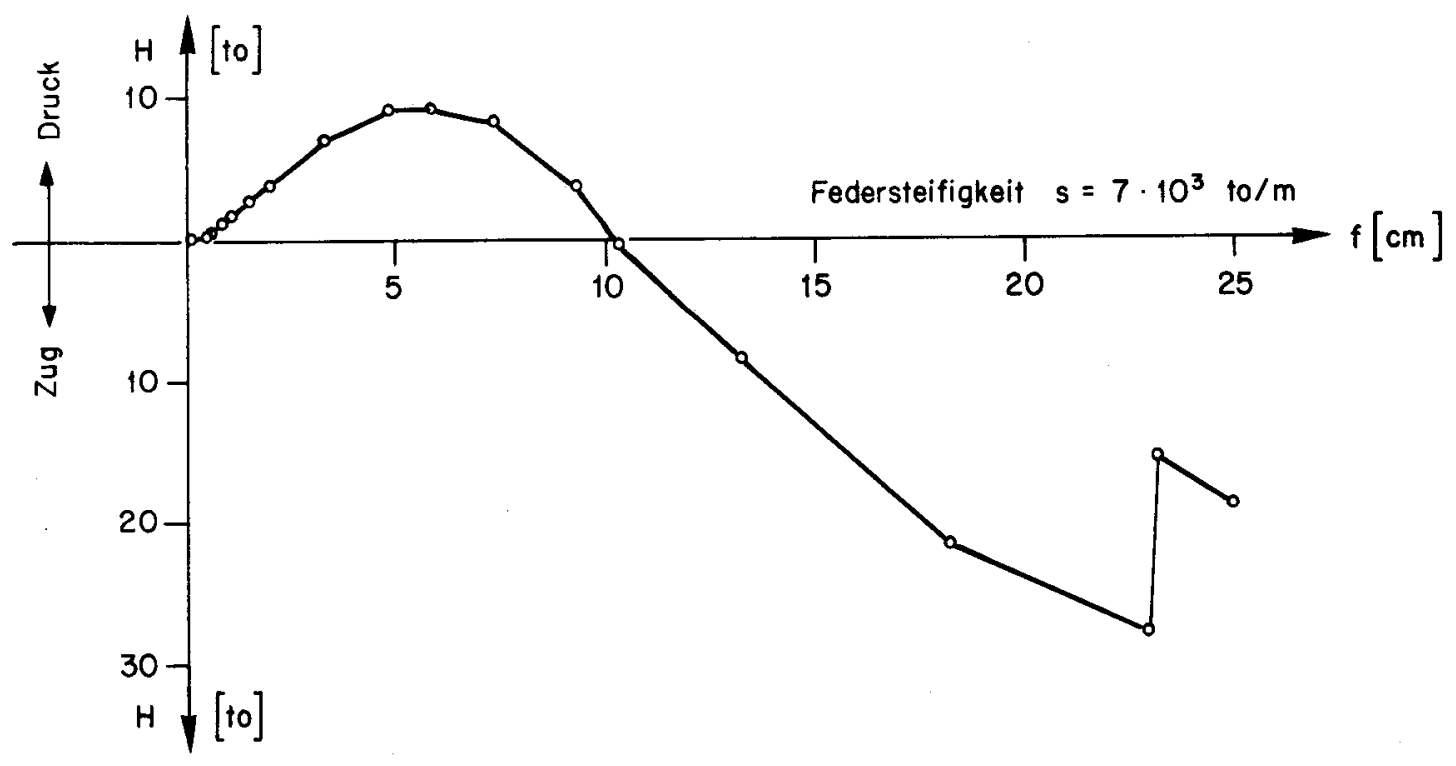

Bild 50: Horizontalkraft Plattenstreifen PS 4 in Funktion der Mittendurchbiegung 


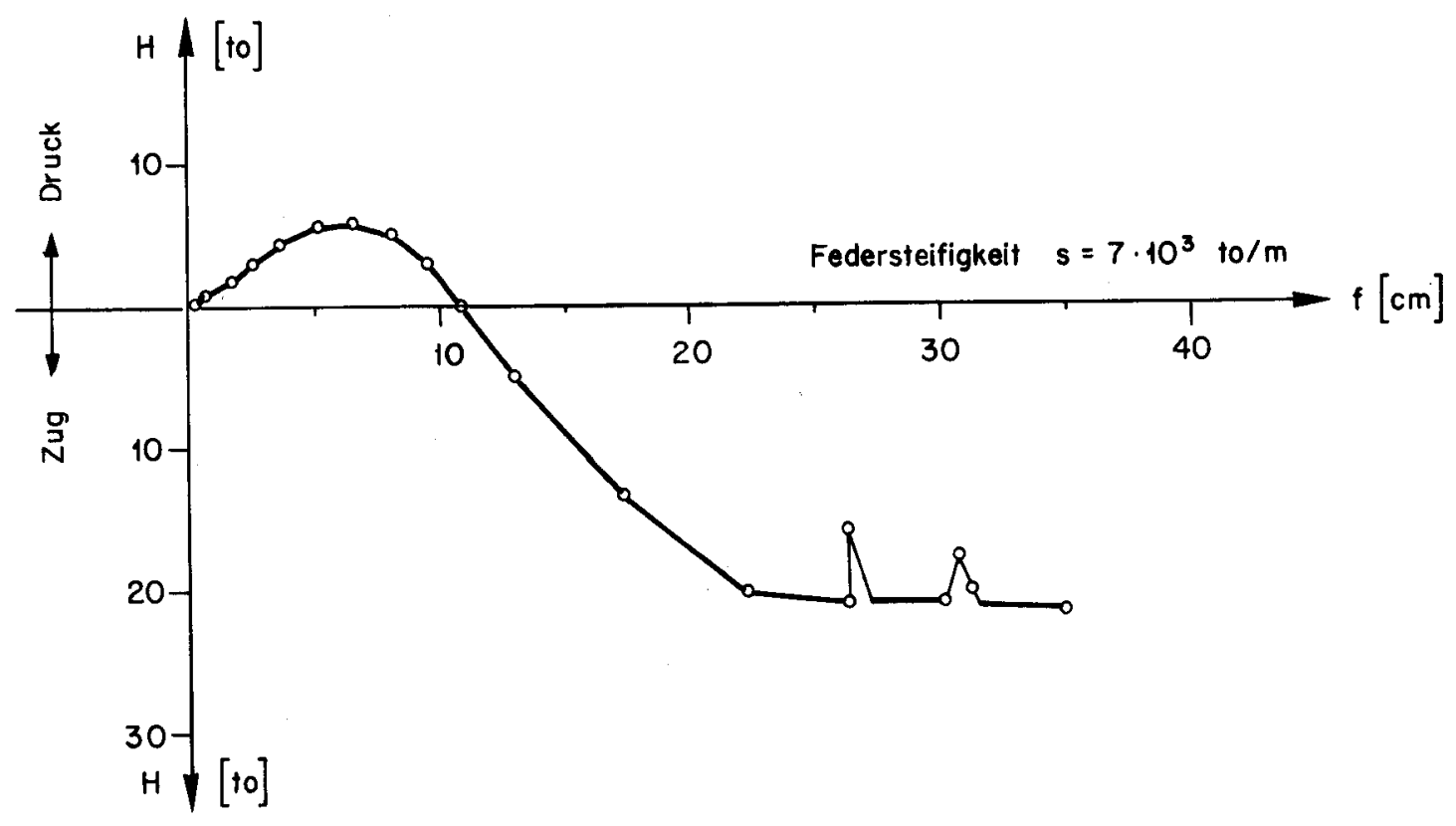

Bild 51: Horizontalkraft Plattenstreifen PS 5 in Funktion der Mittendurchbiegung

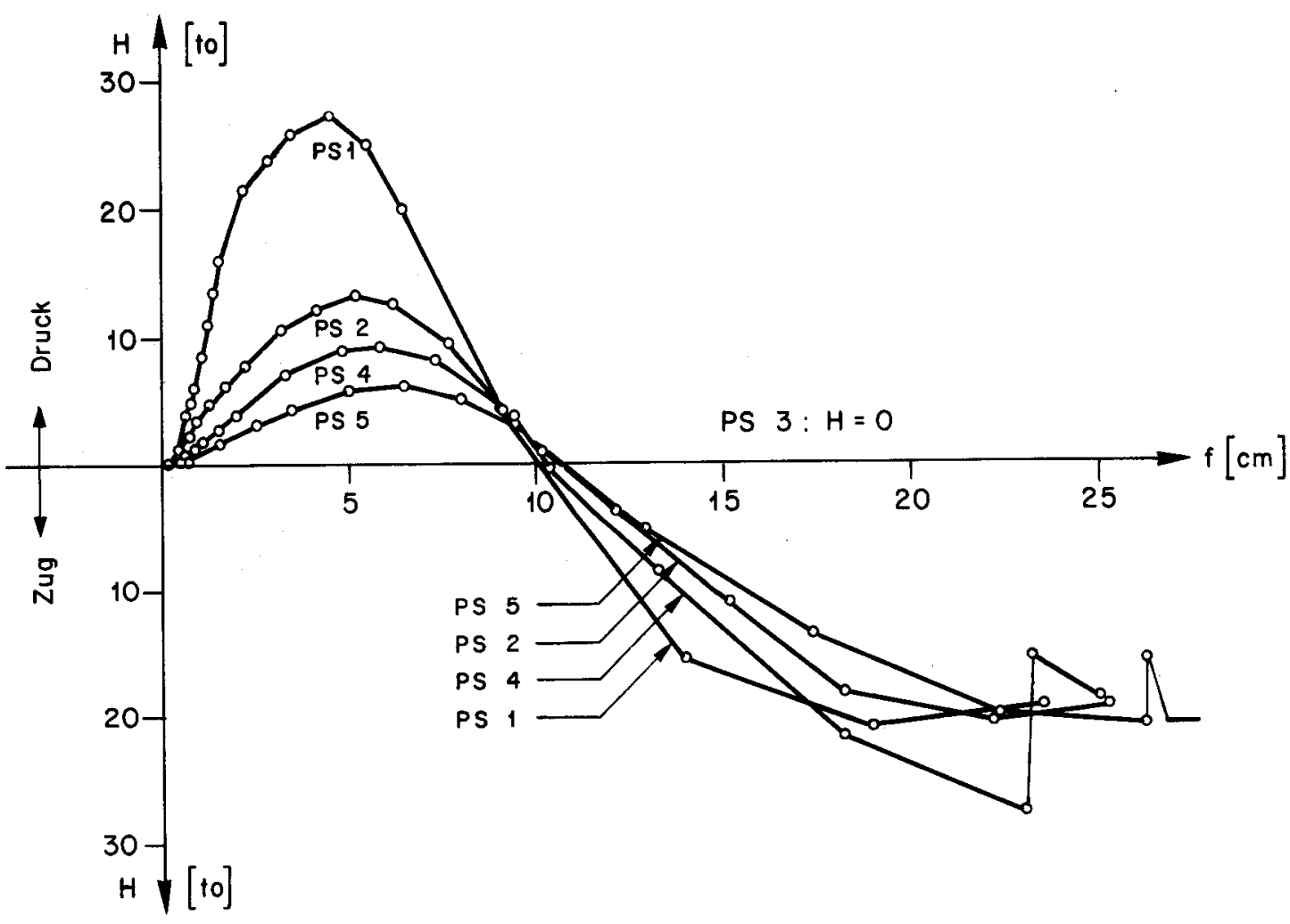

Bild 52: Horizontalkraft Plattenstreifen PS 1 bis PS 5 in Funktion der Mittendurchbiegung 

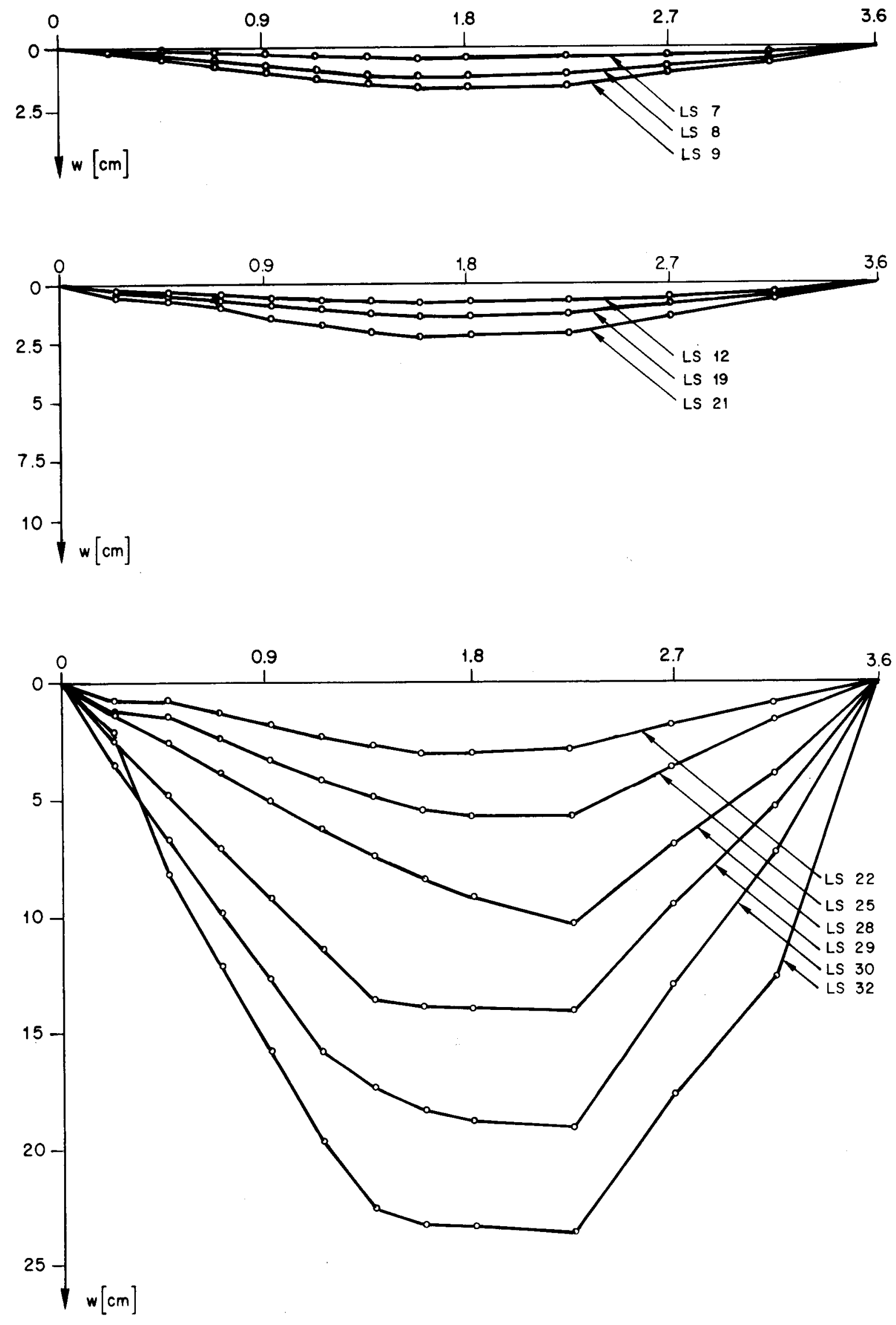

Bild 53: Verlauf der Durchbiegungen Plattenstreifen PS 1 

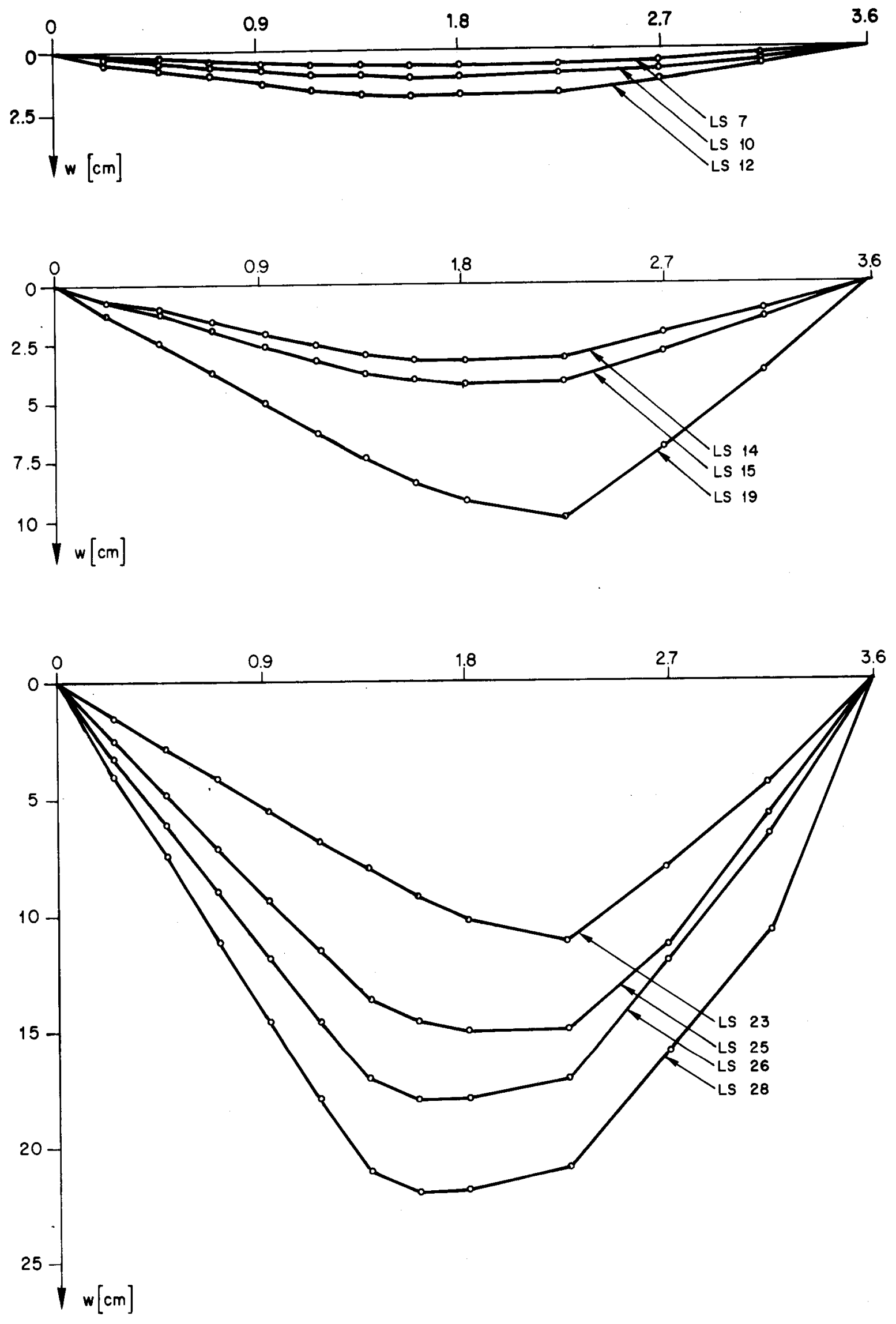

Bild 54: Verlauf der Durchbiegungen Plattenstreifen PS 2 

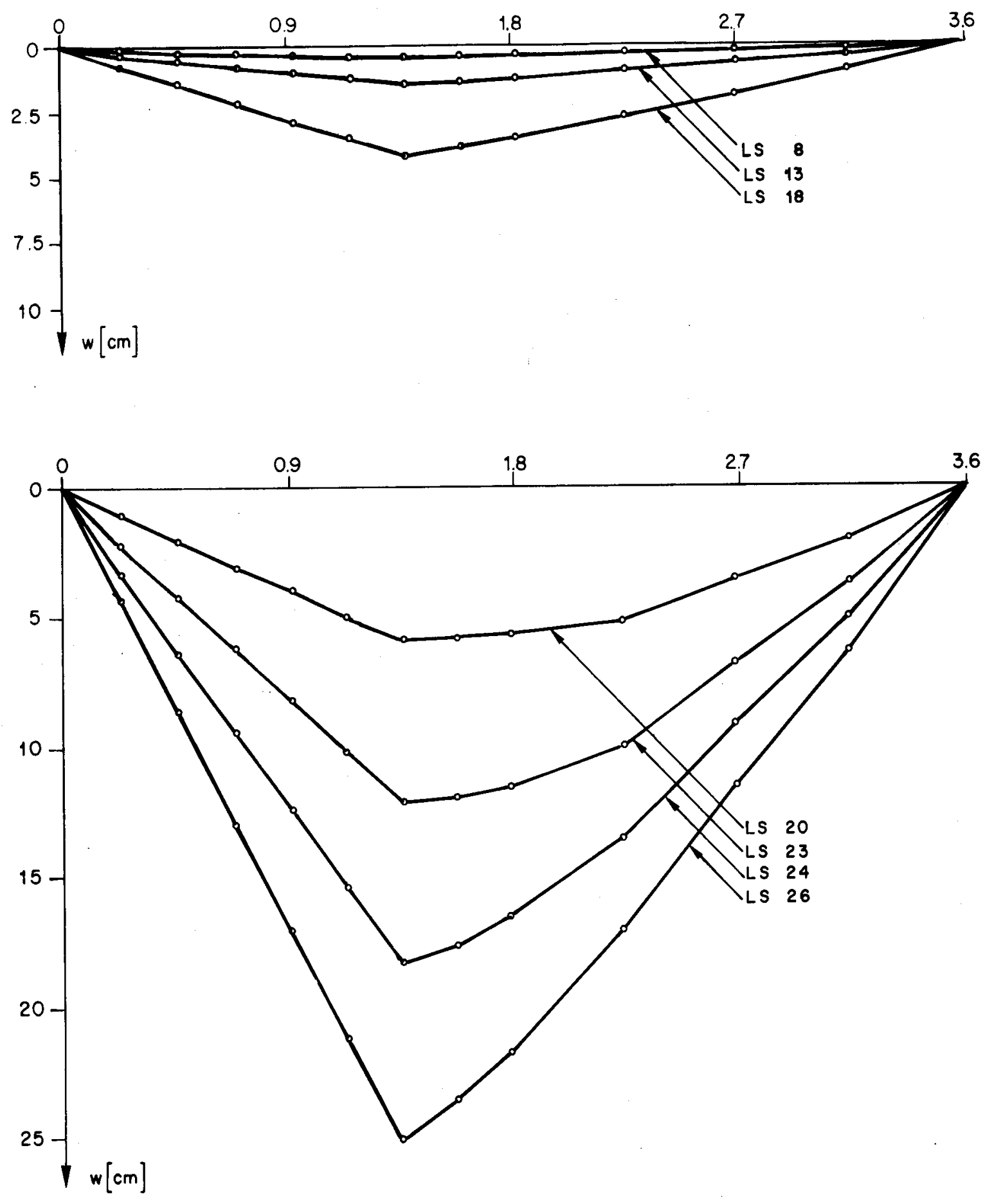

Bild 55: Verlauf der Durchbiegungen Plattenstreifen PS 3 

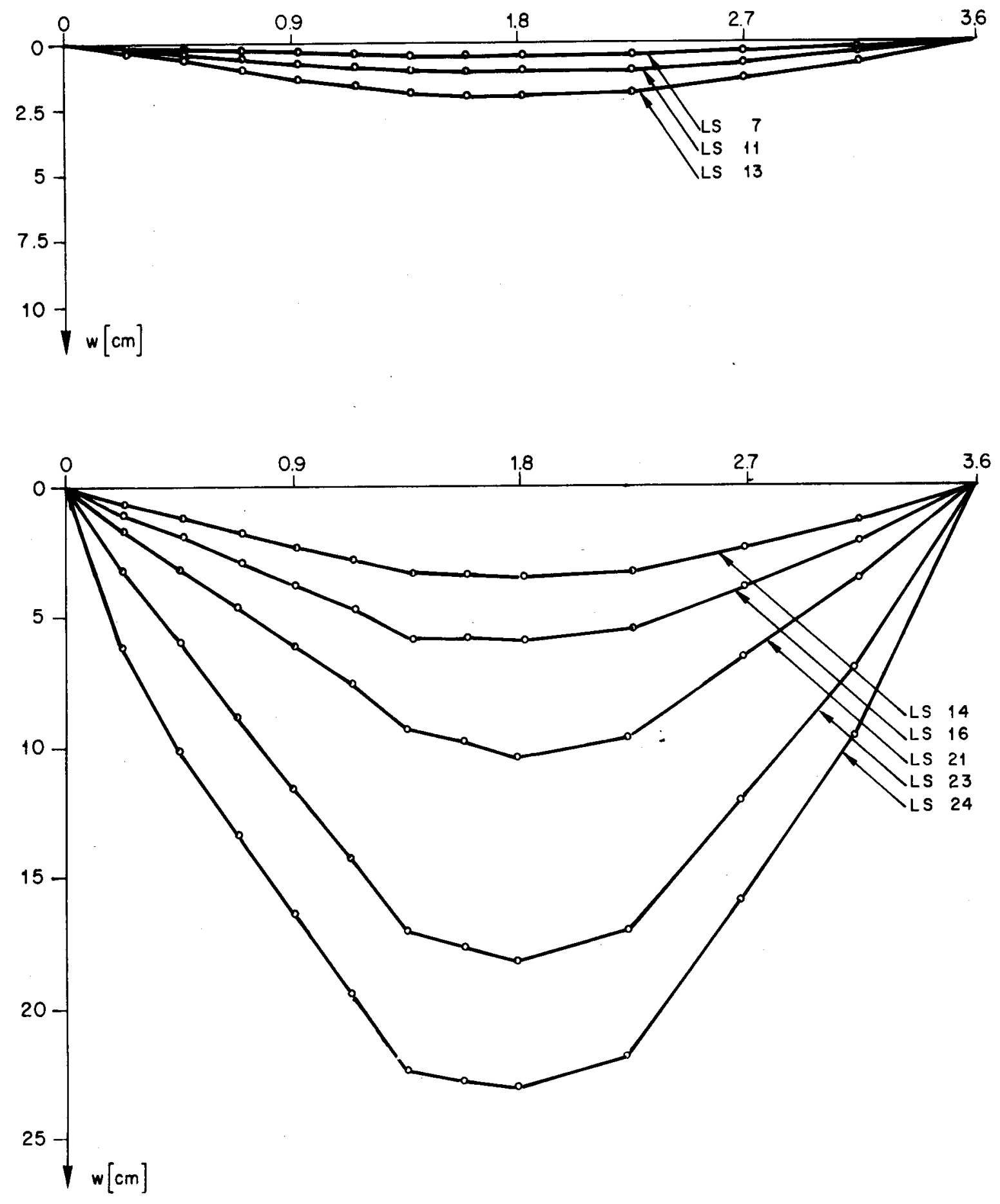

Bild 56: Verlauf der Durchbiegungen Plattenstreifen PS 4 

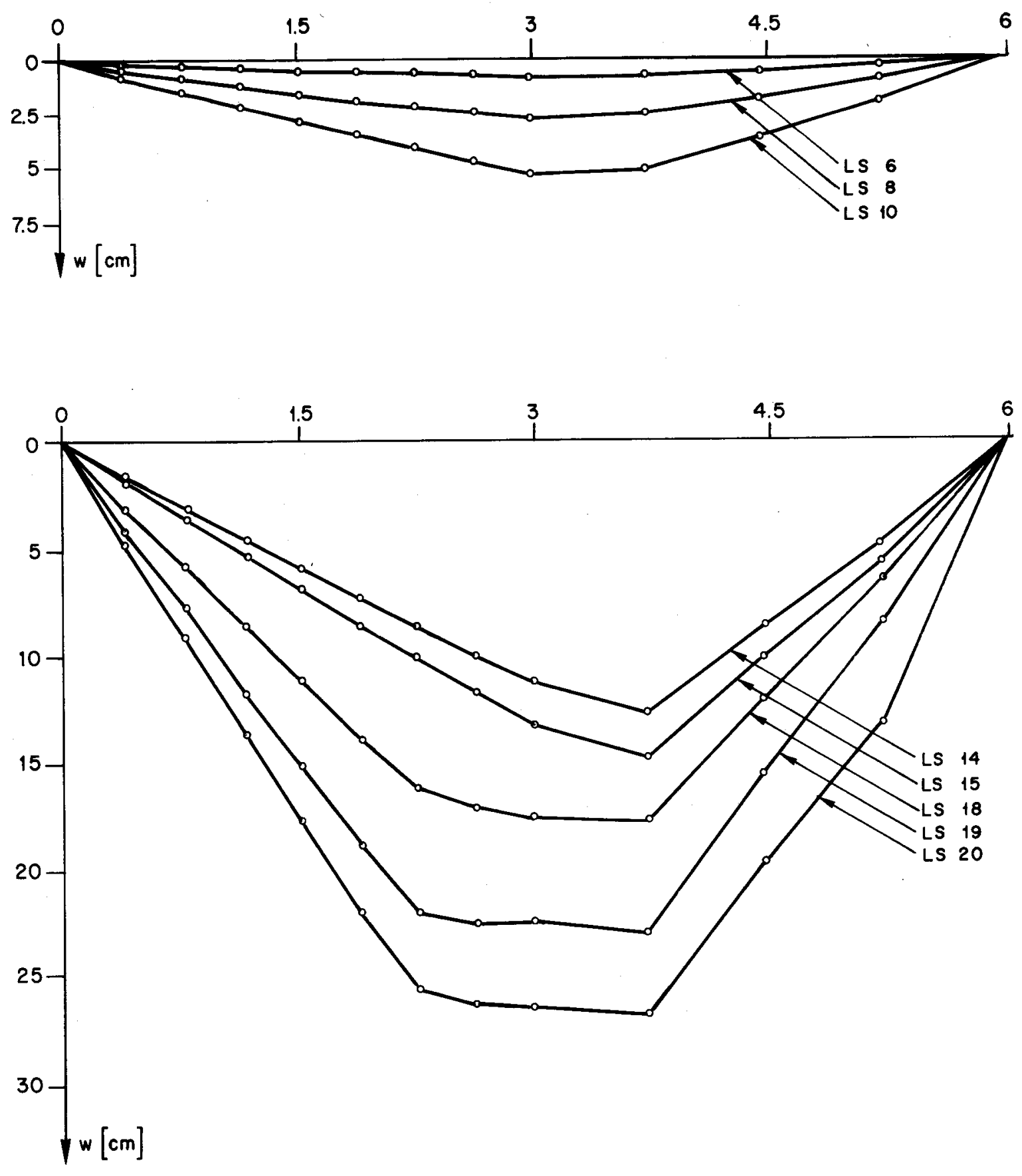

Bild 57: Verlauf der Durchbiegungen Plattenstreifen PS 5 

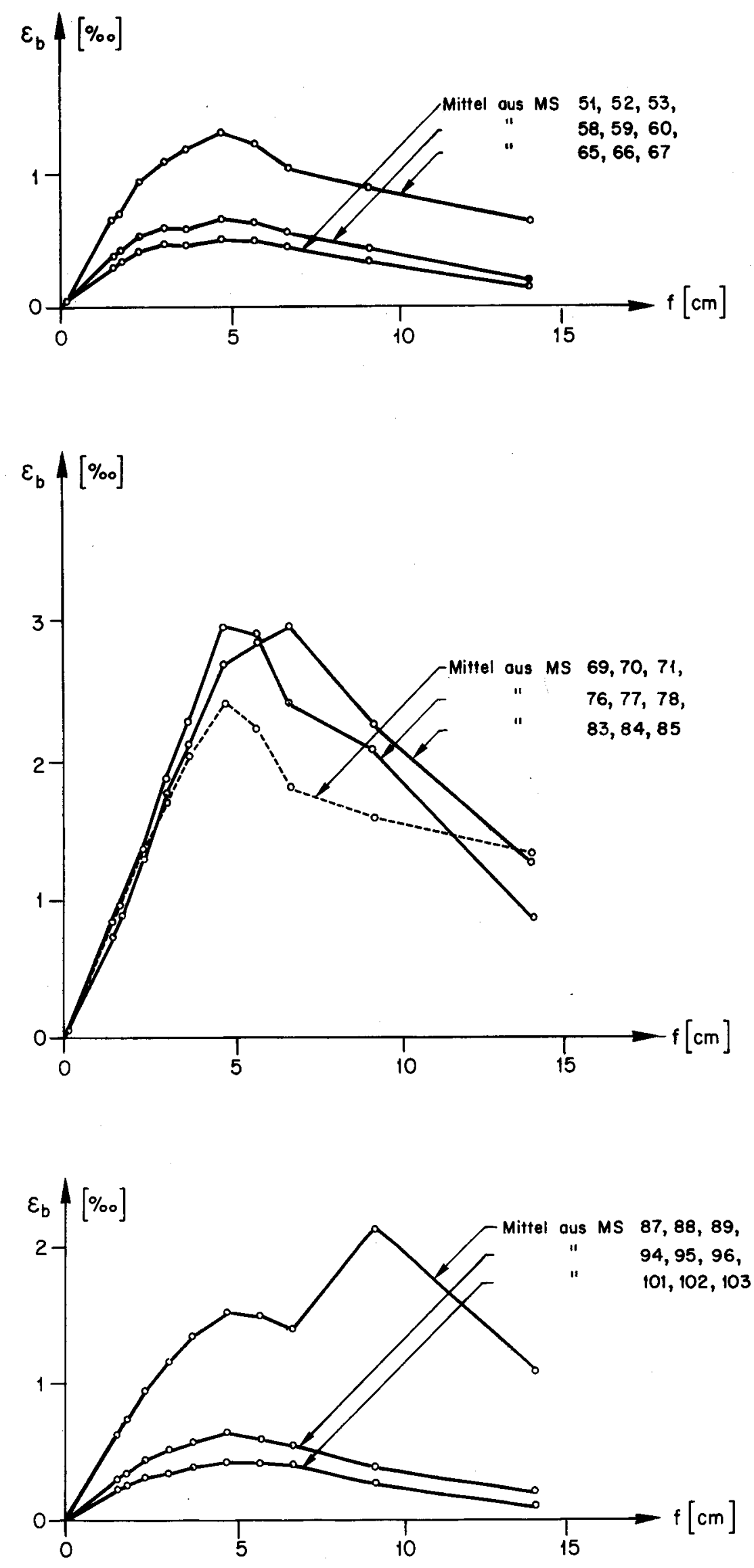

Bild 58 : Betonstauchungen Plattenstreifen PS 1 in Funktion der Mittendurchbiegung 

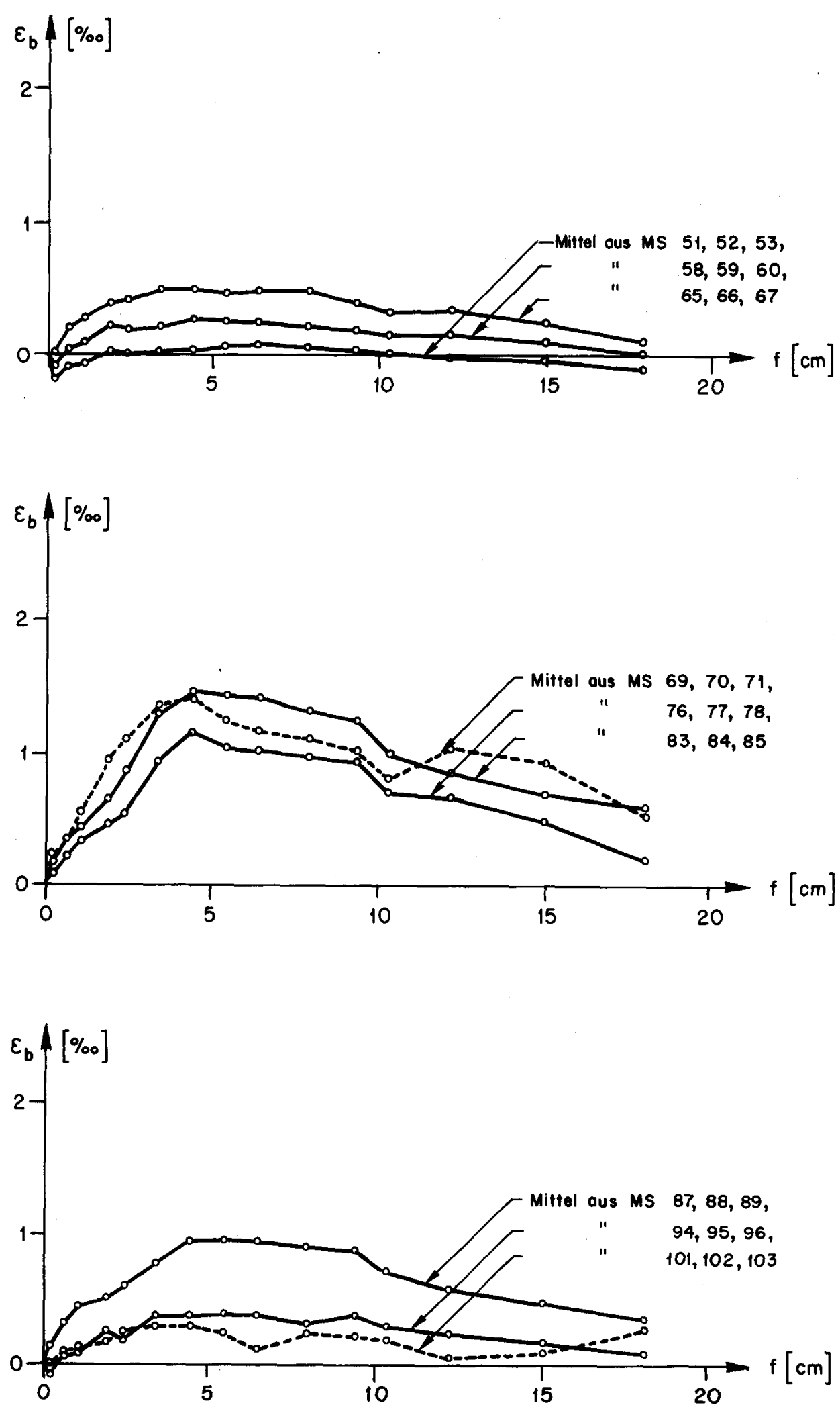

Bild 59 : Betonstauchungen Plattenstreifen PS 2 in Funktion der Mittendurchbiegung 

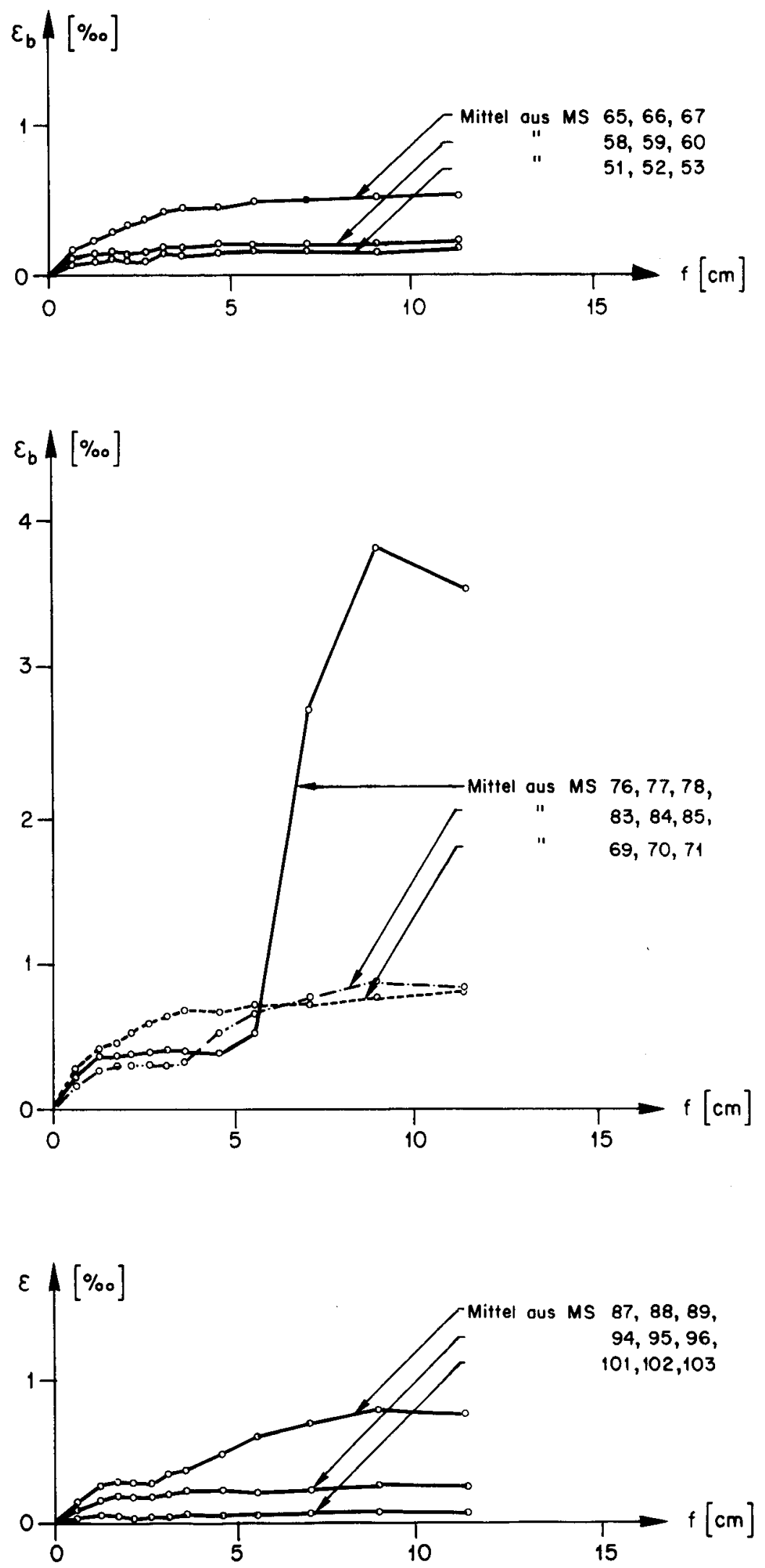

Bild 60 : Betonstauchungen Plattenstreifen PS 3 in Funktion der Mittendurchbiegung 

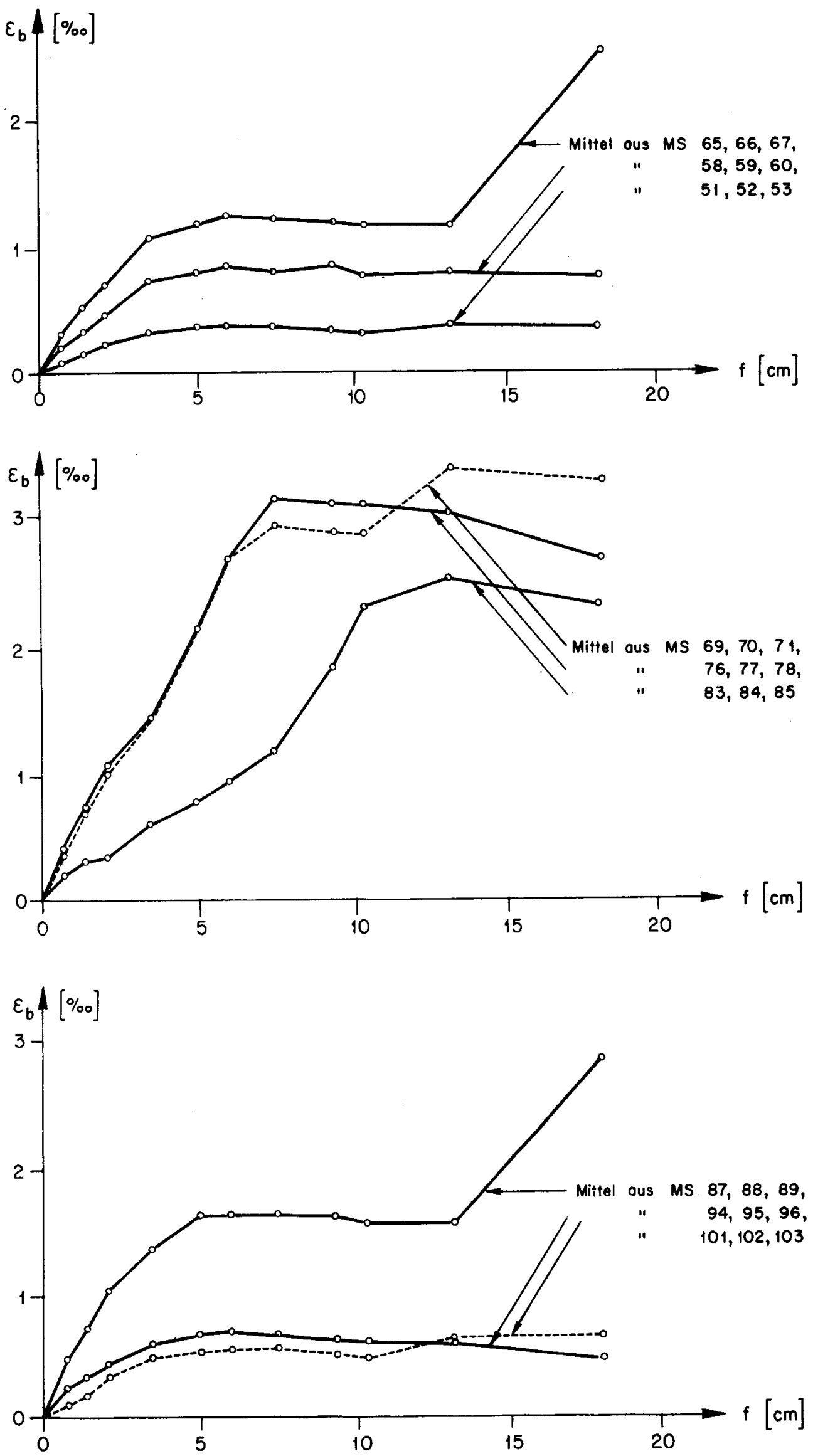

Bild 61 : Betonstauchungen Plattenstreifen PS 4 in Funktion der Mittendurchbiegung 

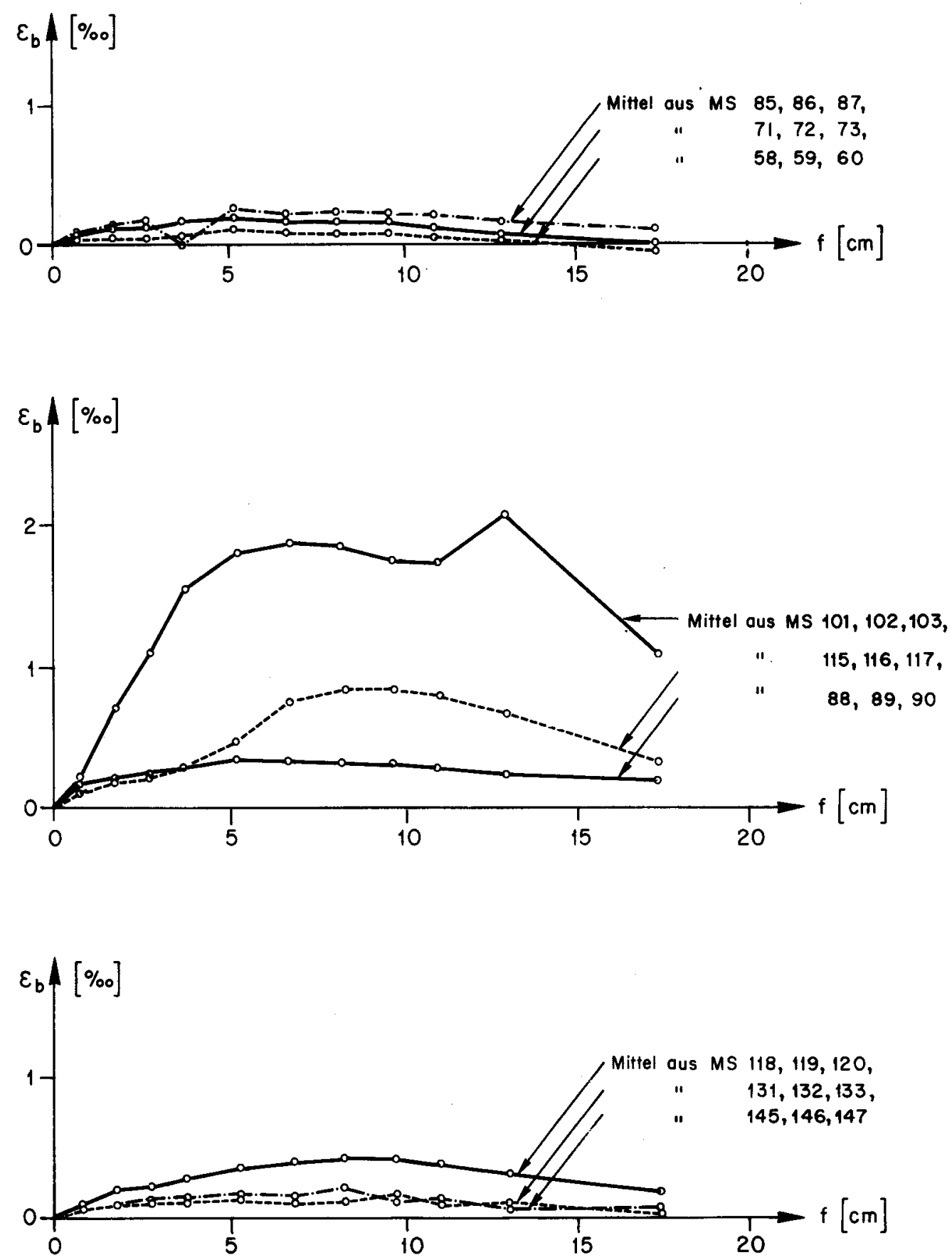

Bild 62 : Betonstauchungen Plattenstreifen PS 5 in Funktion der Mittendurchbiegung 

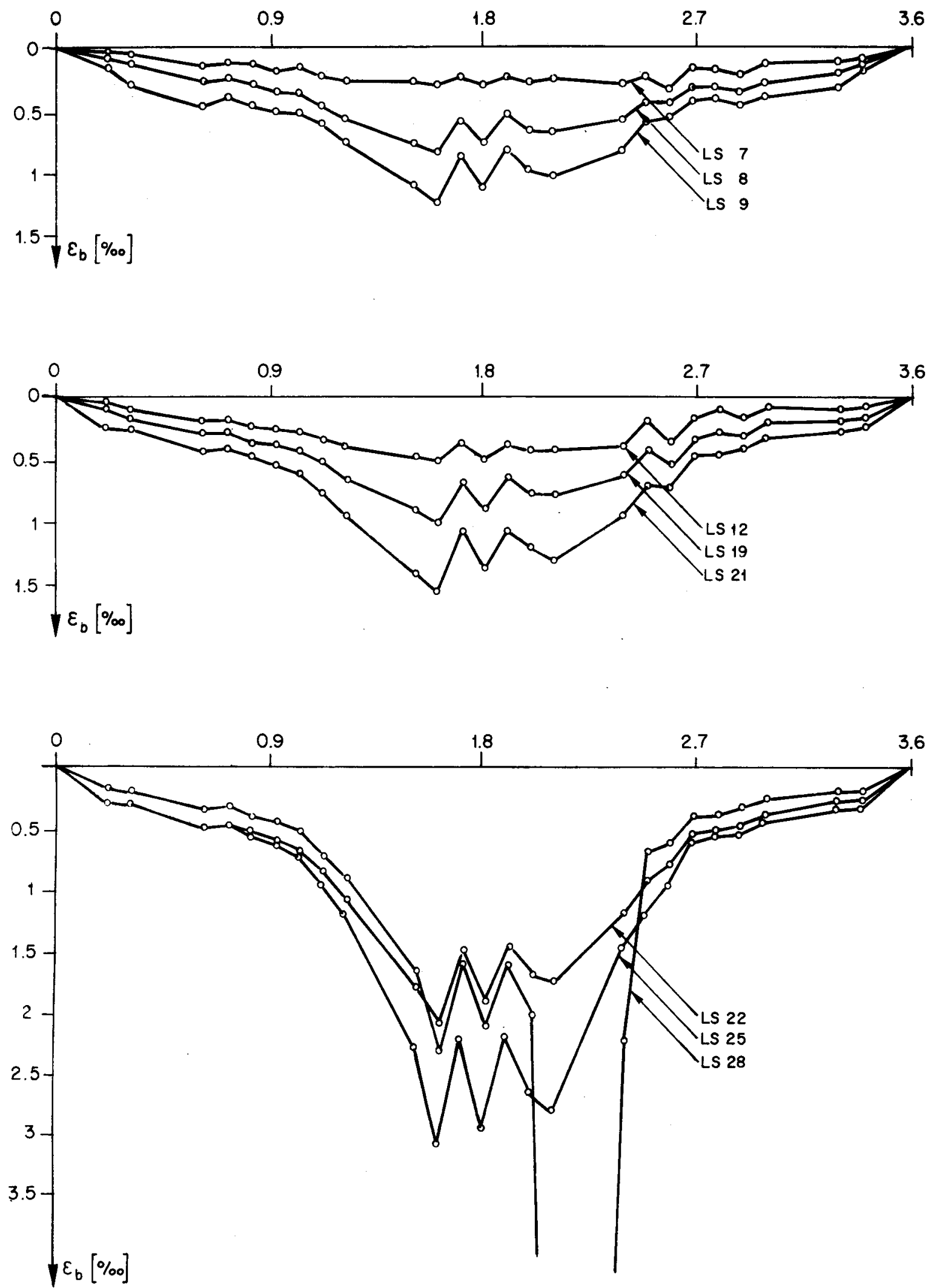

Bild 63: Verlauf der Betonstauchungen Plattenstreifen PS 1 Oberseite (Mittel aus den 2 resp. 3 im Quersinn gemessenen Messstellen) 

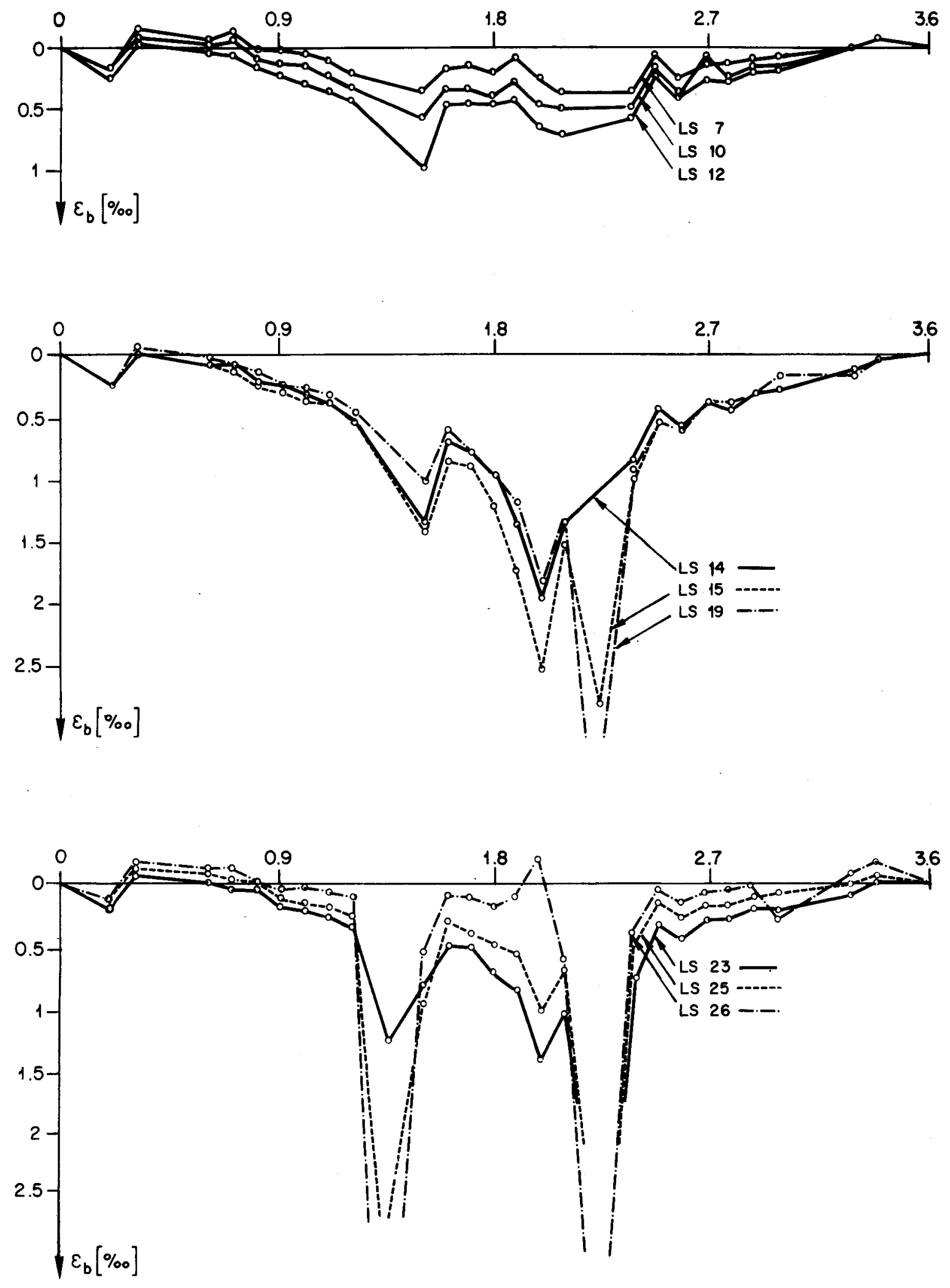

Bild 64: Verlauf der Betonstauchungen Plattenstreifen PS 2 Oberseite (Mittel aus den 2 resp. 3 im Quersinn gemessenen Messstellen) 

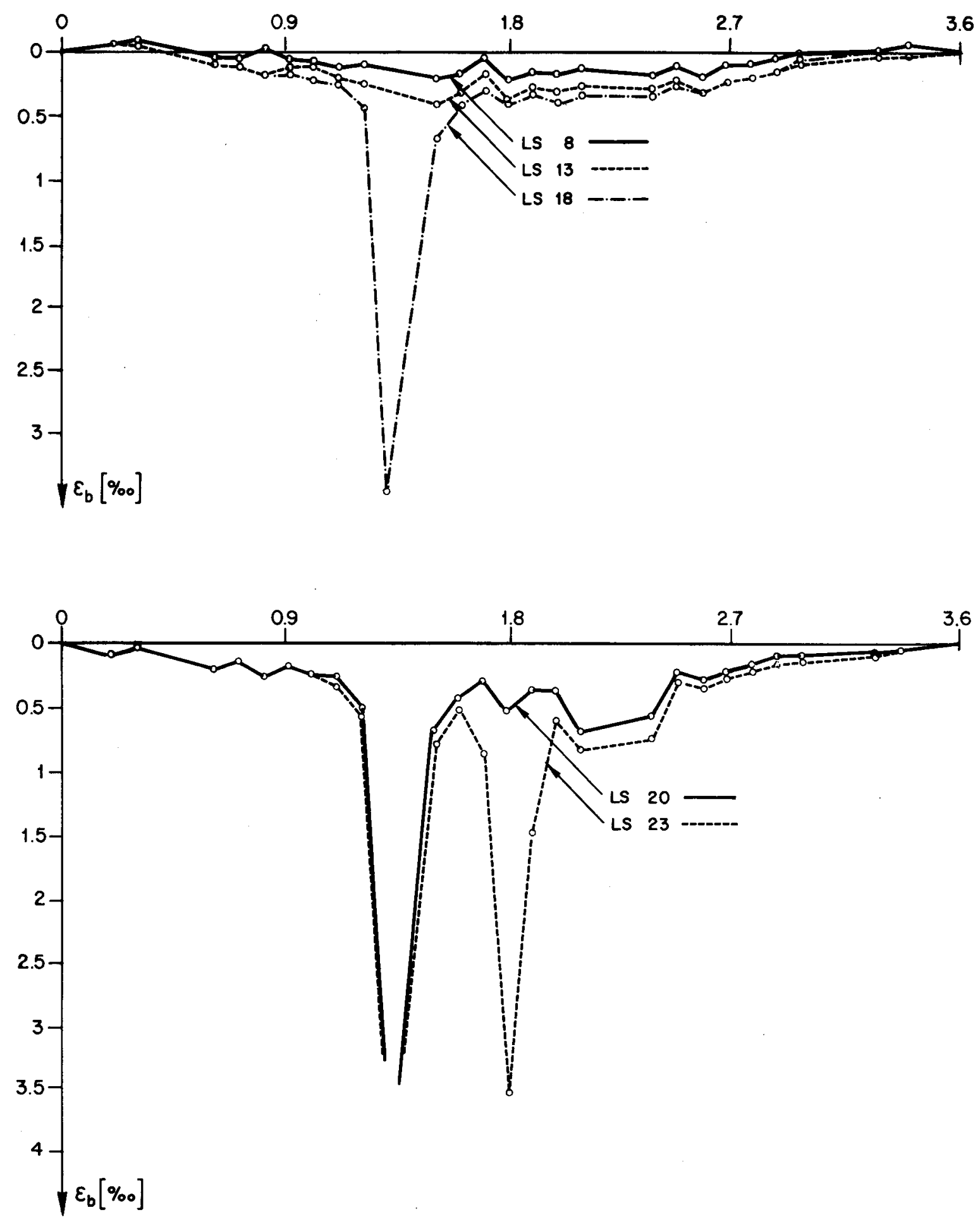

Bild 65 : Verlauf der Betonstauchungen Plattenstreifen PS 3 Oberseite (Mittel aus den 2 resp. 3 im Quersinn gemessenen Messstellen) 

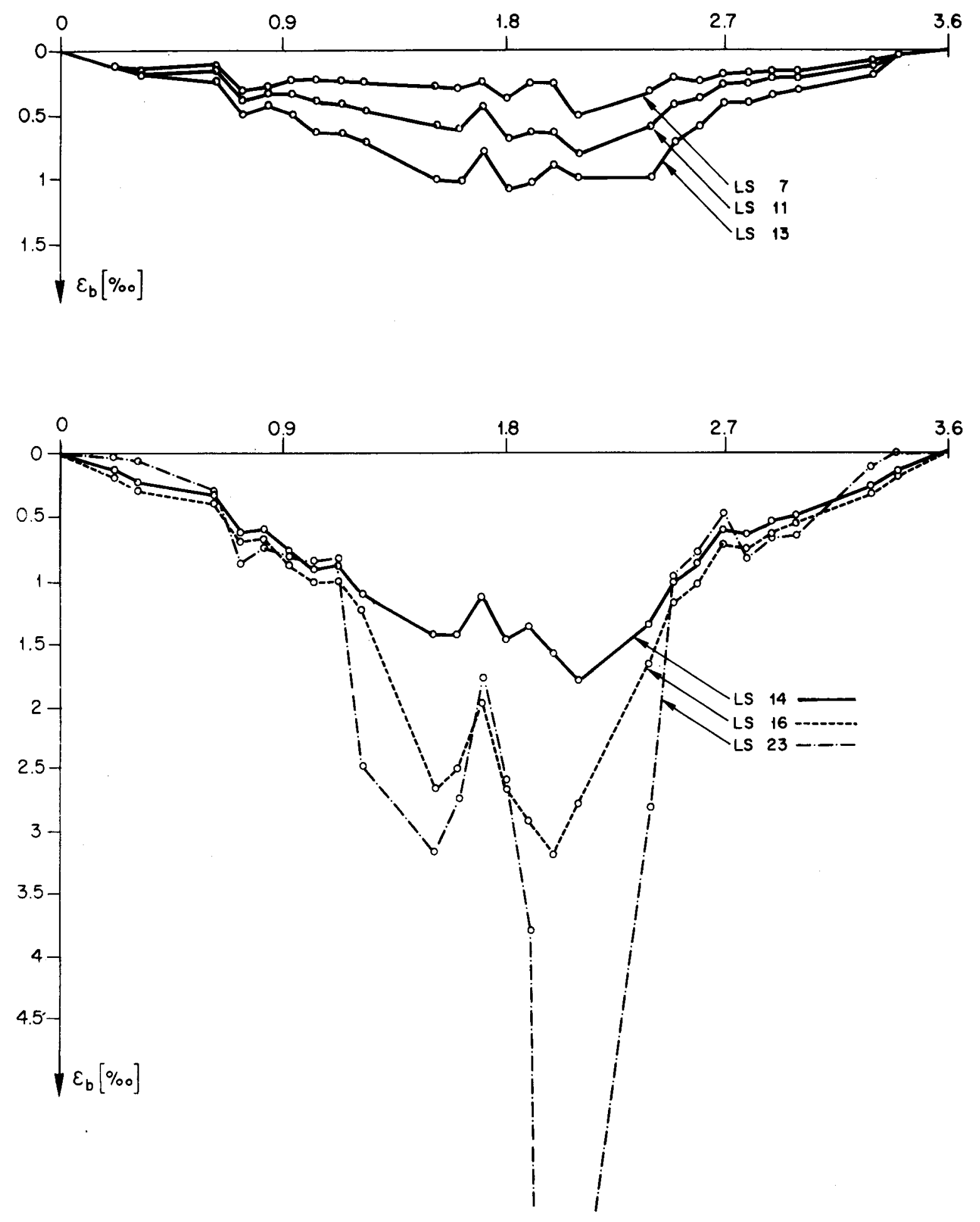

Bild 66: Verlauf der Betonstauchungen Plattenstreifen PS 4 Oberseite (Mittel aus den 2 resp. 3 im Quersinn gemessenen Messstellen) 

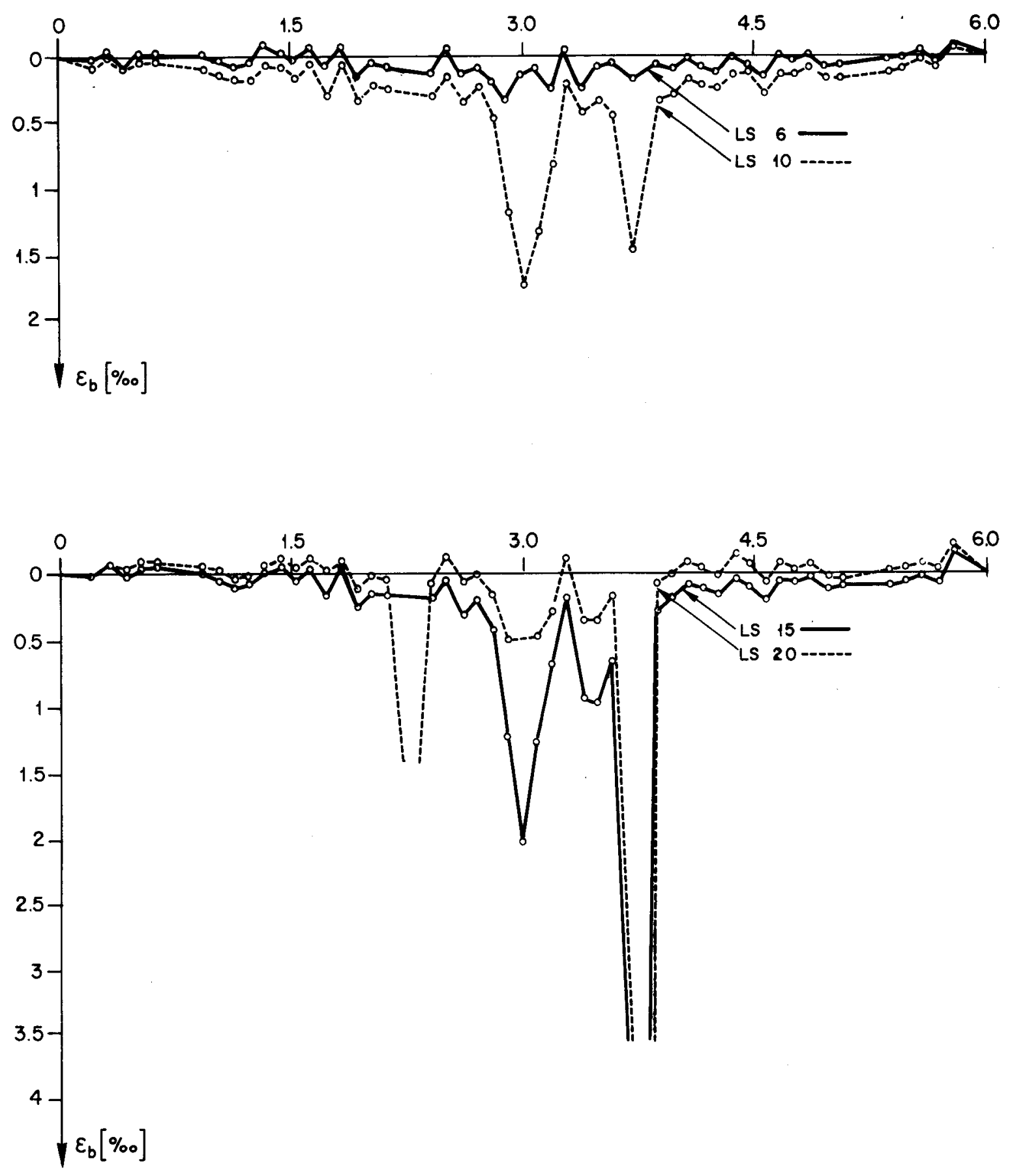

Bild 67: Verlauf der Betonstauchungen Plattenstreifen PS 5 Oberseite (Mittel aus den 2 resp. 3 im Quersinn gemessenen Messstellen) 
Loststufe

LS 4

\begin{tabular}{|l|l|}
\hline 140 & 10 \\
\hline
\end{tabular}

LS 5

45) $31115 \quad(10 \quad 10) \quad 28) 113 \quad 16$

LS 11

5) 5) 51 5) 3),

LS 13

10) 10) 5) 5) $10 / 15$ is

LS 15

20) 15$)$ 5) 5) $15(1 / 5 \quad 15$

LS 19

30) 25) 151 15) 28$)(10 \quad 15$

LS 21

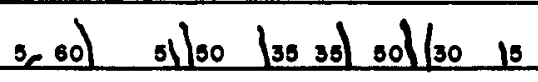

LS 28

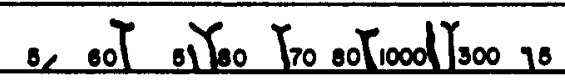

Bild 68: Rissverlauf Plattenstreifen PS 1 (Rissweiten in 1/100 mm) 
Loststufe

LS 3

\begin{tabular}{|l|l|}
\hline $40 \mathrm{Y}$ & 10 \\
\hline
\end{tabular}

LS 5

${ }_{120} 0 / \quad, 5 \quad, \quad 3 \quad 198$

LS 7

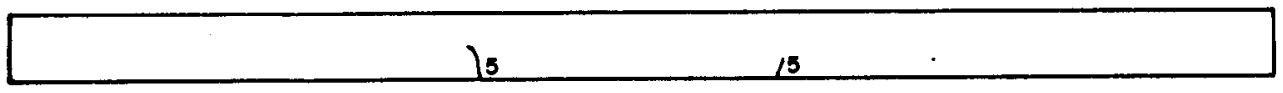

LS 8

770

h

LS 9

18) $\quad 2, \quad 2, \quad 15$

LS 10

$40) \quad 2 \quad 21 \quad 35$

LS 11

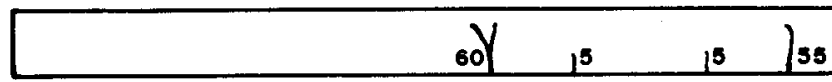

LS 24

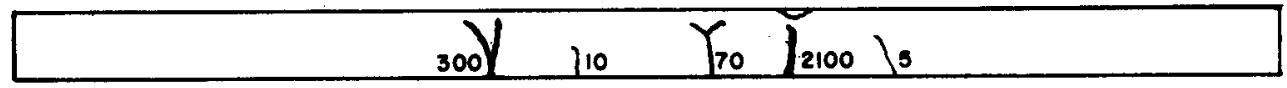

Bild 69 : Rissverlauf Plattenstreifen PS 2 (Rissweiten in 1/100 mm) 
Laststufe

LS 4

10)

LS 5

1201 1

LS 8

8)

LS 9

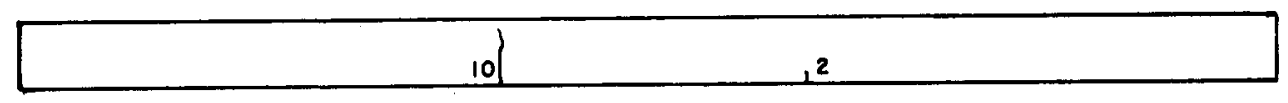

LS 10

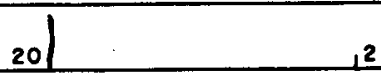

LS 11

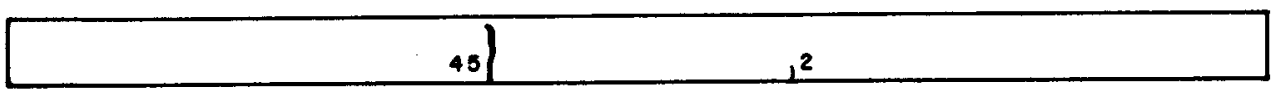

LS 12

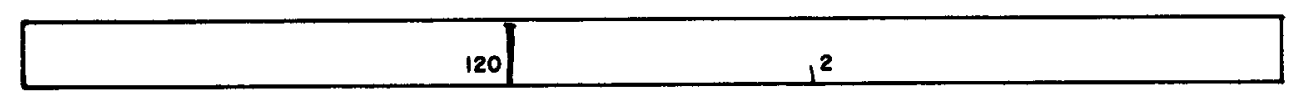

LS 23

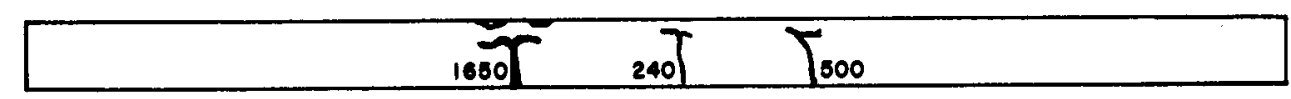

Bild 70: Rissverlauf Plattenstreifen PS 3 (Rissweiten in $1 / 100 \mathrm{~mm}$ ) 
Loststufe

LS 4

31

5)

LS 5

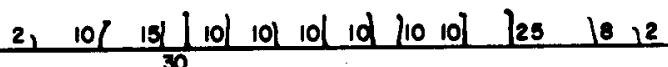

LS 7

$3,5,3,3,3,3,3,3,2,5$

LS 9

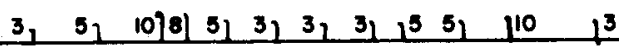

LS 11

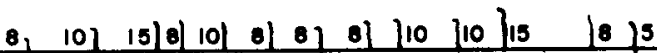

LS 13

8) $15 /\left.\left.30 \int_{10}\right|_{20} l_{20} l_{20} l_{20} \alpha_{20} l_{20} l_{35}\right|_{10} 18$

LS 15

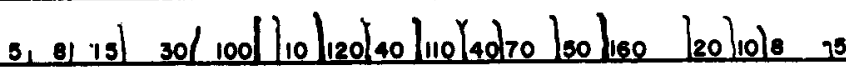

LS 21

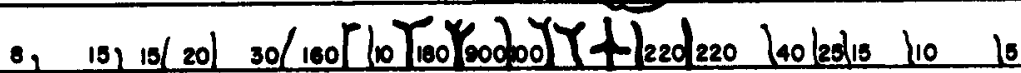

300100180

Bild 71 : Rissverlauf Plattenstreifen PS 4 (Rissweiten in 1/100 mm) 
Loststufe

LS 4

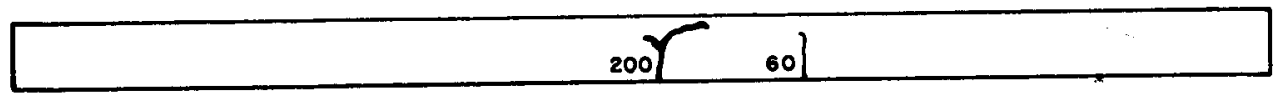

LS 5

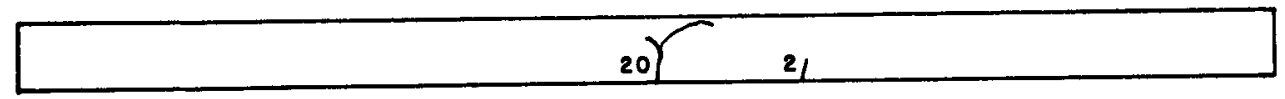

LS 6

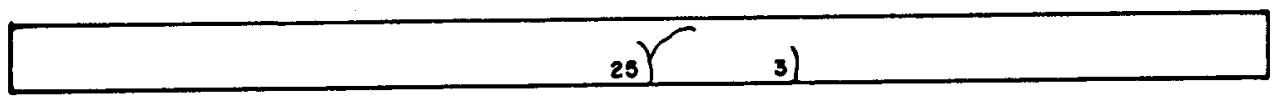

LS 7

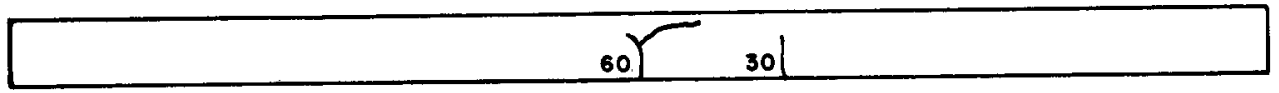

LS 8

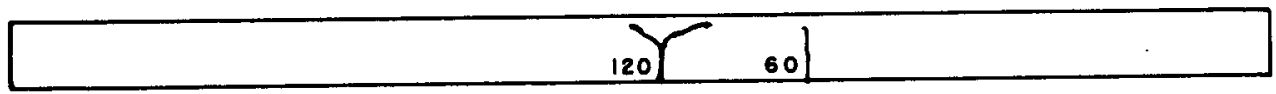

LS 9

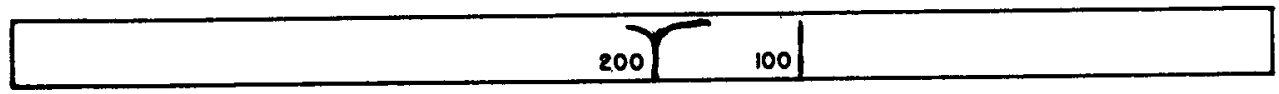

LS 10

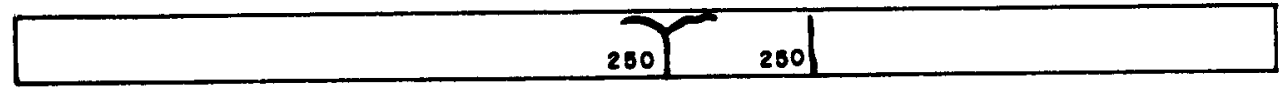

LS 20

\begin{tabular}{|l|l|l|l|l|l|}
\hline 2200 & 500 & or & & 1700 & 1300 \\
\hline
\end{tabular}

Bild 72 : Rissverlauf Plattenstreifen PS 5 (Rissweiten in $1 / 100 \mathrm{~mm}$ ) 


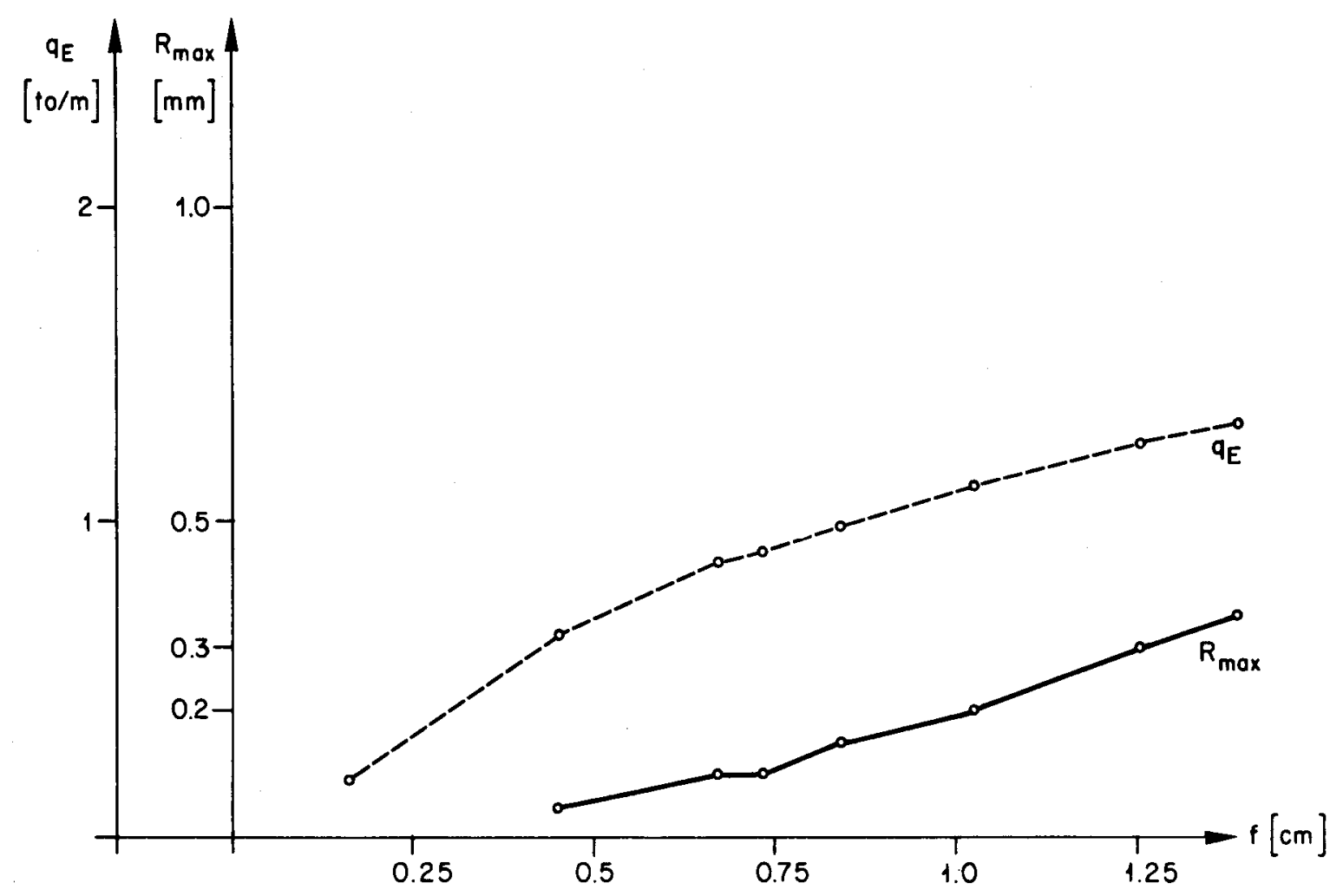

Bild 73: Maximale Rissweite und Belastung Plattenstreifen PS 1 in Funktion der Mittendurchbiegung

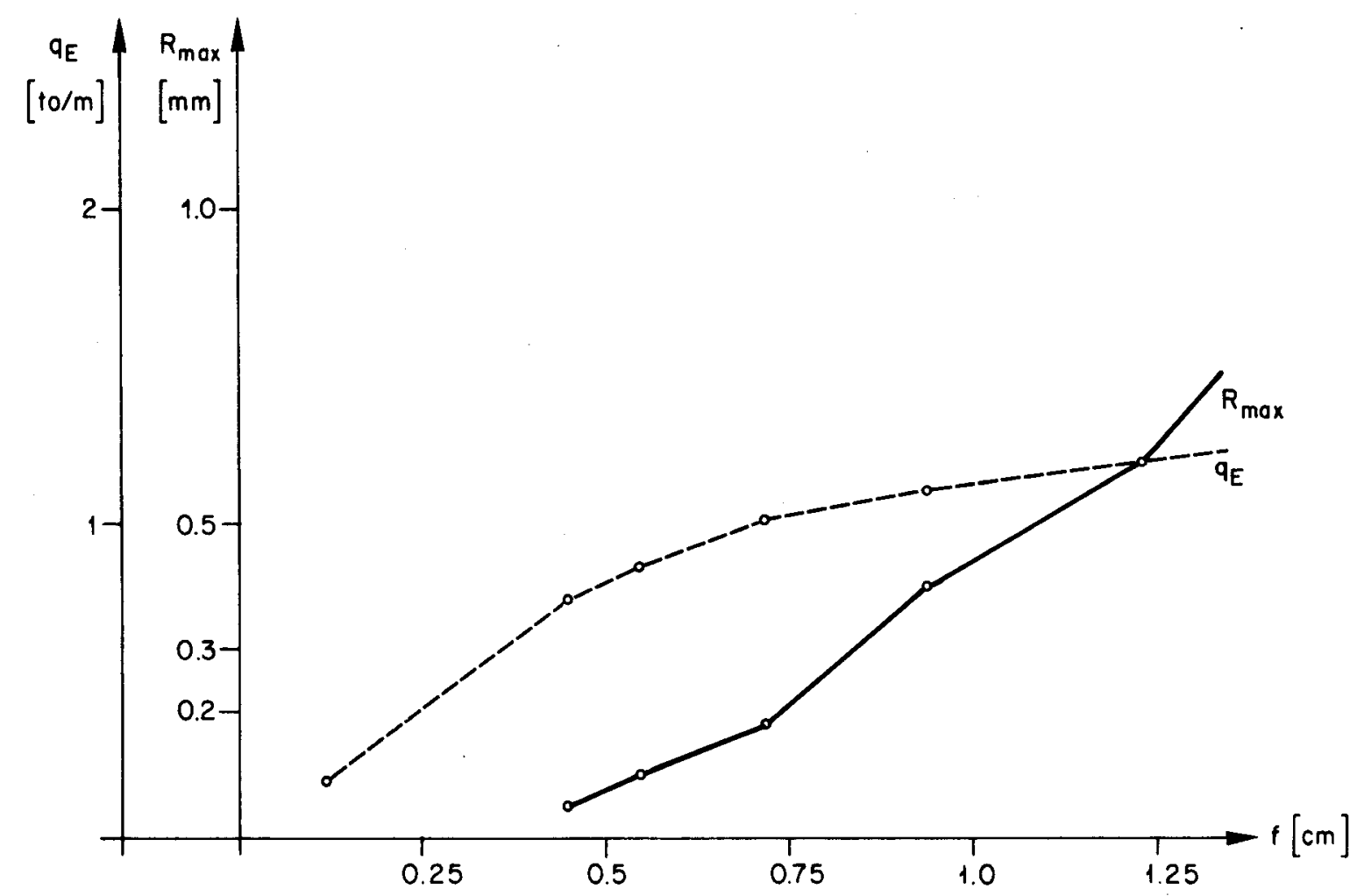

Bild 74: Maximale Rissweite und Belastung Plattenstreifen PS 2 in Funktion der Mittendurchbiegung 


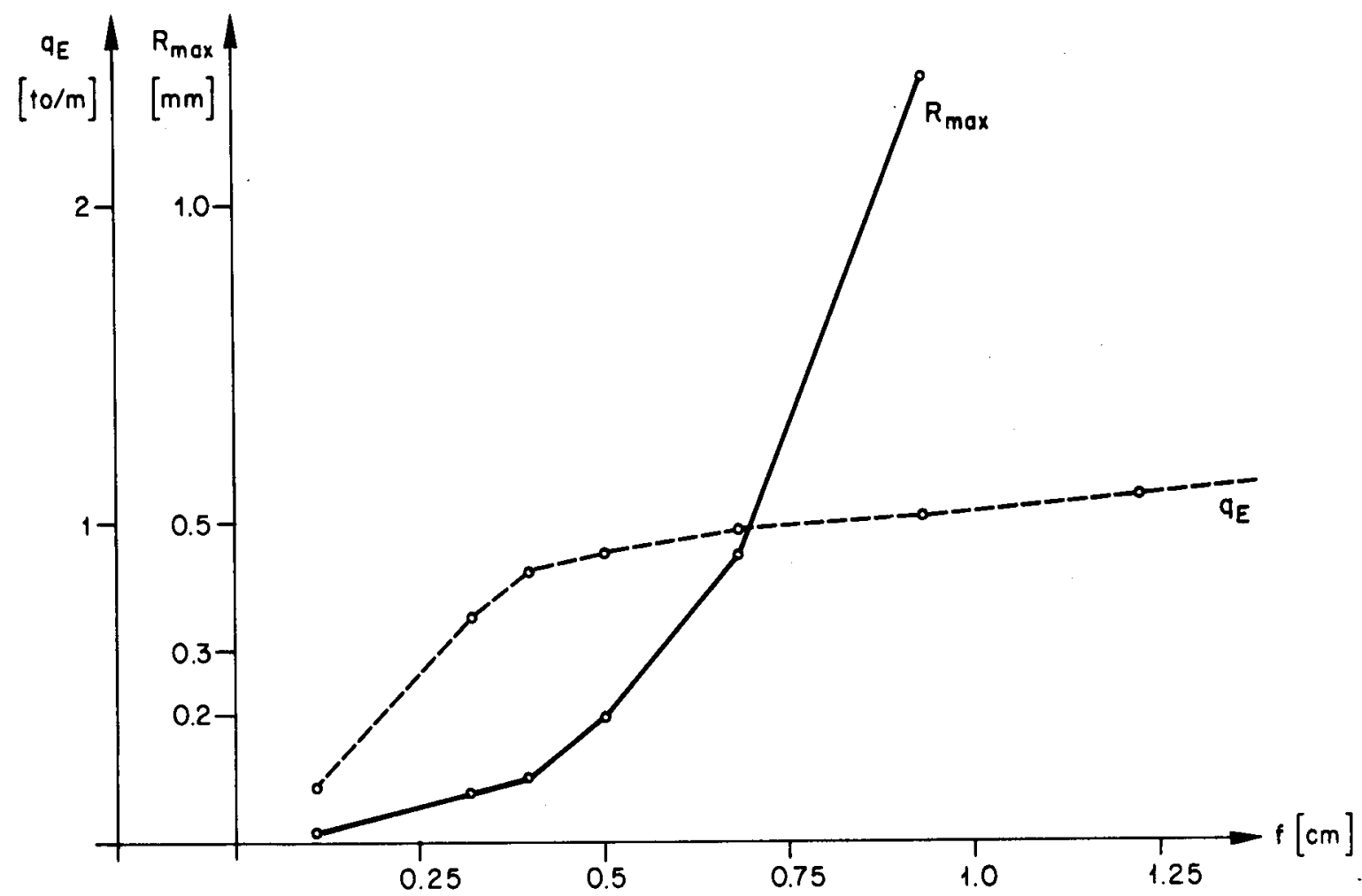

Bild 75: Maximale Rissweite und Belastung Plattenstreifen PS 3 in Funktion der Mittendurchbiegung

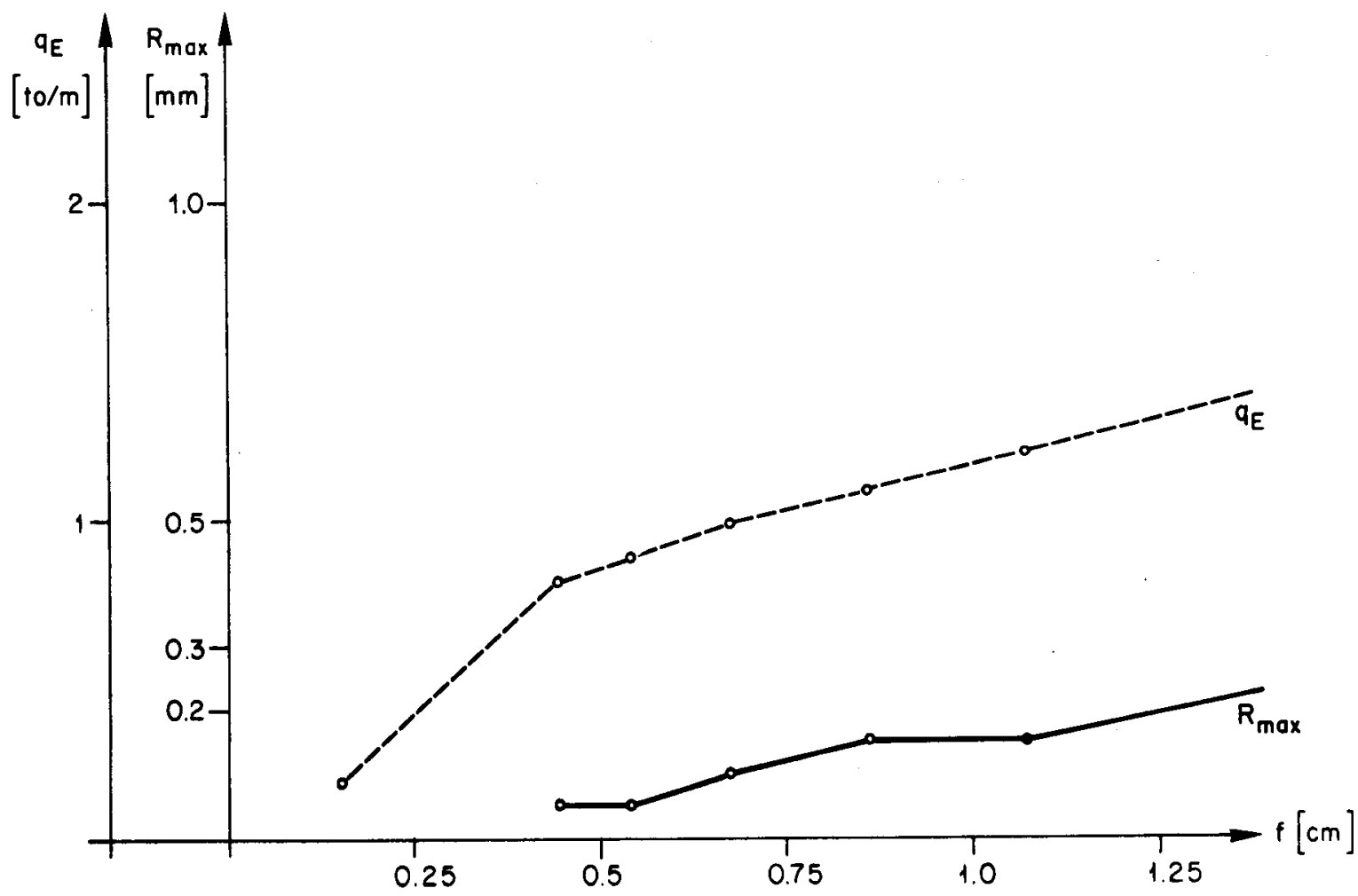

Bild 76: Maximale Rissweite und Belastung Plattenstreifen PS 4 in Funktion der Mittendurchbiegung 


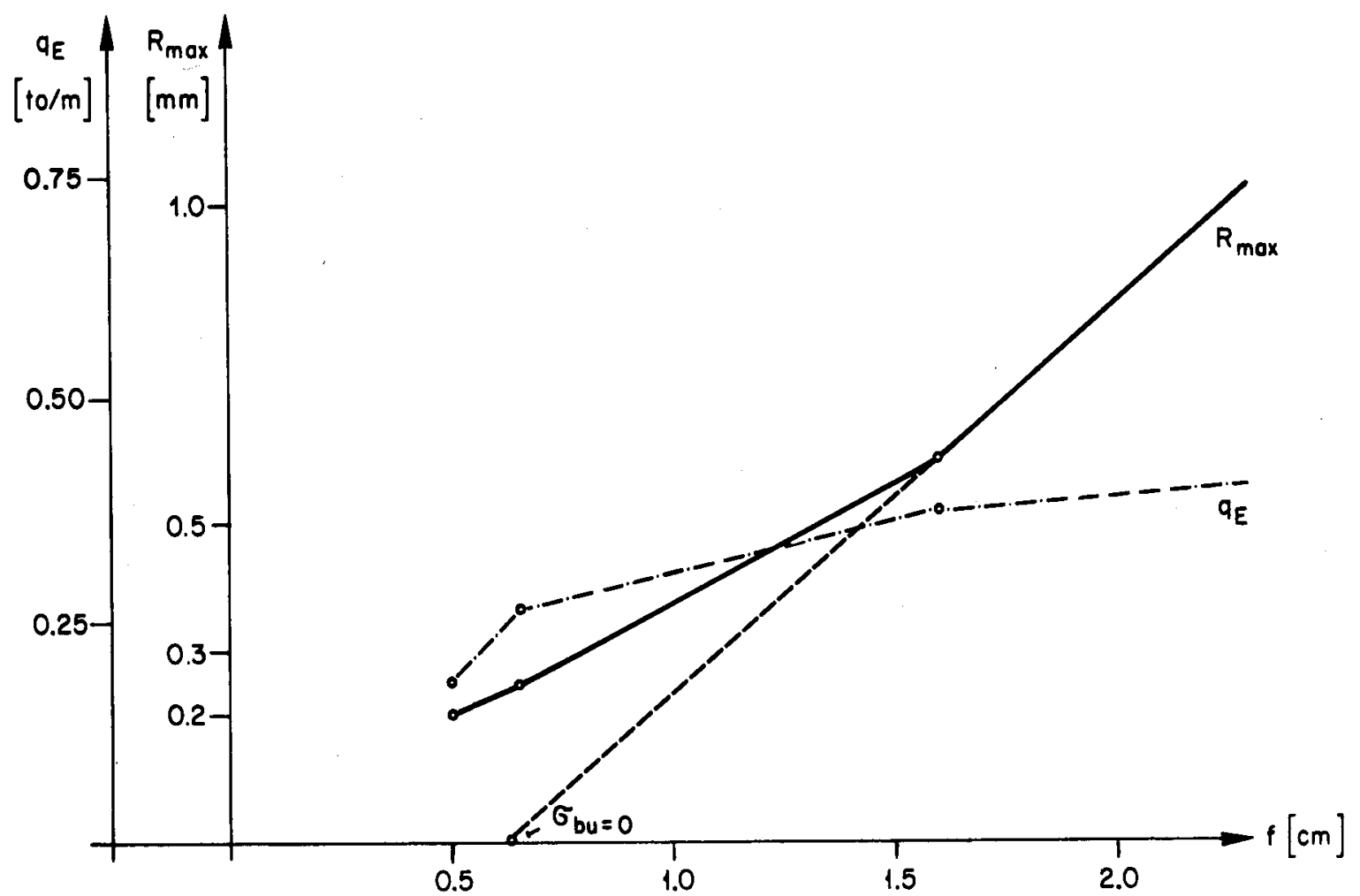

Bild 77: Maximale Rissweite und Belastung Plattenstreifen PS 5 in Funktion der Mittendurchbiegung

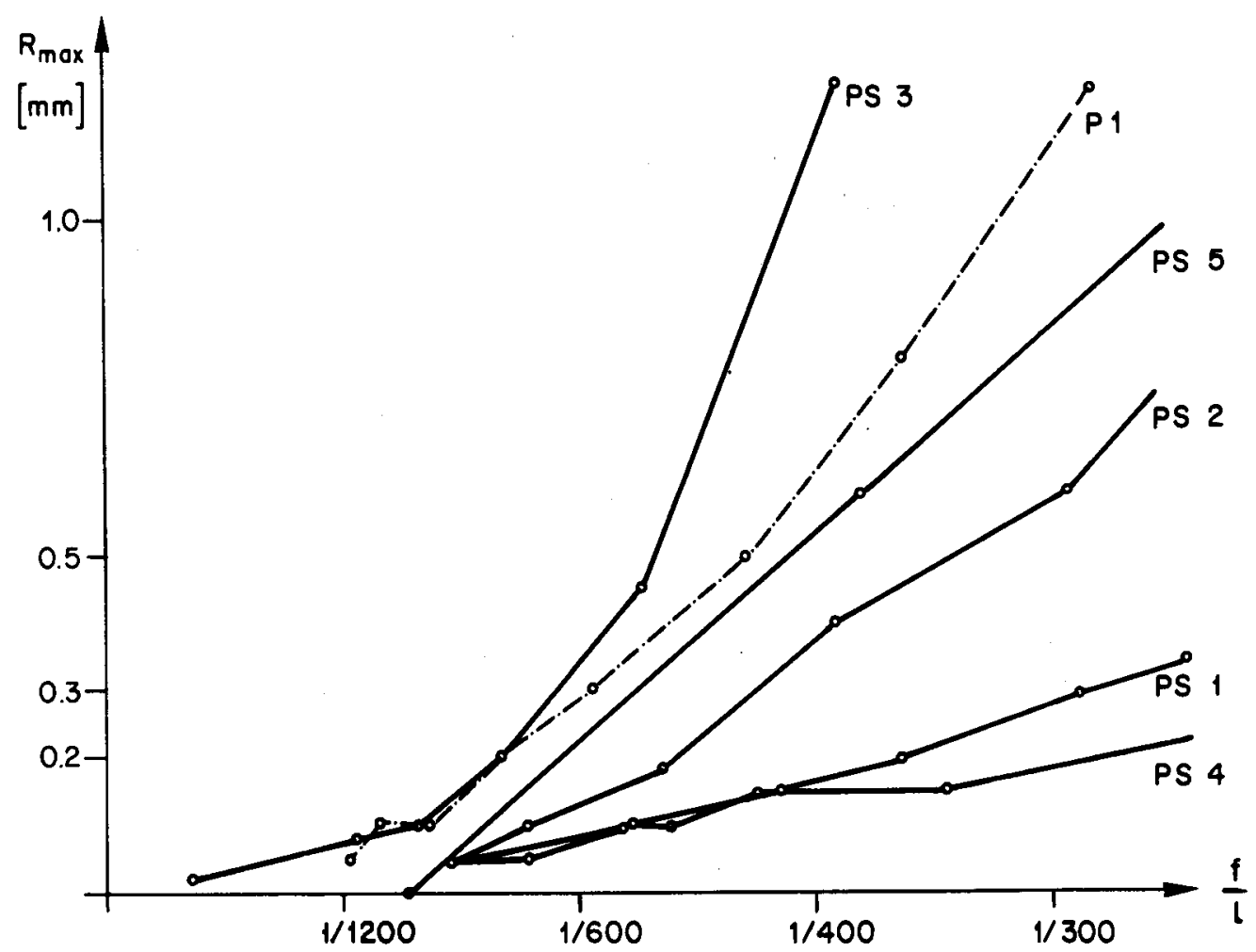

Bild 78: Maximale Rissweiten Platte P 1 und Plattenstreifen PS 1 bis PS 5 in Funktion der Mittendurchbiegung 


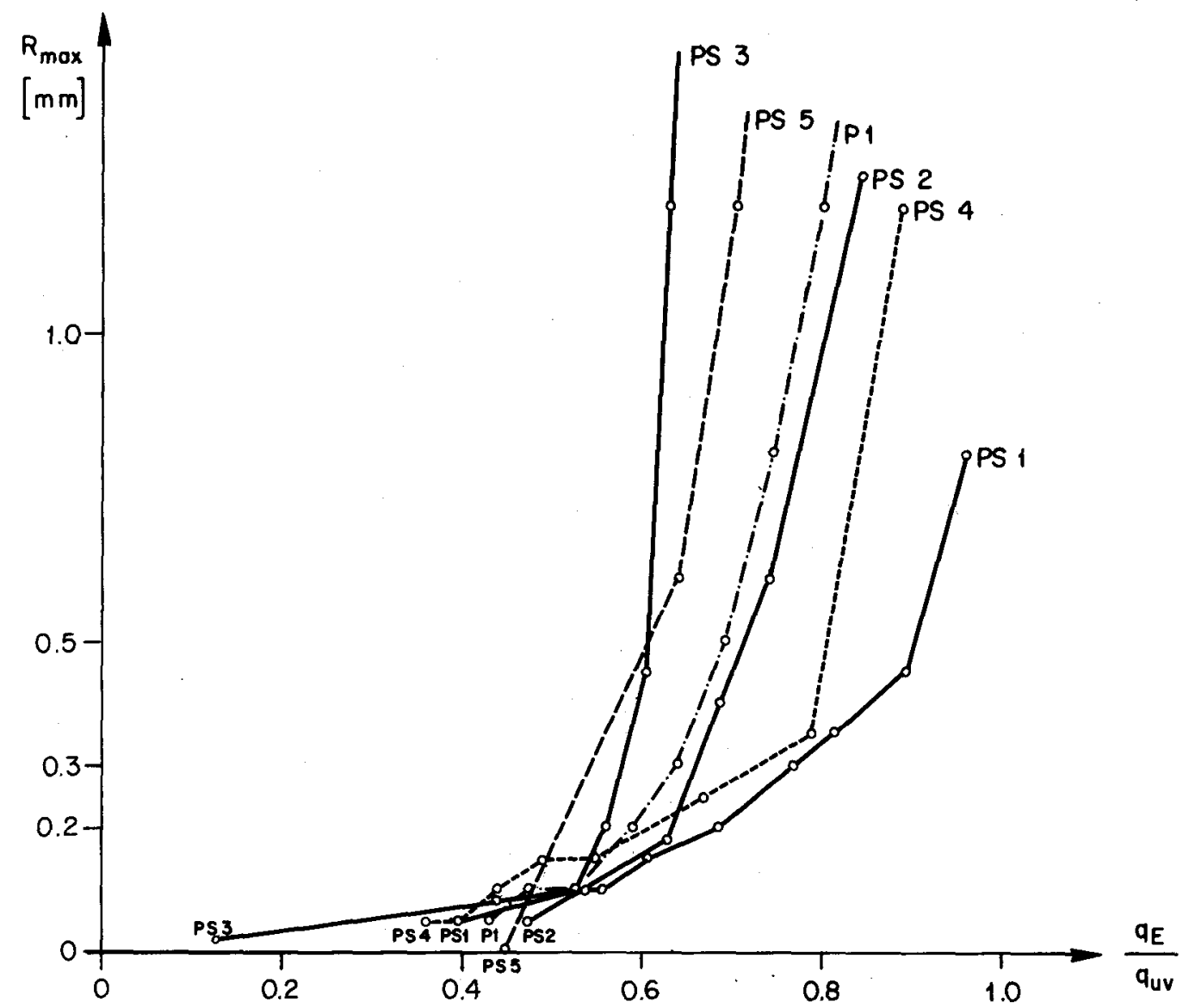

Bild 79: Maximale Rissweiten Platte P 1 und Plattenstreifen PS 1 bis PS 5 in Funktion der Belastung 


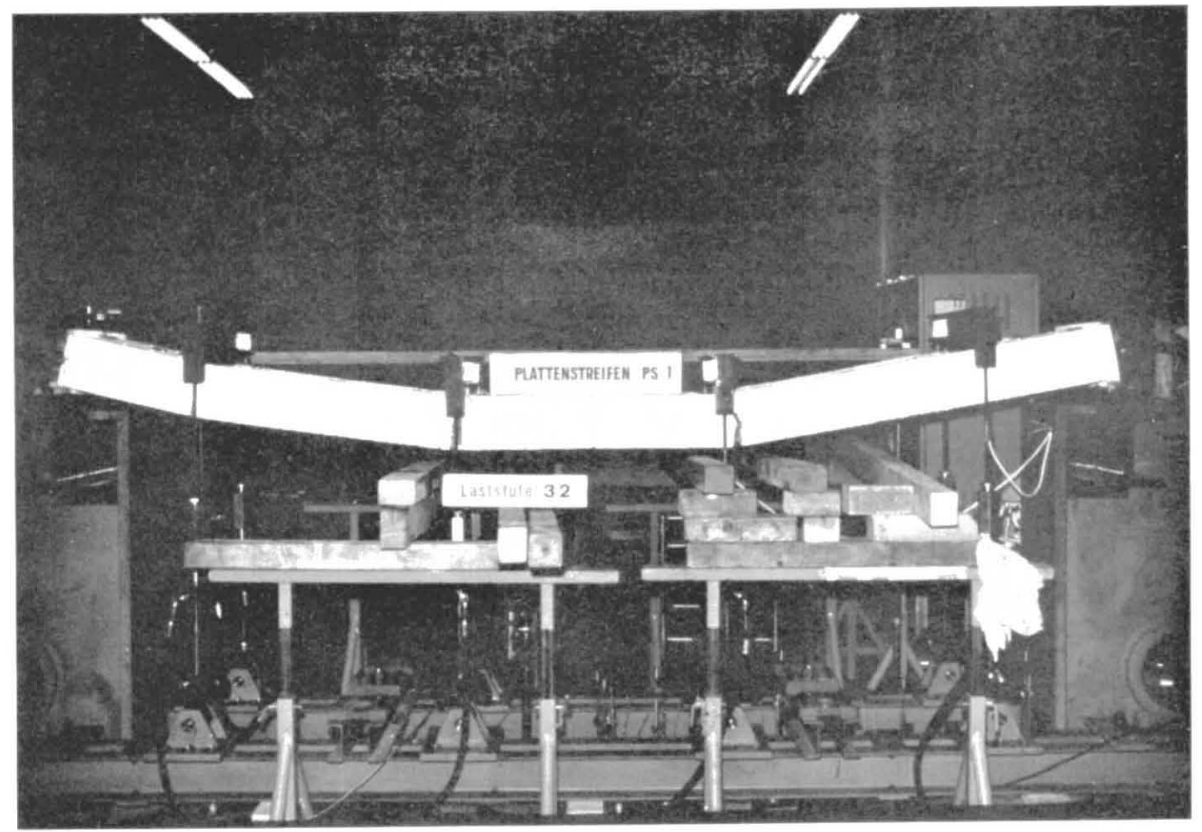

Bild 80: Rissverteilung und Verformung Plattenstreifen PS 1

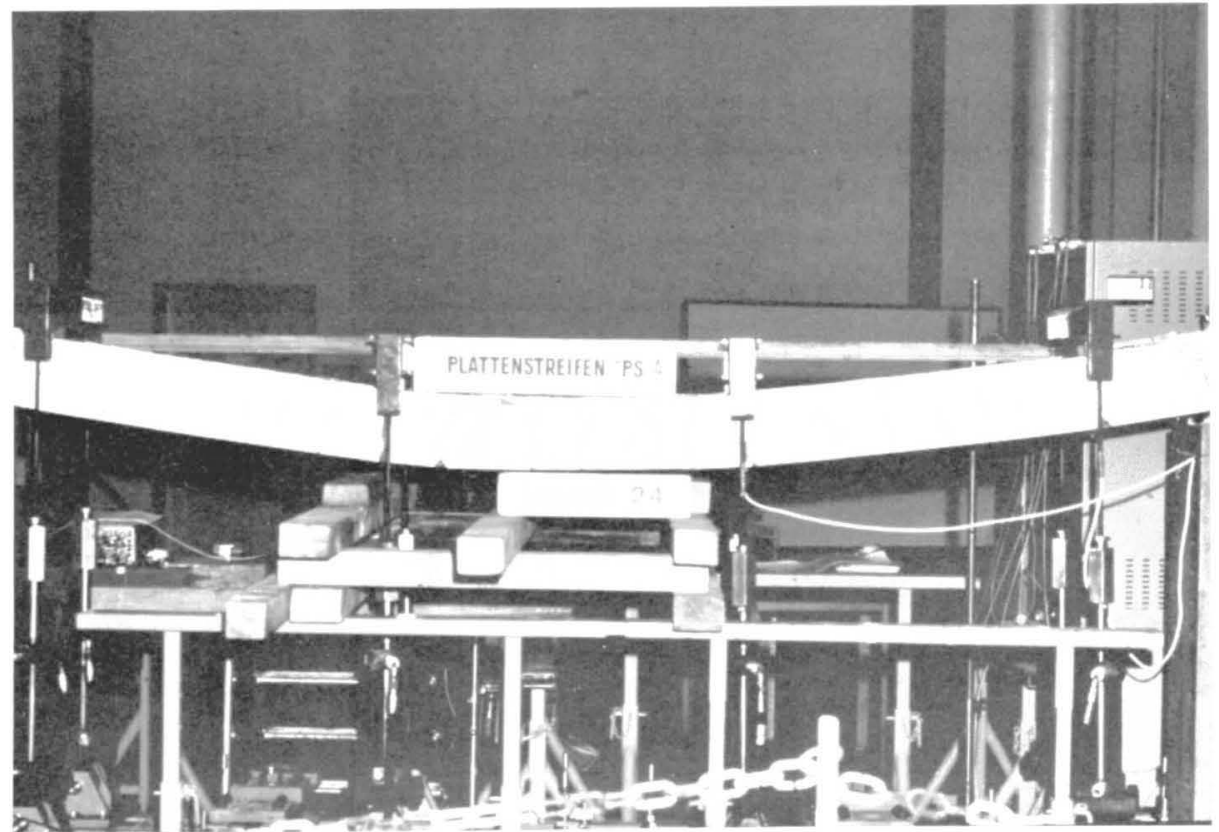

Bild 81: Rissverteilung und Verformung Plattenstreifen PS 4 


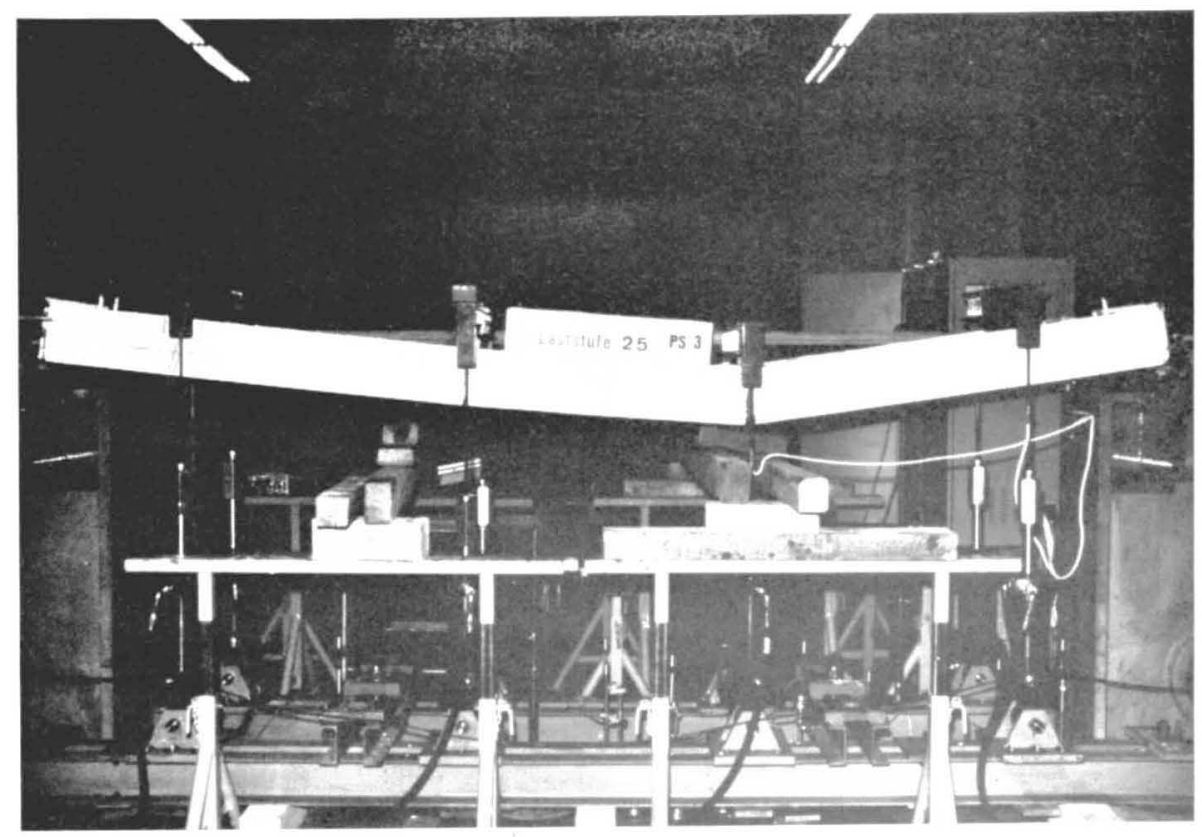

Bild 82: Rissverteilung und Verformung Plattenstreifen PS 3

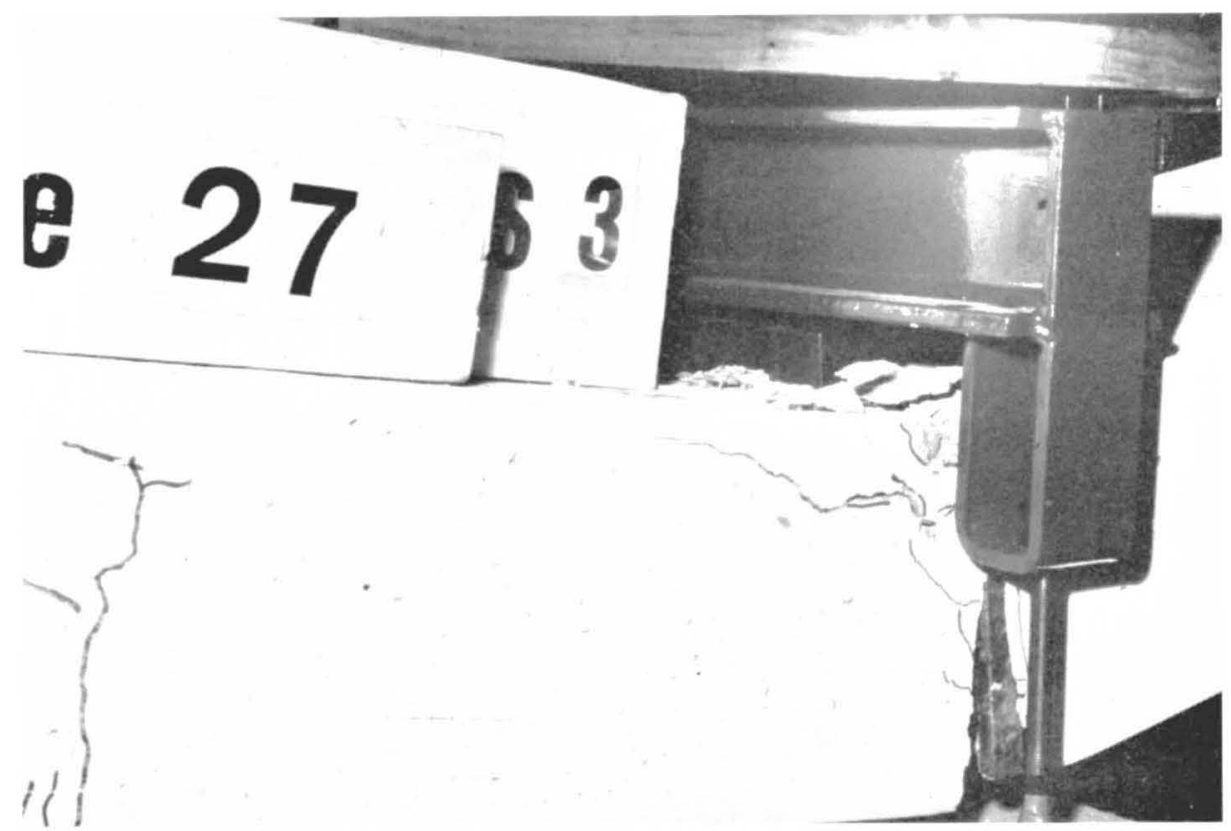

Bild 83: Detail Gelenk Plattenstreifen PS 3 


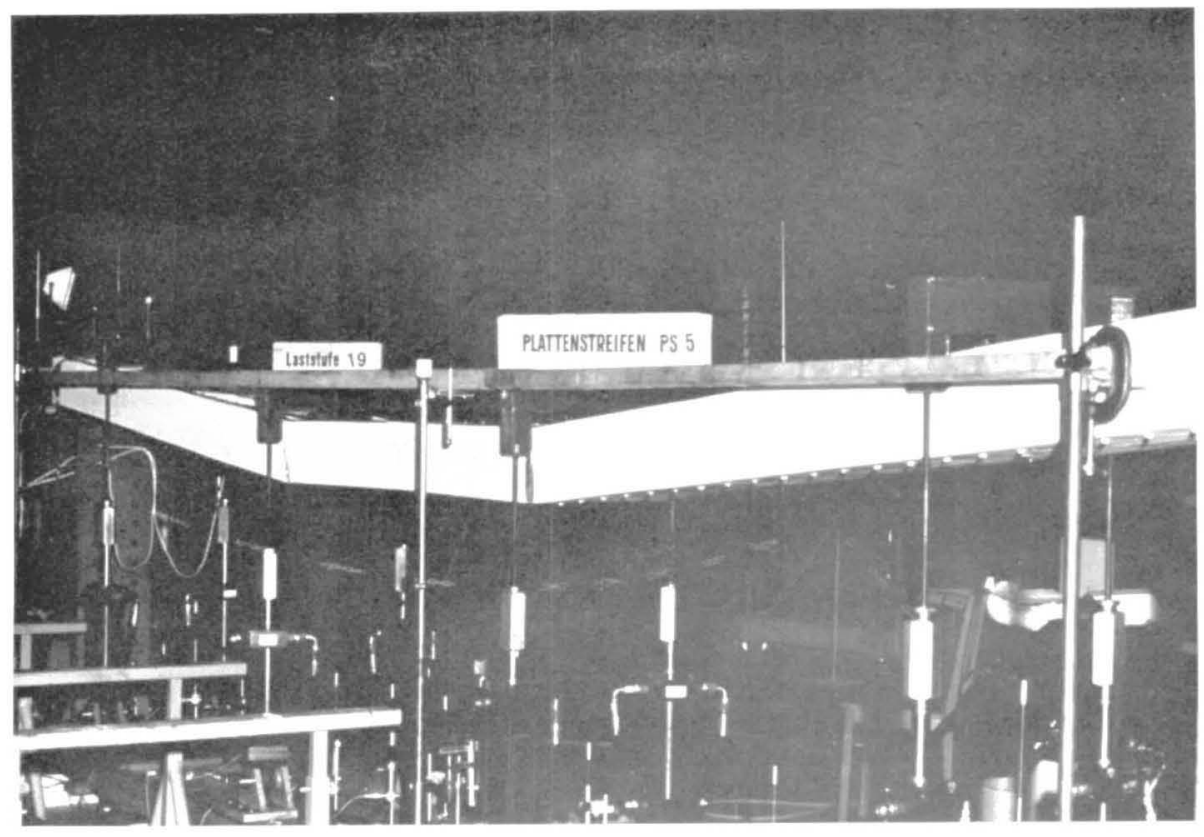

Bild 84: Rissverteilung und Verformung Plattenstreifen PS 5

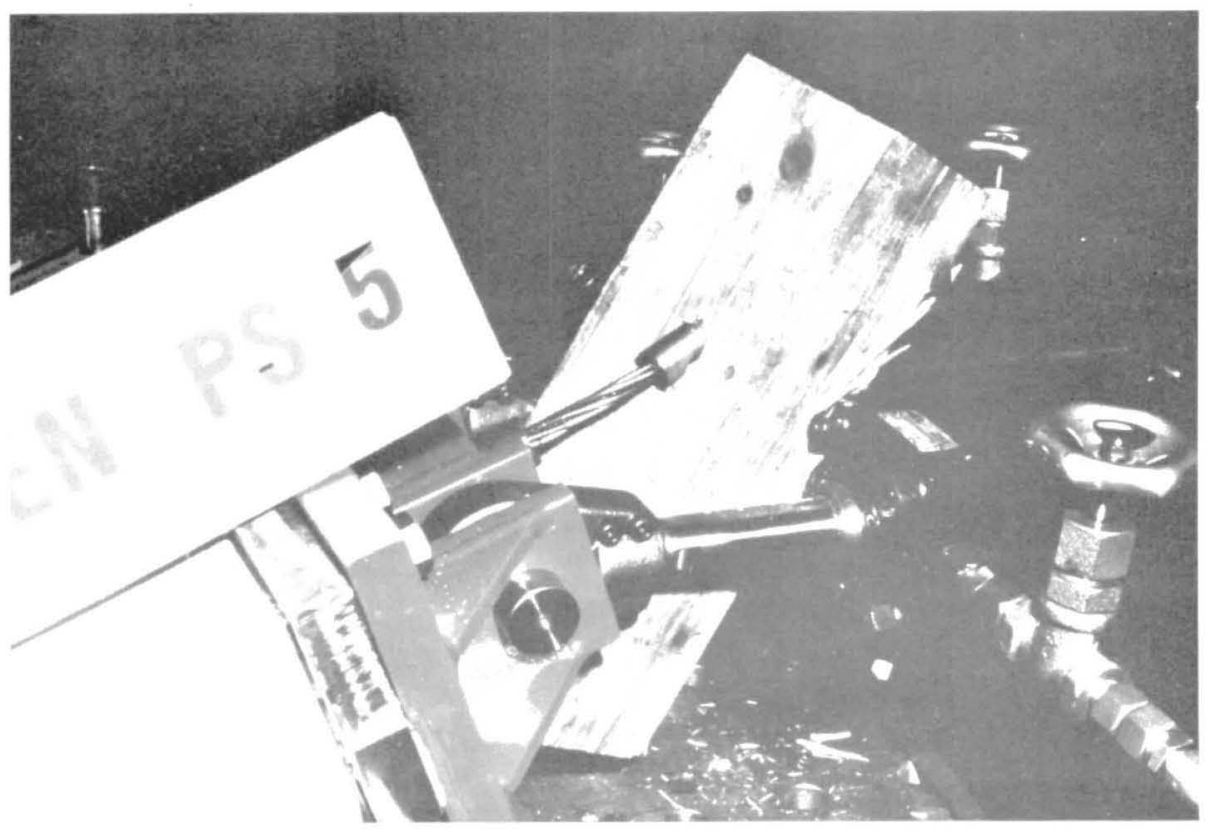

Bild 85: Endverankerung nach Bruch (Reissen der Litze) 
Im folgenden soll kurz ein theoretisches Modell skizziert werden, welches das Tragverhalten von vorgespannten Platten ohne Verbund beschreibt. Eine ausführliche Darstellung dieses Modelles ist für einen folgenden Bericht vorgesehen. In Bild 86 ist anhand einer Einfeldplatte gezeigt, wie man sich die globale Tragwirkung vorstellen kann.

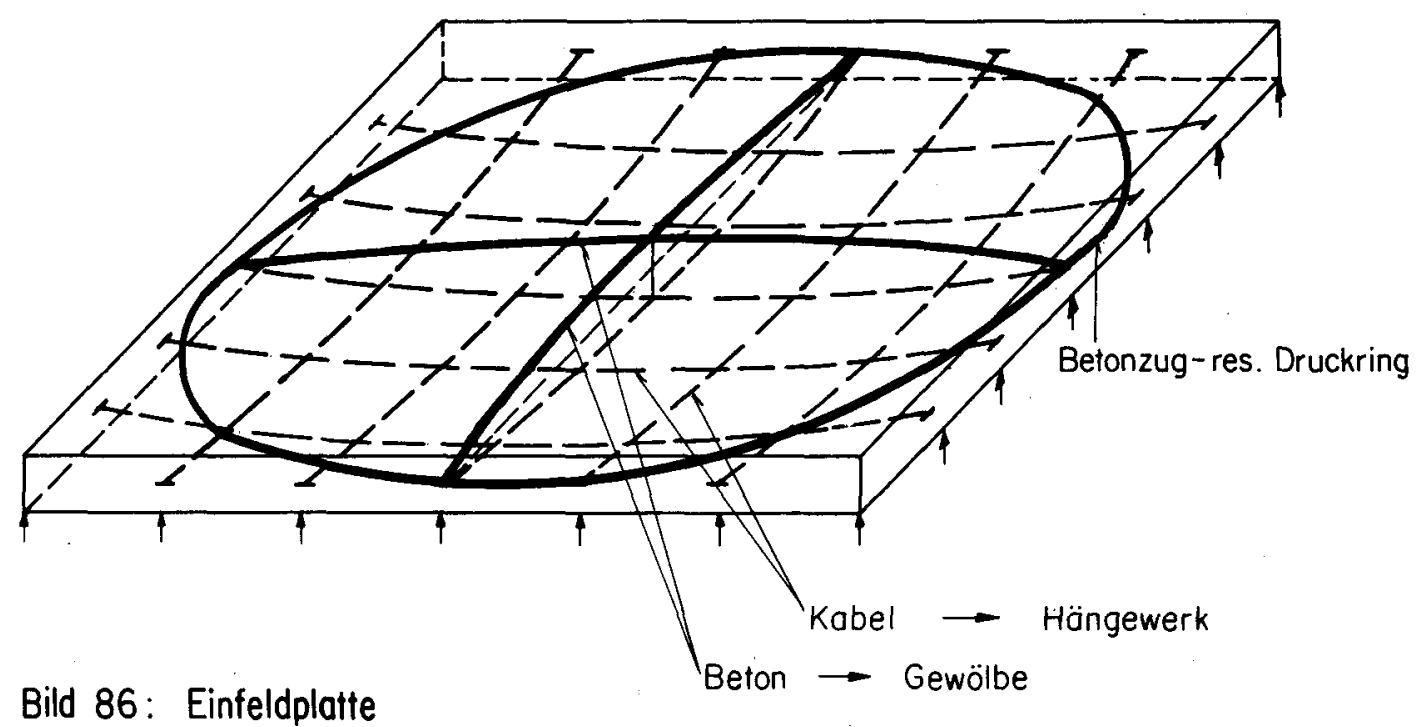


Ein aus dieser Platte herausgeschnitten gedachter Streifen wird schematisch in Bild 87 dargestellt. Der Beton wird als flacher Druckbogen resp. flache Schale, der Vorspannstahl als Hängewerk eingeführt. Die seitliche Feder soll den Zug- resp. Druckring der Einfeldplatte oder den horizontalen Widerstand anschliessender Felder oder Randträger simulieren. Die jeweiligen Randbedingungen sind durch Verträglich-* keitsbedingungen und entsprechende Verschiebungsansätze zu berücksichtigen. Der Einfluss einer schlaffen Armierung, auftretender Reibungskräfte und der Biegesteifigkeit des Betongewölbes wird durch einen Biegeträger berücksichtigt.

Balken mit verteilter Belastung parabolische Kabelführung

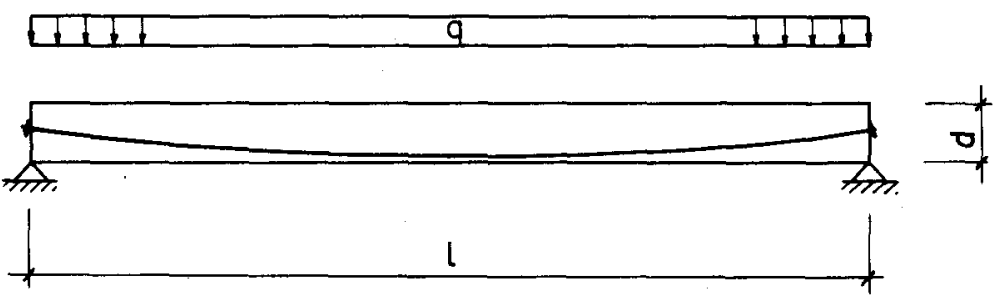

Modell

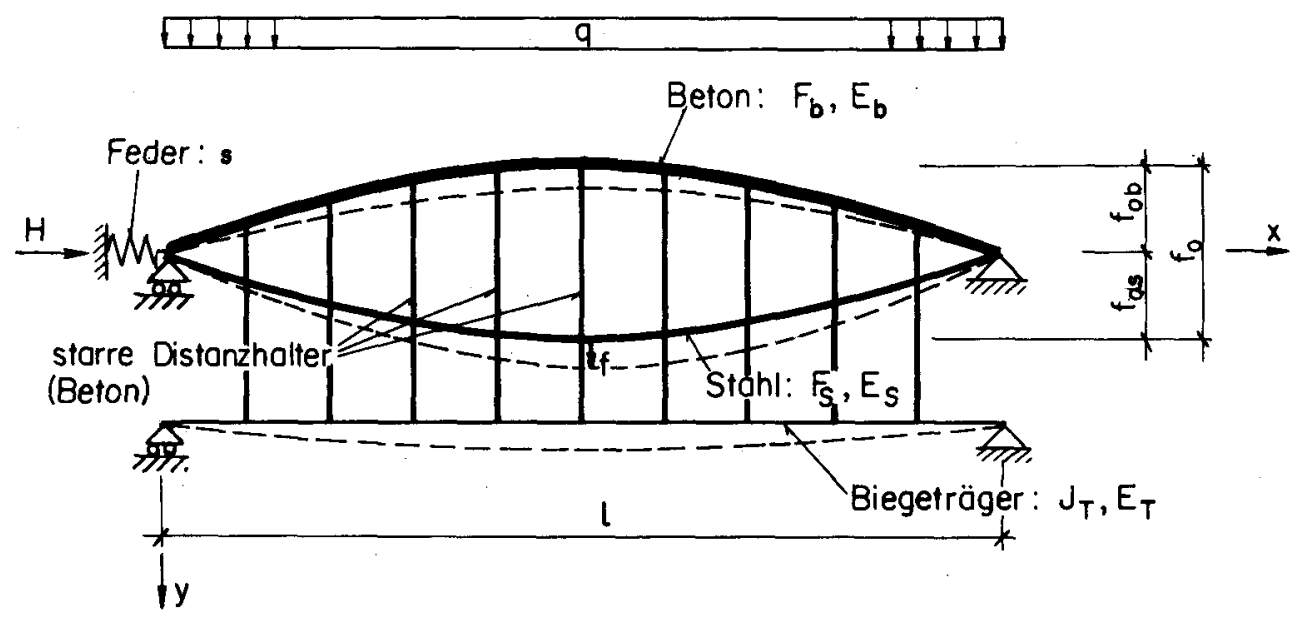

Bild 87 : Aus einer Platte herausgeschnitten gedachter Plattenstreifen 
Dies führt auf ein gekoppeltes Tragsystem, an dem die einzelnen Anteile an der Tragfähigkeit bestimmt werden können, wie dies in Bild 88 dargestellt ist. Dabei ist zu beachten, dass das Gleichgewicht am deformierten system formuliert werden muss (Theorie 2. Ordnung). Mit diesem Modell soll ein besserer Einblick in das Tragverhalten von vorgespannten Platten ohne Verbund gewonnen werden.

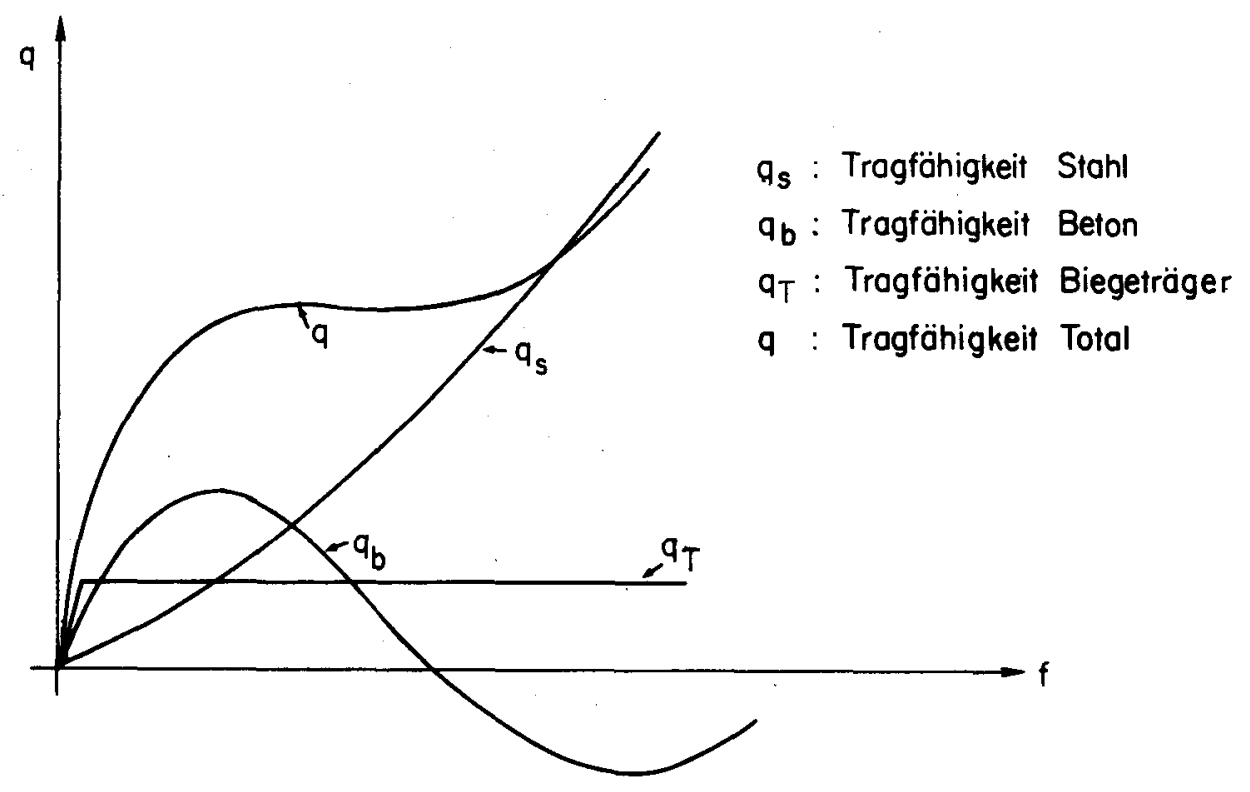

Bild 88: Qualitativer Verlauf der einzelnen Anteile an der Tragfähigkeit 
ANHANG B

BERECHNUNG DER THEORETISCHEN WERTE

I. PLATtE P 1

1. Grundwerte

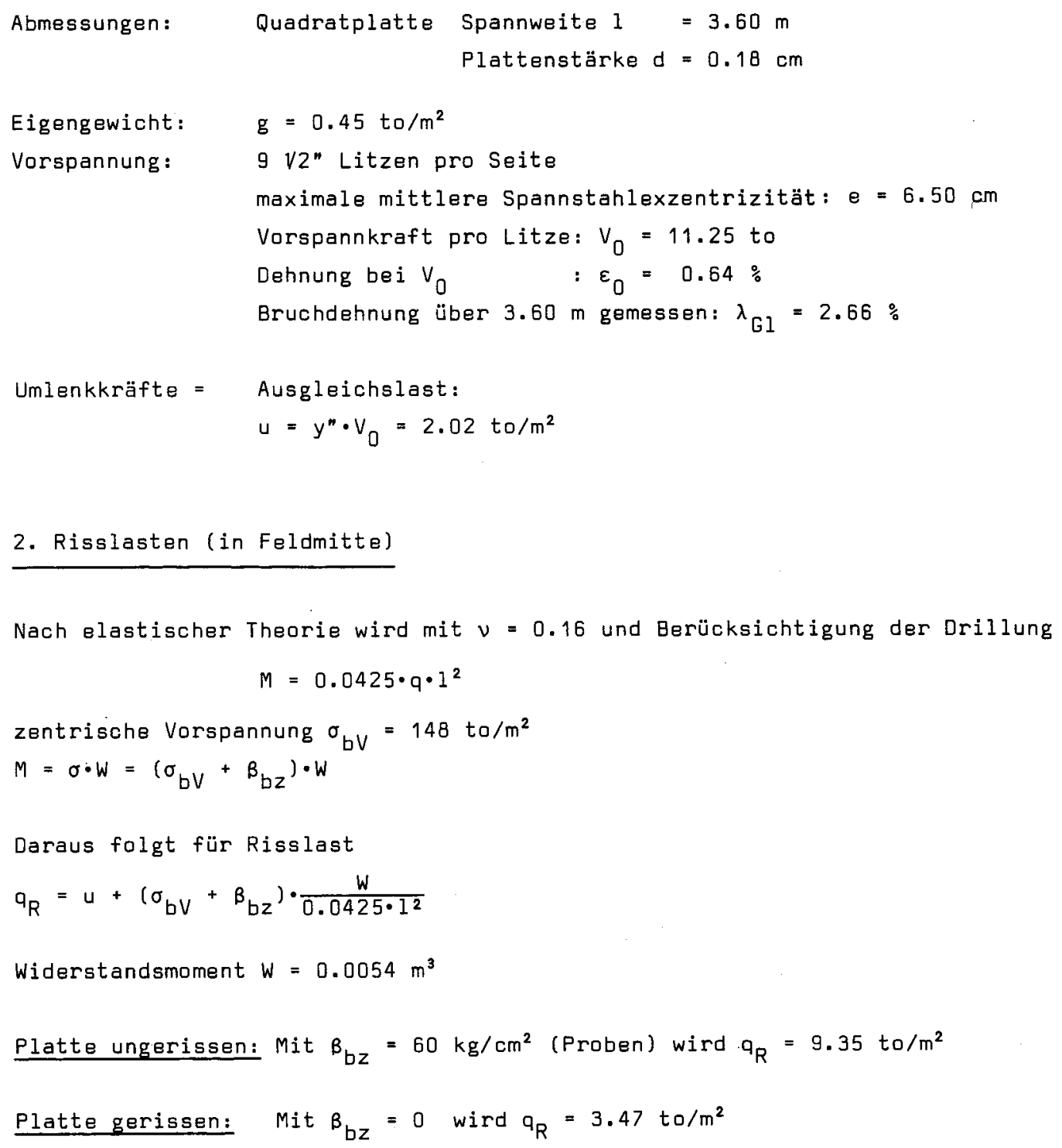

Für die Durchbiegungsfläche der Litzen wird folgender Näherungsansatz gemacht: 


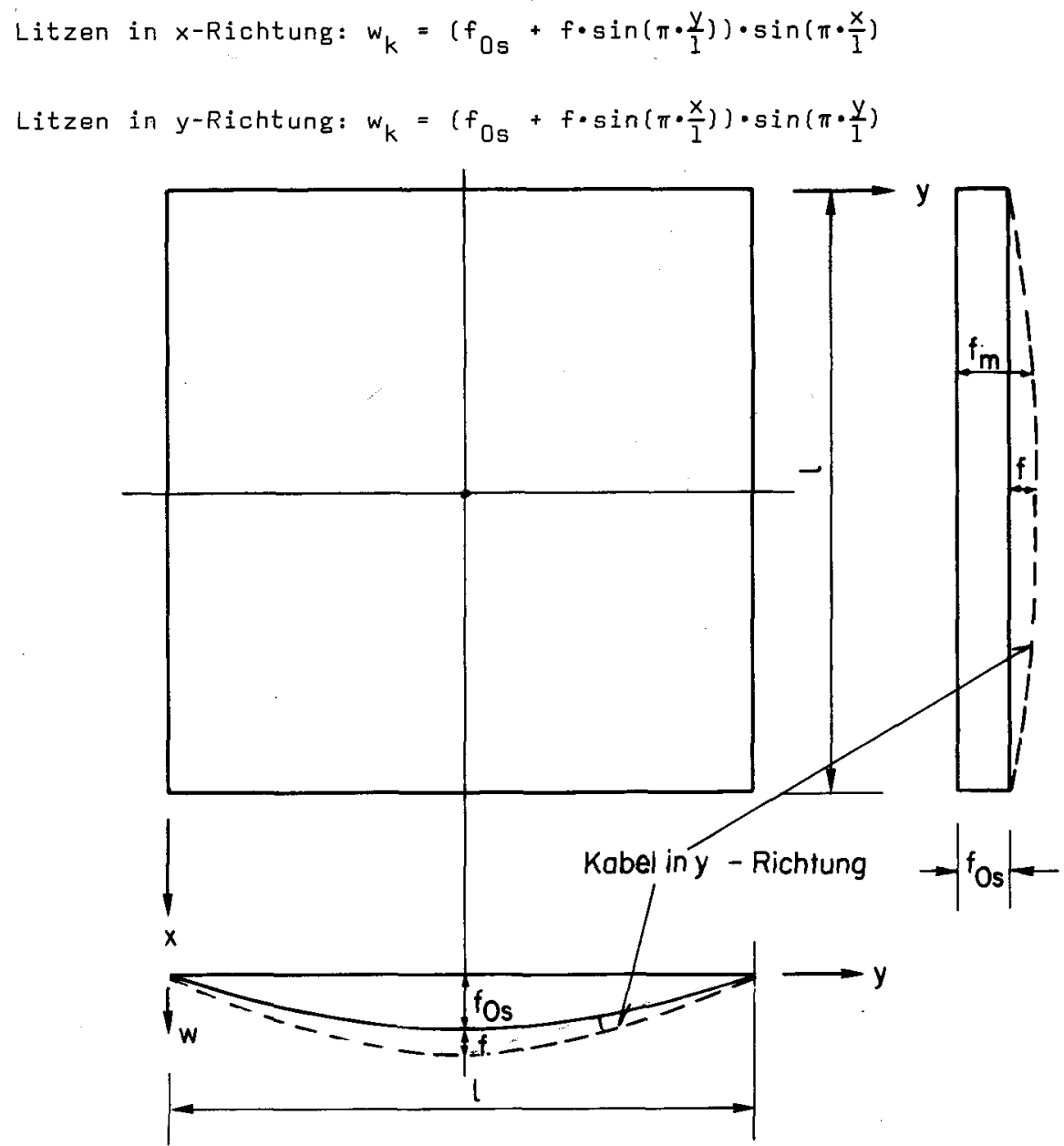

Bild 89: Durchbiegungsfläche der Litzen in Platte

Maximal mögliche Durchbiegung bei Litzenbruch:

Zusätzliche Dehnung $\Delta \varepsilon=\lambda_{G I}-\varepsilon_{0}=2.02 \%$

Kabeldehnung infolge Durchbiegung:

Für einen Sinus-Ansatz führt die Integration der Bogenlänge unter Vernachlässigung Glieder höherer Ordnung auf

$$
\varepsilon=\frac{\pi^{2}}{4} \cdot \frac{1}{1^{2}}\left[\left(f_{0 s}+f\right)^{2}-f_{0 s}^{2}\right]-\ldots+\ldots
$$

Daraus folgt die maximale Durchbiegung in Feldmitte

$$
\begin{aligned}
& f=\sqrt{\frac{4}{\pi^{2} \cdot I^{2} \cdot \varepsilon+f_{O S}^{2}}}-f_{O s} \\
& f=27 \mathrm{~cm}
\end{aligned}
$$

Tragfähigkeit der Kabel in x-Richtung bei maximaler Durchbiegung: Mittlere Durchbiegung $f_{m}=f_{O s}+\frac{2}{\pi} \cdot f$ 
Aus $M=q \cdot \frac{l^{2}}{g}=V \cdot f_{m} \cdot \frac{1}{1}$ folgt $q=V \cdot f m \cdot \frac{8}{\ell^{3}}$

$q=\frac{B \cdot V}{1^{3}}\left(f_{0 s}+\frac{2}{\pi} \cdot f\right)$

Mit $V_{\text {Bruch }}=16.77$ to pro Litze (statischer Bruchwert) wird aus beiden Richtungen $\underline{q_{u}}=\underline{11.90 \text { to } / \mathrm{m}^{2}}$

4. Bezugswerte

\section{a) Bruchlast unter der Annahme, dass Litzen in Verbund sind}

Oberer Grenzwert mit kombiniertem Linien-Fächer-Mechanismus, keine obere Armierung.

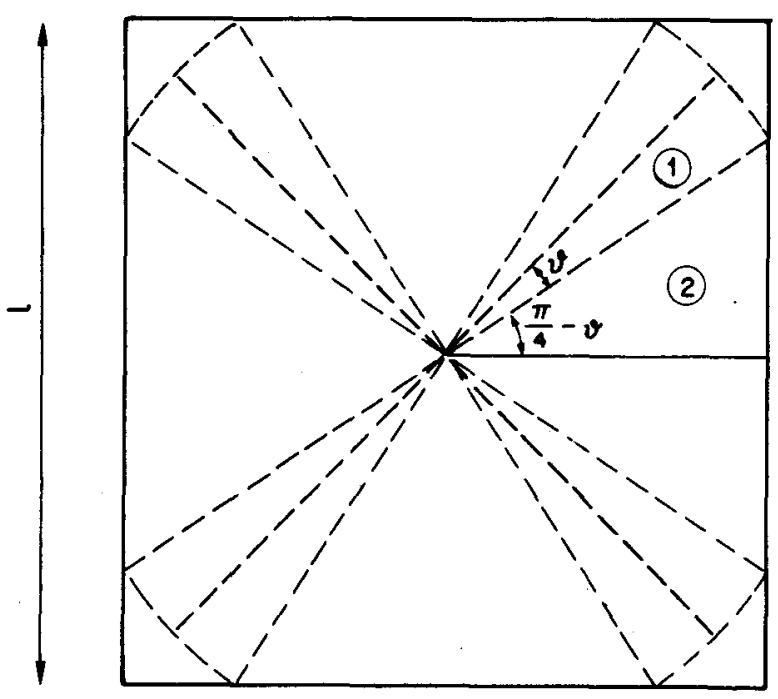

Bild 90: Bruchmechanismus

Berechnung des plastischen Momentes:

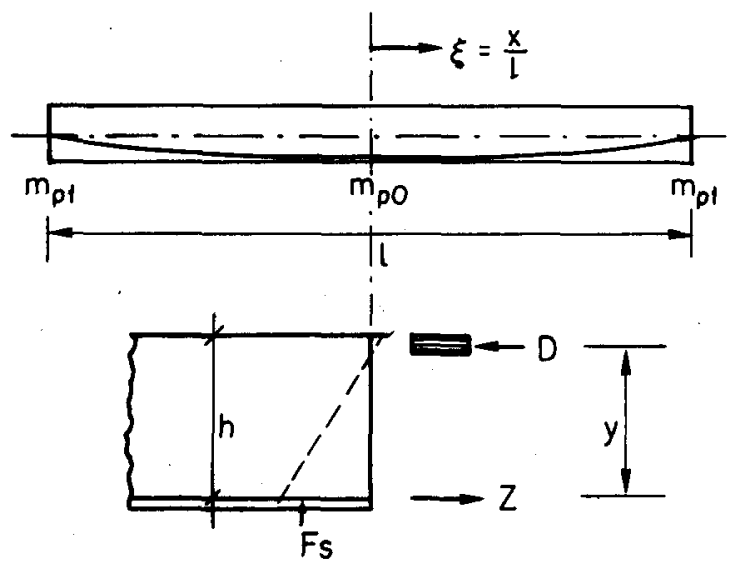

Bild 91: Plastische Momente 
Mit einer Prismendruckfestigkeit von $330 \mathrm{~kg} / \mathrm{cm}^{2}$ berechnet sich das maximale plastische Moment zu

$$
m_{p}=\left(h-\frac{Z}{2 \cdot \beta_{p} \cdot b}\right) \cdot z
$$

Feldmitte: $m_{p 0}=6.20 \mathrm{mt}$

Rand: $\quad m_{p 1}=3.50 \mathrm{mt}$

Ueber die ganze Spannweite wird somit

$$
m_{p}=m_{p 0}-4 \cdot\left(m_{p 0}-m_{p 1}\right) \cdot \xi^{2}
$$

Arbeit der äusseren Kräfte: $A_{a}=q \cdot \frac{1^{2}}{24}\left[\frac{\vartheta}{\cos ^{2}\left(\frac{\pi}{2}-\vartheta\right)}+\operatorname{tg}\left(\frac{\pi}{4}-\vartheta\right)\right]$

Arbeit der inneren kräfte: $A_{i}=m_{p 0}\left[\vartheta-\frac{\gamma}{4 B} \cdot \frac{(12 \cdot \vartheta-\sin 4 \vartheta)}{\cos ^{2}\left(\frac{\pi}{4}-\vartheta\right)}+\operatorname{tg}\left(\frac{\pi}{4}-\vartheta\right)-\frac{\gamma}{3} \cdot \operatorname{tg}^{3}\left(\frac{\pi}{4}-\vartheta\right)\right]$ wobei: $r=\frac{m_{p 0}-m_{p 1}}{m_{p 0}}$

Durch Variation von $\vartheta$ erhält man den kleinsten oberen Grenzwert bei $\vartheta=5$ bis $10^{\circ} \mathrm{zu}$

$$
\underline{q_{u v}}=9.60 \mathrm{to} / \mathrm{m}^{2}
$$

Mit der statischen Methode können mit Ansätzen für Gleichgewicht und Kontrolle der Plastizitätsbedingungen durch Handrechnung oder mit Programm IRIS [2] etwa gleich grosse untere Grenzwerte für die Traglast bestimmt werden.

Das heisst, dass $q_{u v}=9.60 \mathrm{to} / \mathrm{m}^{2}$ mit guter Genauigkeit der Traglast entspricht.

\section{b) Werte mit ACI-Recommendations (1974) [1]}

- Beschränkung der Betonspannungen auf $0.53 \cdot \sqrt{\beta_{p}}\left(\beta_{p}\right.$ in $\left.\left[\mathrm{kg} / \mathrm{cm}^{2}\right]\right)$. Mit $\beta_{p}=330 \mathrm{~kg} / \mathrm{cm}^{2}$ wird

$\sigma_{b z}=9.63 \mathrm{~kg} / \mathrm{cm}^{2}$

Daraus folgt $q_{z u l ~}^{A C I}=4.41 \mathrm{to} / \mathrm{m}^{2}$

- Bruch:

Stahlspannungen im Bruch:

$\sigma_{s}=\sigma_{s 0}+700 \mathrm{~kg} / \mathrm{cm}^{2}+\frac{B_{p}}{100 \cdot \mu_{s}}=15340 \mathrm{~kg} / \mathrm{cm}^{2}$

daraus wird die Bruchlast

$q_{u}^{A C I}=8.15$ to $/ \mathrm{m}^{2}$ und die zulässige Belastung

$\mathrm{Q}_{\mathrm{ZU1}}^{\mathrm{ACI} 2} 2=4.87 \mathrm{to} / \mathrm{m}^{2}$ 
Die massgebende zulässige Last folgt aus dem Kriterium der Beschränkung der Betonspannung $\mathrm{zu} \mathrm{q}_{\mathrm{zUl}}^{\mathrm{ACI}}=4.41$ to $/ \mathrm{m}^{2}$.

\section{PLATTENSTREIFEN PS 1 BIS PS 5}

1. Grundwerte

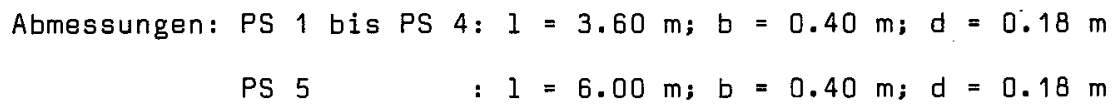

Schlaffe Armierung: Nur in PS 4. Box-Ultra mit einer Streckgrenze von

$\sigma_{\mathrm{e}} 0.2=5.2 \mathrm{to} / \mathrm{cm}^{2}$

Umlenkkräfte = Ausgleichslast

$$
\begin{aligned}
& u=y " \cdot V_{0} \\
& \text { PS } 1 \text { bis PS 4: } u=0.504 \text { to } / \mathrm{m} \\
& \text { PS } 5 \quad: u=0.18 \mathrm{to} / \mathrm{m}
\end{aligned}
$$$$
\operatorname{mil} y^{\prime \prime}=\frac{8 \cdot c}{e^{2}}
$$

\section{Risslasten}

$M=q \cdot \frac{1^{2}}{8}$

Zentrische Vorspannung: $\sigma_{b V}=156$ to $/ \mathrm{m}^{2} \quad$

Daraus folgt für die Risslast

$$
a_{R}=u+\left(\sigma_{b V}+\beta_{b z}\right) \cdot w \cdot \frac{\theta}{1^{2}}
$$

$W=0.00216 \mathrm{~m}^{3}$ 


\begin{tabular}{|c|c|c|c|}
\hline \multirow{2}{*}{} & \multirow{2}{*}{$\beta_{b z}$} & \multicolumn{2}{|c|}{$q_{R}$} \\
\cline { 2 - 4 } & & $\sigma_{b u}=\beta_{b z}$ & $\sigma_{b u}=0$ \\
\cline { 2 - 4 } & $\mathrm{kg} / \mathrm{cm}^{2}$ & $t_{0} / \mathrm{m}$ & $t_{0} / \mathrm{m}$ \\
\hline PS 1 & 43 & 1.29 & 0.71 \\
PS 2 & 64 & 1.57 & 0.71 \\
PS 3 & 58 & 1.49 & 0.71 \\
PS 4 & 57 & 1.47 & 0.71 \\
PS 5 & 48 & 0.49 & 0.25 \\
\hline
\end{tabular}

Tabelle 12: Risslasten Plattenstreifen

\section{Bruchlasten}

Annahmen: - Seitliche Haltung ist vorhanden

- Stahl trägt als Hängewerk

- Die Bruchdehnung der Litzen ist für alle PS gleich wie im Vorversuch $(2.66 \%)$.

Für den Durchbiegungsverlauf wird folgender Ansatz gemacht:
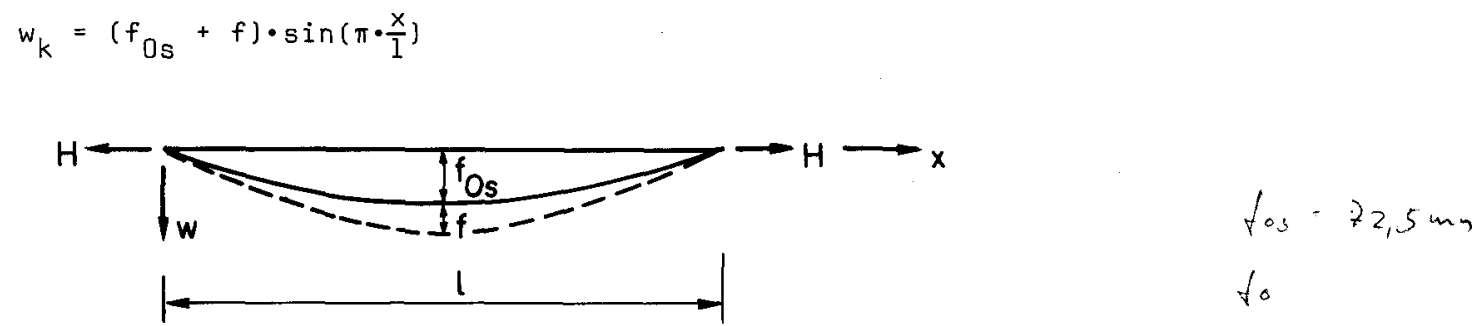

to

Bild 92 : Durchbiegungsverlauf der Litze in Plattenstreifen

Wie bei $P 1$ bestimmt sich die maximale Durchbiegung in der Mitte wie folgt:

$$
f=\sqrt{\frac{4}{\pi^{2}} \cdot 1^{2} \cdot \varepsilon+f_{0 s}^{2}}-f_{0 s}
$$

$$
\varepsilon_{4}=2,02 \%=20,2 \%
$$

PS 1 bis PS 4: $f=0.26 \mathrm{~m}$

PS 5

$: f=0.47 \mathrm{~m} V$ 
Aus $\quad q \cdot \frac{1^{2}}{8}=V \cdot\left(f_{0 s}+f\right)$

folgt $q=V \cdot\left(f_{0 s}+f\right) \cdot \frac{8}{1^{2}}$

PS 1, PS 2: $q_{u}=3.44 \mathrm{to} / \mathrm{m}$

PS 4: Biegeanteil schlaffe Armierung $F_{E}=1.51 \mathrm{~cm}^{2}$

$$
\begin{aligned}
& m_{p}=\left(h-\frac{F_{e} \cdot \sigma_{f}}{2 \cdot \beta_{p} \cdot b}\right) \cdot F_{e} \cdot \sigma_{f} \quad, \sigma_{f}=\sigma_{e} 0.2 \\
& q_{u}=4.19 \mathrm{to} / \mathrm{m}
\end{aligned}
$$

PS 5

$$
: q_{u}=2.02 \text { to } / \mathrm{m}
$$

\section{Bezugswerte}

4.1 Bruchlasten unter der Annahme, dass Litzen in Verbund sind

$$
\begin{array}{rlrl}
\text { Bruchmoment } m_{p} & =\left(h-\frac{F_{s} \cdot \beta_{z s}+F_{e} \cdot \sigma_{f}}{2 \cdot \beta_{p} \cdot b}\right) \cdot\left(F_{s} \cdot \beta_{z s}+F_{e} \cdot \sigma_{f}\right) & , \sigma_{f}=\sigma_{e} 0.2 \\
q_{u v}=m_{p} \cdot \frac{8}{1^{2}} \quad z=F_{s} \cdot \beta_{z s}+F_{e} \cdot \sigma_{f} & , \sigma_{f}=\sigma_{e} 0.2
\end{array}
$$

\begin{tabular}{|c|c|c|c|c|}
\hline & $B_{P}$ & $z$ & $m_{P}$ & $q_{u V}$ \\
\cline { 2 - 5 } & $\mathrm{kg} / \mathrm{cm}^{2}$ & $t_{0}$ & $\mathrm{mt}$ & $t_{0} / \mathrm{m}$ \\
\hline PS 1 & 342 & 16.77 & 2.62 & 1.62 \\
PS 2 & 359 & 16.77 & 2.63 & 1.62 \\
PS 3 & 327 & 16.77 & 2.62 & 1.62 \\
PS 4 & 281 & 24.62 & 3.73 & 2.30 \\
PS 5 & 390 & 16.77 & 2.63 & 0.58 \\
\hline
\end{tabular}

Tabelle 13: Bruchlasten unter der Annahme, dass Litzen in Verbund sind 


\subsection{Werte mit ACI-Recommendations (1974) [1]}

- Beschränkung der Betonzugspannungen

keine schlaffe Armierung: $\sigma_{b z}=0.53 \cdot \sqrt{\beta_{p}} \quad\left[\mathrm{~kg} / \mathrm{cm}^{2}\right]$

mit schlaffer Armierung : $\sigma_{b z}=1: 59 \cdot \sqrt{B_{p}}$

$q_{z u I}^{A C I}=u+\left(\sigma_{b V}+\sigma_{b z}\right) \cdot w \cdot \frac{8}{1^{2}}$

- Bruch

Stahlspannungen im Bruch: $\sigma_{s}=\sigma_{s 0}+700 \mathrm{~kg} / \mathrm{cm}^{2}+\frac{\beta p}{100 \cdot \mu_{s}}$

\begin{tabular}{|c|c|c|c|c|c|c|c|}
\hline & $\beta_{P}$ & $\sigma_{b z}$ & $q_{z U 1.1}^{A C I}$ & $\sigma_{s}$ & $q_{u}^{A C I}$ & $q_{z U I ~ 2}^{A C I}$ & $q_{z U 1}^{A C I}$ \\
\cline { 2 - 7 } & $\mathrm{kg} / \mathrm{cm}^{2}$ & $\mathrm{~kg} / \mathrm{cm}^{2}$ & $t o / \mathrm{m}$ & $t_{0} / \mathrm{cm}^{2}$ & $t_{0} / \mathrm{m}$ & $t_{0} / \mathrm{m}$ & to/m \\
\hline PS 1 & 342 & 9.8 & 0.84 & 15.4 & 1.40 & 0.85 & 0.84 \\
PS 2 & 359 & 10.0 & 0.85 & 15.6 & 1.40 & 0.85 & 0.85 \\
PS 3 & 327 & 9.6 & 0.84 & 15.3 & 1.38 & 0.84 & 0.84 \\
PS 4 & 281 & 26.7 & 1.07 & 15.0 & 2.06 & 1.23 & 1.07 \\
PS 5 & 390 & 10.5 & 0.31 & 15.8 & 0.52 & 0.34 & 0.31 \\
\hline
\end{tabular}

Tabelle 14: Vergleichswerte nach ACI-Empfehiungen (1974) 